\title{
Studies on Resistance of Oilseed Rape (Brassica napus) to Verticillium longisporum - Interaction with Drought Stress, Role of Xylem Sap Modulations and Phenotyping Under Controlled and Field Conditions
}

\author{
Dissertation \\ to obtain the Ph.D. degree \\ in the international Ph.D. Program for Agricultural Sciences in Göttingen (IPAG) \\ at the Faculty of Agricultural Sciences, \\ Georg-August-University Göttingen, Germany
}

Daniel Teshome Lopisso

Born in Addis Ababa, Ethiopia 


\section{D7}

1. Name of referee: Prof. Dr. Andreas von Tiedemann

2. Name of co-referee: Prof. Dr. Petr Karlovsky

Date of disputation: 19 November 2014 


\section{Table of contents}

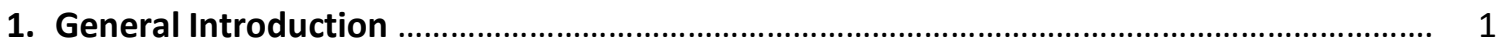

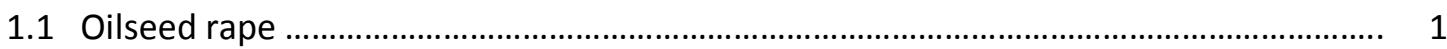

1.2 Verticillium diseases ...............................................................................................

$1.3 \mathrm{~V}$. longisporum: the youngest vascular pathogen of oilseed rape ................................ 3

1.3.1 Significance and geographical distribution of $V$. longisporum ......................... 5

1.3.2 V. longisporum disease cycle ........................................................................ 6

1.3.3 Pathogenicity factors in V. longisporum ........................................................ 8

1.3.4 Management of $V$. longisporum in oilseed rape ................................................. 8

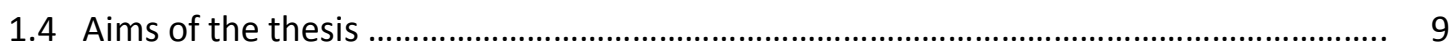

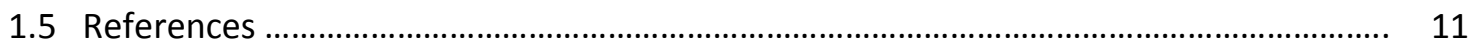

2. Phenotypic and molecular evaluation of Brassica napus lines for $V$. longisporum resistance under greenhouse, outdoor and field conditions ...................................... 17

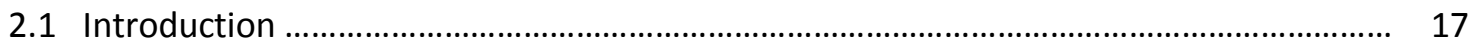

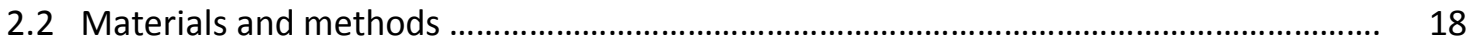

2.2.1 Greenhouse screening .............................................................................. 18

2.2.1.1 Plant material ............................................................................... 18

2.2.1.2 Experimental design .......................................................................... 19

2.2.1.3 Inoculation procedures and disease scoring ........................................... 19

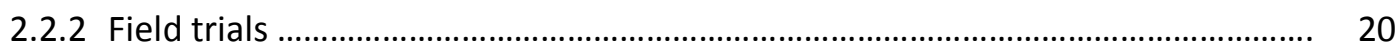

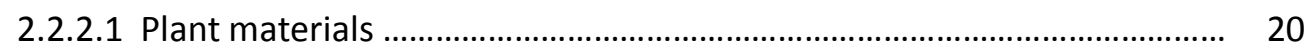

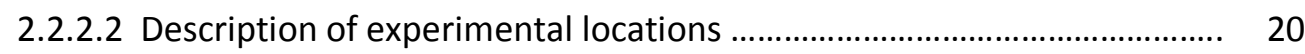

2.2.2.3 Experimental design ............................................................................. 20

2.2.2.4 Inoculation and agronomic practices ................................................ 21

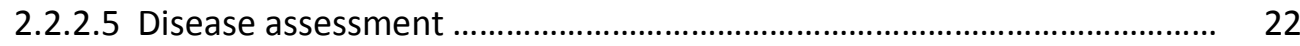

2.2.3 Outdoor experiment .................................................................................. 23

2.2.3.1 Plant materials and growing conditions ............................................. 23

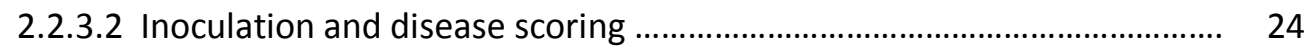

2.2.3.3 Experimental design and outdoor environmental conditions ................ 24

2.2.3.4 Agronomic and disease data collection .............................................. 25

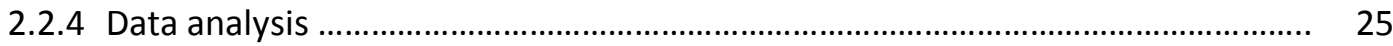

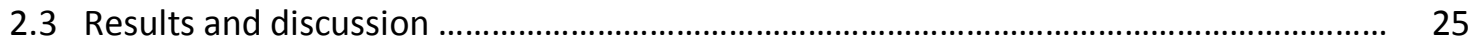

2.3.1 Greenhouse screening ........................................................................... 25

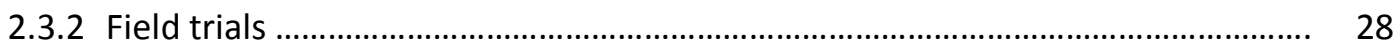

2.3.2.1 B. napus $\mathrm{DH}$ lines evaluated for $V$. longisporum resistance in multi- 
location field trials for three consecutive seasons.

2.3.2.2 Different sets of $B$. napus lines screened for $V$. longisporum resistance under field conditions for one season.

2.3.2.3 Determination of the critical crop growth stage for molecular detection and differential quantification of $V$. longisporum disease severity in field grown winter oilseed rape plants

2.3.3 Outdoor experiment 48

2.4 References 52

\section{Searching for cultivar-related resistance factors to $V$. longisporum in oilseed rape}

(Brassica napus) xylem sap 54

3.1 Introduction 54

3.2 Materials and methods 56

3.2.1 Treatments and experimental design ........................................................ 56

3.2.2 Plant material and cultivation ..................................................................... 56

3.2.3 Fungal growth and inoculation technique ................................................... 57

3.2.4 Xylem sap collection ............................................................................... 58

3.2.5 Disease evaluation ................................................................................... 58

3.2.6 In vitro bioassay ....................................................................................... 59

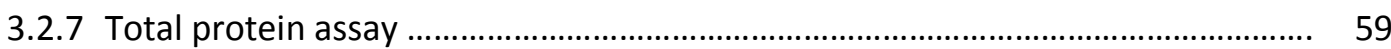

3.2.8 Quantification of xylem sap total carbohydrate content .................................. 59

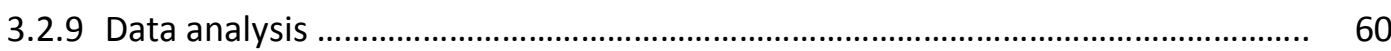

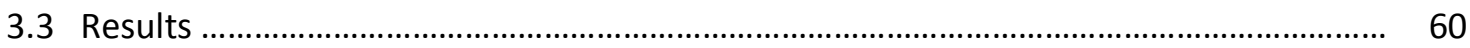

3.3.1 Greenhouse experiments ............................................................................ 60

3.3.2 Analysis of $V$. longisporum growth on B. napus xylem sap ............................... 62

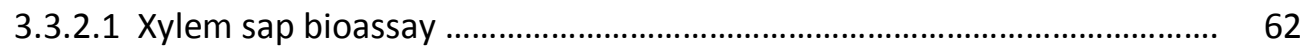

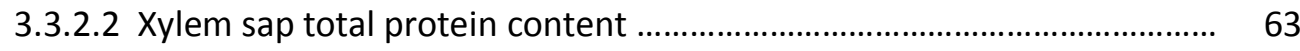

3.3.3 Analysis of $V$. longisporum growth in xylem sap extracted from different age plants 65

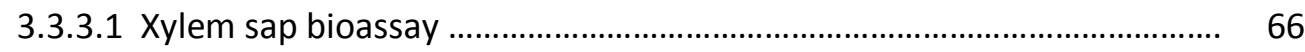

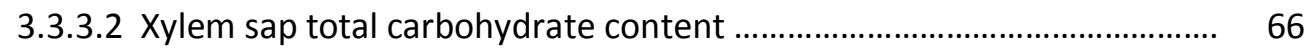

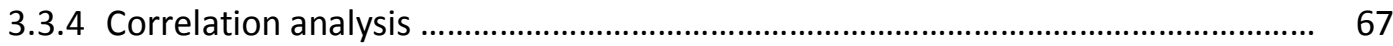

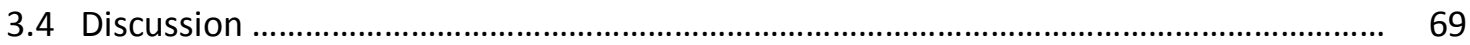

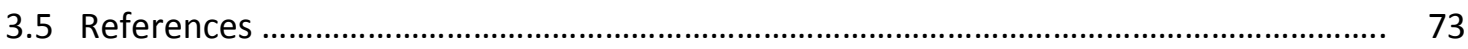


4. Physiology, gene expression and agronomic performance of winter oilseed rape under drought stress and infection with $V$. longisporum 77

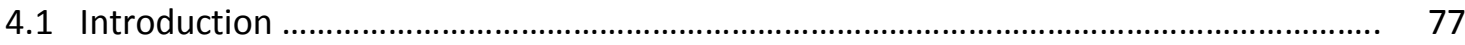

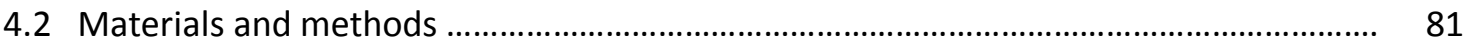

4.2.1 Treatments and experimental conditions ...................................................... 81

4.2.2 Determination of field capacity in the soil substrate ..................................... 83

4.2.3 Plant material and growing conditions ..........................................................

4.2.4 Fungal isolate and inoculation procedures ..................................................... 84

4.2.5 Determination of soil water content and maintenance of watering regime ...... 84

4.2.6 Plant sample collection and preservation ...................................................... 85

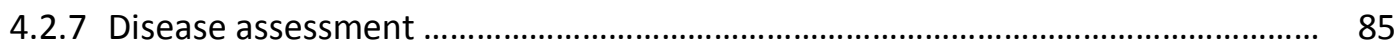

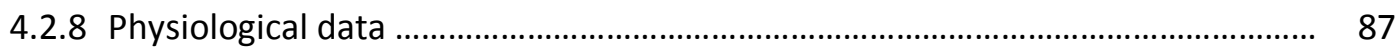

4.2.9 Measurement of yield and agronomic variables ............................................ 89

4.2.10 Expression of drought responsive genes ..................................................... 89

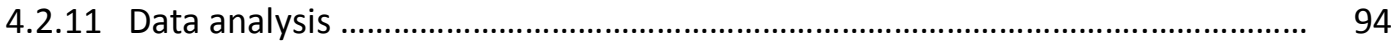

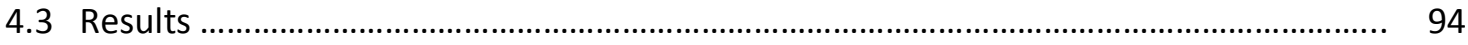

4.3.1 V. longisporum disease development under drought stress conditions ............ 94

4.3.2 Physiological responses to $V$. longisporum infection and drought stress ........... 98

4.3.3 Effect of drought stress and V. longisporum infection on expression of drought responsive genes ............................................................................ 104

4.3.4 Effect of $V$. longisporum and drought stress on yield and morphological traits.. 107

4.3.5 Correlation and regression analyses ........................................................... 116

4.3.6 Principal component analysis ...................................................................... 120

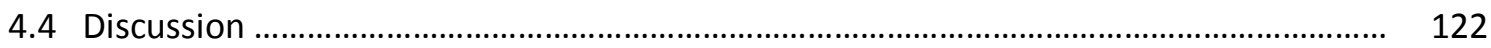

4.4.1 B. napus response to $V$. longisporum under drought stress .............................. 122

4.4.2 B. napus physiology during drought stress and infection with V. longisporum .. 125

4.4.3 Expression of drought responsive genes during drought stress and infection with V. longisporum

4.4.4 Single and combined effects of $V$. longisporum infection and drought stress on yield and agronomic traits

4.4.5 Correlation, regression and principal component analyses .............................. 138

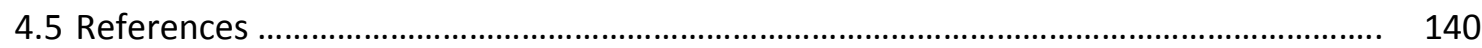

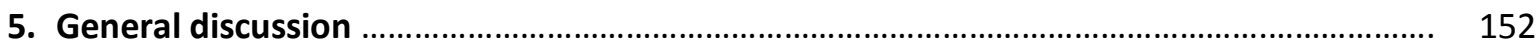

5.1 Identification of $B$. napus genotypes with enhanced resistance to $V$. longisporum..... 152

5.2 Searching for VL resistance factors in OSR xylem sap ............................................ 159 
5.3 B. napus - V. longisporum interaction under drought stress conditions .................... 162

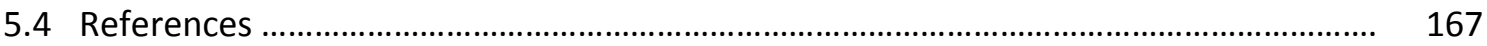

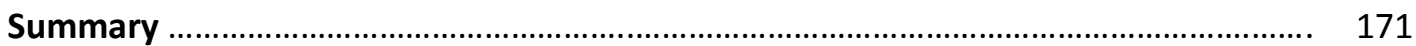

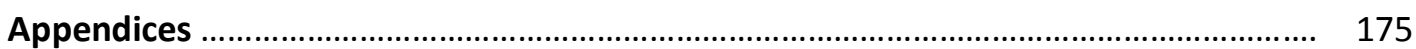

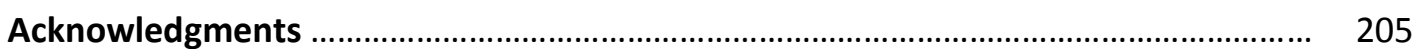

Selected contributions in non-reviewed journal paper/conference proceedings ...... 208

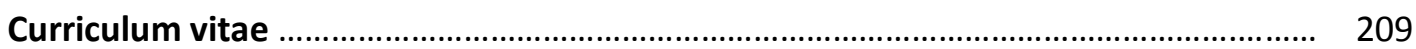

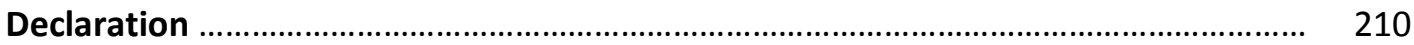




\section{General Introduction}

\subsection{Oilseed rape (Brassica napus L.)}

Oilseed rape (OSR) is one of the economically most important crops in the family Brassicaceae which consists of approximately 338 genera and 3709 plant species (Warwick and Al-Shehbaz, 2006). It is an allotetraploid species (AACC genome; $2 n=38$ ) derived from a spontaneous hybridization between turnip rape (Brassica rapa L.; $2 n=20 ; A A$ genome) and cabbage (Brassica oleracea L.; $2 n=18$; CC genome). B. napus comprises two subspecies, napobrassica and napus. The latter includes the most widely cultivated winter and spring rapeseed forms (Song and Osborn, 1962; Snowdon et al., 2007). The centre of origin for the two diploid parents of $B$. napus is located around the Mediterranean basin (B. oleracea) and Euro-Siberia and the Mediterranean basin (B. rapa) (Warwick et al., 2009). Regarding $B$. napus however, the exact period and place of origin is not known but its domestication is believed to have occurred in Europe in the early Middle Ages and later commercial plantings primarily for its use as oil for lamps were recorded in the Netherlands as early as the $16^{\text {th }}$ century (OECD, 1997).

OSR also called rapeseed, swede rape or canola (Orlovius, 2013) is a winter or spring type annual plant. The spring types differ from winter varieties because they do not require vernalization (winter chilling) to flower, although vernalization speeds up flowering. The name 'canola' refers to the high quality varieties with significantly lower contents of two naturally occurring harmful metabolites, erucic acid (below $2 \%$ in oil) and glucosinolates (total glucosinolates of $30 \mu \mathrm{moles} / \mathrm{g}$ toasted oil free meal) (OGTR, 2012). The OSR plant, depending on the variety and environmental conditions, can grow up to $1.5 \mathrm{~m}$ high. Its stem is well branched and it has two types of roots, a deep tap root and fibrous lateral roots near the surface. Leaves are smooth and dark bluish green with few scattered hairs near the margin. Although wind or insect-borne cross-pollination is possible, OSR is generally considered as a self-fertile species. Its flowers are bright yellow with four petals and borne in clusters in the form of elongate racemes. Seeds are round, small (1.8-2.8 $\mathrm{mm}$ in diameter) and brown-black coloured (OECD, 1997; Sattell et al., 1998; Alford, et al., 2003; Orlovius, 2013).

OSR provides diverse economic and ecological benefits. The oil extracted from its seed is primarily used in human nutrition. The oil is also used as a raw material in non-food products such as in biodiesel and oleochemical industries. A residue from oil production, the cake, contains proteins and is used in animal nutrition. Integration of OSR in different cropping systems also provides several advantages since it has rapid growth characteristics in fall, it is grown as catch crop to capture part of the available soil nitrogen, which otherwise might be lost due to leaching during winter. OSR also provides excellent erosion control and good ground cover over winter. It is known as a 'break crop' for it is beneficial for the following crop in crop rotation. The characteristic large biomass production 
helps to suppress weed growth. Plant residues used as 'bio-fumigant' can significantly reduce weed and disease infestation in the following season. Furthermore, its deep tap root system can help in loosen plough pans and improve soil structure. Last but not least, OSR flowers serve as a source of nectar and pollen for honey bees (Sattell et al., 1998; Orlovius, 2003; Haramoto and Gallandt, 2004; Haramoto and Gallandt, 2005).

World OSR production is increasing rapidly. Since 2000, global OSR production has surpassed cottonseed and became the world's second most important source of vegetable oil after soybean (Carré and Pouzet, 2014). In 2012, it was produced on more than 34 million hectares with an estimated total production of 65 million tonnes (FAOSTAT, 2014). At present, Canada, China, EU, India, Australia, and Ukraine are the world's top major production areas (Figure 1.1). In particular, due to the expansion of the biodiesel industry, OSR has become the major oilseed in European agriculture and its production is increasing from time to time (Carré and Pouzet, 2014).

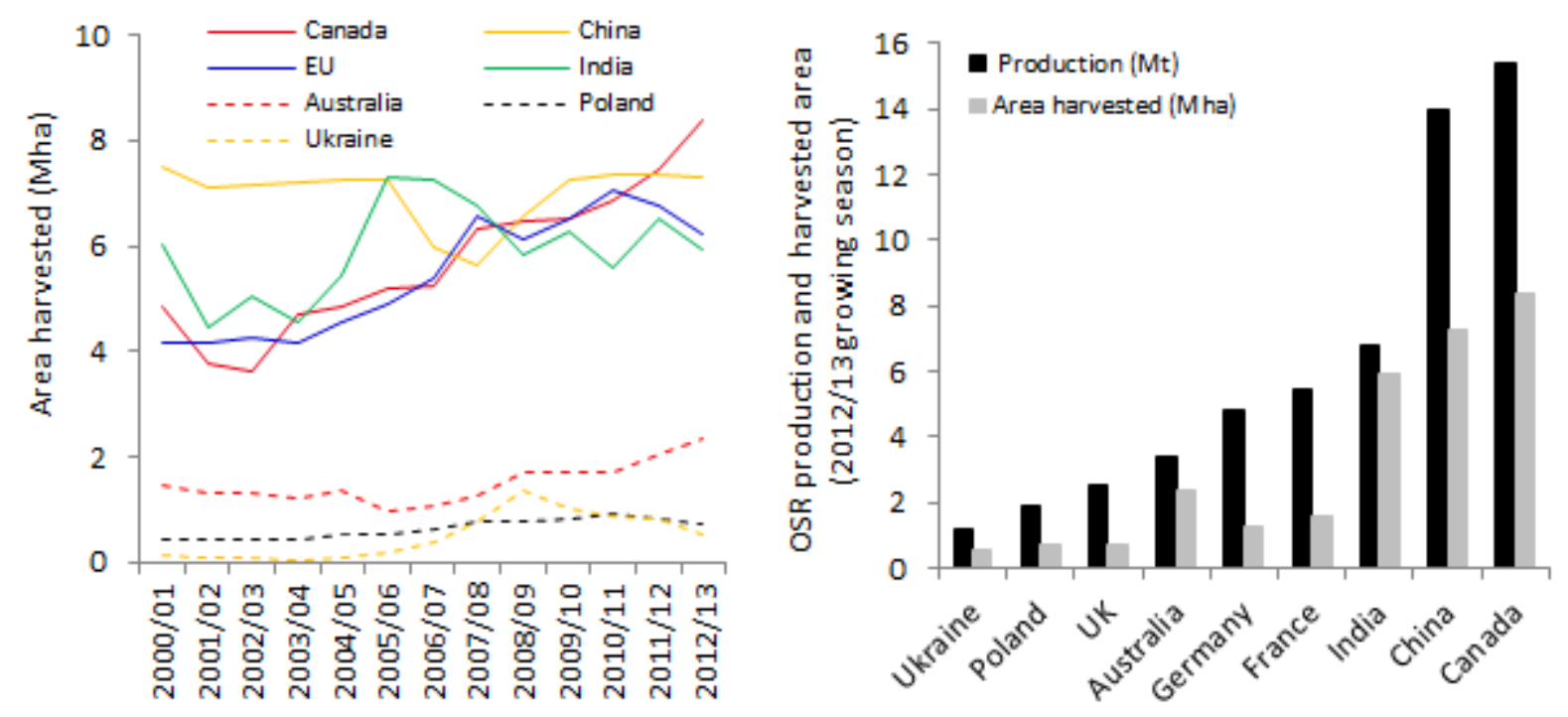

Figure 1.1 Proportion of OSR harvested area in major production regions of the world from 2000 to 2012 (left) and total rapeseed production in the nine top producing countries in 2012/13 growing season (right). Mha, million hectares. Mt, million tonnes. (Source: FAOSTAT, 2014).

The ever-increasing global OSR production is not without problems. Even though the degree of importance of the various production constraints varies depending on agro-ecological conditions, a number of biotic and abiotic stress factors can potentially affect growth and productivity of OSR. Cold stress is one of the primary abiotic stress factors. It causes a reduced number of seeds per plant and production of seeds with lightweight (Lardon and Triboi-Blondel, 1995). Similar effects, which ultimately lead to significant yield losses, can be induced by waterlogging occurring particularly at seedling and inflorescence emergence stages (Zhou and Lin, 1995). High temperature causes flower sterility and production of abnormal seeds (Polowick and Sawhney, 1988). On the other hand, water deficit stress interferes with plant development and several physiological processes that affect 
quantity and quality of yield (Sadaqat et al., 2003; Sinaki et al., 2007; Din et al, 2011). Among the biotic factors, a wide range of invertebrate and vertebrate pests (Lane and Gladders, 2000; Alford, et al., 2003) and different types of foliar and root pathogens (Lane and Gladders, 2000; Gabor et al., 2013; Leino, 2006) can cause yield reduction in OSR and its relatives. In addition, the presence of weeds in OSR fields not only competes for shared resources and causes yield reduction, but also interferes with harvesting and may contaminate grain yield (Gianessi et al., 2003). From the biotic factors, the fungal diseases caused by Leptosphaeria maculans, anamorph: Phoma ligam (Blackleg), Sclerotinia sclerotiorum (Sclerotinia stem rot), Alternaria brassicae (Alternaria black spot), Verticillium longisporum, Pyrenopeziza brassicae, anamorph: Cylindrosporium concentricum (Light leaf spot); the oomycete pathogens Albugo candida (White rust) and Peronospora parasitica (Downy mildew) and the protist pathogen Plasmodiophora brassicae (Clubroot) are among the economically most important production constraints in many OSR growing regions of the world (Gómez-Campo, 1999; Lane and Gladders, 2000; Alford et al., 2003).

\subsection{Verticillium diseases}

Verticillium is a small genus consisting of Ascomycete phytopathogens that cause vascular wilt diseases in several economically important vegetable, ornamental, forest and industrial crop species (Agrios, 2005). Recently, morphological, DNA sequence and herbarium based phylogenetic studies recognized ten species belonging to this genus (Verticillium sensu stricto). According to this study, the genus Verticillium fell into two clades, Flavexudans (with yellow pigmented hyphae) and Flavnonexudans (without yellow pigmented haphae). $V$. longisporum is placed under the clade Flavnonexudans, which also consists of $V$. alfalfae, $V$. dahliae, $V$. nonalfalfae, $V$. nubilum and its two unknown ancestral species (species A1 and species D1). The remaining five species ( $V$. albo-atrum, $V$. tricorpus, V. zaregamsianum, V. isaacii and V. klebahnii) belong to the clade Flavexudans (Inderbitzin et al., 2011b).

\section{$1.3 \mathrm{~V}$. longisporum: the youngest vascular pathogen of OSR}

The existence of a distinct physiological strain of $V$. dahliae with restricted host range to the cruciferous plant Brussels sprout (Brassica oleracea) was first reported from UK in 1957 (Isaac, 1957). Later in 1961, V. longisporum (VL) as a long-spored isolate of $V$. dahliae was reported from horseradish in Germany. At that time, it was described as a variety of $V$. dahliae and named $V$. dahliae var. longisporum (Stark, 1961). A few years later, auxotrophic mutant analysis of the longand short-spored isolates of $V$. dahliae demonstrated VL as a naturally occurring diploid species which is stable under laboratory conditions (Ingram, 1968). The first reliable molecular evidence for the presence of genetic variability in V. dahliae was shown by Messner et al. (1996), who based on RAPD-PCR and 18SrRNA sequencing analysis showed the separate clustering of $B$. napus derived 
isolates from those collected from a wide range of other hosts. A year later, a more extensive study that involved several techniques (morphological characters, enzymatic activity, DNA profiles and pathogenicity testing) and consisted of several crucifer isolates from different geographical regions showed VL to be a near-diploid species whose host rage is restricted to crucifers. This study proposed the taxonomic position of VL to be raised from a variety level to a higher, namely species rank (Karapapa et al., 1997).

Since then, several studies focusing on the taxonomy and phylogenetic relationship of VL have been conducted. Fahleson et al. (2004), based on nuclear and mitochondrial sequence analysis, have shown distinct features of $\mathrm{VL}$ and its close relation to $V$. albo-atrum and suggested $V L$ to be regarded as a separate species. In support of this study, Pantou et al. (2005) by molecular and immunochemical analysis of different $V L$ isolates reinforced recognition of $V L$ as a separate species and argued that VL is closer to V. albo-atrum than to V. dahliae. Clewes et al. (2008) showed the presence of two or more $\beta$-tubulin genes and $5 S$ rRNA associated sequences in VL but only one in most $V$. dahliae isolates and suggested the origin of $V L$ as an interspecific hybridization between two or three parental species similar to $V$. dahliae. Recent genome sequencing of two Swedish VL isolates showed $86 \%$ similarity with the $V$. dahliae genome. These isolates have double the genome size (approximately $70 \mathrm{Mb}$ ) than that of $V$. dahliae and $V$. albo-atrum and harbour more than 20,000 protein coding genes (Roos, 2014). Several other studies have also shown morphological, physiological and DNA based evidences (Table 1.1) that explain the distinct differences between VL and the closely related species V. dahliae (Karapapa et al., 1997; Zeise and Tiedemann, 2001; Steventon et al., 2002; Inderbitzin et al., 2011b). In addition, it is obvious that VL, unlike other Verticillium species including VD, does not induce wilting and its host range is restricted mainly to crucifers (Karapapa et al., 1997; Bhat and Subbarao, 1999; Zeise and Tiedemann, 2002). Quite recently, an extensive phylogenetic analysis based on ITS and five protein coding genes sequence data from over 200 Verticillium isolates, which represent a wide range of hosts and geographic regions, also confirmed that VL is a diploid hybrid. This study identified four different alleles in VL, each representing four different lineages (lineage A1, D1, D2 and D3). Accordingly, it was demonstrated that the origin of VL involved three different parental species $(V$. dahliae and two other unknown parental species called species A1 and species D1). Species A1 was involved in each hybridization events and resulted in three VL lineages, VL lineage A1/D1, VL lineage A1/D2 and VL lineage $A 1 / D 3 . V L$ lineage $A 1 / D 1$ originated from hybridization events between the two unknown species A1 and D1. This lineage is known to be confined in Europe and is pathogenic to OSR and cauliflower. Lineage A1/D2 is the product of hybridization between species $A 1$ and the other VD lineage D2. This lineage is confined to horseradish in Illinois (USA) and it is not known whether it causes disease in OSR. The third VL lineage, A1/D3, evolved from the hybridization of species A1 and 
the VD lineage D3, is avirulent on OSR and it is confined in Europe and Japan (Inderbitzin et al., 2011a).

Table 1.1 Evidences for a separate taxonomic grouping of $V$. longisporum from $V$. dahliae.

\begin{tabular}{lll}
\hline Parameters & V.dahliae & V. longisporum \\
\hline MS Shape $^{1,3,7}$ & mostly rounded or spherical & mainly elongate \\
Conidia size $^{1,5,7}$ & Short $(3.5-5.5 \mu \mathrm{m})$ & Long $(7.1-8.8 \mu \mathrm{m})$ \\
DNA content & Low (most isolates) & High (most isolates) \\
PPO activity & Strong (most isolates) & None (most isolate) \\
Culture filtrate fluorescence $^{1,3}$ & Absent & Present \\
Host range $^{1,2,4}$ & Broad (Vegetables, trees, legumes, & Mainly restricted to \\
Wilting symptom $^{6}$ & and ornamental crops) & Crucifers \\
\hline
\end{tabular}

MS, microsclerotia. PPO, extracellular polyphenol oxidase. 'Karapapa et al., 1997; ${ }^{2}$ Bhat and Subbarao, 1999; ${ }^{3}$ Zeise and Tiedemann, 2001; ${ }^{4}$ Zeise and Tiedemann, 2002; ${ }^{5}$ Steventon et al., 2002; ${ }^{6}$ Dunker et al., 2008.

${ }^{7}$ Inderbitzin et al., 2011b.

According to the evidences described in the above paragraphs, the present taxonomic position of VL as a distinct independent species in the Verticillium genus seems not controversial any more. As mentioned earlier, a new classification of the genus with five new species ( $V$. alfalfae, $V$. isaacii, $V$. klebahnii, V. nonalfalfae and V. zaregamsianum) has been recently demonstrated (Inderbitzin et al., 2011b). Molecular methods that discriminate not only among the different Verticillium species, but also among VL lineages or between VL isolates that differ in virulence towards $B$. napus are available (Tran et al., 2013; Inderbitzin et al., 2013). Nevertheless, the debate regarding the species nomenclature of VL (longisporum) which refers to the possession of larger-sized asexual spores seems not yet resolved. For instance, based on the reports in Zeise and Tiedemann (2001), Collins et al. (2003) and Inderbitzin et al. (2011b) who observed rare production of medium to long sized conidia by few isolates of the haploid species $V$. albo-atrum and $V$. dahliae, there is an argument saying that the name longisporum is confusing (Fahleson et al., 2004; Pantou et al., 2005; Inderbitzin et al., 2011b). Another somehow controversial issue, which might be related to the different VL lineages or correct identification of Verticillium species, is the host specificity of VL. Johansson et al. (2006) reported possible infections of highly susceptible plants outside the Brassicaceae. This contradicts with reports of earlier studies that showed Brassicaceae to be the restricted host range of this pathogen (Karapapa et al., 1997; Zeise and Tiedemann, 2002).

\subsubsection{Significance and geographical distribution of $V$. longisporum}

Although evidences showed that recent evolution of $V L$, the precise geographic location where this pathogen first originated is unknown. Based on current distribution of the different VL lineages, Inderbitzin et al. (2011a) speculated one of the following three regions for the origin of VL. These are Illinois (where VL lineage A1/D2 is restricted), Europe (where VL lineage A1/D1 and A1/D3 are 
confined) and Japan (where lineage A1/D3 is confined). Besides, analysis of the genetic variation among large collections of $V$. longisporum isolates showed significant geographic structuring of German, Swedish and Californian isolates, possibly due to long distance geographic separation of these locations by forest and water barriers (Fahleson et al., 2003). VL is currently known from Belgium, Czech Republic, France, Germany, Japan, the Netherlands, Poland, Southern Russia, Sweden, UK and USA (CA, IL) (CABI and EPPO, 2011). More importantly, field yield loss assessment studies in Germany (Dunker et al., 2008) and UK (Gladders, 2009) indicated significant yield loss potentials of this pathogen in OSR.

\subsubsection{V. longisporum disease cycle}

The life cycle of VL can be divided into three major phases: dormant, parasitic and saprophytic. During the dormancy period, the fungus survives in the soil or in plant debris via its microsclerotia (Fig. 1.3; Fig. 1.2). Microsclerotia are the major structures that enable the fungus to resist extreme environmental conditions and serve as a viable source of inoculum for several years. Contaminated or perhaps infected seeds can also serve as a source of inoculum. The parasitic phase starts when microsclerotia germinate and produce hyphae possibly in response to stimulation by root exudates (Leino, 2006; Berlanger and Powelson, 2000). Initial infection occurs during autumn primarily by direct penetration of epidermal cells of lateral roots and root hairs. Once the fungus has entered into the root cortex, it starts colonizing the root xylem vessels (Eynck et al., 2007) and spends most part of its life cycle in this host tissue. Systemic spread to the shoot is rather slow and infection can be latent up to nine months. VL infection in OSR induces plugging of vascular vessels with polyphenolic and lignin materials. Initial typical symptoms of VL infection in OSR are leaf chlorosis (one-sided or irregular yellowing) which is followed by senescence. During later disease development, yellow or brown longitudinal lesions are formed on stems and branches (Fig. 1.3). As plants mature, the fungus bursts out of the xylem vessels and produces microsclerotia, first in the pith and then underneath the epidermis causing stem and root pith tissues to turn dark greyish or black. This marks the beginning of the saprophytic stage. In contrast to other Verticillium species, VL causes no wilting possibly because of the absence of wilting toxins and/or the presence of sufficient xylem vessels unblocked by occlusions in infected plants (Dunker et al., 2008). Premature ripening and senescence of leaves, stems or branches are the typical symptoms (Gladders, 2009; Leino, 2006). Plants inoculated under greenhouse conditions show additional symptoms such as stunting of shoots, reduction of root length (Dunker et al., 2008) and excessive production of branches (Fig. 4.2). Further plant aging towards harvest promotes intense formation of microsclerotia underneath the stem epidermis, in the stem pith and roots. As diseased plants senescence, microsclerotia are released into the soil together with dead plant material. At this point, the pathogen enters the dormant stage (Fig. 1.3). 
Spreading of VL can occur via several mechanisms. Transport of non-symptomatic, but infected plant products and/or seeds can move the pathogen long distance. Once established in a field, spread of the pathogen occurs primarily by soil cultivation and movement of soil by wind, water or farm equipment (Gladders, 2009; Berlanger and Powelson, 2000).
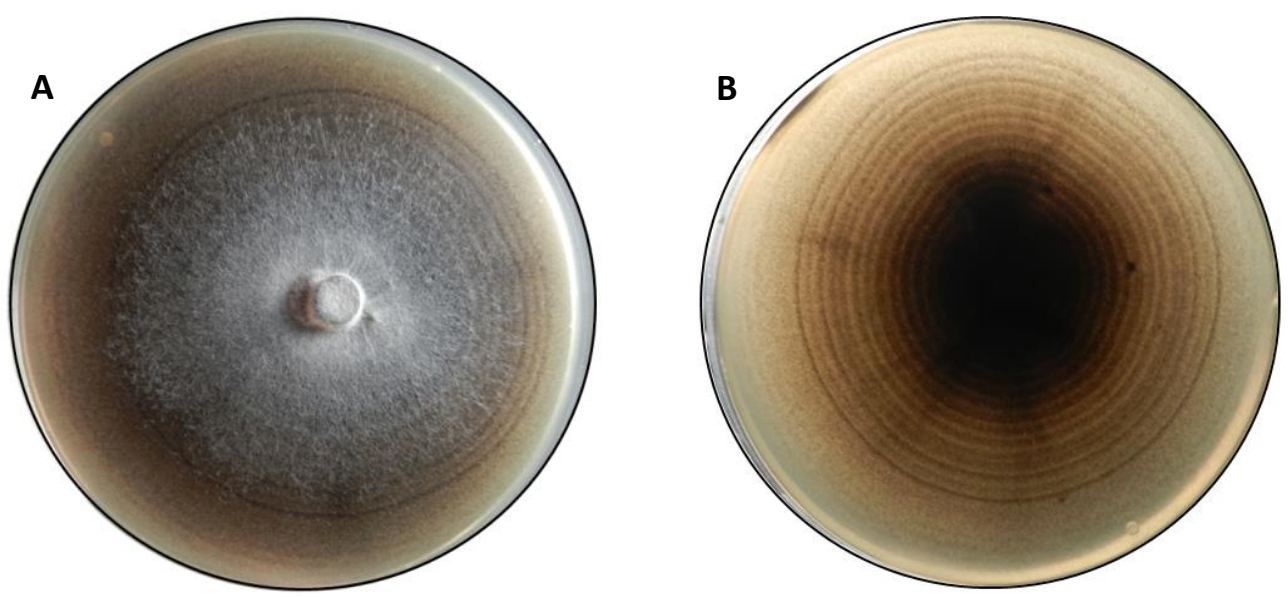

Figure 1.2 Growth of Verticillium longisporum isolate VL43 on potato dextrose agar plate four weeks after incubation at $23^{\circ} \mathrm{C}$ in the dark. A. Frontal view: flat white mycelial growth and balck microsclorotia produced over the whole plate. B. Bottom view: dark microsclerotia forming a ring pattern of growth.

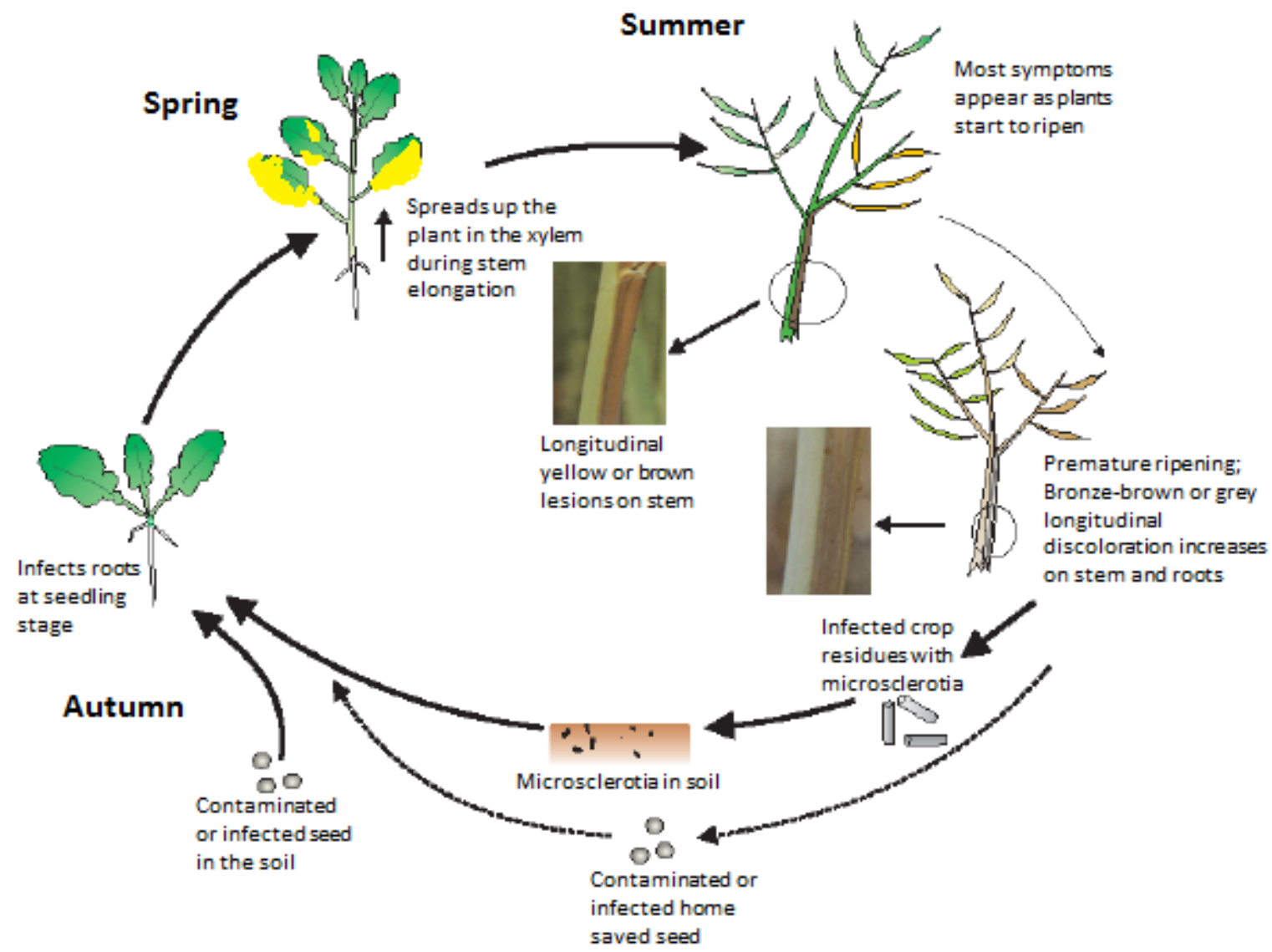

Figure 1.3 Disease cycle of Verticillium longisporum in winter oilseed rape (B. napus L.)

(Adapted from Gladders, 2009 and Leino, 2006) 


\subsubsection{Pathogenicity factors in V. longisporum}

Pathogenicity factors of VL are not yet exclusively known. Only few studies have shown the possible involvement of some genes or metabolites in infection of B. napus and Arabidopsis thaliana. For instance, Singh et al. (2010) have shown that silencing of a gene encoding chorismate synthase (Vlaro2), the first branch point intermediate of aromatic amino acid biosynthesis, caused a bradytrophic mutant that had reduced virulence in Arabidopsis and B. napus. Floerl et al. (2012) suggested rapid down-regulation and delayed induction of plant defence genes as possible mechanisms of enhanced virulence of VL in Arabidopsis. Singh et al. (2012) proposed increased expression of catalase peroxidase (VICPEA gene) and other oxidative stress response proteins in VL to protect the fungus from oxidative stress generated by B. napus. Timpner et al. (2013) have shown the significant role of the amino acid synthesis regulatory cross-pathway control system gene CPC1 in pathogenicity and colonization VL in B. napus. Production of pathogenesis related cell death and wilt inducing toxins are known from the closely related species $V$. dahliae (Xie et al., 2013) and $V$. albo-atrum (Mansoori and Smith, 2005). However, so far, there are no reports on production of pathogenesis related toxins by VL.

\subsubsection{Management of $V$. longisporum in OSR}

The characteristic systemic mode of infection and capability of long-term survival in soil makes Verticillium species difficult to control pathogens. As a result, despite the associated risks on the environment, control of Verticillium species in general has heavily relied on soil fumigation with chemicals (Klosterman et al., 2009). For VL in particular, no registered fungicides are currently available. Studies suggest that soil amendment with organic products or biological agents has the potential to reduce soil inoculum and may provide an effective suppression of Verticillium diseases. Nevertheless, the efficacy of this method is dependent on soil, climatic and agronomic factors. Moreover, there are some economic and ecological risks associated with this method of disease control (Goicoechea, 2009; França et al., 2013). The other possible alternative is crop rotation. Because of the existing host range specificity in the genus Verticillium, some crop rotation schemes that potentially minimize the risk of VL disease epidemics are suggested (Bhat and Subbarao, 1999; Zeise and Tiedemann, 2002). Interesting results from long-term field studies on the role of crop rotation in minimizing yield reduction in OSR due to fungal pathogen has been shown recently (Hilton et al., 2013). However, since Verticillium inoculum can remain viable in the soil for more than a decade (Wilhelm, 1955), the effectiveness of this option as a sole means of VL control is questionable. Although VL transmission via seeds is not a likely scenario (Zhou et al., 2006), seed treatment or the use of pathogen-free seed can minimize the risk of pathogen spread. For more effective quarantine however, accurate identification and knowledge on the identity of Verticillium species are essential (Inderbitzin and Subbarao, 2014). 
In general, until present, the use of plant resistance is the only feasible means for the management of VL in OSR. Breeding and resistance study efforts made in the last decade identified genotypes with enhanced VL resistance in OSR (Rygulla et al., 2007b; Eynck et al., 2009a) and cauliflower (Debode et al, 2005). Moreover, some of the resistance mechanisms in OSR and Arabidopsis are known. Among these, the major mechanisms of VL-resistance known in OSR are physical barriers (such as occlusions and cell wall bound lignin and phenolics) and other soluble phenolic compounds (Eynck et al., 2009b; Obermeier et al., 2013). Similarly, the significance of soluble phenylpropanoids in defence response of Arabidopsis towards VL is known (König et al., 2014). Another recent study on the Arabidopsis-VL interaction demonstrated the role of the Erecta gene (which encodes for a receptor-like kinase involved in plant development and disease resistance) in mediating resistance against VL-induced stunting in Arabidopsis (Häffner et al., 2014). Floerl et al. (2008) identified VLinduced enhanced accumulation of antifungal proteins in B. napus. Regarding plant hormones, despite the fact that VL-infection causes increased accumulation of salicylic acid, several studies have shown no role of this hormone in signalling VL resistance in Arabidopsis and B. napus (Veronese et al., 2003; Johansson et al., 2006; Ratzinger et al., 2009; Kamble et al., 2013).

\subsection{Aims of the thesis}

Even though much is known about the basics of VL resistance mechanisms in OSR, there is a lack of information regarding the nature of disease resistance under abiotic stress conditions. Siebold and Tiedemann (2013) recently demonstrated the potential effect of high soil temperature in causing early and severe VL infection in OSR. Besides this, a review on the impact of climate change on OSR diseases clearly showed a gap of knowledge on the influence of changing soil conditions on soilborne diseases of OSR including VL (Evans et al., 2009). This indicates the significance of understanding the nature of pathogen virulence, disease development and host resistance in the presence of prevailing abiotic stress conditions, particularly, drought and high temperature. With this background, the present thesis focused on a functional analysis of VL-resistance in OSR. Accordingly, several studies from the identification of VL resistant lines, towards further investigation of cultivar-related resistance mechanisms and the nature of plant resistance under drought stress conditions were conducted under various experimental conditions. The particular rationale behind each study is given in the different chapters. Here, the general objectives of the respective chapters are briefly described.

If plant resistance is to be used as one alternative means of disease management, the development or identification of plant genotypes with enhanced disease resistance is the first step. Accordingly, screening of $B$. napus lines for resistance against VL using molecular and phenotypic disease assessment tools was conducted in greenhouse, outdoor and field experiments. The major 
objectives of these experiments were to identify B. napus double haploid lines and other accessions with high level of resistance against VL. A further objective of this part of the thesis (Chapter 2) was validating the applicability of $\mathrm{qPCR}$ (quantitative polymerase chain reaction) as an alternative method of disease evaluation in the field.

In order to make practical use of plant resistance, resistance traits found from whatever source need to be transferred to a desired crop variety such as to high yielding cultivars. Among other things, the pre-requisite for successful transfer of these traits is the in-depth understanding of the resistance mechanisms in the host plant. This helps not only the easy and selective transfer of traits, but also provides a space to address specific agro-ecological requirements. As mentioned earlier, the role of some basic physical and biochemical resistance factors that work against VL are known in OSR. However, nothing is known regarding the existence and role of soluble, antifungal and cultivarrelated VL-resistance in the OSR xylem sap, an environment where the pathogen spends most part of its life cycle. To answer this important question, a study involving greenhouse experiments, in vitro bioassays and biochemical analyses was conducted using VL-susceptible and resistant genotypes. The general objective of this study (Chapter 3) was to find out whether xylem sap plays a major role in cultivar-related resistance of OSR against VL.

Since the effects of vascular pathogens (like VL) mimic the effects of other abiotic stress factors such as drought or high temperature, it is indispensable to understand what happens to host resistance to either of the stress factors particularly under conditions where both stresses occur simultaneously. This critical issue, with particular importance under conditions of changing global climate, was addressed in an extensive study with a general objective of investigating the main and interactive effects of VL infection and drought stress on VL and OSR. In this study (Chapter 4), the nature of pathogen development and host reaction towards both stress factors was investigated by analysing several phenotypic, physiological, molecular, agronomic and yield parameters. 


\subsection{References}

Agrios G. N. 2005. Plant pathology (5th edition). Elsevier-Academic Press. San Diego, CA.

Alford D. V., Nilsson C. and Ulber B. 2003. Insect pests of oilseed rape crops. In: Alford, D. V. (ed.), Bio-control of oilseed rape pests, 9-39. Blackwell Science, Oxford.

Berlanger I. and Powelson M. L. 2000. Verticillium wilt. The plant health instructor. Available: http://www.apsnet.org/edcenter/intropp/lessons/fungi/ascomycetes/Pages/VerticilliumWilt .aspx. doi: 10.1094/PHI-I-PHI-1-2000-0801-01. Updated 2005.

Bhat R. G. and Subbarao, K. V. 1999. Host range specificity in Verticillium dahliae. Phytopathology, 89:1218-1225.

CABI and EPPO, 2011. Verticillium longisporum. Distribution maps of plant diseases. Map No. 1117 (Edition 1). ISSN 0012-396X. CAB International, Wallingford, UK.

Carré P. and Pouzet A. 2014. Rapeseed market, worldwide and in Europe. Oilseeds and fats crops Lipids, 21 (1) D102.

Clewes E., Edwards S. G., and Barbara D. J. 2008. Direct molecular evidence supports long-spored microsclerotial isolates of Verticillium from crucifers being interspecific hybrids. Plant Pathology, 57:1047-1057.

Collins A., Okoli C. A. N., Morton A., Parry D., Edwards S. G., and Barbara D. J. 2003. Isolates of Verticillium dahliae pathogenic to crucifers are of at least three distinct molecular types. Phytopathology, 93:364-376.

Debode J., Declercq B. and Höfte M. 2005. Identification of cauliflower cultivars that differ in susceptibility to Verticillium longisporum using different inoculation methods. Journal of Phytopathology, 153:25-263.

Din J., Khan S. U., Ali I. and Gurmani A. R. 2011. Physiological and agronomic response of canola varieties to drought stress. The Journal of Animal \& Plant Sciences, 21(1):78-82.

Dunker S., Keunecke H., Steinbach P., von Tiedemann A. 2008. Impact of Verticillium longisporum on yield and morphology of winter oilseed rape (Brassica napus) in relation to systemic spread in the plant. Journal of Phytopathology 156: 698-707.

Evans N., Gladders P., Fitt B.D.L. and von Tiedemann V. 2009. Climate change in Europe : altered life cycles and spread of major pathogens in oilseed rape. In GCIRC technical meeting. GCIRC technical meeting, New Delhi, India, 2-4 February .

Eynck C., Koopmann B. and von Tiedemann A. 2009a. Identification of Brassica accessions with enhanced resistance to Verticillium longisporum under controlled and field conditions. Journal of Plant Diseases and Protection, 116(2), 63-72.

Eynck C., Koopmann B., Grunewaldt-Stoecker G., Karlovsky P. and von Tiedemann A. 2007. Differential interactions of Verticillium longisporum and $V$. dahliae with Brassica napus 
detected with molecular and histological techniques. European Journal of Plant Pathology, 118:259-274.

Eynck C., Koopmann B., Karlovsky P. and von Tiedemann A. 2009b. Internal resistance in winter oilseed rape inhibits systemic spread of the vascular pathogen Verticillium longisporum. Phytopathology, 99:802-811.

Fahleson J., Hu Q. and Dixelius C. 2004. Phylogenetic analysis of Verticillium species based on nuclear and mitochondrial sequences. Archives of Microbiology, 181:435-442.

Fahleson J., Lagercrantz U., Hu Q., Steventon L. A. and Dixelius C. 2003. Estimation of genetic variation among Verticillium isolates using AFLP analysis. European Journal of Plant Pathology, 109:361-371.

Floerl S., Majcherczyk A., Possienke M., Feussner K., Tappe H, Gatz C., Feussner I., Kües U. and Polle A. 2012. Verticillium longisporum infection affects the leaf apoplastic proteome, metabolome, and cell wall properties in Arabidopsis thaliana. PLOS ONE 7(2): e31435.

França S. C., Spiessens K., Pollet S., Debode J., De Rooster L., Callens D. and Höfte M. 2013. Population dynamics of Verticillium species in cauliflower fields: Influence of crop rotation, debris removal and ryegrass incorporation. Crop Protection, 54:134-141.

Gabor B., John K. and Diane K. 2013. In Conn K. E. and Rosenberger S. A. (eds). Crucifer disease reference guide. A practical guide for seedsmen, growers and agricultural advisors. Seminis vegetable seeds. CA, USA.

Gianessi L., Sankula S. and Reigner N. 2003. Plant biotechnology: Potential impact for improving pest management in European agriculture. oilseed rape-herbicide-tolerant case study. The National Center for Food and Agricultural Policy. DC, USA.

Gladders P. 2009. Relevance of Verticillium wilt (Verticillium longisporum) in winter oilseed rape in the UK. HCGA Research Review, 72:1-39.

Goicoechea N. 2009. To what extent are soil amendments useful to control Verticillium wilt? Pest Management Science, 65:831-839.

Gómez-Campo C. 1999. Biology of Brassica coenospecies. Developments in plant genetics and breeding, 4. Elsevier, Amsterdam/New York.

Häffner E., Karlovsky P., Diederichsen E. 2010. Genetic and environmental control of the Verticillium syndrome in Arabidopsis thaliana. BMC Plant Biology,14.85:1-16.

Häffner E., Karlovsky P., Splivallo R., Traczewska A. and Diederichsen E. 2014. ERECTA, salicylic acid, abscisic acid, and jasmonicacid modulate quantitative disease resistance of Arabidopsis thaliana to Verticillium longisporum. BMC Plant Biology, 14:85.

Haramoto E. R. and Gallandt E. R. 2005. Brassica cover cropping: I. Effects on weed and crop establishment. Weed Science, 53(5):695-701. 
Haramoto E. R. and Gallandt E. R. 2004. Brassica cover cropping for weed management: A review. Renewable Agriculture and Food Systems, 19: 187-198

Hilton S., Bennett A.J., Keane G., Bending G.D., Chandler D., Stobart R. and Mills P. 2013. Impact of shortened crop rotation of oilseed rape on soil and rhizosphere microbial diversity in relation to yield decline. PLOS ONE, 8(4): e59859.

Inderbitzin P. and Subbarao K. V. 2014. Verticillium systematics and evolution: How confusion impedes Verticillium wilt management and how to resolve it. Phytopathology, 104:564-574.

Inderbitzin P., Bostock R.M., Davis R. M., Usami T., Platt H. W. and Subbarao K. V. 2011b. Phylogenetics and taxonomy of the fungal vascular wilt pathogen Verticillium, with the descriptions of five new species. PLOS ONE, 6(12): e28341.

Inderbitzin P., Davis R. M., Bostock R. M. and Subbarao K. V. 2011a. The Ascomycete Verticillium longisporum is a hybrid and a plant pathogen with an expanded host range. PLOS ONE, 6(3): e18260.

Inderbitzin P., Davis R.M., Bostock R. M., Subbarao K.V . 2013. Identification and differentiation of Verticillium species and $V$. longisporum lineages by simplex and multiplex PCR assays. PLoS ONE, 8(6): e65990.

Ingram R. 1968. Verticillium dahliae var. longisporum, a stable diploid. Transactions of the British Mycological Society, 51: 339-341.

Isaac I. 1957. Verticillium wilt of Brussels sprout. Annals of Applied Biology, 45:276-283.

Johansson A., Goud Jan-K. C. and Dixelius C. 2006. Plant host range of Verticillium longisporum and microsclerotia density in Swedish soils. European Journal of Plant Pathology, 114:139-149.

Kamble A., Koopmann B. and von Tiedemann A. 2013. Induced resistance to Verticillium longisporum in Brassica napus by b-aminobutyric acid. Plant Pathology, 62:552-561.

Karapapa V. K., Bainbridge B. W. and Heale J. B. 1997. Morphological and molecular characterization of Verticillium longisporum comb. nov., pathogenic to oilseed rape. Mycological Research, 101:1281-1294.

Klosterman S. J., Atallah Z. K., Vallad G. E. and Subbarao K. V. 2009. Diversity, pathogenicity, and management of Verticillium Species. Annual Review of Phytopathology. 47:39-62.

König S., Feussner K., Kaever A., Landesfeind M., Thurow C., Karlovsky P., Gatz C., Polle A. and Feussner I. 2014. Soluble phenylpropanoids are involved in the defense response of Arabidopsis against Verticillium longisporum. New Phytologist , 202:823-837.

Lane A. and Gladders P. 2000. Pests and diseases of oilseeds, Brassica seed crops and field beans. In: Alford D. V. (ed): Pest and disease management handbook. Blackwell science: Oxford.

Lardon A., and Triboi-Blondel A.M. 1995. Cold and freeze stress at flowering Effects on seed yields in winter rapeseed. Field Crops Research, 44:95-101. 
Leino M.2006. Fungal diseases on oilseed rape and turnip rape. Norrköping, Sweden.

Mansoori B. and Smith C. J. 2005. Verticillium-toxins: their role in pathogenesis. Journal of Agricultural Science and Technology, 7: 103-114.

Messner R., Schweigkofler W., Ibl M ., Berg G . and Prillinger H.. 1996. Molecular characterization of the plant pathogen Verticillium dahliae Kleb. using RAPD-PCR and sequencing of the 18SrRNA-gene. Journal of Phytopathology, 144: 347-354.

Obermeier C., Hossain M. A., Snowdon R., Knüfer J., von Tiedemann A. and Friedt W. 2013. Genetic analysis of phenylpropanoid metabolites associated with resistance against Verticillium longisporum in Brassica napus. Molecular Breeding, 31:347-361.

OECD. 1997. Consensus document on the biology of Brassica napus L. (Oilseed rape). Series on harmonization of regulatory oversight in biotechnology, No.7. Organization for economic cooperation and development (OECD), Paris.

OGTR. 2002. The biology and ecology of canola (Brassica napus). Australian government, office of the gene technology regulator, Canberra, Australia.

Orlovius K. 2003. Oilseed rape. In: Kirbky, E. A. (Ed.).Fertilizing for high yield and quality, Bulletin 16. International potash institute. Basel, Switzerland.

Pantou M. P., Strunnikova O. K., Shakhnazarova V. Yu., Vishnevskaya N. A., Papalouka V. G. and Typas M. A.. 2005. Molecular and immunochemical phylogeny of Verticillium species. Mycological Research, 109:889-902.

Polowick P. L. and Sawhney V. K. 1988. High temperature induced male and female sterility in canola (Brassica napus L). Annals of Botany 62, 83-86.

Ratzinger A., Riediger N., von Tiedemann A. and Karlovsky P. 2009. Salicylic acid and salicylic acid glucoside in xylem sap of Brassica napus infected with Verticillium longisporum. Journal of Plant Research, 122:571-579.

Roos J. 2014. Verticillium longisporum and plant immunity responses in Arabidopsis. PhD thesis. Swedish University of Agricultural Sciences. Uppsala, Sweden.

Rygulla W., Snowdon R. J., Eynck C., Koopmann B., von Tiedemann A., Lühs W. and Friedt W. 2007b. Broadening the genetic basis of Verticillium longisporum resistance in Brassica napus by interspecific hybridization. Phytopathology, 97:1391-1396.

Sadaqat H. A., Tahir M. H. N. and Hussain M. T. 2003. Physiogenetic aspects of drought tolerance in canola (Brassica napus).International Journal of Agriculture and Biology, 5:(4) 611-614.

Sattell R., Dick R., Ingham R., Karow R., Kaufman D. and D. McGrath. 1998. Rapeseed (Brassica campestris/Brassica napus). Oregon State University, USA.

Siebold M. and von Tiedemann A. 2013. Effects of experimental warming on fungal disease progress in oilseed rape. Global Change Biology, 19:1736-1747. 
Sinaki J. M., Heravan E.M., Rad A.S.H., Noormohammadi G. and Zarei G. 2007. The effects of water deficit during growth stages of canola (Brassica napus L.). American-Eurasian Journal of Agricultural \& Environmental Sciences, 2(4):417-422.

Singh S., Braus-Stromeyer S. A., Timpner C., Valerius O., von Tiedemann A., Karlovsky P., Druebert C., Polle A. and Braus G. H. 2012. The plant host Brassica napus induces in the pathogen Verticillium longisporum the expression of functional catalase peroxidase which is required for the late phase of disease. Molecular Plant-Microbe Interactions, 25(4):569-581.

Singh S., Braus-Stromeyer S. A., Timpner C., Tran Van T., Lohaus G., Reusche M., Knüfer J., Teichmann T., von Tiedemann A., Braus G. H. 2010. Silencing of Vlaro2 for chorismate synthase revealed that the phytopathogen Verticillium longisporum induces the crosspathway control in the xylem. Applied Microbiology and Biotechnology, 85:1961-1976.

Snowdon R. J., Luhs W. and Friedt W. (2007) Oilseed rape. In: C. Kole. Genome mapping and molecular breeding in plants Volume 2. Springer, Heidelberg. pp. 55-114.

Song K. and Osborn T. C. 1992. Polyphyletic origins of Brassica napus: new evidence based on organelle and nuclear RFLP analyses. Genome, 35(6): 992-1001.

Steventon L. A., Fahleson J., Hu Q. and Dixelius C. 2002. Identifcation of the causal agent of Verticillium wilt of winter oilseed rape in Sweden, V. Iongisporum. Mycological Research, 106(5): 570-578.

Timpner C., Braus-Stromeyer S. A., Tran Van T. and G. H. Braus. 2013. The Cpc1 regulator of the cross-pathway control of amino acid biosynthesis is required for pathogenicity of the vascular pathogen Verticillium longisporum. Molecular Plant-Microbe Interactions, 26(11):1312-1324.

Tran Van T., Braus-Stromeyer S. A., Timpner C. and Braus G. H. 2013. Molecular diagnosis to discriminate pathogen and apathogen species of the hybrid Verticillium longisporum on the oilseed crop Brassica napus. Applied Microbiology and Biotechnology, 97:4467-4483.

Veronese P., Narasimhan M. L., Stevenson R.A., Zhu J. K., Weller S. C., Subbarao K. V., Bressan R. A. 2003. Identification of a locus controlling Verticillium disease symptom response in Arabidopsis thaliana. The Plant Journal, 35(5):574-87.

Warwick S. I. and Al-Shehbaz I. A. 2006. Brassicaceae: Chromosome number index and database on CD-Rom. Plant Systematics and Evolution, 259:237-248.

Warwick S. I., Francis A., Gugel R. K. 2009. Taxonomic checklist and life history, ecological, and geographical data. In: Warwick S.I., Francis A., Gugel R.K. (ed.). Guide to wild germplasm of Brassica and allied crop (tribe Brassiceae, Brassicaceae). $3^{\text {rd }}$ Edition.

Wilhelm S. 1955. Longevity of the Verticillium wilt fungus in the laboratory and in the field. Phytopathology, 45:180-181. 
Xie C., Wang C., Wang X. and Yang X. 2013. Proteomics-based analysis reveals that Verticillium dahliae toxin induces cell death by modifying the synthesis of host proteins. Journal of General Plant Pathology, 79:335-345.

Zeise K. and von Tiedemann A. 2001. Morphological and physiological differentiation among vegetative compatibility groups of Verticillium dahliae in relation to $V$. longisporum. Journal of Phytopathology, 149,469-475.

Zeise K. and von Tiedemann A. 2002. Host specialization among vegetative compatibility groups of Verticillium dahliae in Relation to Verticillium longisporum. Journal of Phytopathology, 150, 112-119.

Zhou L., Hu Q., Johansson A. and Dixelius C. 2006. Verticillium longisporum and V. dahliae: infection and disease in Brassica napus. Plant Pathology, 55:137-144.

Zhou W. and Lin X. 1995. Effects of waterlogging at different growth stages on physiological characteristics and seed yield of winter rape (Brassica napus L.). Field Crops Research, 44(23):103-110. 


\section{Phenotypic and molecular evaluation of Brassica napus lines for Verticillium longisporum resistance under greenhouse, outdoor and field conditions}

Parts of this chapter have been published in: Knüfer, J. ${ }^{1,3}$, Lopisso, D. T. ${ }^{1}$, Koopmann, B. ${ }^{1}$, Karlovsky $P^{2}{ }^{2}$ and von Tiedemann $A^{1}$. 2016. Assessment of latent infection with Verticillium longisporum in field-grown oilseed rape by qPCR. Eur J Plant Pathol. doi:10.1007/s10658-016-1045-0.

${ }^{1}$ Department of Crop Sciences, Section of Plant Pathology and Crop Protection, Georg August University, Grisebachstr. 6, 37077 Göttingen, Germany; ${ }^{2}$ Department of Crop Sciences, Section of Molecular Phytopathology and Mycotoxin Research, Georg August University, Grisebachstr. 6, 37077 Göttingen, Germany; ${ }^{3}$ Current address: Strube Research GmbH \& Co. KG, Hauptstraße 1, 38387 Söllingen, Germany.

\subsection{Introduction}

Oilseed rape (OSR) is the world's second most produced oilseed behind soybeans (Carré and Pouzet, 2014). It is a multipurpose crop which is predominantly grown for vegetable oil and biodiesel (Orlovius, 2003), but also has gained large importance as a protein source for feed. OSR is a key component in crop rotation serving as a "catch crop" and ground cover over winter. It suppresses weed growth and loosens plow pans to help improve soil tilth (Sattell et al., 1998). Apart from this, OSR flowers provide an early spring period source of nectar and pollen for honeybees (Farkas and Zajácz, 2007). Due to the increasing importance of this crop, the global production has undergone sustained growth over the past two decades (Carré and Pouzet, 2014). Nevertheless, the increasing production of OSR is not without problems. Several diseases and insect pests are known to hamper its productivity. The recently evolved fungal vascular pathogen Verticillium longisporum (Inderbitzin et al., 2011) is one of the most important biotic constraints with high potential of threatening OSR production, particularly in areas with intense production of OSR (Carré and Pouzet, 2014), notably in Sweden (Johansson et al., 2006), Germany (Dunker et al., 2008), France and Poland (Karapapa et al., 1997) and quite recently in the UK (Gladders, 2009).

Verticillium longisporum (VL) is a host-specialized pathogen of crucifers (Karapapa et al., 1997; Zeise and von Tiedemann, 2002). In oilseed rape, it causes premature senescence and ripening which can cause up to $60 \%$ yield reduction (Dunker et al., 2008; Gladders, 2009). Inaccessibility of Verticillium species during infection and their long-term field persistence make them difficult to control. As a result, control of Verticillium diseases strongly relies on fumigation of soil with chemicals (Klosterman et al., 2009; Goicoechea, 2009). For V. longisporum in particular, no registered or effective fungicides are available. Because of its narrow host range characteristics, long-term crop rotations that likely decline the abundance of viable soil microsclerotia are suggested as an alternative measure for the control of VL (Rygulla et al., 2007; Gladders, 2009). Nevertheless, this 
option seems to be largely ineffective (França et al., 2013) mainly because of the long survival (up to 15 years) of the pathogen with its microsclerotia in soil (Berg et al., 1999; Leno, 2006). As a result, for the control of Verticillium diseases, the use of host-plant resistance remains the preferred strategy (Klosterman et al., 2009). The integration of such an easily adopted and environmentally safe method of disease management in crop protection packages provide several economic and ecological advantages (Agrios, 2005).

Regarding host-plant resistance against $V$. longisporum, elevated levels of resistance to VL were identified in cabbage (B. oleracea) genotypes a decade ago (Happstadius et al., 2003). Later on, by crossing tolerant $B$. oleracea accessions with $B$. rapa, resynthesized $B$. napus lines with enhanced VL resistance were developed (Happstadius et al., 2003; Rygulla et al., 2007a; Rygulla et al., 2007b). Previous greenhouse and field evaluation also identified promising $B$. napus lines with significantly higher level of resistance against VL (Rygulla et al., 2007b; Eynck et al., 2009a). In the present study VL resistance of winter OSR double haploid $(\mathrm{DH})$ lines generated from resistant resynthesized $B$. napus lines and other sets of $B$. napus accessions obtained from several private plant breeding companies were evaluated in greenhouse, outdoor and multi-site field trials. This study was conducted as part of a joint project on improving OSR resistance to $V$. longisporum. The project involved three partners, the University of Göttingen, Division of Plant Pathology and Crop Protection, the Justus-Liebig-University of Gießen, Department of Plant Breeding (JLU) and nine plant breeding companies (Table 2.1) through their association called GFP (an association for the promotion of private plant breeding in Germany). The major objectives of this part of the project was to identify best performing and highly VL-resistant lines for use in future breeding programs aiming at integration of VL-resistance in commercial high-yielding winter OSR varieties. Furthermore, the applicability of quantitative PCR (qPCR) as a method of VL disease assessment under field conditions was validated.

\subsection{Materials and methods}

\subsubsection{Greenhouse screening}

Greenhouse screening was carried out in four rounds of experiments (Appendix 2.1). The experiments were conducted during the periods from February 2011 to January 2014 in the Division of Plant Pathology and Crop Protection, Göttingen University, Germany.

\subsubsection{Plant material}

More than 230 winter oilseed rape lines obtained from different breeding companies (Table 2.1) were screened for Verticillium longisporum resistance under greenhouse conditions over three 
years. The descriptions and sources for all lines are indicated in Appendix 2.1. During each screening run, two reference varieties, Falcon (susceptible) and Express (resistant) were used as a check.

\subsubsection{Experimental design}

The experiment was arranged in a completely randomized design (CRD). After inoculation, two seedlings were grown in one pot $(200 \mathrm{ml})$ and considered as one replication. Each treatment was replicated 10 times resulting in 40 plants ( 20 mock inoculated and 20 VL-inoculated).

Table 2.1 Summary of Brassica napus lines screened for resistance against Verticillium longisporum in greenhouse and field experiments.

\begin{tabular}{ccc} 
Seed company & $\begin{array}{c}\text { Number of lines screened for VL resistance } \\
\text { Field trial }\end{array}$ & GH screening \\
\hline DM & 2 & - \\
DSV & 25 & 38 \\
JLU & 22 & - \\
KWS & 3 & 30 \\
NICK & 3 & 18 \\
NPZ & - & 23 \\
SRG & - & 22 \\
SW & 22 & 36 \\
SYN & 3 & 39 \\
WVB & - & 30 \\
\hline Total & 80 & 236
\end{tabular}

DM, Dieckmann GmbH \& Co. KG. DSV, Deutsche Saatveredelung AG. JLU, Justus-Liebig-University of Gießen, Department of Plant Breeding. KWS, KWS SAAT AG. NICK, Limagrain GmbH. NPZ, NorddeutschePflanzenzucht Hans-Georg Lembke KG. SRG, Raps GbR Saatzucht Lundsgaard. SW, Lantmännen SW Seed. SYN, Syngenta Seeds GmbH. WVB, W. von Borries-Eckendorf GmbH \& Co. VL, Verticillium longisporum.

\subsubsection{Inoculation procedures and disease scoring}

Seeds of $B$. napus lines were double surface sterilized with $70 \%$ ethanol and grown on sterile silica sand for 10 days under optimum conditions $\left(20^{\circ} \mathrm{C}\right.$ temperature, $16 \mathrm{~h}$ light and an average relative humidity of $60 \%)$. Spore suspension $\left(10^{6}\right.$ conidia $\left./ \mathrm{ml}\right)$ was prepared from ten days old cultures of the highly virulent $V$. longisporum isolates VL43 and VL40 mixed in equal ratio (See section 4.2.4 for details). Inoculation was performed following the cut root-dip inoculation method as described in section 4.2.4. Inoculated seedlings were transferred to $200 \mathrm{ml}$ plastic pots (two plants per pot) filled with a substrate composed of sand, peat and compost (1:1:2) and grown under optimum conditions as described above. Two disease assessment methods (disease severity and stunting effects) were used. Evaluation of disease severity was performed on a weekly basis for four consecutive weeks (728 days post inoculation, DPI). Disease severity was scored using a standard VL disease index (Table 
4.2). From these disease index recordings, AUDPC and net AUDPC values were calculated as described in section 4.2.7.1. To evaluate stunting effects, plant height of VL and mock-inoculated plants was measured at $28 \mathrm{DPI}$ and the percentage of relative stunting due to VL was calculated as $\left[\left(h_{\text {Mock }}-h_{V L}\right) \times(100)\right] /\left[\left(h_{\text {Mock }}\right)\right]$, where $h_{\text {Mock }}$ and $h_{V L}$ are the plant heights of mock and VL inoculated treatments, respectively. In order to standardize fluctuating levels of infection during different screenings, normalization of net AUDPC was done by dividing the net AUDPC values of each line to that of the average values of the reference cultivars. That is, normalized AUDPC ( AUDPC $\left._{\text {norm }}\right)=[$ Net $\left.A U D P C_{\text {lines }}\right] /\left[\left(\right.\right.$ Net $A U D P C_{\text {Falcon }}+$ Net $\left.\left.A U D P C_{\text {Express }}\right) / 2\right]$. Normalized percent relative stunting was calculated in the same way as for net AUDPC values (Eynck et al, 2009a).

\subsubsection{Field trials}

Field studies were conducted with the aim of verifying resistance of $B$. napus accessions under natural conditions, with diversity in terms of soil, climate and disease factors. For this trial, B. napus lines were used that showed a reasonably better resistance than the reference standard varieties under greenhouse conditions. The field experiments were conducted in three locations (Table 2.2) and for three seasons (2010/11, 2011/12 and 2012/13).

\subsubsection{Plant materials}

A total of 80 lines (Table 2.1) selected on the basis of previous greenhouse screening results were evaluated under field conditions. Of these, 61 were double haploid (DH) lines derived from resynthesized VL-resistant B. napus lines. The remaining 19 lines with undisclosed genetic backgrounds were obtained from different breeding companies. Fourteen lines (thirteen DH lines and one accession) were repeatedly tested for three consecutive growing seasons across all locations (Appendix 2.2). During each trial, 40 lines (including the reference varieties) were tested. Cultivars Falcon, Laser, Oase, and Express were used as a standard check. Detailed descriptions and sources of all plant materials are found in (Appendix 2.2). Similar seed lots of all lines tested in the field were used in the parallel evaluation in the greenhouse.

\subsubsection{Description of experimental locations}

The three locations with field trials were Göttingen (Northern Central Germany), Fehmarn (an island in the Baltic Sea, Northern Germany) and Svalöv (Southern Sweden). These locations were selected on the basis of variation in the level of natural disease infestation, climatic factors and soil conditions (Table 2.2).

\subsubsection{Experimental design}

All experiments were laid down in a randomized complete block design (RCBD) with two (Fehmarn and Svalöv) or four (Göttingen) replications. In all locations, 40 accessions including the reference 
varieties were tested during each growing season. Experimental plot size was $8 \mathrm{~m} \times 1.5 \mathrm{~m}$ with eight rows. Distance between blocks was kept 0.3 meter apart. Plant samples harvested from the central two rows were used for data recordings.

Table 2.2 Field characteristics of the three research locations where field studies were conducted from 2011 to 2013.

\begin{tabular}{|c|c|c|c|c|c|c|c|c|}
\hline Location & Site name & Latitude & Alt (mas) & $\mathrm{T}\left({ }^{\circ} \mathrm{C}\right)$ & $\mathrm{RF}(\mathrm{mm})$ & $\mathrm{RH}(\%)$ & ST & VL-NI \\
\hline \multicolumn{9}{|l|}{ Göttingen } \\
\hline 2010/11 & Große Breite & $51^{\circ} 33^{\prime} \mathrm{N} 9^{\circ} 54^{\prime} \mathrm{E}$ & 138 & 7.8 & 463.2 & 78.0 & Loam & None/Low ${ }^{182}$ \\
\hline $2011 / 12$ & Große Breite & $51^{\circ} 33^{\prime} \mathrm{N} 9^{\circ} 54^{\prime} \mathrm{E}$ & 138 & 9.3 & 567.8 & 73.0 & Loam & None/Low ${ }^{182}$ \\
\hline $2012 / 13$ & Rosdorf & $51^{\circ} 30^{\prime} \mathrm{N} 9^{\circ} 54^{\prime} \mathrm{E}$ & 169 & 8.3 & 438.8 & 85.7 & Loam & None/Low ${ }^{182}$ \\
\hline \multicolumn{9}{|l|}{ Fehmarn } \\
\hline 2010/11 & Niendorf & $54^{\circ} 27^{\prime} \mathrm{N} 11^{\circ} 12^{\prime} \mathrm{E}$ & ND & 8.6 & 687.2 & ND & ND & $\mathrm{High}^{1 \& 2}$ \\
\hline $2011 / 12$ & Niendorf & $54^{\circ} 27^{\prime} \mathrm{N} 11^{\circ} 12^{\prime} \mathrm{E}$ & ND & 9.3 & 671.1 & ND & ND & $\mathrm{High}^{182}$ \\
\hline $2012 / 13$ & Niendorf & $54^{\circ} 27^{\prime} \mathrm{N} 11^{\circ} 12^{\prime} \mathrm{E}$ & ND & 8.6 & 481.7 & ND & ND & $\operatorname{High}^{182}$ \\
\hline \multicolumn{9}{|l|}{ Svalöv } \\
\hline 2010/11 & Svalöv & $55^{\circ} 54^{\prime} \mathrm{N} 13^{\circ} 6^{\prime} \mathrm{E}$ & ND & 6.9 & 830.4 & 86.7 & ND & Very high ${ }^{283}$ \\
\hline $2011 / 12$ & Svalöv & $55^{\circ} 54^{\prime} \mathrm{N} 13^{\circ} 6^{\prime} \mathrm{E}$ & ND & 8.1 & 703.2 & 81.1 & ND & Very high ${ }^{283}$ \\
\hline $2012 / 13$ & Svalöv & $55^{\circ} 54^{\prime} \mathrm{N} 13^{\circ} 6^{\prime} \mathrm{E}$ & ND & 7.2 & 594.4 & 80.0 & ND & Very high ${ }^{2 \& 3}$ \\
\hline
\end{tabular}

Key: Alt, Altitude. mas, meters above sea level. T, mean annual temperature. RF, Total annual rainfall. RH, Average annual relative humidity. ST, soil type. ND, data not available. VL-NI, level of natural Verticillium longisporum infestation. For Göttingen and Svalöv sites the mean weather data from August to July (time period between sowing and harvest of oilseed rape) was obtained from data logger instruments installed inside the trial sites. For Fehmarn regional weather data was obtained from http://www.wetterkontor.de. ${ }^{1}$ Knüffer, 2013; ${ }^{2}$ Eynck, 2008; ${ }^{3}$ Johansson et al., 2006.

\subsubsection{Inoculation and agronomic practices}

At Svalöv and Fehmarn, where there is a reasonably high level of inoculum in the soil (Eynck, 2008; Johansson, 2006), experiments were carried out under natural infestation conditions. In Göttingen, artificial inoculation was performed using chopped VL-infected stubbles collected from previous year experiments. This was done by evenly spreading the inoculum on a prepared seedbed at a rate of $15 \mathrm{~g}$ microsclerotia infested stubbles $/ \mathrm{m}^{2}$. The inoculum was applied just before sowing and it was thoroughly incorporated into the soil using a disk plough. Seed rate was adjusted to local recommendations i.e. 50, 60 and 80 seeds $/ \mathrm{m}^{2}$ in Fehmarn, Göttingen and Svalöv, respectively. Early season insect pests (such as aphids and flea beetles) and seed borne pathogens (Phoma lingam, Alternaria spp. and Pythium spp.), were controlled by seed treatment with 'CRUISER OSR' (280 g/l thiamethoxam, $8 \mathrm{~g} /$ / fludioxonil and $32.3 \mathrm{~g} / \mathrm{I}$ metalaxyl-M; Syngenta Crop Protection UK Limited) at a rate of $0.02 \mathrm{ml}$ per gram of seed. All other agronomic practices including fertilization and spraying 
against weeds, blackleg (Phoma lingam) and stem rot (Sclerotinia sclerotiorum) were applied following standard recommendations.

\subsubsection{Disease assessment}

To measure VL-resistance of selected B. napus lines under field conditions, molecular and phenotypic disease assessment tools were used. For molecular disease measurements, stem samples were collected at the beginning of the ripening stage ( $\mathrm{BBCH} 80)$ and from these samples, fungal biomass was quantified by qPCR. For this protocol, sampling was done by taking $10 \mathrm{~cm}$ long stem sections cut approximately $5 \mathrm{~cm}$ above the base of the plant (Knüfer, 2013). For each accession, 10 stem samples were collected from each replication. Genomic DNA was extracted from 1 gram of lyophilized and ground stem sample taken from a pool of 10 stem samples. All other subsequent qPCR analysis steps including extraction of genomic DNA, DNA quality check, preparation of VL DNA standards, primers and qPCR conditions were similarly performed as described in section 4.2.7.3.

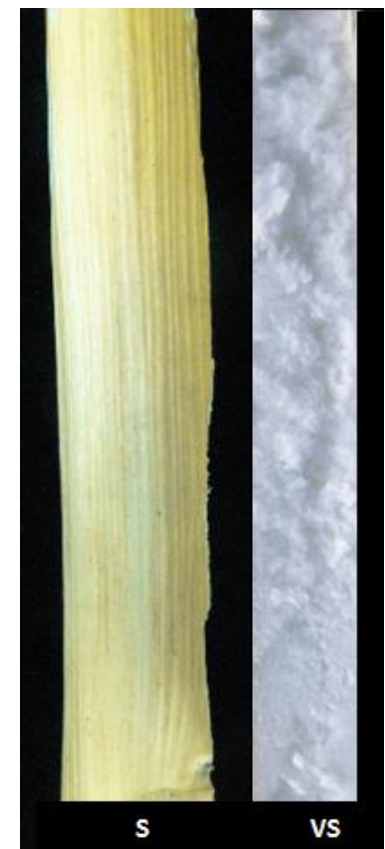

1

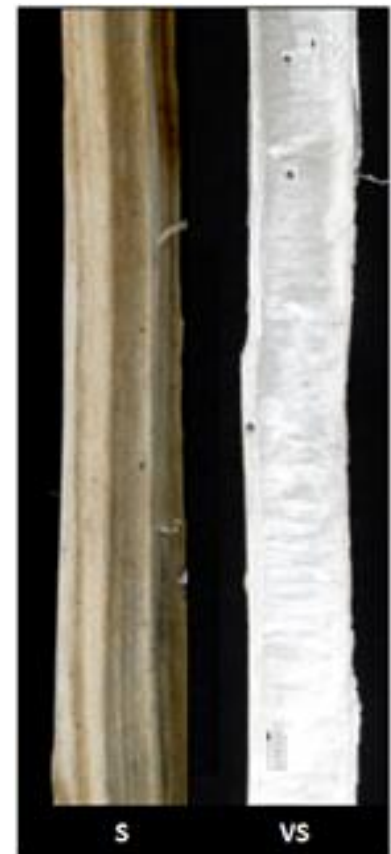

2

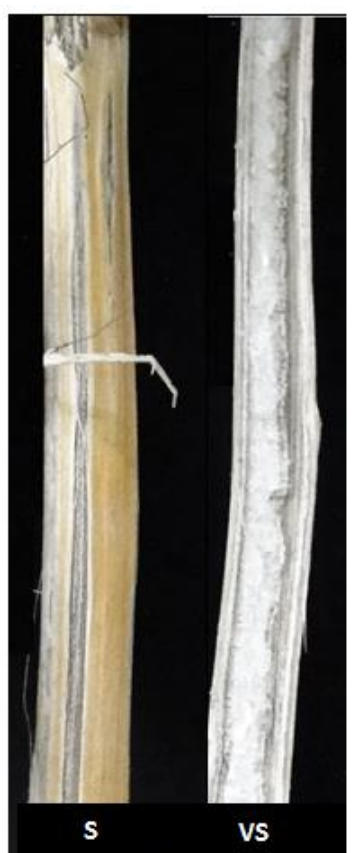

3

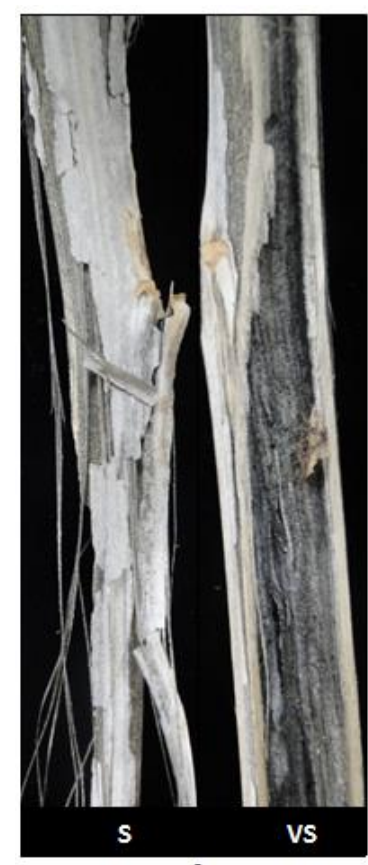

4

Figure 2.1 Assessment key (1-4) for evaluation of Verticillium longisporum disease severity on stubble and root of oilseed rape (Brassica napus). 1. Healthy tissue: Surface free of any symptoms and fungal structures; pith white and with cottony appearance; no microsclerotia visible. 2. Slight infestation: Narrow and short longitudinal lesions on the surface; pith still whitish but a few patches of microsclerotia visible. 3. Advanced infestation: Very long lesions and abundant microsclerotia visible under the epidermis; epidermis peeling off; long patches of black microsclerotia visible in the pith. 4. Severe infestation: Most part of the stem epidermis peeling off and disintegrated; the surface black and covered with microsclerotia; pith becomes dark, disintegrated and filled with microsclerotia. S, stem surface. VS, vertical section.

In order to identify the most suitable stage of plant growth at which VL can be detected (quantified) in stem tissue of field grown plants and to precisely know the plant stage at which field grown susceptible and resistant plants can be best differentiated using this method, VL biomass from the 
four reference varieties was quantified at three different growth stages. This study was conducted in $2012 / 13$. For this study, stem samples from the central four rows of each plot were collected at $\mathrm{BBCH} 65,70$, and 80 representing $50 \%$ flowering, end of flowering, and beginning of ripening stages, respectively. For each variety, 40 stem samples representing four biological replicates (10 plant samples per replication) were collected. In Göttingen, since there were four replications, sampling was done by collecting 10 stem samples per plot. In Fehmarn and Svalöv sites, since there were two replications, sampling was done by dividing each plot into two halves and collecting 10 stem samples from each part.

For phenotypic disease assessment, stubble sample collected after harvest was evaluated for stem and root disease severity. For every season study, a total of 50 (Fehmarn and Svalöv) or 100 (Göttingen) stubble samples were collected from each line (i.e 25 samples per replication). Disease severity evaluation was carried out by visual assessment of VL symptoms as well as microscopic estimation of the amount of microsclerotia both on the stem surface as well as in the pith tissue of stem and root samples using a four scale disease index (Fig. 2.1; Appendix 2.6).

\subsubsection{Outdoor experiment}

Unlike field conditions, infection of OSR with $V$. longisporum in greenhouse conditions typically induces clear symptoms of stunting and excessive production of side branches (see 4.4.1). Previous studies revealed weak or no correlation between results of field disease index and greenhouse AUDPC or stunting parameters (Knüfer, 2013; Eynck et al, 2009a). The possible reasons for such discrepancies in symptom development and resistance reactions between field and greenhouse experiments might arise from climatic variations, the type or amount of inoculum used, or even from the method of inoculation. In order to verify this hypothesis, B. napus lines used in the field trial 2011/12 were simultaneously tested under outdoor conditions with potted plants, which represent an intermediate situation between greenhouse and field conditions. Accordingly, inoculum source and method of inoculation were used in the same way as in the greenhouse screening experiments. To represent field conditions, vernalized seedlings infected with VL were transplanted to pots and grown outdoor (open air) where they experienced similar climatic conditions as field grown plants. The duration of this experiment was 12 weeks, until most genotypes reached the fruit development stage (BBCH 80).

\subsubsection{Plant materials and growing conditions}

For this study, forty B. napus genotypes (thirty six lines and four reference varieties) evaluated in the 2011/12 field experiment were used (Appendix 2.2). Surface sterilized seeds (70\% ethanol for two min) of all genotypes were sown in a multipot tray filled with sterile silica sand and soil (1:1). 
Seedlings were grown in a climate chamber with $16 \mathrm{~h}$ light, $20^{\circ} \mathrm{C}$ temperature and $60 \%$ relative humidity. Two weeks after sowing (BBCH12), seedlings were subjected to vernalization by gradual lowering of light duration and temperature conditions to $10 \mathrm{~h}$ and $4^{\circ} \mathrm{C}$, respectively. After 13 weeks of vernalization, seedlings were acclimatized for three days by daily gradual increasing of light duration and temperature conditions up to $16 \mathrm{~h}$ and $20^{\circ} \mathrm{C}$, respectively.

\subsubsection{Inoculation and disease scoring}

Vernalized seedlings were inoculated with mixed spore suspensions of the highly aggressive VL isolates VL40 and VL43. Inoculum preparation and its density adjustment, method and procedure of inoculation and all other procedures were done exactly the same way as in the greenhouse screening experiments (section 2.2.1.3). Control plants were similarly inoculated with sterile water. In order to avoid cross contaminations due to runoff during rain or irrigation, pots with control plants were kept on trays (Fig. 2.2). Measurement and analysis of AUDPC and stunting effects were done as described in section 4.2.7. Net branching and net growth stage values were calculated by subtracting the average values of mock-inoculated plants from that of the respective VL-inoculated treatments.

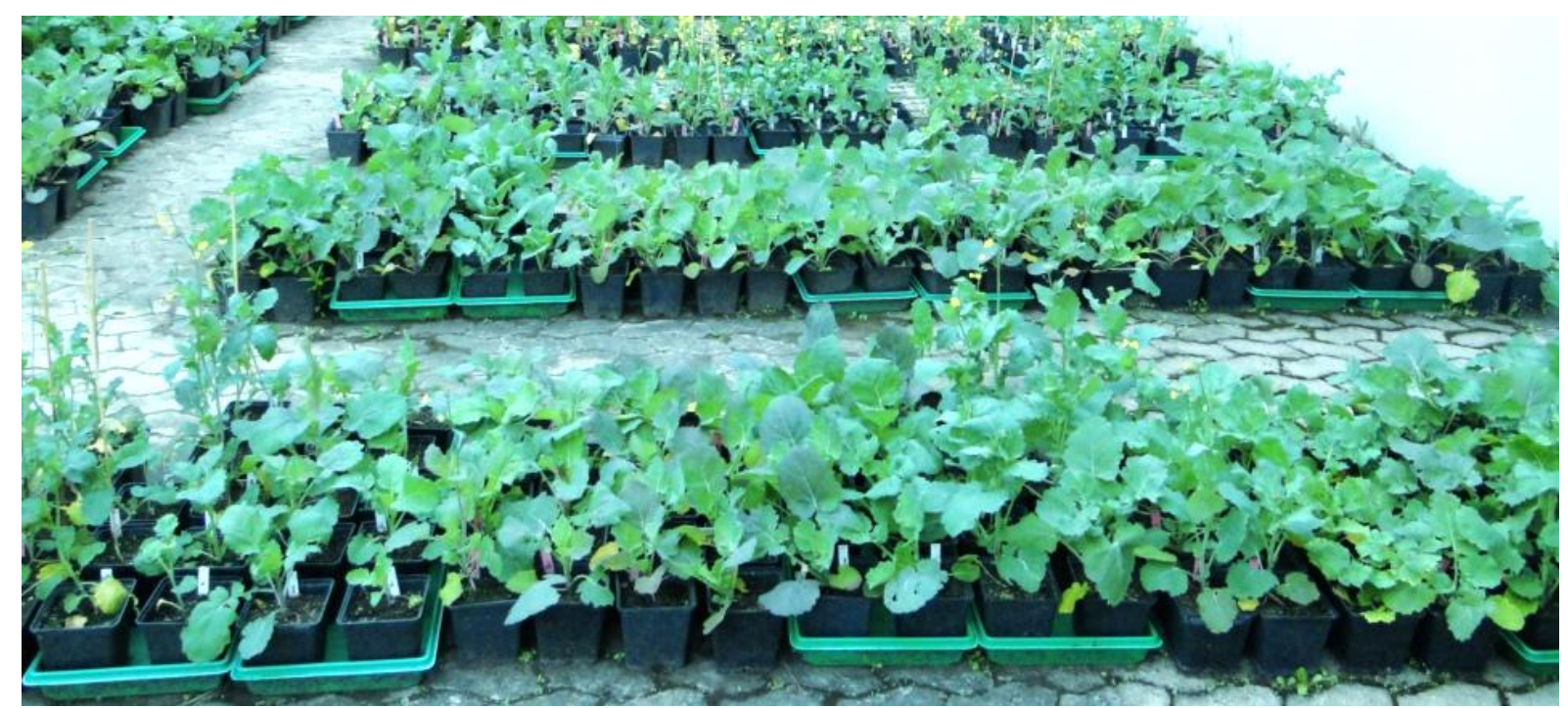

Figure 2.2 Partial view of outdoor experiment: Screening of Brassica napus lines for resistance against Verticillium longisporum. Vernalized plants at $36 \mathrm{DPI}$ are shown. Pot plants placed on trays are mockinoculated plants and those on the surface of the outdoor ground are VL-inoculated treatments.

\subsubsection{Experimental design and outdoor environmental conditions}

The outdoor (open air) pot experiment was conducted at the Department of Plant Pathology and Crop Protection, University of Göttingen. The trial was arranged in a completely randomized design (CRD) with three replications. For each line, 9 VL-inoculated and 9 mock-inoculated plants, each representing three biological replicates (three plants per replication), were used. Immediately after 
inoculation, seedlings were transplanted into 1.5 liter plastic pots filled with river sand and twelve weeks ( $3^{\text {rd }}$ of April 2012 until $26^{\text {th }}$ of July 2012). Average climatic conditions of the outdoor area during the experimental period were $14.2^{\circ} \mathrm{C}$ mean temperature, $74.6 \%$ mean relative humidity and $298.4 \mathrm{~mm}$ total rainfall (Appendix 2.4). All other agronomic practices including fertilization (Hakaphos COMPO, Germany) and insecticide application against flower beetles were applied following standard recommendations. Whenever required, plants were appropriately irrigated with tap water.

\subsubsection{Agronomic and disease data collection}

Disease severity, plant height, number of branches per plant, and phenological growth stage were measured and analyzed according to the procedures described in chapter 4 . Disease index was assessed weekly from 7 to 56 DPI. Number of primary branches per plant in control and infected plants was counted on a weekly basis from 35 to $56 \mathrm{DPI}$. To obtain net branching values, number of branches in mock-inoculated plants of each line was counted and this value was subtracted from corresponding VL-inoculated treatments. Similarly, assessment of phenological stages was assessed from 35 to $56 \mathrm{DPI}$ using the $\mathrm{BBCH}$ scale (Appendix 4.13). Measurement of plant height was done at the end of the experiment (84 DPI).

\subsubsection{Data analysis}

For all lines screened in field experiments, mean data obtained from field, outdoor and greenhouse screening experiments were subjected to one-way ANOVA using the STATISTICA package for windows (version 10, StatSoft, inc. 2011). As a post-hoc comparison among genotypes, Fisher's least significant difference (LSD) test at $\mathrm{P}<0.05$ was applied. For the rest of all data obtained from greenhouse and outdoor experiments, mean values of the different disease parameters derived from each line were compared with the respective values of the reference varieties. To analyze the relationship among the results of greenhouse, outdoor and/or field experiments as well as field results across locations, Pearson's correlation analysis was performed.

\subsection{Results and discussion}

\subsubsection{Greenhouse screening}

For the B. napus - V.longisporum pathosystem, a well-established and efficient greenhouse screening technique that enables screening of large numbers of plants within short period of time is available (Eynck, 2008). Using this method, a total of 235 B. napus lines were screened for VL resistance in four rounds of independent experiments. Disease development was monitored by measuring disease severity and stunting. In general, a wide range of variation in disease reaction among the 
different B. napus lines was observed. There were large numbers of genotypes that showed better performance than the resistant reference variety. Some others had extremely high disease levels even when compared to the susceptible reference control. Figure 2.3 shows the results of some lines screened in different experiments that represent the range of responses from extremely susceptible to highly resistant phenotypes. Detailed information and results of all lines screened in the greenhouse experiments are found in Appendix 2.1 and Appendix 2.3.

According to the results of disease severity assessments, from the total 235 lines screened in all greenhouse experiments, 90 had lower normalized net AUDPC (NNA) values than the mean value of the resistant reference variety Express (0.58). Eight lines showed extremely high levels of NNA which were greater than the values recorded from the susceptible reference variety Falcon (NNA=1.42). The remaining 137 lines had NNA values ranging between the resistant and susceptible reference varieties (Appendix 2.3). Comparable results have also been found from the analysis of relative stunting data. With some exceptions, the extent of stunting due to VL infection closely correlates with net AUDPC values of most lines. That means in most cases genotypes with severe stunting also had high NNA values and vice versa. Nevertheless, some lines with very low NNA were found to be severely stunted.

For instance, from 90 lines with less NNA values than the resistance reference variety Express, 35 had greater normalized relative stunting (NRS) values than the one recorded from the same resistant reference variety (NRS=0.53). Of these lines, six of them,SW08-309035, WVB226, WVB225, WVB227, WVB232 and SYNVER256 (Appendix 2.3), showed extreme stunting with NRS values greater than twice the value recorded in Express (i.e NRS > 1.06). In general, considering plant height as a disease parameter, 73 lines representing 31\% of the total number had lower NRS value than the resistant reference cultivar Express (NRS $=0.53$ ). There were only six lines (SYNVER258, DSV10-42, DSV10-41, KWS204, WVB223, and SYNVER256) that showed more severe stunting compared to the resistant cultivar Falcon (NRS=1.47). The NRS value of the remaining 156 lines representing more than $66 \%$ of the total number lay between the values of the resistant and susceptible controls (Appendix 2.3). This is a more or less similar proportion compared to the one observed in disease severity (NNA) results.

Correlation analysis between NNA and NRS measurements showed a significant, strong and positive correlation (Fig. 2.4) indicating that these parameters are most appropriate and reliable phenotypic traits for evaluation of VL resistance in oilseed rape under controlled conditions. Overall, from the results of the four independent greenhouse experiments, 23 lines (NICK191,NICK189, DSV10-33, NICK186, DSV10-30, NICK190, SW08-309026, DSV10-32, SW08-309029, NICK187, SRG228, NICK184, 


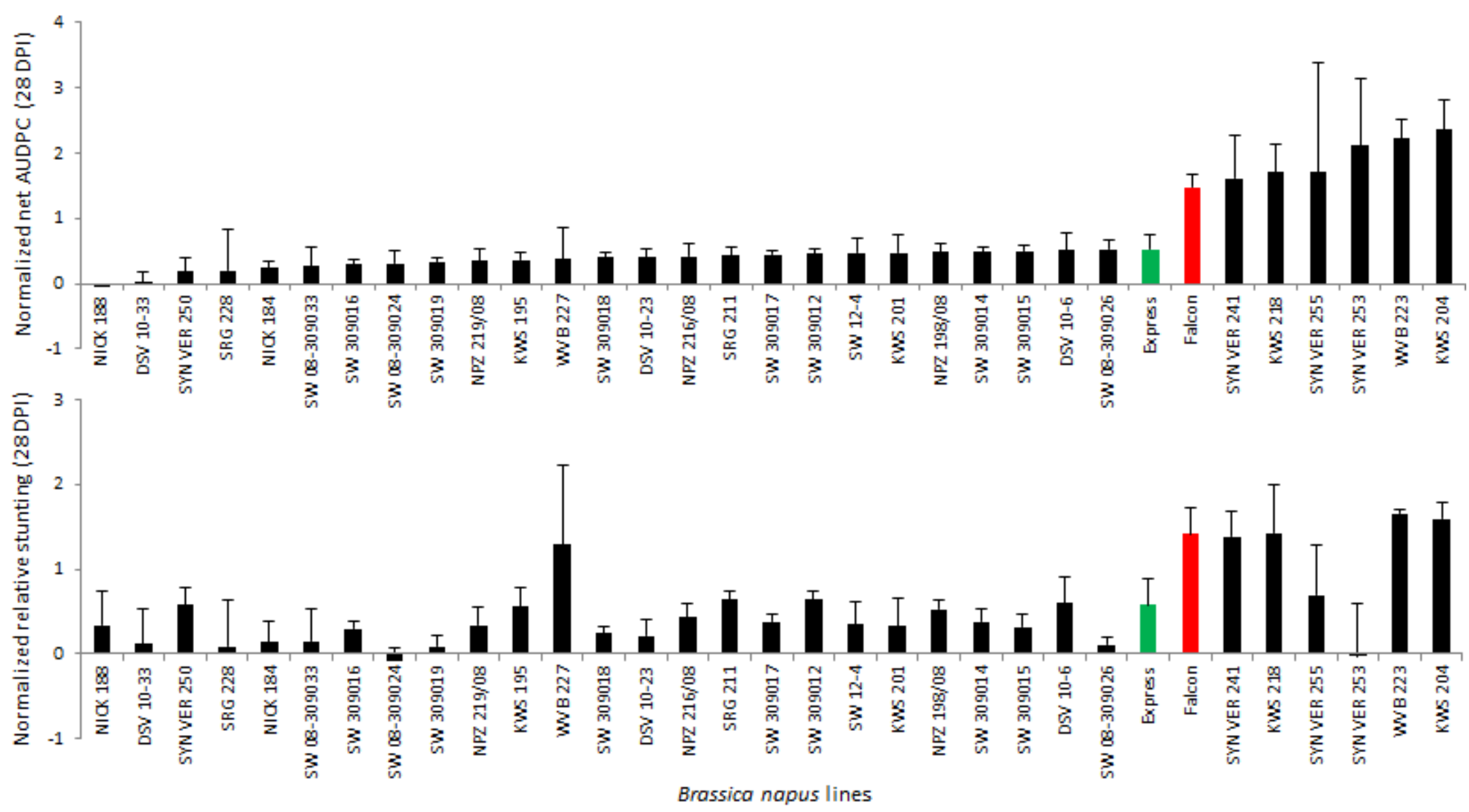

Figure 2.3 Response of Brassica napus lines to Verticillium longisporum infection under greenhouse conditions. Normalized mean net AUDPC (top) and relative stunting (bottom) data of 33 representative Brassica napus lines from four different screenings are shown. For both parameters data shown are the means of 20 plants. Genotypes are sorted according to increasing level of net AUDPC values. Green and red bars show mean values derived from four independent experiments (GHS42, GHS43, GHS44 and GHS46) for the reference variety Express (resistant) and Falcon (susceptible). Errors bars indicate standard deviations. DPI, days after inoculation with Verticillium longisporum. 
WVB231, SW08-309033, NICK192, SW309016, SW08-309024, SW08-309030, DSV10-29, DSV10-28, SW309019, NPZ219/08, and KWS200) that had NNA and NRS values less than 0.40 and showed very strong resistance against VL were identified. These lines are recommended for further test under field condition and to be considered in future breeding works.

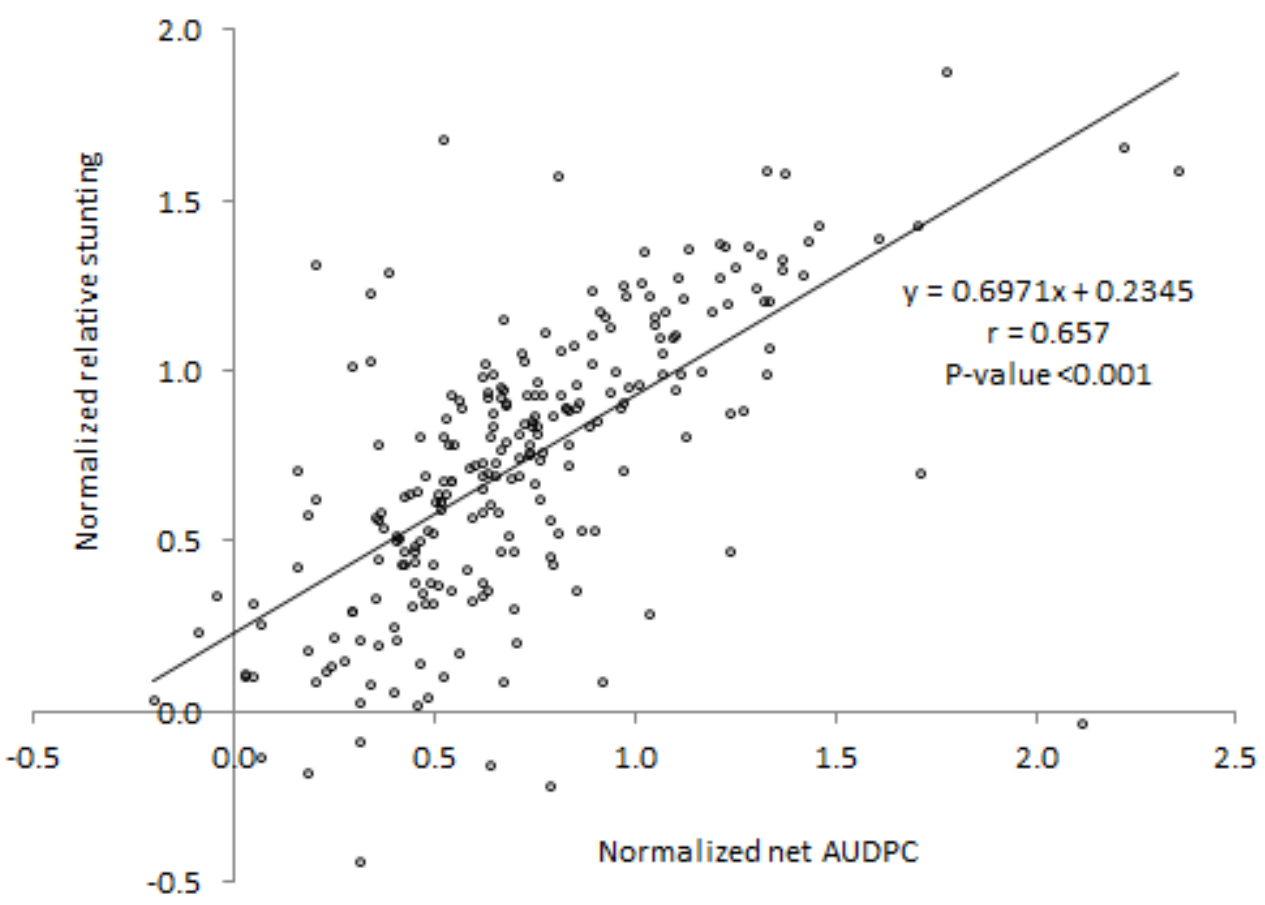

Figure 2.4 Correlation analysis describing the relationship between disease assessment parameters recorded in the greenhouse experiment. Analysis was performed using mean normalized net AUDPC and relative stunting data from 235 Brassica napus lines and two reference varieties $(n=237)$. Pearson correlation analysis was done using STATISTICA (Version 10 StatSoft, inc. 2011).

\subsubsection{Field trials}

To better understand the results of the different field experiments and perform correlation analyses among the results of different locations, years and types of experiments, data analysis for the results of field trials was divided into three parts. In the first part (2.4.2.1), ANOVA and correlation analysis was conducted for $14 \mathrm{DH}$ lines which were tested for three seasons and across all locations. The second part (2.4.2.2) shows the results of 22 lines which were tested only once in the field (i.e. in one season trial only). For both the first and the second part, mean data obtained from phenotypic and molecular disease evaluation of each $B$. napus line was compared with the corresponding values of the resistant and susceptible reference varieties. The third part (2.4.2.3), focused on the molecular detection and quantification of $V$. longisporum in the stems at different growth stages of field grown resistant and susceptible reference OSR varieties. 


\subsubsection{B. napus $\mathrm{DH}$ lines evaluated for $V$. longisporum resistance in multi-locational field trials for three consecutive seasons (years)}

Fourteen best performing $B$. napus lines (13 DH lines and one accession) which were selected based on previous greenhouse screening results were evaluated for three consecutive growing seasons (2010/11, 2011/12 and 2012/13) across three locations differing in climatic and disease conditions (Table 2.2). Source and descriptions of all plant materials can be found in Appendix 2.2. Results of the experiments conducted in Fehmarn, Svalöv and Göttingen are discussed as follows.

Analysis of data obtained from Fehmarn showed that only in a few cases field disease parameters have shown significant differences (Table 2.3). In 2010/11, disease evaluation data was obtained from assessment of disease severity from shoots and roots of stubbles samples. One-way ANOVA showed significant differences in the shoot disease index (SDI) but not in the root disease index (RDI). Interestingly, there were significant differences in SDI between the two susceptible varieties (Falcon and Laser) and the resistant variety Oase. Four lines (SEM05-500256, SW08-190001-7, SW08190002-8 and SW08-190002-9) had also significantly lower SDI than both susceptible controls. Of these three lines SW08-190002-8 was the one that had the lowest SDI which is even significantly lower than the one recorded from both resistant reference varieties. SDI and RDI evaluations in 2011/12 showed no significant differences among all genotypes. From the same experiment, molecular quantification of VL biomass by $\mathrm{GPCR}$, however, revealed significant differences. Considering this parameter, the difference between the resistant and susceptible controls was significant. Accordingly, the amount of VL DNA quantified from the susceptible cultivars Falcon and Laser was 9.8 and $11.4 \mathrm{ng}$ per gram of stem dry matter, respectively. In the resistant varieties Express and Oase, the amount was 0.8 and $1.8 \mathrm{ng} / \mathrm{g}$, respectively. Regarding the DH lines, except SW08190001-11 and SW08-190001-1, all other lines had significantly lower amounts of VL DNA than both susceptible controls. The least amount of VL DNA, which is significantly lower than both resistant controls, was detected from accession SEM05-500256. In 2012/13, again data only on SDI and RDI measurements was available and both measurements showed no significant differences among all genotypes. Unlike field conditions however, analysis of the phenotypic disease evaluation parameters used in outdoor and greenhouse experiments (NNA and NRS) showed significant differences between the reference controls and among the tested genotypes as well (Table 2.3).

When we compare field performance (in Fehmarn) with outdoor and greenhouse screening results, it was found that VL DNA was the only field parameter that showed strong and significant correlations across years (with SDI 2010/11; $r=0.53$ ), with outdoor NNA $(r=0.51)$ and greenhouse NNA ( $r=0.62)$ results. It was also noted that unlike RDI, field results from assessment of SDI were better correlated with field VLDNA as well as with greenhouse and outdoor results. Regarding the 
correlation of SDI and RDI, among growing seasons, there was only one case where a significant correlation was observed (i.e SDI 2011 vs SDI 2012; r=0.50). Of the two parameters used in the outdoor and greenhouse experiments, compared to NRS, NNA was more correlated with field data (Table 2.6A), somehow suggesting an effect of genotype variability in determining the extent of VLinduced stunting. This is in strong agreement with the observations of greenhouse screening experiments where some genotypes with resistant reactions in AUDPC measurements showed severe stunting (Appendix 2.3). According to the results of three years experiments in Fehmarn, compared to the susceptible control reference varieties, except SW08-190002-1 and SW 08-1900025 , the rest 12 lines were found to be promising materials. Of which genotypes SEM05-500256, SW08-190002-8, SW08-190001-25, SW08-190001-7, SW08-190001-12, SW08-190002-9, SW08190001-11 and SW08-190002-11 have shown more or less consistent resistance responses in field experiments in different years, and under outdoor and greenhouse conditions (Fig. 2.3).

Similar to the results in Fehmarn, SDI and RDI data recorded in 2010/11 and 2011/12 experiments in Svalöv showed no significant differences among all genotypes (Table 2.4). In contrary to Fehmarn however, severity of SDI in 2010/11 and RDI in 2010/11 and 2011/12 were relatively lower in Svalöv. Conversely, RDI in 2010/11 was slightly higher in Svalöv than in Fehmarn. Interestingly, quantification of VL DNA from the 2011/12 season experiment in Svalöv also showed significant differences among genotypes. Unlike in Fehmarn, however, even though up to twofold VL DNA was quantified from susceptible varieties compared to resistant controls, this difference was not statistically significant. This could be because of the high variability between replicates. Regarding the different lines, seven (SEM05-500256, SW08-190001-12, SW08-190002-5, SW08-190001-11, SW08-190002-16, SW08-190001-1 and SW08-190002-9) had significantly lower amounts of VL DNA compared to the susceptible controls. Of these lines, three of them (SEM05-500256, SW08-19000112 and SW08-190002-5) had significantly lower VL DNA than both resistant controls. Assessment of disease severity by qPCR in 2011/12 also revealed the presence of a relatively higher disease infestation (for most genotypes) in Svalöv than in Fehmarn. Since no sufficient numbers of plants remained at harvest due to loss of most plants by the hard winter in Svalöv, no data was available from 2012/13 (Table 2.4).

According to the correlation analysis results between field performance in Svalöv vs outdoor and greenhouse, the only significant correlation was found between VL DNA and greenhouse NRS $(r=0.56)$.The other significant correlations were found between SDI and RDI in 2010/11 ( $r=0.73$ ) and 2011/12 ( $r=0.91$ ) (Table 2.6B). In general, it was noticed that compared to Fehmarn, results obtained from field experiments in Svalöv were poorly correlated with each other and with greenhouse and outdoor results. For instance in Fehmarn there was significant correlation $(r=0.50)$ between the 
2010/11 and 2011/12 growing seasons in SDI and RDI measurements (Table 2.6A) which was not the case in Svalöv (Table 2.6B). Furthermore, few field parameters from Fehmarn (RDI and VLDNA) at least showed a significant correlation with the outdoor NNA results (Table 2.6A), but none of the parameters from Svalöv had a significant correlation with the outdoor results (Table 2.6B). Apart from climatic and soil factors, the variation in compositions of different VL isolates in the soil and the amount of natural inoculum present in the soil might be among the possible explanations for the differences found across locations. Nevertheless, considering the results of three years of experiments in Svalöv, it was found that genotypes SEM05-500256, SW08-190001-12, SW08190002-5, SW08-190001-7 and SW08-190001-25 were the top performing lines (Table 2.4) showing consistent resistance both under field and controlled experimental conditions.

In Göttingen, the trials in 2010/11 and 2011/12 were destroyed due to severe winter. As a result, data from this site was only available from 2012/13 (Table 2.5). Due to low/no natural disease infestation, the experiment at this location was conducted with additional application of inoculum (see 2.2.2.4). However, even after application of additional inoculum, level of disease severity as evidenced by phenotypic and molecular parameters was significantly lower as compared to the other two locations. Regarding SDI and RDI, with the exception of Oase and SW 08-190002-8 that had significantly lower level of disease compared to the susceptible check Laser, no significant difference was found among the rest of the $\mathrm{DH}$ lines and the reference varieties. The overall disease severity level in all genotypes including the susceptible controls was very low, with an average disease index less than 2.0, referring to slight infestation (Fig. 2.1).

With regard to the qPCR data however, a better differentiation of the different genotypes was achieved. More importantly, significant differences were found between the resistant and susceptible reference varieties. The highest amount of VL DNA was quantified in the two susceptible reference varieties and the $\mathrm{DH}$ line SW08-190001-11. On the other hand, compared to both susceptible controls, lines SW08-190001-25, SEM05-500256, SW08-190001-7, SW08-190002-18, SW08-190002-1 and the two resistant varieties had significantly lower fungal biomass (Table 2.5). As in the other two locations, compared to the phenotypic disease assessment, field evaluation of VL resistance via qPCR showed very strong and significant correlations with field SDI ( $r=0.85)$, field RDI $(r=0.68)$ and greenhouse AUDPC $(r=0.55)$ recordings. Furthermore, significant correlation of RDI with SDI ( $r=0.83)$ and greenhouse NRS ( $r=0.56)$ was found (Table 2.6C). Considering a single season field result of this location, it was found that lines SW08-190001-25, SEM05-500256, SW08-1900017, SW08-190002-9, SW08-190001-12, sW08-190001-1, sW08-190002-8, SW08-190002-16 and 
SW08-190002-11 (Table 2.5) have shown a reasonably consistent and better performance in field, greenhouse and outdoor conditions.

To study the overall relationships in field performance of $B$. napus lines across the three locations, mean phenotypic and molecular disease data obtained from 18 genotypes (14 lines and 4 reference varieties) were subjected to Pearson's correlation analysis. From the 2010/11 and 2011/12 trials, since no data was available from Göttingen, correlation between results of Fehmarn and Svalöv locations was analyzed. Unfortunately, none of the disease parameters recorded in 2010/11, 2011/12 and 2012/213 showed any significant correlation between locations. Interestingly, similar to the correlations across years (Table 2.6), results from quantification of VL DNA in the 2011/12 trial from Svalöv showed a significant positive correlation ( $r=0.86)$ with the same parameter in Fehmarn. In 2012/13, no data was available from Svalöv and correlation analysis was performed for Göttingen and Fehmarn sites only. Here, none of the parameters showed any significant correlations (Table 2.7). Local differences in terms of climatic and soil factors and diversity of VL isolates that possibly determine host-pathogen interactions might be the possible reasons for the poor correlation of results across the different locations.

In conclusion, among the 14 B. napus lines evaluated for three years (seasons) in multiple locations, SEM05-500256, SW08-190001-12, SW08-190001-7, SW08-190001-25, SW08-190002-9, SW08190002-8 and SW08-190002-11, that showed consistently higher resistance in greenhouse, outdoor and field trials in at least two locations, are highly recommended for use as parent materials in future breeding aiming at integration of $V$. longisporum resistance traits into commercial winter oilseed rape varieties. 
Table 2.3 Response of Brassica napus double haploid lines to Verticillium longisporum infection under field conditions in Fehmarn. Comparisons of field performance across years and between field and greenhouse/outdoor screening results of 14 lines and 4 reference varieties screened during all season trials are shown.

\begin{tabular}{|c|c|c|c|c|c|c|c|c|c|c|c|}
\hline \multirow{3}{*}{ Genotype code } & \multicolumn{7}{|c|}{ Field trial (Fehmarn) } & \multirow{2}{*}{\multicolumn{2}{|c|}{ Outdoor }} & \multirow{2}{*}{\multicolumn{2}{|c|}{ Greenhouse }} \\
\hline & \multicolumn{2}{|c|}{$2010 / 11$} & \multicolumn{3}{|c|}{$2011 / 12$} & \multicolumn{2}{|c|}{$2012 / 13$} & & & & \\
\hline & SDI & $\mathrm{RDI}^{\mathrm{ns}}$ & $S D I^{\mathrm{ns}}$ & $\mathrm{RDI}^{\mathrm{ns}}$ & VLDNA & $\mathrm{SDI} I^{\mathrm{ns}}$ & $\mathrm{RDI}^{\mathrm{ns}}$ & NNA & NRS & NNA & NRS \\
\hline SEM 05-500256 & $2.4 \pm 0.1^{c d^{-}}$ & $1.8 \pm 0.1$ & $2.1 \pm 0.1$ & $2.3 \pm 0.4$ & $0.13 \pm 0.1^{f}$ & $1.41 \pm 0.3$ & $1.32 \pm 0.5$ & $0.8+0.2^{\text {defg }}$ & $0.8+0.4^{\text {cde }}$ & $-0.02 \pm 0.1^{f}$ & $0.20 \pm 0.3^{\mathrm{d}}$ \\
\hline SW 08-190002-8 & $1.8+0.1^{\mathrm{e}}$ & $1.4+0.1$ & $1.7 \pm 0.3$ & $1.7 \pm 0.2$ & $0.17 \pm 0.0^{\text {ef }}$ & $1.93+0.8$ & $2.12 \pm 1.2$ & $0.7+0.5^{\mathrm{efg}}$ & $0.6+0.3^{\text {def }}$ & $1.06 \pm 0.1^{\mathrm{abcd}}$ & $0.54+0.4^{\mathrm{bcd}}$ \\
\hline SW 08-190001-25 & $2.5 \pm 0.3^{\mathrm{bcd}}$ & $1.6 \pm 0.2$ & $1.6 \pm 0.3$ & $1.5 \pm 0.3$ & $0.32 \pm 0.4^{\text {def }}$ & $2.17 \pm 0.5$ & $1.92 \pm 0.1$ & $0.3+0.3^{g h}$ & $0.0 \pm 0.9^{\text {ef }}$ & $0.95 \pm 0.4^{\mathrm{bcd}}$ & $0.28 \pm 0.6^{d}$ \\
\hline SW 08-190002-5 & $3.4+0.1^{\mathrm{a}}$ & $1.9 \pm 0.1$ & $2.4 \pm 0.2$ & $2.1 \pm 0.2$ & $0.48 \pm 0.3^{\text {cdef }}$ & $2.04 \pm 0.1$ & $2.16 \pm 0.2$ & $1.1+0.5^{\text {bcdef }}$ & $0.2+0.0^{\text {ef }}$ & $1.03 \pm 0.4^{\mathrm{bcd}}$ & $0.63 \pm 0.6^{\mathrm{abcd}}$ \\
\hline SW 08-190001-7 & $2.3 \pm 0.4^{\mathrm{de}}$ & $1.6 \pm 0.1$ & $2.0 \pm 0.6$ & $2.0 \pm 0.4$ & $0.54 \pm 0.3^{\text {bcdef }}$ & $1.86 \pm 0.2$ & $1.71 \pm 0.4$ & $1.1 \pm 0.3^{\mathrm{bcde}}$ & $0.7 \pm 0.9^{\text {cde }}$ & $0.96 \pm 0.5^{\mathrm{bcd}}$ & $0.31 \pm 0.3^{\mathrm{cd}}$ \\
\hline SW 08-190001-12 & $2.7 \pm 0.2^{\mathrm{bcd}}$ & $1.7 \pm 0.2$ & $2.5 \pm 0.0$ & $2.4 \pm 0.1$ & $0.69 \pm 0.8^{\text {cdef }}$ & $2.52 \pm 0.4$ & $3.24 \pm 1.1$ & $1.0 \pm 0.1^{\text {cdef }}$ & $0.5+0.3^{\text {def }}$ & $0.82 \pm 0.0^{\mathrm{cd}}$ & $0.55 \pm 0.4^{b c d}$ \\
\hline SW 08-190002-9 & $2.3 \pm 0.3^{\mathrm{de}}$ & $1.4 \pm 0.2$ & $2.1 \pm 0.5$ & $2.2 \pm 0.5$ & $0.72 \pm 0.4^{\text {bcdef }}$ & $1.26 \pm 0.2$ & $1.38 \pm 0.5$ & $0.0+0.1^{\mathrm{hi}}$ & $2.2 \pm 1.6^{\mathrm{b}}$ & $0.94 \pm 0.1^{\mathrm{bcd}}$ & $0.60 \pm 0.4^{b c d}$ \\
\hline Express $^{(\mathrm{RC})}$ & $2.9 \pm 0.4^{\mathrm{abc}}$ & $1.8 \pm 0.1$ & $2.3 \pm 0.0$ & $2.2 \pm 0.1$ & $0.81 \pm 0.5^{\text {bcde }}$ & $1.79 \pm 0.5$ & $1.90 \pm 0.1$ & $0.3+0.4^{g h}$ & $0.3+0.6^{\text {ef }}$ & $0.69 \pm 0.3^{\text {de }}$ & $0.86 \pm 0.2^{\mathrm{abcd}}$ \\
\hline SW 08-190001-6 & $3.0 \pm 0.3^{\mathrm{abc}}$ & $1.6 \pm 0.1$ & $2.1 \pm 0.1$ & $2.3 \pm 0.1$ & $0.98 \pm 1.0^{\text {bcdef }}$ & $1.73 \pm 0.3$ & $2.04 \pm 0.1$ & $1.4+0.1^{b c d}$ & $3.6 \pm 0.4^{a}$ & $1.27 \pm 0.1^{\mathrm{ab}}$ & $0.72 \pm 0.4^{\mathrm{abcd}}$ \\
\hline SW 08-190002-1 & $2.8 \pm 0.5^{\mathrm{bcd}}$ & $1.9 \pm 0.2$ & $1.8 \pm 0.5$ & $1.9+0.6$ & $1.14 \pm 1.2^{\text {bcde }}$ & $2.57 \pm 1.0$ & $2.36 \pm 0.9$ & $1.6 \pm 0.2^{\mathrm{b}}$ & $1.5 \pm 0.9^{\mathrm{bcd}}$ & $1.18 \pm 0.5^{\mathrm{abc}}$ & $1.08 \pm 0.4^{\mathrm{ab}}$ \\
\hline SW 08-190002-18 & $2.7 \pm 0.1^{\mathrm{bcd}}$ & $1.7 \pm 0.3$ & $2.2 \pm 0.4$ & $2.0 \pm 0.5$ & $1.20 \pm 0.8^{\text {bcde }}$ & $2.06 \pm 0.1$ & $2.30 \pm 0.4$ & $1.4 \pm 0.3^{b c}$ & $1.7 \pm 0.3^{\mathrm{bc}}$ & $1.14 \pm 0.5^{\mathrm{abcd}}$ & $0.78 \pm 0.7^{\mathrm{abcd}}$ \\
\hline SW 08-190002-16 & $3.0 \pm 0.3^{\mathrm{abc}}$ & $1.6 \pm 0.1$ & $2.4 \pm 0.2$ & $2.3 \pm 0.1$ & $1.21 \pm 1.1^{\text {bcde }}$ & $2.35 \pm 0.6$ & $3.02 \pm 1.4$ & $0.4 \pm 0.2^{\mathrm{fgh}}$ & $-1.3 \pm 0.5^{g}$ & $1.19 \pm 0.2^{\mathrm{abc}}$ & $0.58 \pm 0.6^{\mathrm{bcd}}$ \\
\hline SW 08-190002-11 & $2.9 \pm 0.1^{\mathrm{abc}}$ & $1.6 \pm 0.0$ & $2.2 \pm 0.3$ & $2.3 \pm 0.3$ & $1.33 \pm 0.5^{b c}$ & $1.87 \pm 0.5$ & $2.52 \pm 0.7$ & $0.7 \pm 0.4^{\text {efg }}$ & $-0.5 \pm 0.1^{\mathrm{g}}$ & $1.19 \pm 0.2^{\mathrm{abc}}$ & $0.77 \pm 0.4^{\mathrm{abcd}}$ \\
\hline Oase ${ }^{(R C)}$ & $2.8 \pm 0.4^{b c d}$ & $1.7 \pm 0.3$ & $2.2 \pm 0.2$ & $2.3 \pm 0.4$ & $1.75 \pm 1.2^{\mathrm{bcd}}$ & $1.34 \pm 0.4$ & $1.40 \pm 0.6$ & $0.0 \pm 0.2^{\mathrm{hi}}$ & $1.0 \pm 0.4^{\text {cde }}$ & $0.36 \pm 0.1^{\text {ef }}$ & $0.42 \pm 0.2^{\mathrm{bcd}}$ \\
\hline SW 08-190001-11 & $2.6 \pm 0.1^{b c d}$ & $1.5 \pm 0.1$ & $2.4 \pm 0.1$ & $2.4 \pm 0.1$ & $2.09 \pm 0.2^{\mathrm{abc}}$ & $1.98 \pm 0.3$ & $1.94 \pm 0.1$ & $-0.4+0.0^{i}$ & $0.9 \pm 0.5^{\text {cde }}$ & $1.18 \pm 0.2^{\mathrm{abc}}$ & $0.99 \pm 0.1^{\mathrm{abc}}$ \\
\hline SW 08-190001-1 & $2.8 \pm 0.5^{\mathrm{bcd}}$ & $1.8 \pm 0.1$ & $2.5 \pm 0.2$ & $2.3 \pm 0.0$ & $2.95 \pm 1.4^{\mathrm{ab}}$ & $2.16 \pm 0.3$ & $2.56 \pm 0.6$ & $0.8 \pm 0.3^{\text {efg }}$ & $1.1 \pm 0.7^{\text {cde }}$ & $0.97 \pm 0.2^{\mathrm{bcd}}$ & $0.33 \pm 0.6^{\mathrm{cd}}$ \\
\hline Falcon $^{(\mathrm{SC})}$ & $3.1 \pm 0.0^{\mathrm{ab}}$ & $1.8 \pm 0.1$ & $2.3 \pm 1.0$ & $2.3 \pm 1.2$ & $9.77 \pm 5.7^{\mathrm{a}}$ & $2.18 \pm 0.5$ & $2.68 \pm 0.5$ & $1.3 \pm 1.1^{\text {bcde }}$ & $0.3 \pm 0.5^{\mathrm{ef}}$ & $1.49 \pm 0.1^{\mathrm{a}}$ & $1.32 \pm 0.2^{\mathrm{a}}$ \\
\hline Laser $^{(\mathrm{SC})}$ & $3.4 \pm 0.1^{\mathrm{a}}$ & $1.8 \pm 0.1$ & $2.1 \pm 0.4$ & $2.0 \pm 0.4$ & $11.4 \pm 6.6^{\mathrm{a}}$ & $1.98 \pm 0.1$ & $1.96 \pm 0.1$ & $2.4 \pm 0.1^{a}$ & $2.4 \pm 0.9^{b}$ & $1.05 \pm 0.1^{\mathrm{abcd}}$ & $1.06 \pm 0.1^{\mathrm{ab}}$ \\
\hline
\end{tabular}

SDI, shoot disease index. RDI, root disease index. NNA, net normalized AUDPC. NRS, normalized relative stunting. VLDNA, Verticillium longisporum DNA (ng/g) in hypocotyl at BBCH80. (RC), resistant control. (SC), susceptible control. *, not a double haploid line. For all parameters mean \pm standard deviation data is shown. Greenhouse results are means of 60 plants derived from three independent experiments. In outdoor experiment, vernalized seedlings inoculated with VL were transplanted to pots and grown outdoor (open air condition). Genotypes are sorted according to field VLDNA quantification results that showed better correlation with other field, outdoor and GH parameters and across locations. For each parameter, means followed by the same letter are not significantly different at $\mathrm{p}=0.05$. Mean values under the parameters indicated by the superscript ' $n s$ ' are not significantly different at $p=0.05$. 
Table 2.4 Response of Brassica napus double haploid lines to Verticillium longisporum infection under field conditions in Svalöv. Comparisons of field performance across years and between field and greenhouse/outdoor screening results of 14 lines and 4 reference varieties screened during all season trials are shown.

\begin{tabular}{|c|c|c|c|c|c|c|c|c|c|c|}
\hline \multirow{3}{*}{ Genotype code } & \multicolumn{6}{|c|}{ Field trial (Svalöv) } & \multirow{2}{*}{\multicolumn{2}{|c|}{ Outdoor }} & \multirow{2}{*}{\multicolumn{2}{|c|}{ Greenhouse }} \\
\hline & \multicolumn{2}{|c|}{ 2010/11 } & \multicolumn{3}{|c|}{ 2011/12 } & \multirow{2}{*}{$\begin{array}{c}2012 / 13 \\
\text { (ND) }\end{array}$} & & & & \\
\hline & $S D I^{\text {ns }}$ & $\mathrm{RDI}^{\mathrm{ns}}$ & $S D I^{\text {ns }}$ & $\mathrm{RDI}^{\mathrm{ns}}$ & VLDNA & & NNA & NRS & NNA & NRS \\
\hline SEM 05-500256 & $2.0 \pm 0.2$ & $2.1 \pm 0.1$ & $1.2 \pm 0.1$ & $1.3 \pm 0.1$ & $0.26 \pm 0.1^{\mathrm{ef}}$ & - & $0.8 \pm 0.2^{\text {defg }}$ & $0.8+0.4^{\text {cde }}$ & $-0.02 \pm 0.1^{f-}$ & $0.20 \pm 0.3^{\mathrm{d}}$ \\
\hline SW 08-190001-12 & $2.6 \pm 0.7$ & $2.8 \pm 0.8$ & $1.4 \pm 0.2$ & $1.5 \pm 0.2$ & $0.34 \pm 0.2^{\mathrm{ef}}$ & - & $1.0 \pm 0.1^{\text {cdef }}$ & $0.5 \pm 0.3^{\operatorname{def}}$ & $0.82 \pm 0.0^{c d}$ & $0.55 \pm 0.4^{\mathrm{bcd}}$ \\
\hline SW 08-190002-5 & $1.3 \pm 0.2$ & $2.3 \pm 0.8$ & $1.2 \pm 0.2$ & $1.3 \pm 0.2$ & $0.43 \pm 0.6^{f}$ & - & $1.1 \pm 0.5^{\text {bcdef }}$ & $0.2 \pm 0.0^{\mathrm{ef}}$ & $1.03 \pm 0.4^{\mathrm{bcd}}$ & $0.63 \pm 0.6^{\mathrm{abcd}}$ \\
\hline SW 08-190001-11 & $2.7 \pm 0.4$ & $2.4 \pm 0.6$ & $2.0 \pm 0.5$ & $2.0 \pm 0.4$ & $0.47 \pm 0.4^{\text {def }}$ & - & $-0.4 \pm 0.0^{i}$ & $0.9 \pm 0.5^{\text {cde }}$ & $1.18 \pm 0.2^{\mathrm{abc}}$ & $0.99 \pm 0.1^{\mathrm{abc}}$ \\
\hline SW 08-190002-16 & $2.7 \pm 1.0$ & $2.9 \pm 0.8$ & $1.6 \pm 0.1$ & $1.6 \pm 0.2$ & $0.67 \pm 0.1^{\text {cdef }}$ & - & $0.4 \pm 0.2^{\mathrm{fgh}}$ & $-1.3 \pm 0.5^{g}$ & $1.19 \pm 0.2^{\mathrm{abc}}$ & $0.58 \pm 0.6^{b c d}$ \\
\hline SW 08-190001-1 & $2.8 \pm 0.7$ & $2.5 \pm 0.3$ & $1.4 \pm 0.3$ & $1.5 \pm 0.4$ & $0.96 \pm 0.0^{\text {cdef }}$ & - & $0.8 \pm 0.3^{\mathrm{efg}}$ & $1.1 \pm 0.7^{\text {cde }}$ & $0.97 \pm 0.2^{\mathrm{bcd}}$ & $0.33 \pm 0.6^{c d}$ \\
\hline SW 08-190002-9 & $3.0 \pm 0.1$ & $2.7 \pm 0.2$ & $1.4 \pm 0.1$ & $1.4 \pm 0.2$ & $0.97 \pm 1.0^{\text {cdef }}$ & - & $0.0 \pm 0.1^{\mathrm{hi}}$ & $2.2 \pm 1.6^{b}$ & $0.94 \pm 0.1^{\mathrm{bcd}}$ & $0.60 \pm 0.4^{\mathrm{bcd}}$ \\
\hline SW 08-190002-1 & $2.5 \pm 0.2$ & $2.0 \pm 0.4$ & $1.1 \pm 0.1$ & $1.2 \pm 0.1$ & $1.05 \pm 0.5^{\text {bcde }}$ & - & $1.6 \pm 0.2^{b}$ & $1.5 \pm 0.9^{\mathrm{bcd}}$ & $1.18 \pm 0.5^{\mathrm{abc}}$ & $1.08 \pm 0.4^{\mathrm{ab}}$ \\
\hline SW 08-190001-6 & $2.5 \pm 1.0$ & $2.4 \pm 0.4$ & $1.6 \pm 0.2$ & $1.7 \pm 0.1$ & $1.45 \pm 0.7^{\text {abcde }}$ & - & $1.4 \pm 0.1^{\mathrm{bcd}}$ & $3.6 \pm 0.4^{\mathrm{a}}$ & $1.27 \pm 0.1^{\mathrm{ab}}$ & $0.72 \pm 0.4^{\mathrm{abcd}}$ \\
\hline SW 08-190001-7 & $2.0 \pm 1.3$ & $1.8 \pm 1.0$ & $1.6 \pm 0.1$ & $1.7 \pm 0.2$ & $1.47 \pm 0.6^{\text {abcde }}$ & - & $1.1 \pm 0.3^{\text {bcde }}$ & $0.7 \pm 0.9^{\text {cde }}$ & $0.96 \pm 0.5^{\mathrm{bcd}}$ & $0.31 \pm 0.3^{\text {cd }}$ \\
\hline SW 08-190002-8 & $2.6 \pm 0.4$ & $2.7 \pm 0.1$ & $1.0 \pm 0.0$ & $1.0 \pm 0.0$ & $2.10 \pm 2.6^{\text {bcde }}$ & - & $0.7 \pm 0.5^{\mathrm{efg}}$ & $0.6 \pm 0.3^{\text {def }}$ & $1.06 \pm 0.1^{\mathrm{abcd}}$ & $0.54 \pm 0.4^{b c d}$ \\
\hline SW 08-190002-18 & $2.9 \pm 0.3$ & $2.8 \pm 0.0$ & $1.8 \pm 0.3$ & $1.9 \pm 0.3$ & $2.14 \pm 2.5^{\mathrm{abcde}}$ & - & $1.4 \pm 0.3^{b c}$ & $1.7 \pm 0.3^{b c}$ & $1.14 \pm 0.5^{\mathrm{abcd}}$ & $0.78 \pm 0.7^{\mathrm{abcd}}$ \\
\hline SW 08-190001-25 & $2.4 \pm 0.2$ & $2.3 \pm 0.2$ & $1.4 \pm 0.2$ & $1.4 \pm 0.2$ & $2.95 \pm 1.4^{\mathrm{abcd}}$ & - & $0.3 \pm 0.3^{g h}$ & $0.0 \pm 0.9^{\text {ef }}$ & $0.95 \pm 0.4^{b c d}$ & $0.28 \pm 0.6^{d}$ \\
\hline Oase $e^{(\mathrm{RC})}$ & $2.9 \pm 0.8$ & $2.9 \pm 0.6$ & $1.3 \pm 0.2$ & $1.2 \pm 0.6$ & $3.27 \pm 3.2^{\mathrm{abcd}}$ & - & $0.0 \pm 0.2^{\mathrm{hi}}$ & $1.0 \pm 0.4^{\text {cde }}$ & $0.36 \pm 0.1^{\mathrm{ef}}$ & $0.42 \pm 0.2^{b c d}$ \\
\hline Express $^{(\mathrm{RC})}$ & $1.3 \pm 0.2$ & $1.6 \pm 0.1$ & $1.4 \pm 0.4$ & $1.8 \pm 0.6$ & $3.89 \pm 1.6^{\mathrm{abc}}$ & - & $0.3 \pm 0.4^{g h}$ & $0.3 \pm 0.6^{\mathrm{ef}}$ & $0.69 \pm 0.3^{\text {de }}$ & $0.86 \pm 0.2^{\mathrm{abcd}}$ \\
\hline SW 08-190002-11 & $2.8 \pm 0.2$ & $2.7 \pm 0.1$ & $1.4 \pm 0.1$ & $1.6 \pm 0.3$ & $3.93 \pm 1.9^{\mathrm{abc}}$ & - & $0.7 \pm 0.4^{\mathrm{efg}}$ & $-0.5 \pm 0.1^{\mathrm{g}}$ & $1.19 \pm 0.2^{\mathrm{abc}}$ & $0.77 \pm 0.4^{\mathrm{abcd}}$ \\
\hline Falcon $^{(\mathrm{SC})}$ & $2.8 \pm 0.5$ & $2.3 \pm 0.2$ & $1.5 \pm 0.5$ & $1.7 \pm 0.5$ & $7.97 \pm 1.8^{\mathrm{ab}}$ & - & $1.3 \pm 1.1^{\text {bcde }}$ & $0.3 \pm 0.5^{\mathrm{ef}}$ & $1.49 \pm 0.1^{\mathrm{a}}$ & $1.32 \pm 0.2^{\mathrm{a}}$ \\
\hline Laser $^{(\mathrm{SC})}$ & $2.5 \pm 1.0$ & $2.4 \pm 1.0$ & $1.7 \pm 0.2$ & $1.7 \pm 0.3$ & $8.82 \pm 0.9^{\mathrm{a}}$ & - & $2.4 \pm 0.1^{\mathrm{a}}$ & $2.4 \pm 0.9^{b}$ & $1.05 \pm 0.1^{\mathrm{abcd}}$ & $1.06 \pm 0.1^{\mathrm{ab}}$ \\
\hline
\end{tabular}

DI, shoot disease index. RDI, root disease index. NNA, net normalized AUDPC. NRS, normalized relative stunting. VLDNA, Verticillium longisporum DNA (ng/g) in hypocotyl at BBCH80. ND, no data due to loss of plants by hard winter. (RC), resistant control. (SC), susceptible control. *, not a double haploid line. For all parameters mean \pm standard deviation data is shown. Greenhouse results are mean of 60 plants derived from three independent experiments. In outdoor experiment, vernalized seedlings inoculated with VL were transplanted to pots and grown outdoor (open-air condition). Genotypes are sorted according to field VLDNA quantification results that showed better correlation with other field, outdoor and GH parameters and across locations. For each parameter, means followed by the same letter are not significantly different at $p=0.05$. Mean values under the parameters indicated by the superscript 'ns' are not significantly different at $p=0.05$. 
Table 2.5 Response of Brassica napus double haploid lines to Verticillium longisporum infection under field conditions in Göttingen. Comparisons of field performance across years and between field and greenhouse/outdoor screening results of 14 lines and 4 reference varieties screened during all season trials are shown.

\begin{tabular}{|c|c|c|c|c|c|c|c|c|c|}
\hline \multirow{3}{*}{ Genotype code } & \multicolumn{5}{|c|}{ Field trial (Göttingen) } & \multirow{2}{*}{\multicolumn{2}{|c|}{ Outdoor }} & \multirow{2}{*}{\multicolumn{2}{|c|}{ Greenhouse }} \\
\hline & 2010/11 & $2011 / 12$ & & $2012 / 13$ & & & & & \\
\hline & (ND) & (ND) & SDI & RDI & VLDNA & NNA & NRS & NNA & NRS \\
\hline Express $^{(\mathrm{RC})}$ & - & - & $1.25+0.2^{\mathrm{ab}}$ & $1.17 \pm 0.1^{\mathrm{ab}}$ & $0.08+0.08^{c}$ & $0.3+0.4^{\mathrm{gh}}$ & $0.3+0.6^{\mathrm{ef}}$ & $0.69+0.3^{\mathrm{de}}$ & $0.86+0.2^{\mathrm{a} b c \mathrm{~d}}$ \\
\hline SW 08-190001-25 & - & - & $1.07 \pm 0.1^{\mathrm{ab}}$ & $1.10 \pm 0.1^{\mathrm{ab}}$ & $0.09+0.06^{c}$ & $0.3+0.3^{g h}$ & $0.0+0.9^{\text {ef }}$ & $0.95 \pm 0.4^{b c d}$ & $0.28 \pm 0.6^{d}$ \\
\hline SEM 05-500256 ${ }^{*}$ & - & - & $1.20 \pm 0.2^{\mathrm{ab}}$ & $1.15 \pm 0.2^{\mathrm{ab}}$ & $0.12+0.13^{c}$ & $0.8+0.2^{\text {defg }}$ & $0.8+0.4^{\text {cde }}$ & $-0.02 \pm 0.1^{f}$ & $0.20 \pm 0.3^{d}$ \\
\hline SW 08-190001-7 & - & - & $1.15 \pm 0.1^{\mathrm{ab}}$ & $1.12 \pm 0.1^{\mathrm{ab}}$ & $0.14 \pm 0.12^{c}$ & $1.1 \pm 0.3^{\text {bcde }}$ & $0.7 \pm 0.9^{\text {cde }}$ & $0.96 \pm 0.5^{\mathrm{bcd}}$ & $0.31 \pm 0.3^{\mathrm{cd}}$ \\
\hline SW 08-190002-18 & - & - & $1.12 \pm 0.0^{\mathrm{ab}}$ & $1.10 \pm 0.0^{\mathrm{ab}}$ & $0.16 \pm 0.12^{c}$ & $1.4 \pm 0.3^{b c}$ & $1.7 \pm 0.3^{b c}$ & $1.14 \pm 0.5^{\mathrm{abcd}}$ & $0.78 \pm 0.7^{\mathrm{abcd}}$ \\
\hline SW 08-190002-1 & - & - & $1.03 \pm 0.1^{\mathrm{ab}}$ & $1.07 \pm 0.1^{\mathrm{ab}}$ & $0.17 \pm 0.13^{c}$ & $1.6+0.2^{b}$ & $1.5 \pm 0.9^{\mathrm{bcd}}$ & $1.18 \pm 0.5^{\mathrm{abc}}$ & $1.08 \pm 0.4^{\mathrm{ab}}$ \\
\hline SW 08-190002-9 & - & - & $1.10 \pm 0.1^{\mathrm{ab}}$ & $1.21 \pm 0.2^{\mathrm{ab}}$ & $0.19+0.14^{c}$ & $0.0 \pm 0.1^{\mathrm{hi}}$ & $2.2 \pm 1.6^{\mathrm{b}}$ & $0.94 \pm 0.1^{\mathrm{bcd}}$ & $0.60 \pm 0.4^{b c d}$ \\
\hline SW 08-190001-12 & - & - & $1.24 \pm 0.1^{\mathrm{ab}}$ & $1.18 \pm 0.2^{\mathrm{ab}}$ & $0.21 \pm 0.22^{c}$ & $1.0+0.1^{\text {cdef }}$ & $0.5 \pm 0.3^{\text {def }}$ & $0.82 \pm 0.0^{\mathrm{cd}}$ & $0.55 \pm 0.4^{b c d}$ \\
\hline SW 08-190001-1 & - & - & $1.16 \pm 0.1^{\mathrm{ab}}$ & $1.20 \pm 0.1^{\mathrm{ab}}$ & $0.25 \pm 0.16^{b c}$ & $0.8 \pm 0.3^{\text {efg }}$ & $1.1 \pm 0.7^{\text {cde }}$ & $0.97 \pm 0.2^{\mathrm{bcd}}$ & $0.33 \pm 0.6^{\mathrm{cd}}$ \\
\hline SW 08-190002-16 & - & - & $1.12 \pm 0.1^{\mathrm{ab}}$ & $1.04 \pm 0.0^{\mathrm{ab}}$ & $0.26 \pm 0.22^{b c}$ & $0.4 \pm 0.2^{\mathrm{fgh}}$ & $-1.3 \pm 0.5^{g}$ & $1.19 \pm 0.2^{\mathrm{abc}}$ & $0.58+0.6^{\mathrm{bcd}}$ \\
\hline SW 08-190002-5 & - & - & $1.22 \pm 0.1^{\mathrm{ab}}$ & $1.16 \pm 0.1^{\mathrm{ab}}$ & $0.28 \pm 0.24^{b c}$ & $1.1 \pm 0.5^{\text {bcdef }}$ & $0.2 \pm 0.0^{\text {ef }}$ & $1.03 \pm 0.4^{\mathrm{bcd}}$ & $0.63 \pm 0.6^{\mathrm{abcd}}$ \\
\hline SW 08-190001-6 & - & - & $1.09 \pm 0.1^{\mathrm{ab}}$ & $1.22 \pm 0.2^{\mathrm{ab}}$ & $0.29 \pm 0.26^{b c}$ & $1.4 \pm 0.1^{\mathrm{bcd}}$ & $3.6 \pm 0.4^{a}$ & $1.27 \pm 0.1^{\mathrm{ab}}$ & $0.72 \pm 0.4^{\mathrm{abcd}}$ \\
\hline SW 08-190002-11 & - & - & $1.09 \pm 0.1^{\mathrm{ab}}$ & $1.16 \pm 0.1^{\mathrm{ab}}$ & $0.29 \pm 0.19^{b c}$ & $0.7 \pm 0.4^{\text {efg }}$ & $-0.5 \pm 0.1^{g}$ & $1.19 \pm 0.2^{\mathrm{abc}}$ & $0.77 \pm 0.4^{\mathrm{abcd}}$ \\
\hline Oase ${ }^{(\mathrm{RC})}$ & - & - & $1.01 \pm 0.0^{b}$ & $1.03 \pm 0.0^{\mathrm{ab}}$ & $0.37 \pm 0.63^{c}$ & $0.0 \pm 0.2^{\mathrm{hi}}$ & $1.0 \pm 0.4^{\text {cde }}$ & $0.36 \pm 0.1^{\text {ef }}$ & $0.42 \pm 0.2^{\mathrm{bcd}}$ \\
\hline SW 08-190002-8 & - & - & $1.01 \pm 0.0^{b}$ & $1.01 \pm 0.0^{b}$ & $0.55 \pm 0.93^{c}$ & $0.7 \pm 0.5^{\mathrm{efg}}$ & $0.6 \pm 0.3^{\text {def }}$ & $1.06 \pm 0.1^{\mathrm{abcd}}$ & $0.54 \pm 0.4^{\mathrm{bcd}}$ \\
\hline SW 08-190001-11 & - & - & $1.26 \pm 0.1^{\mathrm{ab}}$ & $1.48+0.3^{\mathrm{ab}}$ & $1.09 \pm 1.08^{\mathrm{ab}}$ & $-0.4 \pm 0.0^{i}$ & $0.9 \pm 0.5^{\text {cde }}$ & $1.18 \pm 0.2^{\mathrm{abc}}$ & $0.99 \pm 0.1^{\mathrm{abc}}$ \\
\hline Falcon $^{(\mathrm{SC})}$ & - & - & $1.31 \pm 0.2^{\mathrm{ab}}$ & $1.36 \pm 0.1^{\mathrm{ab}}$ & $1.47 \pm 1.78^{\mathrm{ab}}$ & $1.3 \pm 1.1^{\text {bcde }}$ & $0.3 \pm 0.5^{\mathrm{ef}}$ & $1.49 \pm 0.1^{\mathrm{a}}$ & $1.32 \pm 0.2^{\mathrm{a}}$ \\
\hline Laser $^{(\mathrm{SC})}$ & - & - & $1.68 \pm 0.4^{\mathrm{a}}$ & $1.53 \pm 0.2^{\mathrm{a}}$ & $10.37 \pm 16.07^{\mathrm{a}}$ & $2.4 \pm 0.1^{\mathrm{a}}$ & $2.4 \pm 0.9^{b}$ & $1.05 \pm 0.1^{\mathrm{abcd}}$ & $1.06 \pm 0.1^{\mathrm{ab}}$ \\
\hline
\end{tabular}

DI, shoot disease index. RDI, root disease index. NNA, net normalized AUDPC. NRS, normalized relative stunting. VLDNA, Verticillium longisporum DNA (ng/g) in hypocotyl at BBCH80. ND, no data due to loss of plants by hard winter. (RC), resistant control. (SC), susceptible control. ${ }^{*}$, not a double haploid line. For all parameters mean \pm standard deviation data is shown. Greenhouse results are mean of 60 plants derived from three independent experiments. In outdoor experiment, vernalized seedlings inoculated with VL were transplanted to pots and grown outdoor (open air condition). Genotypes are sorted according to field VLDNA quantification results that showed better correlation with other field, outdoor and GH parameters and across locations. For each parameter, means followed by the same letter are not significantly different at $p=0.05$. Mean values under the parameters indicated by the superscript 'ns' are not significantly different at $p=0.05$. 
Table 2.6 Correlation between field performance and greenhouse or outdoor screening results of 14 Brassica napus double haploid lines and 4 reference varieties evaluated for Verticillium longisporum resistance in three successive years (2011, 2012 and 2013).

A. Correlation between field performance in Fehmarn with outdoor and greenhouse results

\begin{tabular}{|c|c|c|c|c|c|c|c|c|}
\hline \multirow[b]{2}{*}{ Trial } & \multirow[b]{2}{*}{ Parameters } & \multicolumn{7}{|c|}{ Field trial (Fehmarn) } \\
\hline & & SDI 2011 & RDI 2011 & SDI 2012 & RDI 2012 & VLDNA 2012 & SDI 2013 & RDI 2013 \\
\hline \multirow{6}{*}{ 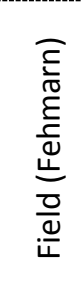 } & RDI 2011 & $0.66^{* *}$ & & & & & & \\
\hline & SDI 2012 & $0.50^{*}$ & $0.28^{\mathrm{ns}}$ & & & & & \\
\hline & RDI 2012 & $0.34^{\mathrm{ns}}$ & $0.12^{\mathrm{ns}}$ & $0.85^{* * *}$ & & & & \\
\hline & VLDNA 2012 & $0.53 *$ & $0.31^{\mathrm{ns}}$ & $0.16^{\mathrm{ns}}$ & $0.10^{\mathrm{ns}}$ & & & \\
\hline & SDI 2013 & $0.26^{\mathrm{ns}}$ & $0.32^{\mathrm{ns}}$ & $0.09^{\text {ns }}$ & $-0.18^{\mathrm{ns}}$ & $0.14^{\mathrm{ns}}$ & & \\
\hline & RDI 2013 & $0.32^{\mathrm{ns}}$ & $0.17^{\mathrm{ns}}$ & $0.41^{\mathrm{ns}}$ & $0.19^{\mathrm{ns}}$ & $0.15^{\mathrm{ns}}$ & $0.84^{* * *}$ & \\
\hline \multirow{2}{*}{ ๑ } & NNA & $0.42^{\mathrm{ns}}$ & $0.51 *$ & $-0.13^{\mathrm{ns}}$ & $-0.21^{\mathrm{ns}}$ & $0.51^{*}$ & $0.38^{\mathrm{ns}}$ & $0.27^{\mathrm{ns}}$ \\
\hline & NRS & $0.04^{\mathrm{ns}}$ & $-0.03^{\text {ns }}$ & $-0.17^{\text {ns }}$ & $0.00^{\mathrm{ns}}$ & $0.19^{\mathrm{ns}}$ & $-0.31^{\text {ns }}$ & $-0.38^{\text {ns }}$ \\
\hline \multirow{2}{*}{ 㞼 } & NNA & $0.27^{\mathrm{ns}}$ & $-0.16^{\mathrm{ns}}$ & $0.02^{\mathrm{ns}}$ & $-0.12^{\mathrm{ns}}$ & $0.33^{\mathrm{ns}}$ & $0.54 *$ & $0.55^{*}$ \\
\hline & NRS & $0.48^{*}$ & $0.23^{\mathrm{ns}}$ & $0.15^{\mathrm{ns}}$ & $0.14^{\mathrm{ns}}$ & $0.62 * *$ & $0.32^{\mathrm{ns}}$ & $0.29^{\text {ns }}$ \\
\hline
\end{tabular}

B. Correlation between field performance in Svalöv with outdoor and greenhouse results

\begin{tabular}{|c|c|c|c|c|c|c|c|}
\hline \multirow[b]{2}{*}{ Trial } & \multirow[b]{2}{*}{ Parameters } & \multicolumn{6}{|c|}{ Field trial (Svalöv) } \\
\hline & & SDI 2011 & RDI 2011 & SDI 2012 & RDI 2012 & VLDNA 2012 & 2013 (ND) \\
\hline \multirow{4}{*}{$\begin{array}{l}\frac{3}{3} \\
\frac{0}{\pi} \\
\frac{\sqrt{n}}{0} \\
\frac{0}{U} \\
\frac{0}{4}\end{array}$} & RDI 2011 & $0.73^{* *}$ & & & & & \\
\hline & SDI 2012 & $0.26^{\mathrm{ns}}$ & $0.09^{\text {ns }}$ & & & & \\
\hline & RDI 2012 & $0.03^{\mathrm{ns}}$ & $-0.16^{n} s$ & $0.91 * * *$ & & & \\
\hline & VLDNA 2012 & $0.11^{\mathrm{ns}}$ & $-0.09^{n s}$ & $0.17^{\mathrm{ns}}$ & $0.23^{\mathrm{ns}}$ & & \\
\hline \multirow{2}{*}{ จิ } & NNA & $-0.08^{\mathrm{ns}}$ & $-0.17^{\mathrm{ns}}$ & $-0.02^{\mathrm{ns}}$ & $0.05^{\mathrm{ns}}$ & $0.43^{\mathrm{ns}}$ & - \\
\hline & NRS & $0.18^{\mathrm{ns}}$ & $-0.05^{n s}$ & $0.191^{\mathrm{ns}}$ & $0.11^{\mathrm{ns}}$ & $0.08^{\text {ns }}$ & - \\
\hline \multirow{2}{*}{ I } & NNA & $0.30^{\mathrm{ns}}$ & $0.13^{\text {ns }}$ & $0.36 n^{s}$ & $0.37^{\mathrm{ns}}$ & $0.25^{\mathrm{ns}}$ & - \\
\hline & NRS & $0.12^{\mathrm{ns}}$ & $-0.11^{\mathrm{ns}}$ & $0.30^{\mathrm{ns}}$ & $0.41^{\mathrm{ns}}$ & $0.56 *$ & - \\
\hline
\end{tabular}

C. Correlation between field performance in Göttingen with outdoor and greenhouse results

\begin{tabular}{|c|c|c|c|c|c|c|}
\hline \multirow[b]{2}{*}{ Trial } & \multirow[b]{2}{*}{ Parameters } & \multicolumn{5}{|c|}{ Field trial (Göttingen) } \\
\hline & & 2011 (ND) & 2012 (ND) & SDI 2013 & RDI 2013 & VLDNA 2013 \\
\hline \multirow{2}{*}{$\frac{\overline{0}}{\frac{0}{4}}$} & RDI 2013 & - & - & $0.83 * * *$ & & \\
\hline & VLDNA 2013 & - & - & $0.85^{* * *}$ & $0.68 * *$ & \\
\hline \multirow{2}{*}{ ○ } & NNA & - & - & $0.46^{\mathrm{ns}}$ & $0.21^{\mathrm{ns}}$ & $0.55^{*}$ \\
\hline & NRS & - & - & $0.17^{\mathrm{ns}}$ & $0.34^{\mathrm{ns}}$ & $0.33^{\text {ns }}$ \\
\hline \multirow{2}{*}{ 壱 } & NNA & - & - & $0.09^{\mathrm{ns}}$ & $0.28^{\mathrm{ns}}$ & $0.13^{\mathrm{ns}}$ \\
\hline & NRS & - & - & $0.44^{\mathrm{ns}}$ & $0.56^{*}$ & $0.40^{\mathrm{ns}}$ \\
\hline
\end{tabular}

Pearson correlation analysis was performed using product-moment procedure of STATISTICA (Version 10 StatSoft, inc. 2011). Minus signs indicate negative correlations. Significant correlations are indicated in bold font. *, significant at $\mathrm{P}=0.05 .{ }^{* *}$, significant at $\mathrm{P}=0.01$. ${ }^{* * *}$, significant at $\mathrm{P}=0.001$. ns, not significant. $\mathrm{OD}$, outdoor experiment. GH, greenhouse experiment. SDI, Shoot disease index. RDI, Root disease index. NNA, Net normalized AUDPC. NRS, Normalized net relative stunting. VLDNA, Verticillium longisporum DNA in hypocotyl tissue. ND, no data due to loss of plants by hard winter. 
Table 2.7 Pearson correlations for field performance of Verticillium longisporum infected Brassica napus double haploid lines in three locations.

\begin{tabular}{|c|c|c|c|}
\hline \multirow[t]{2}{*}{ Parameters } & \multicolumn{3}{|c|}{ Field trial 2010/11 } \\
\hline & Svalöv SDI & Svalöv RDI & Göttingen (ND) \\
\hline Fehmarn SDI & $-0.20^{\mathrm{ns}}$ & $-0.04^{\mathrm{ns}}$ & - \\
\hline Fehmarn RDI & $-0.44^{\mathrm{ns}}$ & $-0.41^{\mathrm{ns}}$ & - \\
\hline \multirow[t]{2}{*}{ Parameters } & \multicolumn{3}{|c|}{ Field trial 2011/12 } \\
\hline & Svalöv SDI & Svalöv RDI & Svalöv VLDNA \\
\hline Fehmarn SDI & $0.37^{\mathrm{ns}}$ & $0.45^{\mathrm{ns}}$ & $-0.10^{\mathrm{ns}}$ \\
\hline Fehmarn RDI & $0.31^{\mathrm{ns}}$ & $0.37^{\mathrm{ns}}$ & $-0.12^{\mathrm{ns}}$ \\
\hline Fehmarn VLDNA & $0.32^{\mathrm{ns}}$ & $0.30^{\mathrm{ns}}$ & $0.86^{* * *}$ \\
\hline \multirow[t]{2}{*}{ Parameters } & \multicolumn{3}{|c|}{ Field trial 2012/13 } \\
\hline & Göttingen SDI & Göttingen RDI & Göttingen VLDNA \\
\hline Fehmarn SDI & $0.10^{\mathrm{ns}}$ & $0.00^{\text {ns }}$ & $0.03^{\mathrm{ns}}$ \\
\hline Fehmarn RDI & $0.07^{\mathrm{ns}}$ & $-0.02^{\mathrm{ns}}$ & $-0.06^{\mathrm{ns}}$ \\
\hline Svalöv (ND) & - & - & - \\
\hline
\end{tabular}

Pearson correlation analysis was performed using product-moment procedure of STATISTICA (Version 10 StatSoft, inc. 2011).Phenotypic and molecular disease screening results obtained from 14 Brassica napus double haploid lines and four reference varieties used in three consecutive field screening trials were used. Minus signs indicate negative correlations. Significant correlations are indicated in bold font. ${ }^{*}$, significant at $\mathrm{P}=0.05$. ${ }^{* *}$, significant at $\mathrm{P}=0.01 . * * *$, significant at $\mathrm{P}=0.001$. ns, not significant. $\mathrm{OD}$, outdoor experiment. $\mathrm{GH}$, greenhouse experiment. SDI, shoot disease index. RDI, root disease index. VLDNA, Verticillium longisporum DNA in hypocotyl tissue. ND, no data due to loss of plants by hard winter.

\subsubsection{Different sets of B. napus lines screened for $V$. longisporum resistance under field conditions for only one season (year)}

Here, the field screening results of $B$. napus lines evaluated at different locations are presented in a year-by-year manner. During each season (2010/11, 2011/12 and 2012/13), different sets of 22 B. napus genotypes were tested.

For the DH lines evaluated in $\mathbf{2 0 1 0 / 1 1}$ field trials in two locations (Fehmarn and Svalöv), disease assessment was done by SDI and RDI scoring. Of these measurements, the only parameter that showed significant differences among genotypes was Fehmarn SDI (Table 2.8) and this parameter was the only one that significantly correlated $(r=0.51)$ with greenhouse NRS (Table 2.11A). The remaining measurements (Fehmarn RDI and Svalöv SDI and RDI) were not significantly different among all lines and reference varieties (Table 2.8). Apart from this, significant correlations were found between SDI and RDI in each location independently (Table 2.11A). Considering the only two parameters that showed significant correlations, DH lines DH196, DH69, and DH138, DH165 performed more or less similar in resistance to the reference variety Oase. In contrast, lines $\mathrm{DH} 282$, DH123, DH290, DH152 and DH16 showed a poor performance comparable to the susceptible reference variety Laser. 
A similar trend was observed for the set of DH lines screened during 2011/12 in Fehmarn and Svalöv. The analysis of SDI and RDI data obtained from both locations showed no significant differences. In contrast, data obtained from qPCR analysis of samples collected from both locations showed significant differentiation (Table 2.9). In Fehmarn, this data significantly differed among the reference varieties. Furthermore, except for DSV-DH-Ver-9(1605-2) and DSV-DH-Ver-19(1605-2), all other DH lines had significantly lower levels of VL DNA compared to both susceptible controls. Regarding the results in Svalöv, despite the presence of a double as high level of VL DNA in the susceptible controls, the difference between resistant and susceptible controls was not statistically significant. Nevertheless, $13 \mathrm{DH}$ lines with significantly lower amounts of VL DNA than in both susceptible controls were found. Of these, five of them [DSV-DH-Ver-7(1575-2), DSV-DH-Ver14(1605-2), DSV-DH-Ver-5(1575-1), DSV-DH-Ver-22(1605-2) and DSV-DH-Ver-21(1605-1)] had significantly less VL DNA than both resistant controls. Correlation analysis of the results from DH lines screened in 2011/12 indicated that qPCR data was the highest correlated parameter across locations and between greenhouse results (Table 2.11B). Thus, qPCR results in Svalöv were significantly and positively correlated with Fehmarn VLDNA $(r=0.70)$, greenhouse NNA $(r=0.59)$ and greenhouse NRS $(r=0.48)$ results. Similarly, significant correlations of Fehmarn VL DNA with Svalöv VL DNA $(r=0.70)$, greenhouse NNA $(r=0.40)$ and greenhouse RNS $(r=0.50)$ results were found. Moreover, there was a significant correlation between shoot and root DI assessments in Fehmarn (0.73) and in Svalöv ( $r=0.99$ ). Similar to most previously described field results, Svalöv disease index measurements showed no significant correlation with any of the other field or greenhouse parameters. Surprisingly, Fehmarn SDI and RDI measurements had significant negative correlations with Fehmarn VL DNA, Svalöv VL DNA and greenhouse NNA and NRS results (Table 2.11B). These correlations completely contradict the above mentioned qPCR (VL DNA) data of the same experiment (obtained from the same set of genotypes) that showed significant positive correlations with Svalöv VL DNA and greenhouse NNA and NRS results. Greenness of plant tissue at the time of harvesting stubble samples might potentially affect the accuracy of disease index scoring. Green stubble samples on which microsclerotia had not yet formed might possibly be scored as low disease index and this might possibly lead to the aforementioned negative correlation of Fehmarn disease index measurements with other field and greenhouse results. Nevertheless, if further work needs to be done on the lines tested in 2011/12 in Fehmarn and Svalöv, priority should be given to the following 12 lines [DSV-DH-Ver-21(1605-1), DSV-DH-Ver-22(1605-2), DSV-DH-Ver-20(1605-3), DSVDH-Ver-16(1605-2), DSV-DH-Ver-8(1605-2), DSV-DH-Ver-10(1605-2), DSV-DH-Ver-11(1605-2), DSVDH-Ver-14(1605-2), DSV-DH-Ver-12(1605-2), DSV-DH-Ver-4(1575-3), DSV-DH-Ver-6(1575-1) and DSV-DH-Ver-18(1605-2)] that have shown better performance than the resistant reference varieties both in the field and under greenhouse conditions (Table 2.9). 
Results in 2012/13 with 22 B. napus lines were only obtained from Göttingen and Fehmarn since the plants in Svalöv were lost due to the strong winter (Table 2.10). Regarding the results from Fehmarn, only data from disease index assessments (SDI and RDI) were available and both parameters showed no significant differences among the genotypes. In Göttingen, disease assessment was carried out by disease index scoring and quantification of VL DNA. With both parameters, genotypes displayed significant differences. Regarding SDI, except for the susceptible control cultivar Laser and the DH line DM12-02 that showed a significantly higher SDI than KWS195, the differences among the rest of the reference controls and $B$. napus lines was not significant. Similarly, the only observed significant difference with regard to RDI was between the susceptible cultivar Laser and KWS195.

Interestingly, a better differentiation of the lines was achieved by quantification of VL DNA. In particular, the resistant and susceptible reference varieties showed significant differences. Accordingly, the highest VL DNA was quantified from Laser $(1.53 \mathrm{ng} / \mathrm{g})$ and this amount was significantly higher than in Oase and Express. VL DNA in cultivar Falcon was also significantly higher than in Express, from which the least amount of VL DNA $(0.08 \mathrm{ng} / \mathrm{g})$ was quantified. Among the remaining lines, SW12-4, NICK220, NICK221, KWS200, SW08-190002-17 and SYN10091232 had significantly lower VL DNA than both susceptible controls. In general, the observed disease levels in Göttingen were very low resulting in a disease index less than 2.0 (very slight infestation) and VL DNA below $0.5 \mathrm{ng} / \mathrm{g}$ for most genotypes. Nevertheless, unlike all previously described field results and despite a very low level of infection, data obtained from the 22 B. napus lines and 4 reference varieties screened in 2012/13 in Göttingen showed significant correlations between field disease index (SDI and RDI), field VL DNA and greenhouse (NNA and NRS) results (Table 2.11C). One of the possible explanations for the rare significant correlation between field disease index and greenhouse results may possibly be the uniform exposure of all genotypes to the same type and amount of inoculum and the use of artificial inoculation. Considering these correlations, promising lines to be suggested for further research are NICK 221, NICK 219, SYN10091232, SW08-190002-2, DSV, SW122 KWS 195, SW08-190002-25 and KWS 187. The ones that showed weak performance comparable to the susceptible controls were DM12-01 and DM12-01 (Table 2.10).

In summary, the field experimental results described in the previous two sections of this chapter (2.4.4.1 and 2.4.4.2) provide evidence that field evaluation of $V$. longisporum resistance in OSR based on assessment of stubble disease index seems to be inconsistent. With this parameter, a high variability among results of the same season trials conducted in different locations was found. Moreover, the same genotype frequently showed considerably different levels of disease in different season's trials of the same location. Furthermore, despite the variation in several agro-ecological 
Table 2.8 Response of 22 Brassica napus double haploid lines and four reference varieties evaluated for Verticillium longisporum resistance under field conditions in the $\mathbf{2 0 1 0 / 1 1}$ growing season. Comparisons of field performance across locations and field vs greenhouse screening results are shown.

\begin{tabular}{|c|c|c|c|c|c|c|c|}
\hline \multirow{2}{*}{ Genotype code } & \multicolumn{2}{|c|}{ Fehmarn } & \multirow{2}{*}{$\begin{array}{l}\text { Göttingen } \\
\text { (ND) }\end{array}$} & \multicolumn{2}{|c|}{ Svalöv } & \multicolumn{2}{|c|}{ Greenhouse } \\
\hline & SDI & $\mathrm{RDI}^{\mathrm{ns}}$ & & $S D I^{\text {ns }}$ & $\mathrm{RDI}^{\mathrm{ns}}$ & NNA & NRS \\
\hline $\mathrm{DH} 88$ & $2.57 \pm 0.0^{\mathrm{ef}}$ & $1.46+0.0$ & - & $2.70+0.6$ & $2.48+0.4$ & $1.52 \pm 0.7^{\mathrm{bc}}$ & $0.90+0.5^{b c}$ \\
\hline $\mathrm{DH} 69$ & $2.68+0.2^{\mathrm{def}}$ & $1.50+0.1$ & - & $2.57+0.1$ & $2.56+0.2$ & $0.92+0.4^{\mathrm{fgh}}$ & $0.64+0.3^{\mathrm{ab}}$ \\
\hline DH 179 & $2.80+0.3^{\text {cdef }}$ & $1.60+0.3$ & - & $2.45+0.4$ & $2.50+0.5$ & $1.19+0.8^{\text {cdefg }}$ & $0.96+0.5^{f g}$ \\
\hline $\mathrm{DH} 165$ & $2.68 \pm 0.5^{\text {def }}$ & $1.52 \pm 0.2$ & - & $2.82 \pm 0.6$ & $2.76 \pm 1.1$ & $0.80 \pm 0.3^{\text {fghij }}$ & $0.62+0.2^{\mathrm{ab}}$ \\
\hline $\mathrm{DH} 272$ & $2.50 \pm 0.7^{\text {ef }}$ & $1.63 \pm 0.2$ & - & $2.96 \pm 0.1$ & $3.00 \pm 0.1$ & $0.94 \pm 0.5^{\mathrm{fgh}}$ & $0.73+0.4^{\mathrm{fg}}$ \\
\hline Oase $e^{(R C)}$ & $2.80 \pm 0.4^{\text {bcdef }}$ & $1.68 \pm 0.3$ & - & $2.86 \pm 0.8$ & $2.92 \pm 0.6$ & $0.24 \pm 0.1^{k}$ & $0.27+0.2^{f}$ \\
\hline DH 138 & $2.68+0.1^{\text {def }}$ & $1.68+0.0$ & - & $2.04 \pm 1.4$ & $1.95+1.3$ & $0.70 \pm 0.3^{\text {hij }}$ & $0.48+0.2^{\mathrm{cd}}$ \\
\hline DH 152 & $3.25+0.2^{\mathrm{abcd}}$ & $1.68+0.1$ & - & $3.12+0.5$ & $2.74+0.4$ & $1.85+0.6^{\mathrm{ab}}$ & $1.43+0.4^{c d}$ \\
\hline DH 126 & $2.93+0.4^{\text {abcdef }}$ & $1.70+0.1$ & - & $2.18 \pm 1.1$ & $2.04 \pm 1.1$ & $1.42+0.5^{\mathrm{bcd}}$ & $1.22+0.4^{e f}$ \\
\hline Express $^{(\mathrm{RC})}$ & $2.85+0.4^{\text {cdef }}$ & $1.76 \pm 0.1$ & - & $1.28 \pm 0.2$ & $1.56 \pm 0.1$ & $0.56 \pm 0.5^{\mathrm{ijk}}$ & $0.68+0.4^{\text {de }}$ \\
\hline Laser ${ }^{(\mathrm{SC})}$ & $3.40 \pm 0.1^{\mathrm{abcde}}$ & $1.78 \pm 0.1$ & - & $2.48 \pm 1.0$ & $2.44 \pm 1.0$ & $0.98 \pm 0.5^{\text {efgh }}$ & $0.96+0.5^{c d}$ \\
\hline DH 123 & $3.23+0.2^{\mathrm{abcd}}$ & $1.78+0.1$ & - & $2.28+0.9$ & $2.25+0.8$ & $1.58+0.6^{b c}$ & $1.36+0.4^{\mathrm{ab}}$ \\
\hline DH 290 & $3.23 \pm 0.2^{\mathrm{abcd}}$ & $1.78 \pm 0.4$ & - & $2.99 \pm 0.2$ & $3.04 \pm 0.1$ & $1.84 \pm 0.4^{\mathrm{ab}}$ & $1.43+0.4^{\mathrm{ab}}$ \\
\hline DH 196 & $2.47 \pm 0.3^{f}$ & $1.80 \pm 0.2$ & - & $2.93+0.2$ & $2.68+0.2$ & $0.74 \pm 0.3^{\text {ghij }}$ & $0.45+0.2^{\text {de }}$ \\
\hline DH 294 & $3.06+0.1^{\text {abcde }}$ & $1.80 \pm 0.2$ & - & $2.12 \pm 0.4$ & $2.00 \pm 0.2$ & $0.49+0.3^{\mathrm{jk}}$ & $0.47+0.2^{\text {de }}$ \\
\hline DH 359 & $2.52 \pm 0.3^{\text {ef }}$ & $1.80 \pm 0.1$ & - & $1.91 \pm 0.7$ & $1.86+0.5$ & $1.05+0.5^{\text {defgh }}$ & $0.69+0.3^{\text {de }}$ \\
\hline Falcon ${ }^{(\mathrm{SC})}$ & $3.07 \pm 0.0^{\mathrm{ab}}$ & $1.82+0.1$ & - & $2.78+0.5$ & $2.28+0.2$ & $1.44 \pm 0.7^{\text {bcde }}$ & $1.32+0.6^{\mathrm{ab}}$ \\
\hline DH 101 & $2.88 \pm 0.3^{\text {abcdef }}$ & $1.82 \pm 0.1$ & - & $2.10 \pm 1.0$ & $2.09 \pm 0.8$ & $1.47 \pm 0.3^{b c}$ & $0.67+0.3^{f g}$ \\
\hline $\mathrm{DH} 108$ & $2.83+0.2^{\text {bcdef }}$ & $1.82 \pm 0.1$ & - & $3.24 \pm 0.2$ & $3.12 \pm 0.1$ & $1.02 \pm 0.3^{\text {defgh }}$ & $0.52+0.1^{\text {de }}$ \\
\hline DH 282 & $3.45+0.3^{a}$ & $1.82+0.1$ & - & $2.64+0.3$ & $2.40+0.2$ & $1.14+0.3^{\text {cdef }}$ & $0.98+0.3^{a}$ \\
\hline $\mathrm{DH} 16$ & $3.27+0.4^{\mathrm{abc}}$ & $1.86+0.0$ & - & $3.00+0.6$ & $3.00+0.4$ & $2.42+0.6^{\mathrm{a}}$ & $1.63+0.3^{\mathrm{fg}}$ \\
\hline DH 24 & $3.19 \pm 0.1^{\mathrm{abcd}}$ & $1.87 \pm 0.1$ & - & $2.60 \pm 0.3$ & $2.51 \pm 0.2$ & $2.20 \pm 0.7^{\mathrm{a}}$ & $1.47+0.5^{\mathrm{de}}$ \\
\hline $\mathrm{DH} 7$ & $3.21+0.0^{\mathrm{abcd}}$ & $1.88+0.1$ & - & $3.09+0.2$ & $2.88+0.1$ & $0.96+0.7^{\mathrm{fgh}}$ & $0.46+0.7^{\mathrm{de}}$ \\
\hline DH 140 & $3.29+0.4^{\mathrm{abc}}$ & $1.88+0.3$ & - & $2.12+1.4$ & $2.08+1.1$ & $0.84+0.4^{\mathrm{fghi}}$ & $0.73+0.4^{f g}$ \\
\hline DH 118 & $3.19 \pm 0.3^{\mathrm{abcd}}$ & $1.94+0.1$ & - & $3.36 \pm 0.3$ & $3.06+0.1$ & $0.88 \pm 0.4^{\mathrm{fgh}}$ & $0.73+0.3^{f g}$ \\
\hline DH 141 & $3.03+0.0^{\text {abcdef }}$ & $2.04+0.2$ & - & $3.22+0.1$ & $3.16+0.1$ & $0.81+0.2^{\text {fghi }}$ & $0.55+0.1^{\text {de }}$ \\
\hline
\end{tabular}

SDI, shoot disease index. RDI, root disease index. NNA, net normalized AUDPC. NRS, normalized relative stunting. ND, no data due to loss of plants by hard winter. (RC), resistant control. (SC), susceptible control. For all parameters mean \pm standard deviation data is shown. Greenhouse results are mean of 60 plants derived from three independent experiments. Genotypes are sorted according to Fehmarn SDI results that showed significant correlation with GH NRS results. For each parameter, means followed by the same letter are not significantly different at $p=0.05$. Mean values under parameters indicated by the superscript 'ns' are not significantly different at $p=0.05$. 
Table 2.9 Response of 22 Brassica napus double haploid lines and four reference varieties evaluated for Verticillium longisporum resistance under field conditions in the $\mathbf{2 0 1 1 / 1 2}$ growing season. Comparisons of field performance across locations and field vs greenhouse screening results are shown.

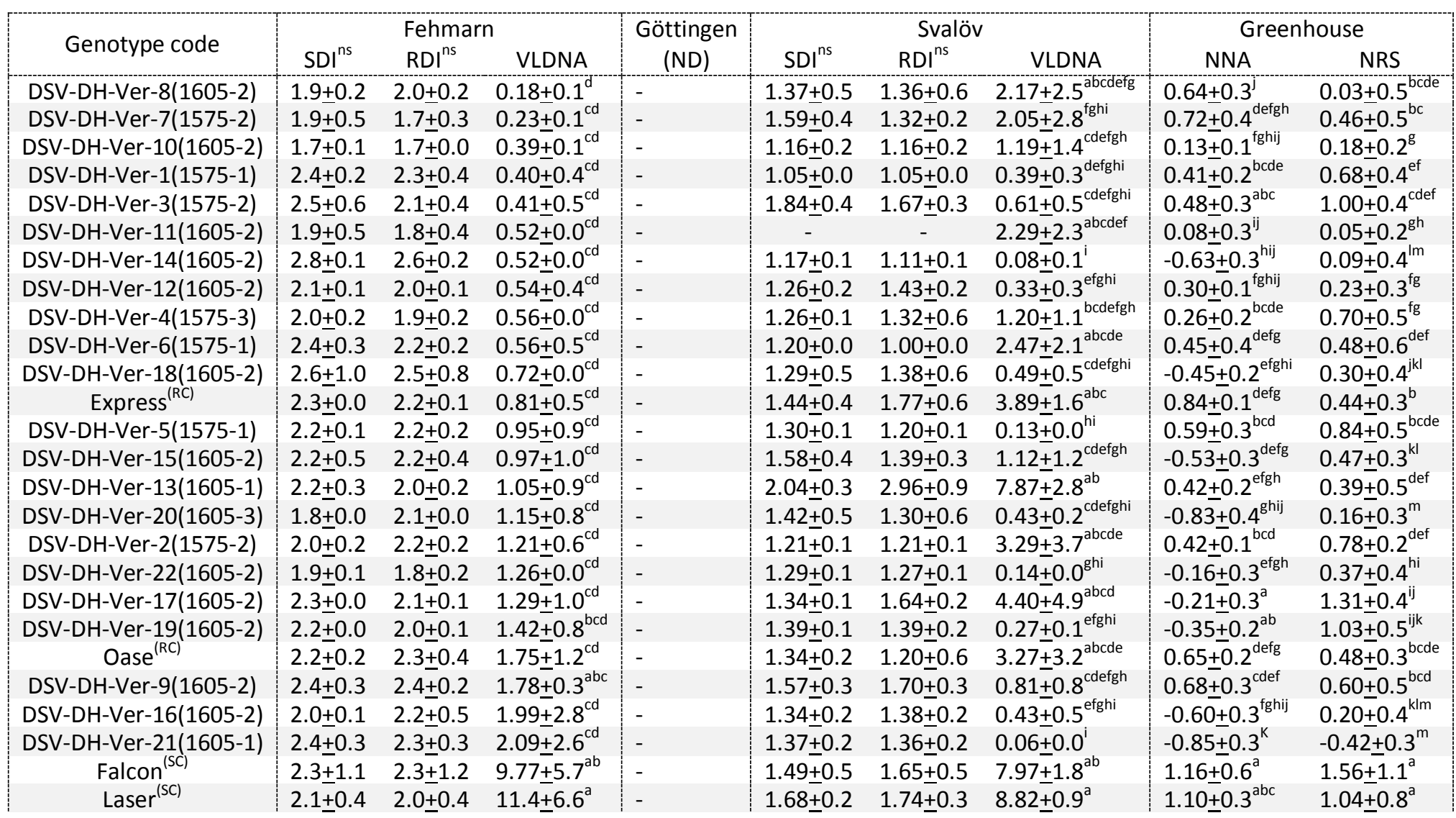

SDI, Shoot disease index. RDI, Root disease index. NNA, Net normalized AUDPC. NRS, Normalized relative stunting. VLDNA, Verticillium longisporum DNA (ng/g) in hypocotyl at BBCH80. ND, no data due to loss of plants by hard winter. (RC), resistant control. (SC), susceptible control. For all parameters mean \pm standard deviation data is shown. Greenhouse results are mean of 60 plants derived from three independent experiments. Genotypes are sorted according to Fehmarn VLDNA quantification results that showed significant correlation with Svalöv field, GH and OD screening results. For each parameter, means followed by the same letter are not significantly different at $p=0.05$. Mean values under parameters indicated by the superscript ' $n \mathbf{s}^{\prime}$ are not significantly different at $p=0.05$. 
Table 2.10 Response of 22 Brassica napus accessions and four reference varieties evaluated for Verticillium longisporum resistance under field conditions in the 2012/13 growing season. Comparisons of field performance across locations and field vs greenhouse screening results are shown.

\begin{tabular}{|c|c|c|c|c|c|c|c|c|}
\hline \multirow{2}{*}{ Genotype code } & \multicolumn{2}{|c|}{ Fehmarn } & \multicolumn{3}{|c|}{ Göttingen } & \multirow{2}{*}{$\begin{array}{c}\text { Svalöv } \\
\text { (ND) }\end{array}$} & \multicolumn{2}{|c|}{ Greenhouse } \\
\hline & $\mathrm{SDI} I^{\mathrm{ns}}$ & $\mathrm{RDI}^{\mathrm{ns}}$ & SDI & RDI & VLDNA & & NNA & NRS \\
\hline Express $^{(\mathrm{RC})}$ & $1.79+0.5$ & $1.90+0.1$ & $1.25+0.2^{\mathrm{ab}}$ & $1.17+0.1^{\mathrm{ab}}$ & $0.08+0.08^{e}$ & & $1.1+0.8^{\mathrm{bcd}}$ & $1.1+0.5^{\mathrm{abcd}}$ \\
\hline SW 12-4 & $2.24+0.4$ & $1.88+0.2$ & $1.24+0.2^{\mathrm{ab}}$ & $1.14+0.1^{\mathrm{ab}}$ & $0.15+0.12^{\mathrm{de}}$ & & $0.7+0.2^{\text {defg }}$ & $0.9+0.1^{\text {cdef }}$ \\
\hline NICK 220 & $2.65 \pm 0.4$ & $2.6 \pm 20.5$ & $1.21 \pm 0.1^{\mathrm{ab}}$ & $1.13 \pm 0.1^{\mathrm{ab}}$ & $0.17 \pm 0.11^{\text {de }}$ & & $0.7 \pm 0.2^{\text {defg }}$ & $0.4 \pm 0.1^{\mathrm{k}}$ \\
\hline NICK 221 & $1.90 \pm 0.3$ & $1.90 \pm 0.1$ & $1.20 \pm 0.2^{\mathrm{ab}}$ & $1.13 \pm 0.1^{\mathrm{ab}}$ & $0.17 \pm 0.13^{\mathrm{de}}$ & & $0.9 \pm 0.4^{\text {cde }}$ & $0.6 \pm 0.3^{\text {hijk }}$ \\
\hline KWS 200 & $2.31 \pm 0.3$ & $2.54 \pm 0.7$ & $1.16 \pm 0.1^{\mathrm{ab}}$ & $1.15 \pm 0.1^{\mathrm{ab}}$ & $0.18 \pm 0.14^{\mathrm{e}}$ & & $0.7 \pm 0.2^{\text {defg }}$ & $0.6 \pm 0.2^{\mathrm{hijk}}$ \\
\hline NICK 219 & $2.03 \pm 0.3$ & $1.74 \pm 0.4$ & $1.20 \pm 0.2^{\mathrm{ab}}$ & $1.17 \pm 0.2^{\mathrm{ab}}$ & $0.18+0.09^{\text {bcde }}$ & - & $0.8 \pm 0.3^{\text {cde }}$ & $0.9 \pm 0.2^{\text {defg }}$ \\
\hline SYN 10091232 & $2.16 \pm 0.8$ & $2.70 \pm 1.8$ & $1.06+0.1^{\mathrm{ab}}$ & $1.07 \pm 0.1^{\mathrm{ab}}$ & $0.20 \pm 0.23^{\mathrm{de}}$ & & $0.3+0.2^{i}$ & $0.5 \pm 0.2^{\mathrm{jk}}$ \\
\hline DM 12-02 & $2.33+0.1$ & $2.56+0.6$ & $1.51+0.2^{\mathrm{a}}$ & $1.46+0.2^{\mathrm{ab}}$ & $0.21+0.04^{\text {bcde }}$ & - & $1.4+0.4^{a}$ & $1.3+0.4^{\mathrm{ab}}$ \\
\hline DSV 1 & $2.27 \pm 0.2$ & $2.60 \pm 0.6$ & $1.40 \pm 0.2^{\mathrm{ab}}$ & $1.26 \pm 0.1^{\mathrm{ab}}$ & $0.21 \pm 0.21^{\text {bcde }}$ & 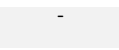 & $0.5 \pm 0.5^{\text {ghi }}$ & $0.6 \pm 0.4^{\mathrm{jk}}$ \\
\hline SW 08-190002-2 & $1.78 \pm 0.5$ & $1.82 \pm 0.3$ & $1.10 \pm 0.0^{\mathrm{ab}}$ & $1.19 \pm 0.1^{\mathrm{ab}}$ & $0.21 \pm 0.21^{\text {bcde }}$ & - & $0.8 \pm 0.3^{\text {cde }}$ & $0.5 \pm 0.3^{\text {efghi }}$ \\
\hline SYN 09033483 & $2.39 \pm 0.2$ & $2.34 \pm 0.9$ & $1.16 \pm 0.1^{\mathrm{ab}}$ & $1.18 \pm 0.1^{\mathrm{ab}}$ & $0.23 \pm 0.29^{\text {cde }}$ & - & $0.9 \pm 0.3^{\mathrm{bcd}}$ & $1.0 \pm 0.2^{\text {bcde }}$ \\
\hline DSV 3 & $2.41 \pm 0.9$ & $2.88 \pm 1.6$ & $1.20 \pm 0.1^{\mathrm{ab}}$ & $1.18 \pm 0.1^{\mathrm{ab}}$ & $0.29 \pm 0.23^{\text {bcde }}$ & - & $0.6 \pm 0.5^{\text {efgh }}$ & $0.7 \pm 0.5^{\text {ghij }}$ \\
\hline KWS 187 & $2.10 \pm 0.4$ & $2.44 \pm 0.8$ & $1.04 \pm 0.1^{\mathrm{ab}}$ & $1.12 \pm 0.2^{\mathrm{ab}}$ & $0.30+0.32^{\text {bcde }}$ & - & $0.4 \pm 0.3^{\mathrm{hi}}$ & $0.6+0.2^{i j k}$ \\
\hline SW 08-190002-17 & $2.06+0.1$ & $2.30+0.4$ & $1.13+0.1^{\mathrm{ab}}$ & $1.13+0.0^{\mathrm{ab}}$ & $0.31+0.34^{\mathrm{de}}$ & 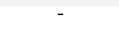 & $0.8+0.3^{\text {cde }}$ & $0.8+0.3^{j \mathrm{k}}$ \\
\hline SW 12-1 & $1.94+0.2$ & $2.08 \pm 0.1$ & $1.28+0.1^{\mathrm{ab}}$ & $1.22 \pm 0.2^{\mathrm{ab}}$ & $0.33+0.48^{\text {bcde }}$ & - & $0.6+0.3^{\text {efgh }}$ & $0.8 \pm 0.2^{\text {defgh }}$ \\
\hline SYN 11091465 & $2.23 \pm 0.4$ & $1.92 \pm 0.1$ & $1.29 \pm 0.1^{\mathrm{ab}}$ & $1.20 \pm 0.1^{\mathrm{ab}}$ & $0.33+0.22^{\text {bcde }}$ & - & $1.0 \pm 0.5^{\mathrm{bcd}}$ & $1.0 \pm 0.4^{\text {bcde }}$ \\
\hline DSV 2 & $2.23 \pm 0.6$ & $3.00 \pm 1.4$ & $1.10 \pm 0.1^{\mathrm{ab}}$ & $1.12 \pm 0.1^{\mathrm{ab}}$ & $0.34 \pm 0.36^{\text {bcde }}$ & - & $0.8 \pm 0.6^{\mathrm{def}}$ & $0.8 \pm 0.2^{\text {defgh }}$ \\
\hline SW $12-2$ & $2.26 \pm 0.4$ & $2.00 \pm 0.0$ & $1.08 \pm 0.1^{\mathrm{ab}}$ & $1.09 \pm 0.2^{\mathrm{ab}}$ & $0.34 \pm 0.26^{\text {bcde }}$ & - & $0.5 \pm 0.3^{\mathrm{fghi}}$ & $1.0 \pm 0.3^{\text {bcde }}$ \\
\hline Oase ${ }^{(R C)}$ & $1.34 \pm 0.4$ & $1.40 \pm 0.6$ & $1.01 \pm 0.0^{\mathrm{ab}}$ & $1.03 \pm 0.0^{\mathrm{ab}}$ & $0.37 \pm 0.63^{\text {cde }}$ & & $0.4 \pm 0.1^{\mathrm{hi}}$ & $0 . \overline{ \pm} \pm 0.2^{\text {fghij }}$ \\
\hline SW 12-3 & $1.89+0.3$ & $1.88+0.2$ & $1.13+0.1^{\mathrm{ab}}$ & $1.11+0.1^{\mathrm{ab}}$ & $0.38+0.44^{\text {bcde }}$ & - & $0.7+0.3^{\text {cdef }}$ & $0.9 \pm 0.2^{\text {cdef }}$ \\
\hline SW 08-190001-10 & $1.59 \pm 0.2$ & $1.94 \pm 0.1$ & $1.18 \pm 0.1^{\mathrm{ab}}$ & $1.21 \pm 0.1^{\mathrm{ab}}$ & $0.41 \pm 0.42^{\text {bcde }}$ & - & $0.9 \pm 0.2^{\mathrm{bcd}}$ & $0.7 \pm 0.1^{\text {ghij }}$ \\
\hline KWS 195 & $2.07 \pm 0.4$ & $2.38+0.9$ & $1.00 \pm 0.0^{\mathrm{b}}$ & $1.01+0.0^{\mathrm{b}}$ & $1.31+0.87^{\mathrm{ab}}$ & & $0.5+0.3^{\text {etghi }}$ & $0.8+0.2^{\text {etghi }}$ \\
\hline Falcon ${ }^{(S C)}$ & $2.18 \pm 0.5$ & $2.68 \pm 0.5$ & $1.31 \pm 0.2^{\mathrm{ab}}$ & $1.36 \pm 0.1^{\mathrm{ab}}$ & $1.47 \pm 1.78^{\mathrm{abc}}$ & . & $1.5+0.5^{\mathrm{a}}$ & $1.3+0.3^{\mathrm{a}}$ \\
\hline SW 08-190002-25 & $2.04 \pm 0.1$ & $2.16 \pm 0.2$ & $1.11 \pm 0.1^{\mathrm{ab}}$ & $1.11 \pm 0.1^{\mathrm{ab}}$ & $1.50 \pm 1.50^{\mathrm{abcd}}$ & - & $0.4 \pm 0.4^{\mathrm{hi}}$ & $0.8 \pm 0.3^{\text {efghi }}$ \\
\hline DM 12-01 & $2.21 \pm 0.2$ & $2.48 \pm 0.7$ & $1.62 \pm 0.4^{\mathrm{ab}}$ & $1.46 \pm 0.3^{\mathrm{ab}}$ & $1.76 \pm 3.15^{\mathrm{abcd}}$ & - & $1.3 \pm 0.4^{\mathrm{ab}}$ & $1.2 \pm 0.3^{\mathrm{abc}}$ \\
\hline Laser $^{(\mathrm{SC})}$ & $1.98 \pm 0.1$ & $1.96 \pm 0.1$ & $1.68 \pm 0.4^{a}$ & $1.53 \pm 0.2^{\mathrm{a}}$ & $10.3 \overline{7} \pm 16.07^{\mathrm{a}}$ & & $1.1+0.5^{\mathrm{abc}}$ & $1.0 \pm 0.4^{\text {bcde }}$ \\
\hline
\end{tabular}

SDI, shoot disease index. RDI, root disease index. NNA, net normalized AUDPC. NRS, normalized relative stunting. VLDNA, Verticillium longisporum DNA (ng/g) in hypocotyl at BBCH80. ND, no data due to loss of plants by hard winter. (RC), resistant control. (SC), susceptible control. For all parameters mean \pm standard deviation data is shown. Greenhouse results are mean of 60 plants derived from three independent experiments. Genotypes are sorted according to Göttingen VLDNA quantification results that showed significant correlation with field screening results. For each parameter, means followed by the same letter are not significantly different at $\mathrm{p}=0.05$. Mean values under parameters indicated by the superscript ' $n s^{\prime}$ are not significantly different at $p=0.05$. 
Table 2.11 Correlation analyses between field and greenhouse screening results of Brassica napus lines evaluated under field conditions for individual seasons (years).

A. Correlation between field and greenhouse results of 22 Brassica napus double haploid lines and four reference varieties screened during 2010/11 field trial.

\begin{tabular}{lccccc} 
& $\begin{array}{c}\text { Fehmarn } \\
\text { SDI }\end{array}$ & $\begin{array}{c}\text { Fehmarn } \\
\text { RDI }\end{array}$ & $\begin{array}{c}\text { Svalöv } \\
\text { SDI }\end{array}$ & $\begin{array}{c}\text { Svalöv } \\
\text { RDI }\end{array}$ & $\begin{array}{c}\text { GH } \\
\text { NNA }\end{array}$ \\
\hline Fehmarn RDI & $\mathbf{0 . 5 5 ^ { * * }}$ & & & & \\
Svalöv SDI & $0.15^{\text {ns }}$ & $0.15^{\text {ns }}$ & & & \\
Svalöv RDI & $0.10^{\text {ns }}$ & $0.12^{\text {ns }}$ & $0.95^{* * *}$ & & \\
Göttingen (ND) & - & - & - & - & - \\
GH NNA & $0.38^{\text {ns }}$ & $0.07^{\text {ns }}$ & $0.20^{\text {ns }}$ & $0.15^{\text {ns }}$ & \\
GH NRS & $\mathbf{0 . 5 1 ^ { * * }}$ & $0.06^{\text {ns }}$ & $0.09^{\text {ns }}$ & $0.03^{\text {ns }}$ & $\mathbf{0 . 9 0 * * *}$ \\
\hline
\end{tabular}

B. Correlation between field and greenhouse results of 22 Brassica napus double haploid lines and four reference varieties screened during 2011/12 field trial.

\begin{tabular}{|c|c|c|c|c|c|c|c|}
\hline & $\begin{array}{c}\text { Fehmarn } \\
\text { SDI }\end{array}$ & $\begin{array}{c}\text { Fehmarn } \\
\text { RDI }\end{array}$ & $\begin{array}{l}\text { Fehmarn } \\
\text { VLDNA }\end{array}$ & $\begin{array}{l}\text { Svalöv } \\
\text { SDI }\end{array}$ & $\begin{array}{c}\text { Svalöv } \\
\text { RDI }\end{array}$ & $\begin{array}{l}\text { Svalöv } \\
\text { VLDNA }\end{array}$ & $\begin{array}{c}\text { GH } \\
\text { NNA }\end{array}$ \\
\hline Fehmarn RDI & $0.73 * * *$ & & & & & & \\
\hline Fehmarn VLDNA & $-0.35^{\mathrm{ns}}$ & $-0.31^{\mathrm{ns}}$ & & & & & \\
\hline Svalöv SDI & $-0.01^{\mathrm{ns}}$ & $-0.06^{\mathrm{ns}}$ & $-0.09^{n} s$ & & & & \\
\hline Svalöv RDI & $-0.01^{\mathrm{ns}}$ & $0.06^{\mathrm{ns}}$ & $-0.09^{\mathrm{ns}}$ & $0.99 * * *$ & & & \\
\hline Svalöv VLDNA & $-0.52 * *$ & $-0.50 * *$ & $0.70 * * *$ & $0.02^{\mathrm{ns}}$ & $0.02^{\mathrm{ns}}$ & & \\
\hline Göttingen (ND) & - & - & - & - & - & - & - \\
\hline GH NNA & $-0.68 * * *$ & $-0.88 * * *$ & $0.40 *$ & $-0.03^{\mathrm{ns}}$ & $-0.05^{\mathrm{ns}}$ & $0.59 * *$ & \\
\hline GH NRS & $-0.48 *$ & $-0.48 *$ & $0.50 *$ & $-0.22^{\mathrm{ns}}$ & $-0.22^{\mathrm{ns}}$ & $0.48^{*}$ & $0.50 *$ \\
\hline
\end{tabular}

C. Correlation between field and greenhouse results of 22 Brassica napus accessions and four reference varieties screened during 2012/13 field trial.

\begin{tabular}{|c|c|c|c|c|c|c|}
\hline & $\begin{array}{c}\text { Fehmarn } \\
\text { SDI }\end{array}$ & $\begin{array}{c}\text { Fehmarn } \\
\text { RDI }\end{array}$ & $\begin{array}{l}\text { Göttingen } \\
\text { SDI }\end{array}$ & $\begin{array}{c}\text { Göttingen } \\
\text { RDI }\end{array}$ & $\begin{array}{l}\text { Göttingen } \\
\text { VLDNA }\end{array}$ & $\begin{array}{l}\text { GH } \\
\text { NNA }\end{array}$ \\
\hline Fehmarn RDI & $0.71 * * *$ & & & & & \\
\hline Göttingen SDI & $0.22^{\mathrm{ns}}$ & $0.10^{\mathrm{ns}}$ & & & & \\
\hline Göttingen RDI & $0.16^{\mathrm{ns}}$ & $0.15^{\mathrm{ns}}$ & $0.94 * * *$ & & & \\
\hline Göttingen VLDNA & $-0.08^{\mathrm{ns}}$ & $-0.10^{\text {ns }}$ & $0.58 * *$ & $0.58 * *$ & & \\
\hline Svalöv (ND) & - & - & - & - & - & - \\
\hline GH NNA & $0.10^{\mathrm{ns}}$ & $0.07^{\mathrm{ns}}$ & $0.68 * * *$ & $0.77^{* * *}$ & $0.26^{\mathrm{ns}}$ & \\
\hline GH NRS & $0.07^{\mathrm{ns}}$ & $-0.04^{\mathrm{ns}}$ & $0.53 * *$ & $0.58 * *$ & $0.24^{\mathrm{ns}}$ & $0.73^{* * *}$ \\
\hline
\end{tabular}

Pearson correlation analysis was performed using product-moment procedure of STATISTICA (Version 10 StatSoft, inc. 2011). For each season analysis, data obtained from 22 lines and 4 reference varieties were used. Minus signs indicate negative correlations. Significant correlations are indicated in bold font. ${ }^{*}$, significant at $\mathrm{P}=0.05 .{ }^{* *}$, significant at $\mathrm{P}=0.01{ }^{* * *}$, significant at $\mathrm{P}=0.001$. ns, not significant. $\mathrm{GH}$, greenhouse experiment. $\mathrm{OD}$, outdoor experiment. SDI, shoot disease index. RDI, root disease index. VLDNA, Verticillium longisporum DNA (ng/g) in hypocotyl. NNA, net normalized AUDPC. NRS, normalized net relative stunting. ND, no data due to loss of plants by hard winter. 
conditions across locations or seasons, in most cases (experiments) disease index results were hardly or only occasionally correlated with field qPCR as well as with NNA and NRS results from the greenhouse. Besides this, again in most cases the disease index showed no significant differences among genotypes, even between the well-known resistant and susceptible varieties. Understanding disease threshold levels by stubble rating at or after crop maturity stage (after grain harvest) is not useful anymore, particularly with regard to taking immediate control action. Because, as plants have already attained the maximum stage of development, application of any possible control measure (such as fungicide spray) at this time point may not be economical. Nevertheless, this does not mean that the information obtained from stubble disease index assessment is completely irrelevant. It rather provides valuable information on the amount of pathogen resting propagules (microsclerotia) in a certain field. Estimating the amount of inoculum in the soil is one of the basic and most important inputs required for forecasting disease pressure and this information will help to plan future production schemes such as decision making in crop rotation programs. In addition, the method can still be considered as one alternative of disease assessment in resistance screening studies.

The other alternative for measurement of VL disease severity in field-grown OSR plants is quantification of fungal biomass by qPCR. According to the results of the present multi-site study conducted in multiple seasons, it was found that this method provides more reliable information at a relatively earlier time point (plant growth stage). Furthermore, the results obtained from this parameter were mostly correlated with the results in controlled environments. More importantly, with this method, it was possible to detect even very slight levels of infection (up to few picograms of fungal DNA per gram of plant tissue). In conclusion, quantification of $V$. longisporum biomass by qPCR seems to be the most consistent, fast and possibly cheaper alternative for field disease survey or evaluation of resistance in OSR. Nevertheless, identifying the right time point (crop growth stage) at which VL can specifically and precisely be detected or quantified from field grown plants is crucial. From this knowledge, further extrapolation of future disease epidemics (ahead of crop maturity) is possible and this helps timely decision making on selection and application of possible disease control measures. This topic is partly addressed in the following chapter. 


\subsubsection{Determination of the critical crop growth stage for molecular detection and differential quantification of Verticillium longisporum disease severity in field grown winter oilseed rape plants}

In order to perform timely application of appropriate control measures, early detection and accurate quantification of VL infection in OSR production is very important. With this rationale, the present experiment was conducted with the objective of identifying the critical crop developmental growth stage for detection of $V$. longisporum infection from stem tissues of field grown winter OSR plants using qPCR. Furthermore, verifying the feasibility of this method as an alternative for assessment of VL disease severity or VL resistance in OSR was the focus of this experiment. For this purpose, stem samples of resistant and susceptible OSR cultivars from multi-locational field trials were collected at three growth stages (BBCH65, $\mathrm{BBCH} 70$ and $\mathrm{BBCH} 80$ ). The growth stages represent full flowering, fruit development and beginning of ripening stages, respectively. The amount of VL biomass in the DNA samples extracted from stem samples were quantified based on a standard curve (Fig. 2.5A) produced from known concentrations of VL genomic DNA. PCR amplification was performed using gene specific primer pairs targeting a 261bP ribosomal ITS (internal transcribed spacer) region in the $V$. longisporum genome (Knüfer, 2013). Furthermore, since the analysis was done on DNA samples extracted from field grown plants samples potentially harboring multiple pathogen species, specificity of PCR reaction was verified by melting curve analysis.

PCR results show high amplification efficiency (Fig. 2.5A) and a single melting peak (Fig. 2.5B) indicating the amplification of a single and specific DNA sequence. qPCR results revealed that irrespective of experimental locations, VL infection can be detected at growth stage BBCH65 (50\% flowering). However, fungal biomass in stem tissue at this growth stage was extremely low and consequently, no significant difference was found between susceptible and resistant varieties. At $\mathrm{BBCH70}$, relatively higher (than $\mathrm{BBCH} 65$ ) fungal biomass was quantified from both susceptible and resistant materials. Nevertheless, irrespective of experimental locations, no significant difference between resistant and susceptible materials was observed until this time point which marks the stage of pod development. At BBCH80, unlike earlier time points, relatively higher fungal biomass which significantly differed between resistant and susceptible materials was found (Fig. 2.6).

At this time point, VL DNA in the resistant cultivars Oase and Express in Göttingen (Fig. 2.6A) was 0.083 and $0.370 \mathrm{ng} / \mathrm{g}$, respectively. In this location, the highest amount of VL DNA $(10.4 \mathrm{ng} / \mathrm{g})$ which is significantly higher than the amounts detected in both resistant varieties was quantified from the 
susceptible Laser. In cultivar Falcon, 1.47ng/g which was significantly more than in Oase was found (Fig. 2.6A).
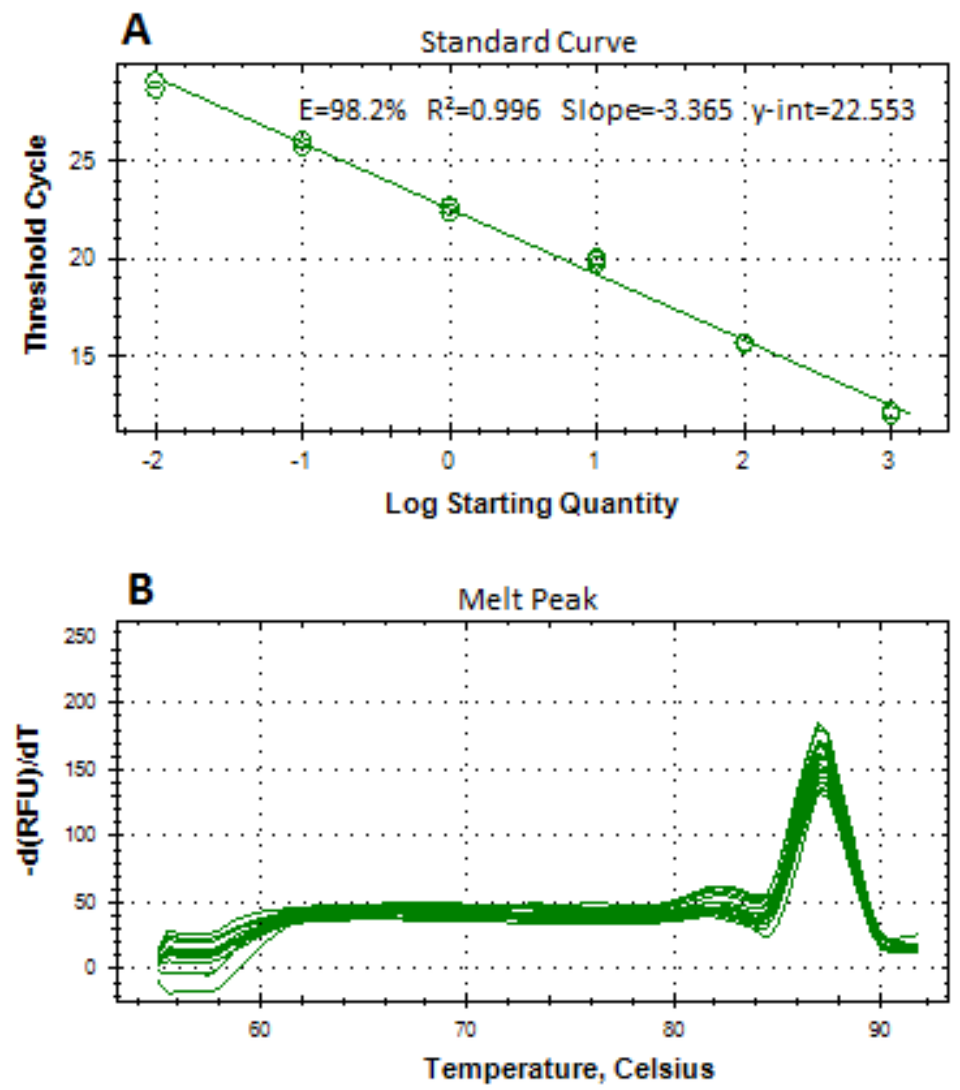

Figure 2.5 Standard curve (A) and melting peak (B) from real-time PCR amplification of tenfold dilution series of Verticillium longisporum genomic DNA. PCR was performed using ABsolute Blue SYBR Green Fluorescein Mix in a CFX384 real-time PCR system (Bio-Rad laboratories, Inc).

In Fehmarn (Fig. 2.6B), the highest level of infection (79.9ng) at BBCH80 which was significantly higher than all other varieties was found in Falcon. Regarding the other susceptible cultivar Laser, even though relatively high VL biomass $(22.0 \mathrm{ng} / \mathrm{g}$ ) was quantified, this amount was not significantly different from the quantity detected in the resistant material Oase (5.9ng/g) or Express (13.3ng/g).

In Svalöv (Fig. 2.6C) compared to the resistant cultivars, more than 300 fold VL DNA was quantified from the susceptible varieties. Accordingly, the highest infestation was found in Laser $(3252.6 \mathrm{ng} / \mathrm{g})$ and this amount was significantly higher than the amount quantified from Oase $(21.3 \mathrm{ng} / \mathrm{g})$ and Express (27.0ng/g). In cultivar Falcon, even though statistically insignificant, a still quite higher value (554.3ng/g) than in both resistant varieties was found.

The other important observation in this particular experiment was the variation of VL disease severity across locations. In general, the relatively highest infestation occurred in Svalöv followed by Fehmarn. In Göttingen, where the experiment was conducted with addition of inoculum (section 2.2.2.4), infestation levels were extremely low (Fig. 2.6). For instance, infection levels as measured by qPCR at BBCH80 were 7 (in Oase), 36 (in Express), 221 (in Laser), and 54 (in Falcon) fold higher VL DNA in Fehmarn compared 
to Göttingen. Similarly, compared to Göttingen, the respective increase in Svalöv were 26, 73, 313 and 377 fold in cultivars Oase, Express, Laser and Falcon, respectively. When the two locations with relatively higher disease infestation were compared with each other, a 4 (in Oase), 2 (in Express), 142 (in Laser) and 7 (in Falcon) fold amount of VL DNA was quantified in Svalöv compared to Fehmarn. Furthermore, despite very low levels of infestations across all locations at earlier growth stages (BBCH65 and $\mathrm{BBCH} 70$ ), qPCR results still showed a relatively higher fungal biomass in Fehmarn and Svalöv than in Göttingen (Fig. 2.6). The above described local variations in VL disease infestations was consistent with disease index assessment results of previous studies conducted with other sets of oilseed rape genotypes (Eynck, 2008; Knüfer, 2013).
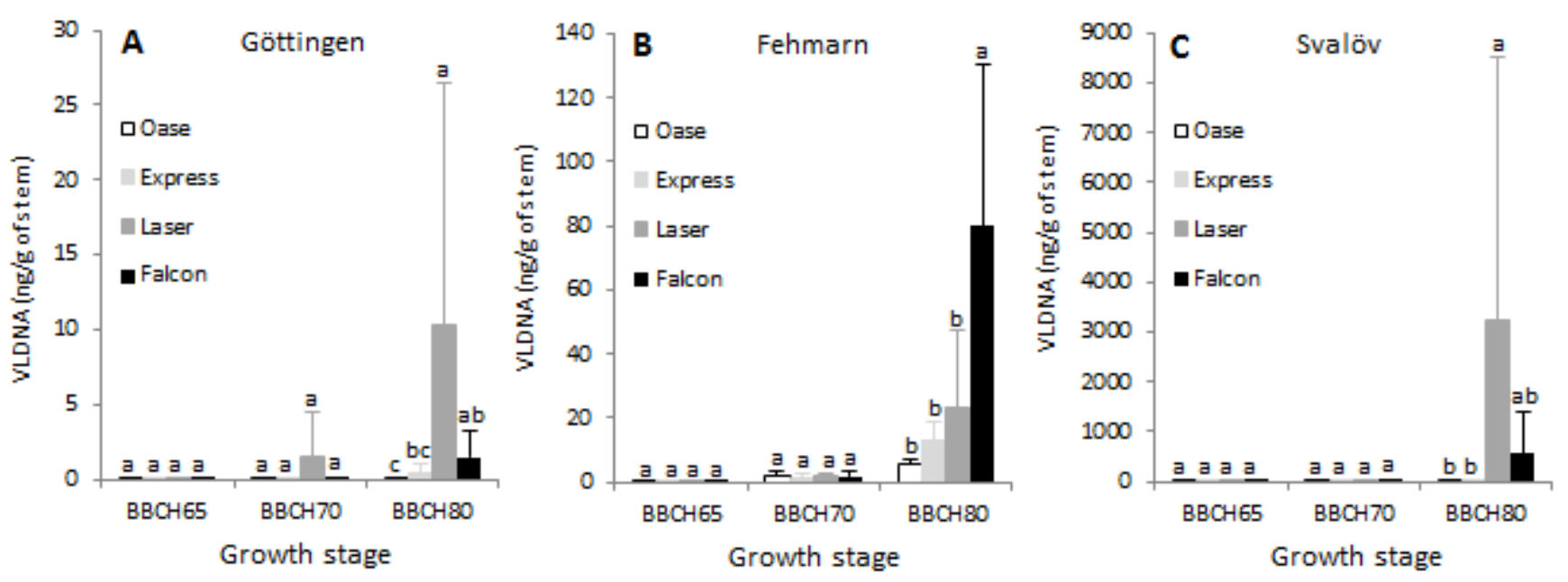

Figure 2.6 qPCR analysis of Verticillium longisporum infection at different developmental stages of resistant and susceptible winter oilseed rape (Brassica napus) varieties grown under field conditions in 2012/13. Mean data obtained from four biological replicates of a DNA sample extracted from 10 pooled stems per replicate is shown. Bars represent standard deviations of biological replicates. For each developmental stage, means followed by the same letter are not significantly different at $\mathrm{p}=0.05$. VLDNA, Verticillium longisporum DNA.

One of the possible explanations for the locational differences in VL disease levels could be the presence of variable climatic and soil conditions (Table 2.2). The amount of inoculum in the soil which is positively associated with long tradition of intensive oilseed rape cultivation in Svalöv (Johansson et al., 2006) and Fehmarn (Eynck, 2008) might be also among the most determining factors that contributed for the high levels of infestation in these locations. Also, the contribution of possible variations in composition, diversity or abundance of aggressive $V$. longisporum isolates among the different locations should not be ignored or underestimated. In general, the information gathered from stubble disease index assessment seems insufficient particularly with regard to immediate decision making on the use of possible control measures. This is mainly because plants at this stage have already attained the maximum stage of development and taking any action at this time point may not be economical. 
Nevertheless, determining disease levels on stubble samples provide reliable information about the amount of VL microsclerotia that join the soil bank and this is the major input required for forecasting disease epidemics. This intern helps early decision-making regarding choice of control options such as selection of resistant varieties, crop rotation schemes and possible use of pre emergence fungicides.

\subsubsection{Outdoor experiment}

As described previously, the major objective of this experiment was to find out the causes of variations between field and greenhouse $V$. longisporium symptoms in oilseed rape (particularly stunting and excessive branching). The study was conducted based on the assumption that differences in climatic factors, type of inoculum or inoculation methods are among the major factors contributing for the above mentioned variations in symptoms. To investigate possible effects of these factors, the outdoor experiment was conducted using the same type of inoculum and an identical inoculation method as in the greenhouse experiments. To mimic field conditions, inoculated plants were transplanted to pots and grown in outdoor conditions where they experienced similar climatic conditions as field-grown plants. Details of the methodologies are found in section 2.2.3.2 and 2.2.3.3.
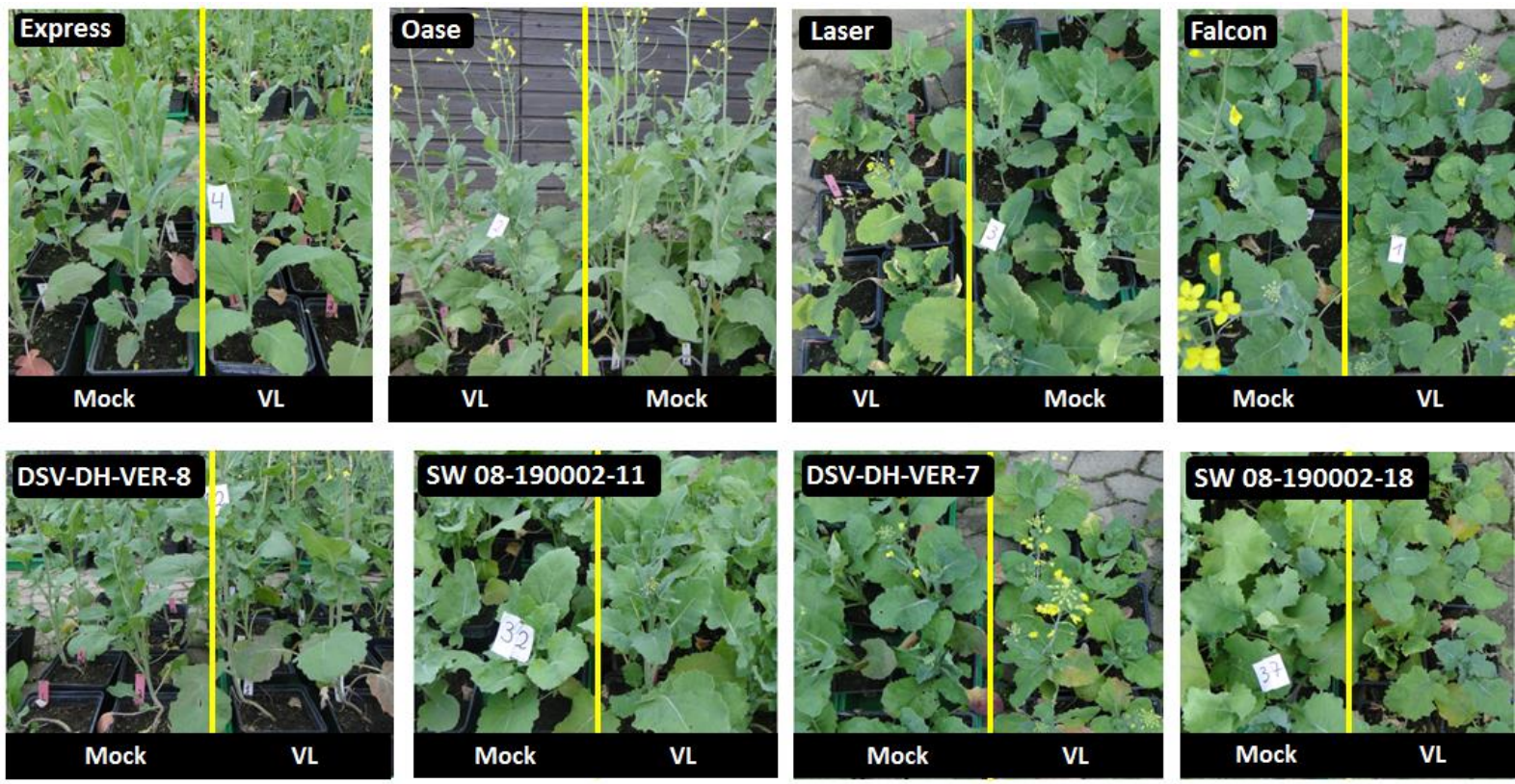

Figure 2.7 Phenotypes of mock and VL infected Brassica napus reference varieties (Top) and representative double haploid lines (bottom) showing resistance and susceptible responses towards Verticillium longisporum infection in the outdoor experiment at 49DPI. Inoculation of vernalized seedlings (BBCH14) was performed following a standard cut-root inoculation method. Mock, control plants infected with sterile water. VL, infected with Verticillium longisporum. Express and Oase are resistant reference varieties. Laser and Falcon were used as susceptible references. DH lines DSV-DH-VER-8 and SW08-190002-11 indicate resistance response while lines DSVDH-VER-7 and SW-08-190002-18 show susceptible reactions. Labels shown in white tags in each genotype are plot numbers. 
Disease assessment (net AUDPC) results showed considerable differences between susceptible and resistant reference varieties. The remaining $36 \mathrm{DH}$ lines tested in this experiment also showed a wide range of performances (Fig. 2.8A). Phenotypes of reference varieties and four lines representing susceptible and resistant individuals of a DH population are shown in Fig. 2.7. Except for few lines, severe stunting was observed in most genotypes that had high net AUDPC values (Fig. 2.8B).
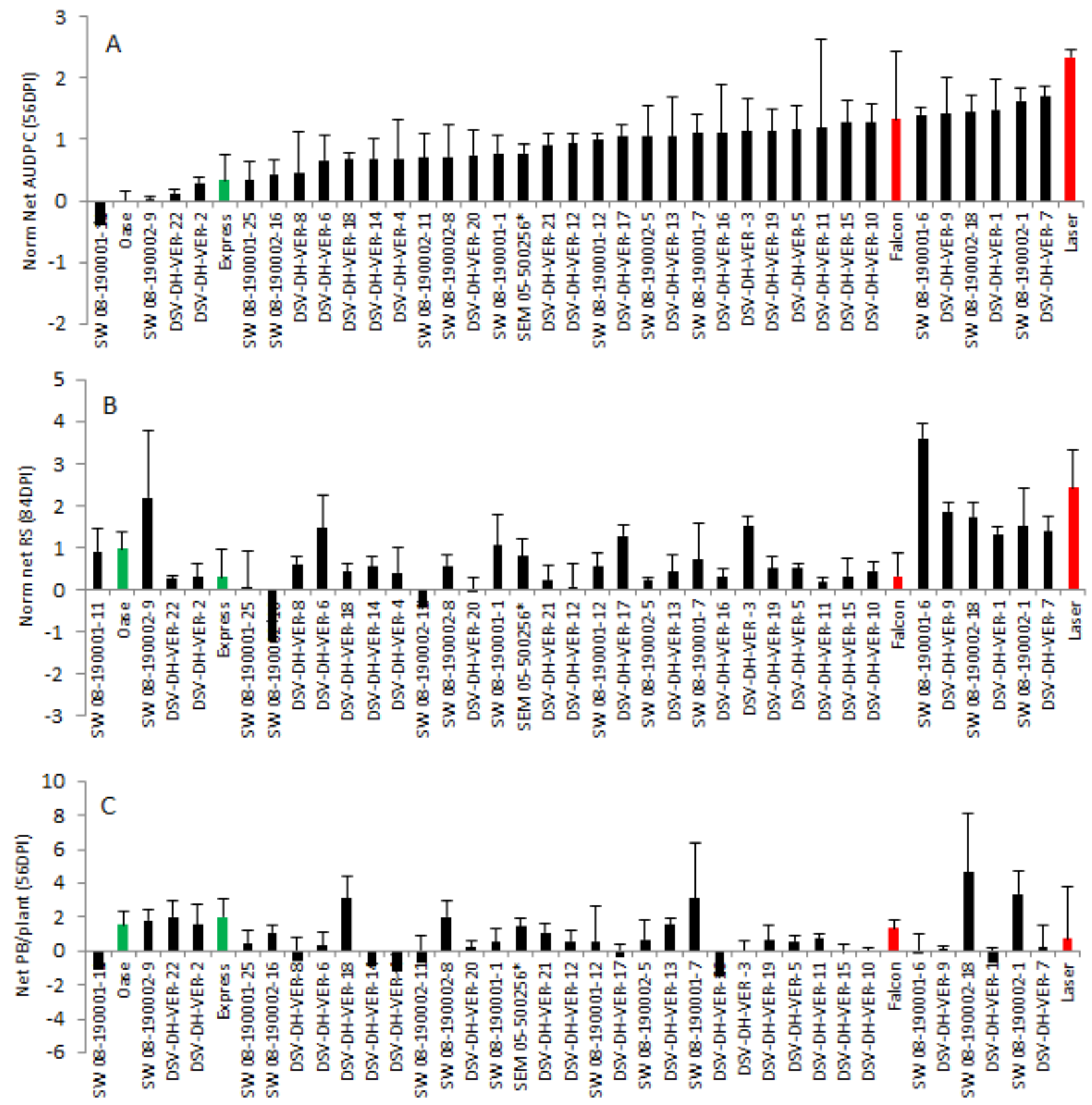

Brassica napus lines

Figure 2.8 Normalized net AUDPC (A), Normalized net relative stunting (B) and net primary branches per plant (C) measured from Brassica napus double haploid lines screened for Verticillium longisporum resistance in the outdoor experiment at 56DPI. AUDPC, Area under disease progress values. RS, relative stunting. PB, primary branches. Green and red bars represent values of resistant and susceptible controls, respectively. ${ }^{*}$, not a double haploid line. For all parameters mean \pm standard deviation data obtained from three biological replicates is shown. Genotypes are sorted according to increasing net AUDPC values. 
With regard to the branching phenotype, it was found that upon infection with VL, some lines (37.5\% of the total) produced at least one additional primary branch per plant compared to uninfected plants. On the other hand, in a few other $\mathrm{DH}$ lines ( $7.5 \%$ of the total), infection reduced branching. For a considerable proportion (55\% of the total), VL infection had no effect at all (Fig. 2.8C). This result suggests that the characteristic excessive branching usually observed in infected plants is not exclusively related to either susceptible or resistant reactions of OSR to VL.

Table 2.12 Correlation between outdoor performance vs field and greenhouse screening results of Brassica napus double haploid lines evaluated for resistance against Verticillium longisporum.

\begin{tabular}{ccccccccccccc}
\hline & \multicolumn{4}{c}{ Fehmarn (2012) } & \multicolumn{3}{c}{ Svalov (2012) } & \multicolumn{3}{c}{ Greenhouse } & \multicolumn{3}{c}{ Outdoor } \\
& SDI & RDI & VLDNA & SDI & RDI & VLDNA & NNA & NRS & NGS & NPBPP & NRS \\
\hline OD NNA & $-0.15^{\text {ns }}$ & $-0.21^{\text {ns }}$ & $\mathbf{0 . 3 7 ^ { * }}$ & $-0.016^{\text {ns }}$ & $-0.01^{\text {ns }}$ & $0.24^{\text {ns }}$ & $-0.08^{\text {ns }}$ & $0.18^{\text {ns }}$ & $-0.03^{\text {ns }}$ & $0.01^{\text {ns }}$ & $\mathbf{0 . 3 8 ^ { * }}$ \\
OD NRS & $-0.07^{\text {ns }}$ & $0.047^{\text {ns }}$ & $0.18^{\text {ns }}$ & $0.09^{\text {ns }}$ & $-0.01^{\text {ns }}$ & $0.04^{\text {ns }}$ & $0.09^{\text {ns }}$ & $0.32^{\text {ns }}$ & $-0.37^{*}$ & $0.06^{\text {ns }}$ & \\
OD PBPP & $-0.09^{\text {ns }}$ & $-0.13^{\text {ns }}$ & $0.01^{\text {ns }}$ & $-0.12^{\text {ns }}$ & $-0.13^{\text {ns }}$ & $-0.03^{\text {ns }}$ & $0.11^{\text {ns }}$ & $0.05^{\text {ns }}$ & $-0.18^{\text {ns }}$ & & \\
OD NGS & $-0.08^{\text {ns }}$ & $-0.26^{\text {ns }}$ & $-0.14^{\text {ns }}$ & $-0.19^{\text {ns }}$ & $-0.19^{\text {ns }}$ & $-0.08^{\text {ns }}$ & $-0.24^{\text {ns }}$ & $-0.18^{\text {ns }}$ & & & & \\
\hline
\end{tabular}

Pearson correlation analysis was performed using product-moment procedure of STATISTICA (Version 10 StatSoft, inc. 2011). Data obtained from 36 lines and 4 reference varieties were used. Minus signs indicate negative correlations. Significant correlations are indicated in bold font. ${ }^{*}$, significant at $\mathrm{P}=0.05$. ${ }^{* *}$, significant at $\mathrm{P}=0.01$. $* * *$, significant at $P=0.001$. ns, not significant. GH, greenhouse experiment. OD, outdoor experiment. SDI, field shoot disease index. RDI, field root disease index. VLDNA, Verticillium longisporum DNA in hypocotyl. NNA, net AUDPC. NRS, normalized relative stunting. NPBPP, net primary branches per plant. NGS, net growth stage.

The above observations were strongly supported by results of correlation analyses (Table 2.12). Stunting was significantly and positively correlated with net AUDPC $(r=0.38)$. Despite the potential impact of climatic and soil factors, inoculation of plants by soaking cut-roots in spore suspensions might induce strong and rapid infections which may result in early changes in internal plant metabolism (such as hormonal imbalance) leading to stunted growth. Under field conditions however, since the major sources of inoculum are dormant resting structures (microsclerotia), it might take longer until the fungus receives a host signal, initiates germination, starts infection and finally spreads into the shoot. This time gap may provide the host plant a sufficient time to attain the maximum possible plant height and that is probably why VL is not causing stunting symptoms in the field. The negative significant correlation ( $r=-0.37)$ found between stunting and plant developmental growth stage also strongly supports the above hypothesis. Thus, compared to fast growing plants, genotypes with slow growth rates might be more vulnerable to stunting caused by VL infection. Branching did not show any correlation with any of the field, greenhouse or outdoor disease evaluation parameters (Table 2.12). This indicates that increased branching in OSR due VL infection is genotype dependent and not related 
to susceptibility to $V$. longisporum. Another interesting relationship was the strong and significant correlation found between field QPCR and outdoor net AUDPC $(r=37)$, suggesting the reliability and consistency of the GPCR method for assessment of VL resistance in oilseed rape under various experimental or environmental conditions.

Further effects of VL infection on plant growth rate were assessed by evaluating developmental growth stages of mock and VL-infected plants. The mean growth stage of all genotypes at the last time point of disease index assessment (56DPI) is shown in Fig. 2.9. For most genotypes, differences in growth stages of infected and control plants were not exaggerated. Furthermore, except for the only correlation with outdoor NRS, this parameter showed no significant relationship with all the other disease evaluation results from greenhouse, field and outdoor experiments (Table 2.12). Nevertheless, it was interesting that correlation of growth stage with all other disease parameters (be it significant or not) had negative coefficients, which might suggest a growth retarding effect of VL.

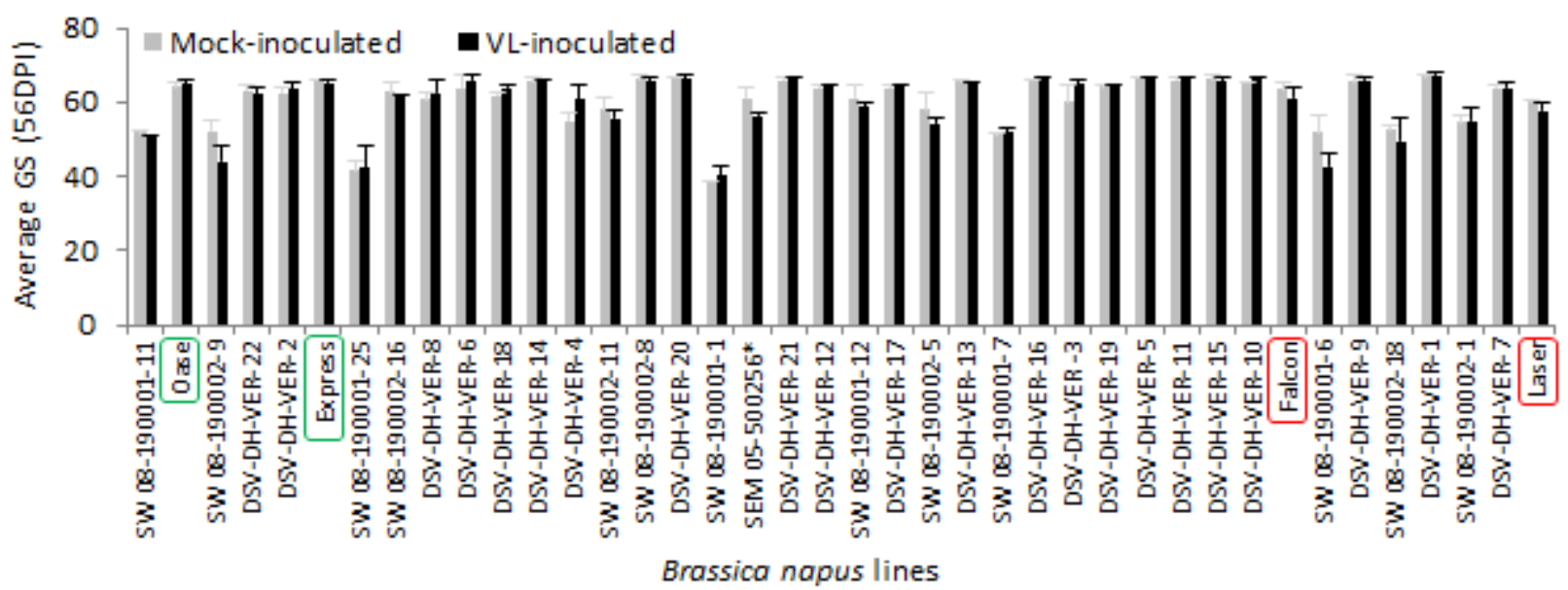

Figure 2.9 Average growth stage of Brassica napus double haploid lines and reference varieties screened for Verticillium longisporum resistance in the outdoor experiment. Inoculation of vernalized seedlings (BBCH14) was performed following a standard cut-root inoculation method. Resistant and susceptible controls are indicated in green and red marks, respectively. ${ }^{*}$, not a double haploid line. GS, growth stage according to BBCH scale. Mean tstandard deviation data obtained from three biological replicates is shown. Genotypes are sorted according to increasing net AUDPC values. 


\subsection{References}

Berg G., Frankowski J. and Bahl H. 1999. Biocontrol of verticillium wilt in oilseed rape by chitinolytic Serratia Plymuthica. Proceedings of $10^{\text {th }}$ International Rapeseed Congress. Canberra, Australia.

Carré P. and Pouzet A. 2014. Rapeseed market, worldwide and in Europe. Oilseeds and fats crops lipids, 21 (1) D102.

Dunker S., Keunecke H., Steinbach P., von Tiedemann A. 2008. Impact of Verticillium longisporum on yield and morphology of winter oilseed rape (Brassica napus) in relation to systemic spread in the plant. Journal of Phytopathology 156: 698-707.

Eynck C. 2008. Identification of resistance sources and characterization of resistance factors in Brassica species to Verticillium longisporum. PhD thesis. Georg-August-Universität Göttingen, Göttingen, Germany.

Eynck C., Koopmann B. and von Tiedemann A. 2009a. Identification of Brassica accessions with enhanced resistance to Verticillium longisporum under controlled and field conditions. Journal of Plant Diseases and Protection, 116(2), 63-72.

Farkas Á. and Zajácz E. 2007. Nectar production for the hungarian honey industry. The European Journal of Plant Science and Biotechnology, 1(2):125-151.

França S. C., Spiessens K., Pollet S., Debode J., De Rooster L., Callens D. and Höfte M. 2013. Population dynamics of Verticillium species in cauliflower fields: Influence of crop rotation, debris removal and ryegrass incorporation. Crop Protection, 54:134-141.

Gladders P. 2009. Relevance of Verticillium wilt (Verticillium longisporum) in winter oilseed rape in the UK. HCGA Research Review, 72:1-39.

Goicoechea N. 2009. To what extent are soil amendments useful to control Verticillium wilt? Pest Management Science, 65:831-839.

Happstadius I., Ljungberg A., Kristiansson B. and Dixelius C. 2003. Identification of Brassica oleracea germplasm with improved resistance to Verticillium wilt. Plant Breeding, 122:30-34

Inderbitzin P., Davis R. M., Bostock R. M. and Subbarao K. V. 2011a. The Ascomycete Verticillium longisporum is a hybrid and a plant pathogen with an expanded host range. PLOS ONE, 6(3): e18260.

Karapapa V. K., Bainbridge B. W. and Heale J. B. 1997. Morphological and molecular characterization of Verticillium longisporum comb. nov., pathogenic to oilseed rape. Mycological Research, 101:1281-1294.

Klosterman S. J., Atallah Z. K., Vallad G. E. and Subbarao K. V. 2009. Diversity, pathogenicity, and management of Verticillium species. Annual Review of Phytopathology. 47:39-62. 
Knüfer J. 2013. Improvement of winter oilseed rape resistance to Verticillium longisporum- Assessment of field resistance and characterization of ultrastructural plant responses. PhD thesis. GeorgAugust-Universität Göttingen, Göttingen, Germany.

Leino M. 2006. Fungal diseases on oilseed rape and turnip rape. Norrköping, Sweden.

Orlovius K. 2003. Oilseed rape. In: Kirbky, E. A. (Ed.).Fertilizing for high yield and quality, Bulletin 16. International Potash Institute. Basel, Switzerland.

Rygulla W., Friedt W., Seyis F., Lühs W., Eynck C., von Tiedemann A. and R. J. Snowdon. 2007a. Combination of resistance to Verticillium longisporum from zero erucic acid Brassica oleracea and oilseed Brassica rapa genotypes in resynthesized rapeseed (Brassica napus) lines. Plant Breeding, 126:596-602.

Rygulla W., Snowdon R. J., Eynck C., Koopmann B., von Tiedemann A., Lühs W. and Friedt W. $2007 \mathrm{~b}$. Broadening the genetic basis of Verticillium longisporum resistance in Brassica napus by interspecific hybridization. Phytopathology, 97:1391-1396.

Sattell R., Dick R., Ingham R., Karow R., Kaufman D. and D. McGrath. 1998. Rapeseed (Brassica campestris/Brassica napus). Oregon State University, USA.

Zeise K. and von Tiedemann A. 2002. Host specialization among vegetative compatibility groups of Verticillium dahliae in relation to Verticillium longisporum. Journal of Phytopathology, 150, 112119. 
3. Searching for cultivar-related resistance factors to Verticillium longisporum in oilseed rape (Brassica napus) xylem sap*

*This chapter have been submitted for publication to Phytopathology. Manuscript ID PHYTO-07-160281-R: Lopisso T. D., Knüfer J., Koopmann B. and von Tiedemann A. Growth of Verticillium longisporum in Brassica napus xylem sap is independent from cultivar resistance but promoted by plant ageing.

Department of Crop Sciences, Division of Plant Pathology and Crop Protection, Georg August University, Grisebachstr. 6, 37077 Göttingen, Germany; ${ }^{2}$ Current address: Strube Research GmbH \& Co. KG, 38387 Söllingen, Germany

\subsection{Introduction}

Verticillium species are among the most important fungal pathogens and cause vascular diseases in several economically important crop species. Among these, the recently evolved species Verticillium longisporum (Inderbitzin et al., 2011a) is becoming a potential threat in oilseed rape (OSR) production particularly in the Northern European regions (Heale and Karapapa, 1999) where production of this crop is particularly widespread (Carré and Pouzet, 2014). One of the most peculiar characteristics of $V$. longisporum (VL) which makes it different from other Verticillium species is its host specificity towards members of the Brassicaceae including OSR (Karapapa et al., 1997; Zeise and Tiedemann, 2002). In addition, despite effective colonisation and successful spread throughout the plant vascular system, VL does not induce wilting. Typical symptoms are rather leaf chlorosis, stunted growth and premature senescence and ripening (Karapapa et al., 1997; Leino, 2006) potentially resulting in substantial yield losses particularly under conditions of high disease incidence (Dunker et al., 2008). Systemic infection and long term soil survival of this pathogen significantly limit the options available to manage this disease in OSR with fungicides. Hence, besides preventive measures such as crop rotation, improvement of cultivar resistance is the sole potential control strategy. Accordingly, a range of studies from identification of resistance sources (Happstadius et al., 2003; Rygulla et al., 2007a; Rygulla et al., 2007b, Rygulla et al., 2008) to field performance evaluation experiments (Eynck et al., 2009a) have been undertaken in the past. These studies have been successful in identifying promising lines from which resistance traits can be derived. Nevertheless, there is lack of knowledge regarding the mechanisms of resistance and this can potentially hamper the effort of introgression of VL resistance traits into commercial OSR varieties. 
Plant defence to vascular plant pathogens can generally consist of physical or chemical defence responses. The most common physical defence mechanisms that prevent or contain vertical and lateral xylem spread of vascular pathogens include formation of tyloses accompanied with accumulation of gels and gums, vascular coating, callose deposition, xylem wall swelling and vascular HR (Hypersensitive response). In addition, chemical defence compounds accumulating in the xylem and killing or inhibiting fungal growth are common strategies. Among these, phenols, phytoalexins and several proteins and enzymes play a major role (Yadeta and Thomma et al., 2013).

Several potential mechanisms of VL resistance in OSR have been proposed. Eynck et al. (2009b) was the first to reveal the involvement of vascular occlusions, phenolics and lignin in OSR resistance against VL. Later on, other studies have shown the crucial role of phenylpropanoids (Obermeier et al., 2013; Kamble et al., 2013). Singh et al., (2012) demonstrated that OSR xylem sap is capable of inhibiting the in vitro growth of $V L$ and suggested a possible role of pre-existing xylem sap proteins in defence. Despite the fact that VL infection caused no change in total xylem sap protein content, xylem sap extracted from susceptible infected B. napus plants significantly reduced in vitro growth of VL (Floerl et al., 2008). Another study aiming at the general analysis of xylem sap proteins in B. napus identified more than 60 different proteins, some of which were associated with plant defence against pathogens (Kehr et al., 2005). In a study on the interaction of $B$. napus with VL, an enhanced accumulation of defence related antifungal proteins (including endochitinase, peroxidase PR-proteins and glucanase) were identified in the leaf apoplastic washing fluids of VL-susceptible B. napus (Floerl et al. (2008). In contrast, an expression analysis of the Salicylic acid (SA) signalling pathway marker gene PR1 by Kamble et al. (2013) showed no role of this gene in $B$. napus resistance against VL. In another study focusing on plant hormones involved in resistance signalling pathways, VL infection caused an increased accumulation of SA in the xylem sap or in hypocotyl tissue extract of OSR but had no effect on jasmonic acid (JA) and abscisic acid (ABA) concentrations (Ratzinger et al., 2009; Kamble et al., 2013). Johansson et al. (2006) reported that VL-resistance in Arabidopsis is regulated by JA and ethylene associated signals, but not by SA. According to Veronese et al. (2003), impairment of JA and SA signalling caused no sensitivity of Arabidopsis plants to Verticillium dahliae.

The complex changes occurring in xylem tissue of $B$. napus as a consequence of VL infection is a clear indication for the hypocotyl tissue to be the key site of crucial processes regulating the host-pathogen interaction. According to previous field and greenhouse resistance evaluation studies, it is clear that there is significant variation in the level of VL-resistance among B. napus lines. Moreover, distinct differences between resistant and susceptible genotypes with regard to the rate and intensity of 
physical resistance factors as well as tissue extract chemical responses are known. However, except for a limited number of reports on the existence of xylem sap compounds involved in general resistance responses, nothing is known about the presence of cultivar-related VL- resistance factors in the xylem sap of $B$. napus. In the past, most reports focusing on VL-resistance factors in B. napus or Arabidopsis xylem sap were based on only a single mainly susceptible genotype. Moreover, as mentioned above, there are conflicting reports on the role or involvement of plant hormones and metabolites in VL resistance of $B$. napus and Arabidopsis. As a result, concrete and comprehensive information regarding the existence and role of cultivar-related VL-resistance factors in the xylem sap of OSR is not available. The present study therefore aims to compare the composition and growth inhibitory effect of xylem sap extract from susceptible and resistant OSR genotypes and to explore whether xylem sap play a major role in cultivar-related resistance of OSR against $V$. longisporum.

\subsection{Materials and methods}

\subsubsection{Treatments and experimental design}

The overall study was carried out in two independent experiments each consisting of greenhouse trials and in vitro bioassays. In the first experiment in the greenhouse, a completely randomized design with four replications was chosen. Two OSR genotypes (VL-resistant SEM 05-500256 and VL-susceptible Falcon) and two inoculation factors (mock and VL-inoculated) resulted in four treatments (Table 3.1). For the phenotypic disease evaluation, 20 plants per treatment (5 plants per replication) were used. For xylem sap extraction at two time points ( 21 and 28 days post inoculation, DPI), at least 45 extra plants per treatment were included, since this was a destructive sampling. The in vitro bioassay consisted of 10 treatments derived from the combination of genotype, disease and xylem sap filtration (Table 3.2), and was conducted in 96-well microtitre plates. In the greenhouse experiment, mock-inoculated plants were used as control whereas in the in vitro bioassay, two controls, sterile distilled water with conidial suspension and sterile distilled water alone, represented the control treatments (Table 3.1 and 3.2). The second experiment was conducted in a climate chamber with the additional objective to investigate VLresistance factors in xylem sap of plants at different stages of ageing. In this study, a different VLresistant B. napus genotype (Aviso) was used (Table 3.1) and xylem sap samples were collected in 2-3 day intervals (From 13 to $28 \mathrm{DPI}$ ). All other conditions and settings of the climate chamber and bioassay experiments were similar as described above.

\subsubsection{Plant material and cultivation}

Three B. napus genotypes were used. Seeds for the VL-susceptible cultivar Falcon were purchased from NPZ (Norddeutsche Pflanzenzucht, Hans-Georg Lembke KG, Hohenlieth, Germany). Seeds for the VL- 
tolerant genotypes SEM and Aviso were obtained from Lantmännen SW Seed (Svalöv, Sweden) and Danisco Seed (Sweden), respectively. Before sowing, seeds were double surface sterilized with $70 \%$ ethanol for 2 min under constant shaking and rinsed with autoclaved tap water. Seeds were grown on autoclaved silica sand in a climate-controlled chamber (day/night conditions of $14 \mathrm{~h} / 10 \mathrm{~h}$ at $23^{\circ} \mathrm{C}$ ) for two weeks and then inoculated with VL as described below.

Table 3.1 Treatment descriptions and designations for greenhouse/climate chamber experiments.

\begin{tabular}{cccc}
\hline No. & Designation & $\begin{array}{c}\text { Experimental factors and descriptions } \\
\text { Genotype }\end{array}$ & Disease \\
\hline 1 & SM & SEM 05-500256 & Mock-inoculated \\
2 & SVL & SEM 05-500256 & VL-inoculated \\
3 & FM & Falcon & Mock-inoculated \\
4 & FVL & Falcon & VL-inoculated \\
5 & AM & Aviso & Mock-inoculated \\
6 & AVL & Aviso & VL-inoculated
\end{tabular}

S, SEM 05-500256. F, Falcon. A, Aviso. M, mock-inoculated. VL, Verticillium longisporum inoculated.

Table 3.2 Treatment descriptions and designations for bioassay studies.

\begin{tabular}{ccccc}
\hline No. & Designation & \multicolumn{3}{c}{ Experimental factors and descriptions } \\
& & Genotype & Disease & Xylem sap filtration \\
\hline 1 & SM & SEM 05-500256 & Mock-inoculated & unfiltered \\
2 & SMf & SEM 05-500256 & Mock-inoculated & filtered \\
3 & SVL & SEM 05-500256 & VL-inoculated & unfiltered \\
4 & SVLf & SEM 05-500256 & VL-inoculated & filtered \\
5 & FM & Falcon & Mock-inoculated & unfiltered \\
6 & FMf & Falcon & Mock-inoculated & filtered \\
7 & FVL & Falcon & VL-inoculated & unfiltered \\
8 & FVLf & Falcon & VL-inoculated & filtered \\
9 & AMf & Aviso & Mock-inoculated & filtered \\
10 & AVLf & Aviso & VL-inoculated & filtered \\
11 & BDWVL & VL growth on sterile bi-distilled water & \\
12 & BDW & Sterile bi-distilled water alone & \\
\hline
\end{tabular}

S, SEM 05-500256. F, Falcon. A, Aviso. M,mock-inoculated. VL, Verticillium longisporum inoculated. f, filtered xylem sap, BDW, sterile bi-distilled water.

\subsubsection{Fungal growth and inoculation technique}

Verticillium longisporum isolate VL43 (Fig. 1.2), a highly virulent isolate on OSR (Zeise and Tiedemann, 2002) was grown on potato extract glucose broth (Carl Roth GmbH + Co. KG, Karlsruhe, Germany) for 10 
days at $23^{\circ} \mathrm{C}$ in the dark with constant shaking. A standard non-cut root dip inoculation technique was used. Briefly, roots of two weeks old seedlings (BBCH 12) were washed under a running tap water and submerged for $1 \mathrm{~h}$ in a conidial suspension with a density of $10^{6}$ conidia/ml. Mock-inoculated plants were treated in the same way with autoclaved tap water. Treated seedlings were transferred to plastic pots $(200 \mathrm{ml})$ filled with a 3:1 (v/v) mixture of commercial soil (Fruhstorfer Erde, Type ; pH 6.0; HawitaGruppe, Vechta, Germany) and sand substrate. Transplants were grown in the greenhouse/climate chamber under similar conditions as described above.

\subsubsection{Xylem sap extraction}

Xylem sap was extracted from the lower hypocotyl part of different aged plants using the pressure bomb technique (Scholander et al., 1965). Briefly, plants were uprooted and soil materials adhering to roots were washed off under running tap water. The shoot part was removed by cutting the stem above the hypocotyl. The remaining hypocotyl part with the roots was immediately inserted into the chamber of the Scholander bomb (Model 600; PMS Instrument Company; Albany, USA). Xylem sap was collected by gradually increasing the chamber pressure until xylem sap appears at the cut surface. In a repeated experiment, in order to collect a sufficient amount of xylem sap, extraction was done by cutting plants at the tip of hypocotyl and collecting the exuding xylem sap from the cutting end using hand held pipette. Each treatment had three biological replications. Aliquots of approximately $1 \mathrm{ml}$ of xylem sap were collected from 15 plants pooled together and considered as one replication. Xylem sap samples collected from control and infected plants were immediately stored at $-20^{\circ} \mathrm{C}$ until further use.

\subsubsection{Disease evaluation}

Disease severity evaluation was done in a weekly interval from 7 to 28 DPI using a nine class assessment key modified from Zeise, 1992 (Table 4.2). Disease severity recordings were used to calculate AUDPC values (Campbell \& Madden, 1990) from which Net AUDPC (AUDPC ${ }_{\text {net }}$ ) values were calculated by subtracting the AUDPC value of control treatments from that of the values of inoculated treatments. That is $\mathrm{AUDPC}_{\text {net }}=\mathrm{AUDPC}_{\text {inoc }}-\mathrm{AUDPC}_{\text {mock }}$ where $\mathrm{AUDPC}_{\text {net }}, \mathrm{AUDPC}_{\text {inoc }}$ and $\mathrm{AUDPC}_{\text {mock }}$ refer to net $A U D P C$, AUDPC of VL-inoculated treatments and AUDPC of mock-inoculated treatments, respectively (Eynck et $a l, 2009 b$ ). Stunting was measured by recording plant height (from base of hypocotyl to the tip of the central main stem) at $28 \mathrm{DPI}$. At the same time, stem diameter (at the tip of hypocotyl) was precisely measured using a digital calliper. Quantification of fungal DNA by quantitative real time PCR was performed following the method described in 4.2.7.3. Cultivar's response to VL-infection was further determined by comparing the above hypocotyl biomass determined after oven drying $\left(70^{\circ} \mathrm{C}\right.$ for $\left.24 \mathrm{~h}\right)$. 


\subsubsection{In vitro bioassay}

Verticillium longisporum isolate VL43 was grown on potato extract glucose broth potato extract glucose broth (PEGB) (Carl Roth GmbH + Co. KG, Karlsruhe, Germany) as described in section 2.2.4. To remove mycelial masses, the culture was filtered with sterile cheesecloth. From the filtered culture, conidia were harvested by centrifugation for $10 \mathrm{~min}$ at $1046 \mathrm{~g}$ Conidia were washed three times by resuspending in sterile distilled water and the final conidial density was adjusted to $5 \times 10^{3}$ conidia/ml using a haemocytometer. To eliminate any bacterial growth during in vitro assay, filter sterilized streptomycin (20ppm) was added to the final spore suspension. To prepare filtered xylem sap treatments, xylem sap was filtered through a $0.2 \mu \mathrm{m}$ sterile Anotop 10 plus disposable syringe filter with aluminum oxide membrane material (Whatman $\mathrm{GmbH}$; Dassel, Germany). Measurement of fungal growth in xylem sap was performed using the method of Broekaert et al., 1990. The assay was set up as follows. First, $50 \mu \mathrm{L}$ of VL spore suspension $\left(5 \times 10^{3}\right.$ conidia/ml) and $200 \mu$ l of xylem sap were loaded in 96 well microtitre plates in three technical replicates. Prior to incubation, spores were allowed to settle for $30 \mathrm{~min}$ and the initial absorbance was taken at 580 nm in a microplate spectrophotometer ( $\mu$ Quant, BioTek Instruments, Bad Friedrichshall, Germany). Plates were placed in a moist chamber and incubated at $23^{\circ} \mathrm{C}$ for seven days in the dark. Fungal growth was monitored daily by taking the mean absorbance value of 25 readings taken from different spots in a single well. Fungal growth at different DAl (days after incubation) was obtained by subtracting initial absorbance values (0 DAI) from the daily absorbance recordings.

\subsubsection{Total protein assay}

The total protein content in xylem sap was measured with the Bradford method (Bradford, 1976). A standard curve was developed using a series of bovine serum albumin (BSA) standards in the range of 600 to $100 \mu \mathrm{g} / \mathrm{ml}$. Then, $276 \mu \mathrm{l}$ of $1 \mathrm{X}$-Roti-Quant staining solution (Carl Roth GmbH + Co. KG; Karlsruhe, Germany) was mixed with $5 \mu$ l of BSA standard solutions, xylem sap or double distilled water (used as blank) and loaded into the microtitre plate wells in triplicates. After incubation for $5 \mathrm{~min}$ at room temperature, absorbance at $595 \mathrm{~nm}$ was measured using a microplate spectrophotometer ( $\mu$ Quant, BioTek Instruments, Bad Friedrichshall, Germany). Total protein content in xylem sap was estimated by referring to the standard curve produced from known concentrations of BSA.

\subsubsection{Quantification of xylem sap total carbohydrate content}

Xylem sap total carbohydrate $(\mathrm{CHO})$ content was determined following the microplate format phenolsulphuric acid protocol (Masuko et al., 2005). Briefly, aliquots of standard glucose (GIc) solutions were prepared in double distilled water. Then, $100 \mu \mathrm{l}$ of Glu standard solution or xylem sap samples were 
mixed with $300 \mu \mathrm{l}$ of concentrated sulphuric acid, the solution was immediately amended with $60 \mu \mathrm{l}$ of $5 \%$ phenol and incubated for $5 \mathrm{~min}$ at $90^{\circ} \mathrm{C}$ in a static water bath. Subsequently, the solution was cooled for $5 \mathrm{~min}$ in a water bath at room temperature and $230 \mu \mathrm{l}$ of the reaction was loaded into 96 -well plates in triplicates. Absorbance was measured at $490 \mathrm{~nm}$, the absorption maximum of most sugars including mannose, xylose, frucose, galactose and glucose (Masuko et al., 2005). To calculate xylem sap total CHO content, mean absorbance values of xylem sap samples were referred to the standard curve produced from known concentrations of Glc.

\subsubsection{Data analysis}

Each experiment was repeated at least twice. All statistical analyses were performed using STATISTICA version 10 data analysis software (StatSoft, inc. 2011). One way ANOVA with Fisher Least Significant Difference (LSD) test was used to analyse means obtained from four or three biological replicates of greenhouse and laboratory experiments, respectively. Differences were considered statistically significant when P-values were less than 0.05. Before analysis of variance, all data sets were tested for normal distribution using the Shapiro-Wilk test and whenever required, ANOVA was performed on transformed data (Gomez and Gomez, 1984). Relationships between fungal growth and xylem sap total protein or carbohydrate concentration were determined by Pearson (product-moment) correlation analysis.

\subsection{Results}

The overall study was carried out in two different experimental series each consisting of a greenhouse trial, an in vitro bioassay and biochemical analyses. Greenhouse and in vitro bioassay experiments were repeated at least twice and provided consistent results. Results obtained from a single experiment are presented as follows.

\subsubsection{Greenhouse experiments}

Response of the two $B$. napus genotypes to VL infection was examined under greenhouse conditions. In this part of the study, phenotypic and molecular disease parameters that evidently demonstrate resistant or susceptible reactions of OSR to VL infection were measured. Evaluation of disease severity, from which net AUDPC was generated, was performed on a weekly basis using a standard disease index key. In addition, quantification of VL-DNA in hypocotyl tissue, measurement of stem thickness and determination of stunting were carried out four weeks after inoculation. Results obtained from all disease evaluations clearly showed strong resistance and susceptible reactions of genotype SEM and Falcon, respectively. Four weeks after inoculation, infected SEM plants remained green, vigorous and 
healthy while diseased Falcon plants showed extremely stunted growth with shrivelled, yellow and black veined leaves, which are typical symptoms of VL infection (Fig. 3.1). Disease levels as measured by net AUDPC showed five times higher disease severity in cultivar Falcon compared to the resistant genotype SEM. Accordingly, average net AUDPC values at 28 DPI recorded from infected SEM and Falcon plants were 10.2 and 49.7, respectively (Fig. 3.2A). Similarly, quantification of VL DNA from hypocotyl tissue of infected plants by quantitative PCR showed a 74 fold accumulation of fungal biomass in Falcon (1,419.2ng/g) compared to SEM (19.3ng/g) (Fig. 3.2B).
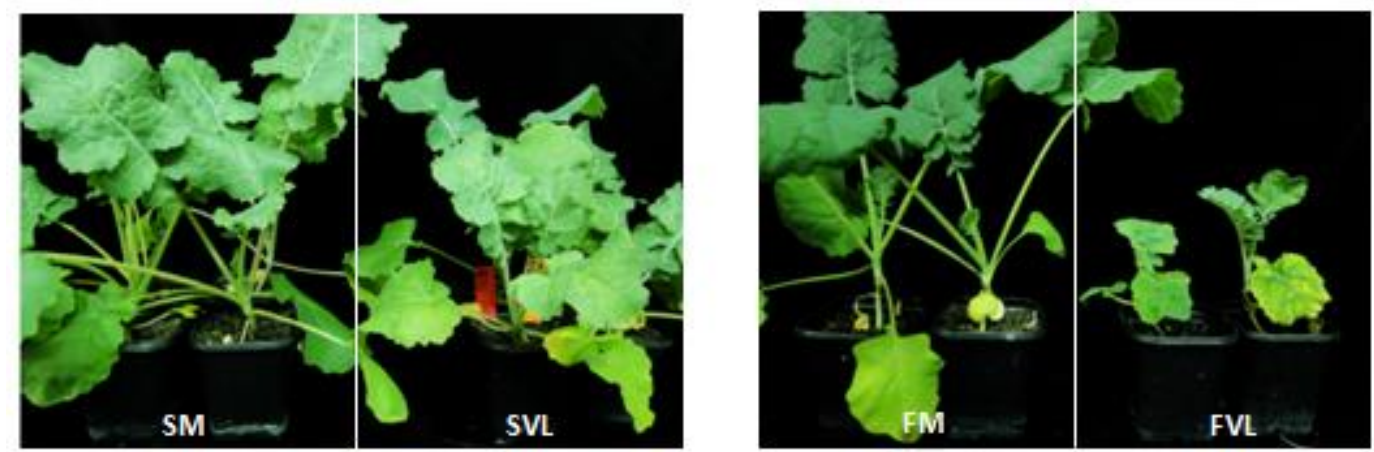

Figure 3.1 Phenotypes of resistant (left) and susceptible (right) Brassica napus plants 28 days after inoculation with Verticillium longisporum. Plants were inoculated by submerging non-cut roots of 10 day old seedlings in spore suspension $\left(10^{6} \mathrm{conidia} / \mathrm{ml}\right)$ for one hour. Mock-inoculated plants were similarly dipped in autoclaved tap water. SM, genotype SEM mock-inoculated. SVL, genotype SEM VL-inoculated. FM, cultivar Falcon mock-inoculated. FVL, cultivar Falcon VL-inoculated.
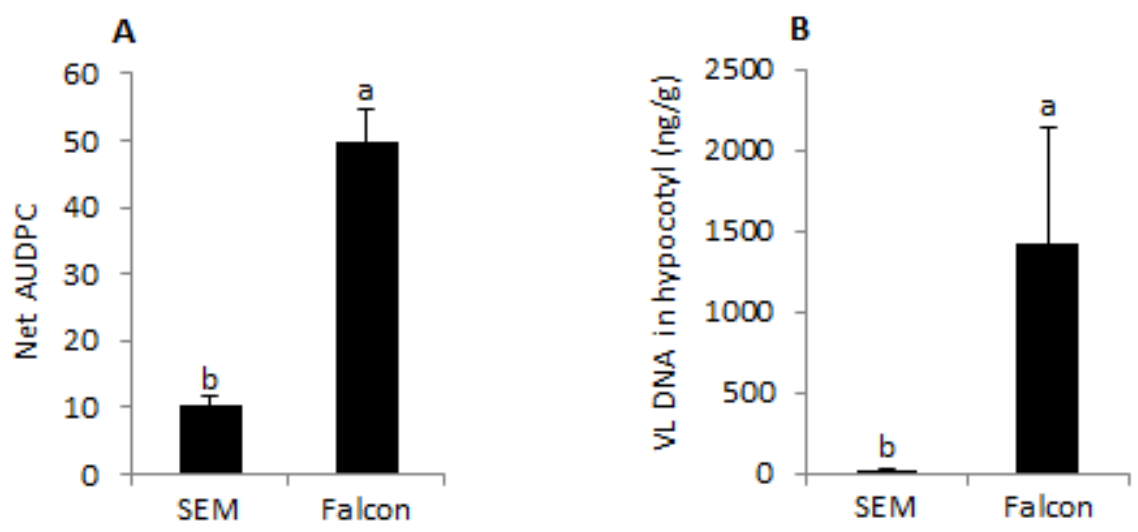

Figure 3.2 Evaluation of disease levels in resistant (SEM) and susceptible (Falcon) Brassica napus genotypes 28 days after inoculation with Verticillium longisporum. A, net area under disease progress curve values. B, quantification of Verticillium longisporum DNA in hypocotyl tissue by quantitative PCR. Bars indicate standard deviations of four biological replicates. Different letters on the bars indicate significant differences at $\mathrm{P} \leq 0.05$.

With regard to stem diameter, the two genotypes showed significant differences under both mock- and VL-inoculation conditions. In the absence of infection, the stem diameter of the VL-resistant genotype was significantly larger than the susceptible genotype. That means SEM had genetically wider stem thickness than Falcon. Four weeks after inoculation, infected SEM plants had a similar hypocotyl 
thickness $(3.8 \mathrm{~mm})$ as mock-inoculated plants. In cultivar Falcon however, infection significantly reduced hypocotyl thickness by $24 \%$ (Fig. 3.3A).

Regarding plant height, unlike the previously described disease parameters, VL infection caused significant effects both in resistant and susceptible plants. However, compared to SEM, the stunting effect of VL on the susceptible cultivar Falcon was significantly more severe. In this cultivar, reduction of plant height due to VL infection 28 days after inoculation was $69 \%$ while in genotype SEM, a relatively lower but still significant stunting (20\%) was recorded. As for stem diameter, the two genotypes had genetically different with regard to plant height where the VL-susceptible cultivar possessed significantly longer phenotype than the resistant genotype SEM (Fig. 3.3B). The responses of the resistant and susceptible genotypes to VL-infection were further shown in dry matter yield assessment. As expected dry matter yield in the susceptible genotype Falcon was significantly reduced by $68.6 \%$. In contrast, the reduction in SEM was not significant (Fig 3.3C). The resistance and susceptible responses of the two genotypes used in climate chamber experiments was also verified by disease index assessment (data not shown).
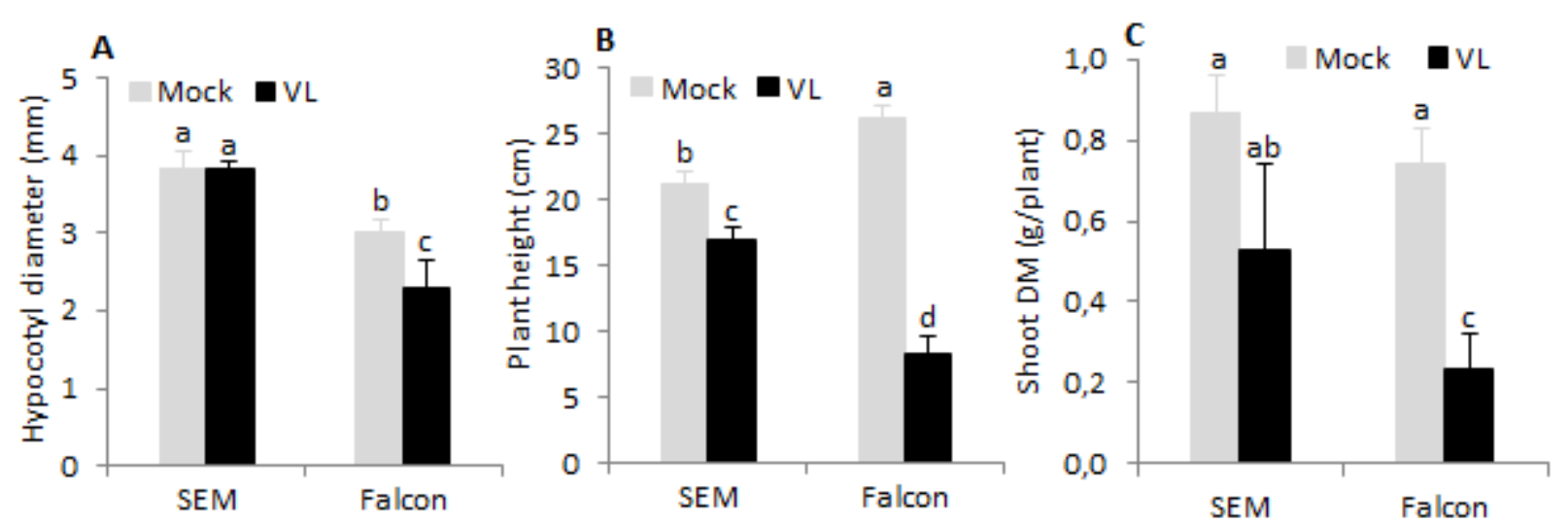

Figure 3.3 Assessment of stem thickness, plant height and shoot dry biomss in resistant (SEM) and susceptible (Falcon) Brassica napus genotypes infected with Verticillium longisporum. Measurement was done 28 days after inoculation. Bars indicate standard deviations of four biological replicates. Different letters on the bars indicate significant difference at $\mathrm{P} \leq 0.05$. Mock, control plants inoculated with sterile water. VL, Verticillium longisporum inoculated treatments. DM, dry matter.

\subsubsection{Analysis of Verticillium longisporum growth on Brassica napus xylem sap}

\subsubsection{Xylem sap bioassay}

To investigate the presence or absence of cultivar-specific xylem sap-residing VL resistance factors, growth of $V$. longisporum on xylem sap extracted from resistant and susceptible plants was analysed in vitro. With the intention of excluding the effect of xylem sap endophytes that might interfere with the bioassay and to further investigate the effect of xylem sap filtration on fungal growth, filtered xylem sap 
treatments were also included in this assay. The growth of VL on B. napus xylem sap was monitored on a daily basis using a spectrophotometer. Results showed that spore germination and further growth of VL begun after 2 to 3 days of incubation. Unlike the water control where no or only slight growth was detected, an enhanced and significantly higher level of fungal growth was observed in xylem sap medium from all treatment combinations. Analysis of variance showed that irrespective of VL infection or xylem sap filtration, the rate and level of VL growth in xylem sap extracted from resistant or susceptible plants was not significantly different (Fig. 3.4). Even after one week ofincubation (7 DAI), VL growth on filtered or unfiltered xylem sap extracts derived from either mock or VL-inoculated plants of both genotypes was similar (Fig. 3.5). Compared to filtered xylem sap however, a slightly higher growth of VL on unfiltered xylem sap of both genotypes was observed. Furthermore, again irrespective of the genotype, a slightly higher fungal growth on xylem sap extracted from VL-infected plants was noticed (Fig. 3.4; Fig. 3.5). Similar analyses of fungal growth measurements from 2 to 6 DAl showed no significant difference among all treatment combinations (Appendix 3.3).
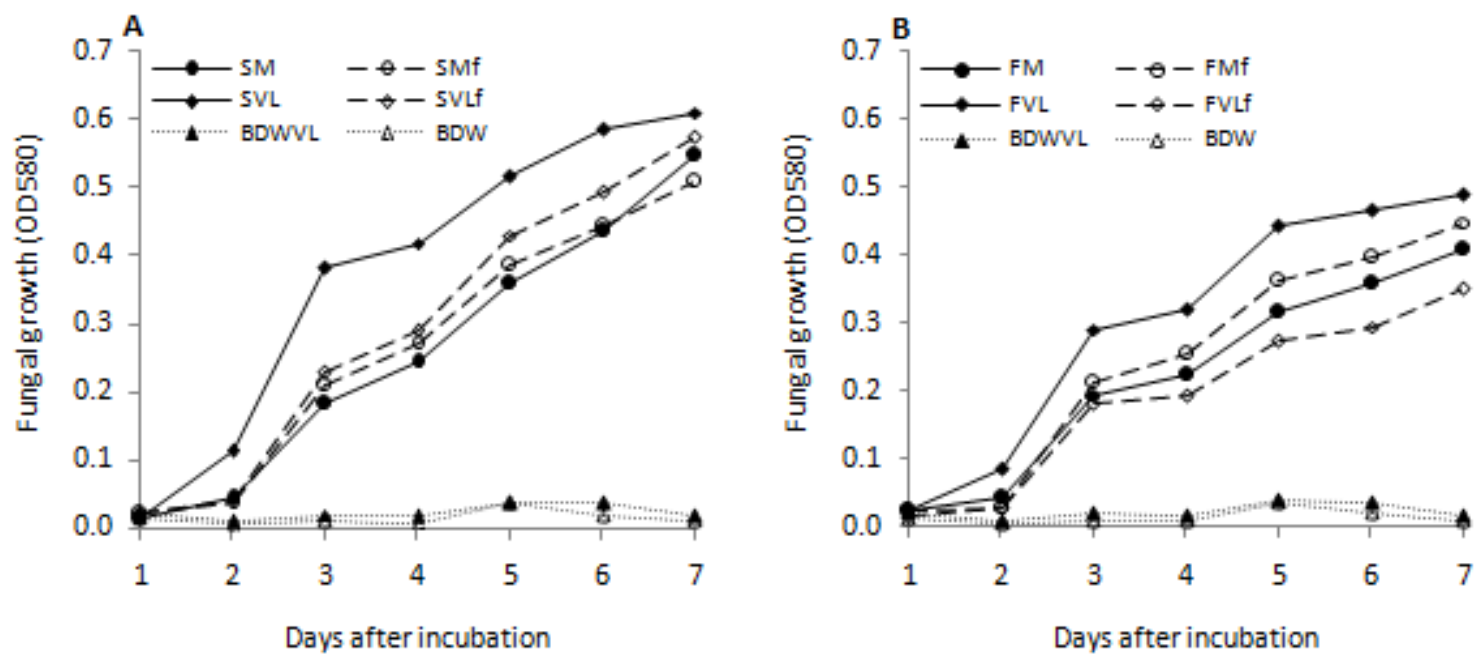

Figure 3.4 Spectrophotometric measurement of Verticillium longisporum growth on xylem sap extracted from resistant (SEM) and susceptible (Falcon) Brassica napus plants. Xylem sap was extracted 28 days after inoculation with Verticillium longisporum. A. Fungal growth in xylem sap extracted from genotype SEM. SM and SMf, respective fungal growth in unfiltered and filtered xylem sap extracted from mock-inoculated plants. SVL and SVLf, respective fungal growth in unfiltered and filtered xylem sap extracted from VL-inoculated plants. B. Fungal growth in xylem sap extracted from genotype Falcon. FM and FMf, respective fungal growth on unfiltered and filtered xylem sap extracted from mock-inoculated plants. FVL and FVLf, respective fungal growth in unfiltered and filtered xylem sap extracted from VL-inoculated plants. BDWVL, Fungal growth in sterile bi-distilled water. BDW, sterile bidistilled water without VL inoculum. At no DAl, there was any significant difference in fungal growth in the different xylem sap samples (Appendix 3.3).

\subsubsection{Xylem sap total protein content}

Protein analysis was performed in order to investigate the effect of VL infection on xylem sap protein concentration and to further find out whether changes in protein concentration are related to resistance 
or susceptible responses of $B$. napus genotypes to $V$. longisporum. Accordingly, the total soluble protein content of xylem sap samples used in fungal growth bioassays was analysed with the Bradford method. Results revealed the presence of considerable amounts of proteins in B. napus xylem sap. In mockinoculated plants, the quantities of total proteins in xylem sap of SEM and Falcon plants were 104.9 and $101.8 \mu \mathrm{g} / \mathrm{ml}$, respectively. When infected with $\mathrm{VL}$, a slightly higher $(109.4$ and $106.0 \mu \mathrm{g} / \mathrm{ml}$, respectively) but insignificant increase was found. Analysis of variance showed no significant difference in total soluble protein concentration of xylem sap samples extracted from resistant and susceptible genotypes. It was also found that VL infection and xylem sap filtration did not significantly alter protein concentrations. Nevertheless, a slight increase of total protein concentration due to VL infection and a reduction due to filtration was noticed in both genotypes (Table 3.3).

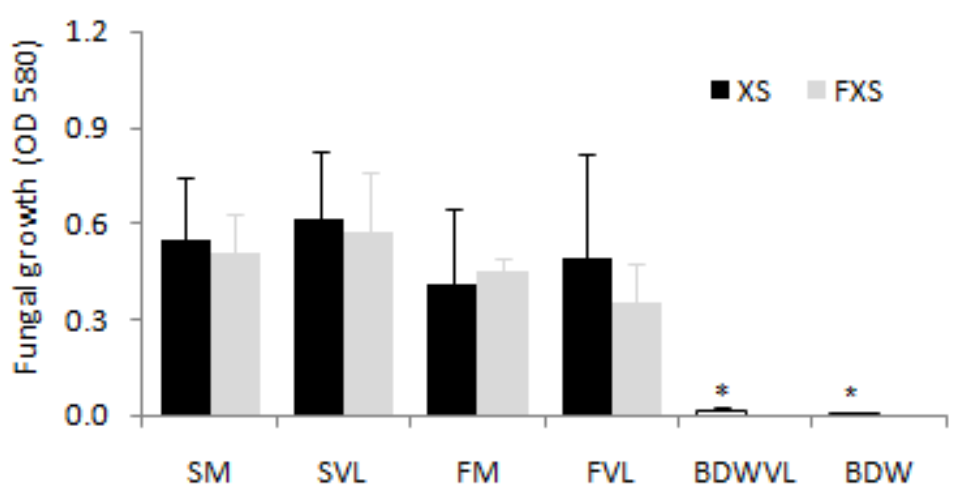

Figure 3.5 Spectrophotometric measurement of Verticillium longisporum growth seven days after incubation in xylem sap extracted from resistant (SEM) and susceptible (Falcon) Brassica napus plants. Xylem sap was extracted 28 days after inoculation. FXS, filtered xylem sap, XS, unfiltered xylem sap. SM, Growth of $V$. longisporum in xylem sap extracted from mock-inoculated SEM plants. SVL, fungal growth in xylem sap extracted from VL-inoculated SEM plants. FM, fungal growth in xylem sap extracted from mock-inoculated Falcon plants. FVL, fungal growth in xylem sap extracted from VL-inoculated Falcon plants. BDWVL, fungal growth on sterile bi-distilled water. BDW, sterile bi-distilled water without VL inoculum. *, significantly different at $\mathrm{P}=0.05$.

Table 3.3 Concentrations of total soluble protein in xylem sap of resistant (SEM) and susceptible (Falcon)

Brassica napus genotypes 28 days after inoculation with Verticillium longisporum.

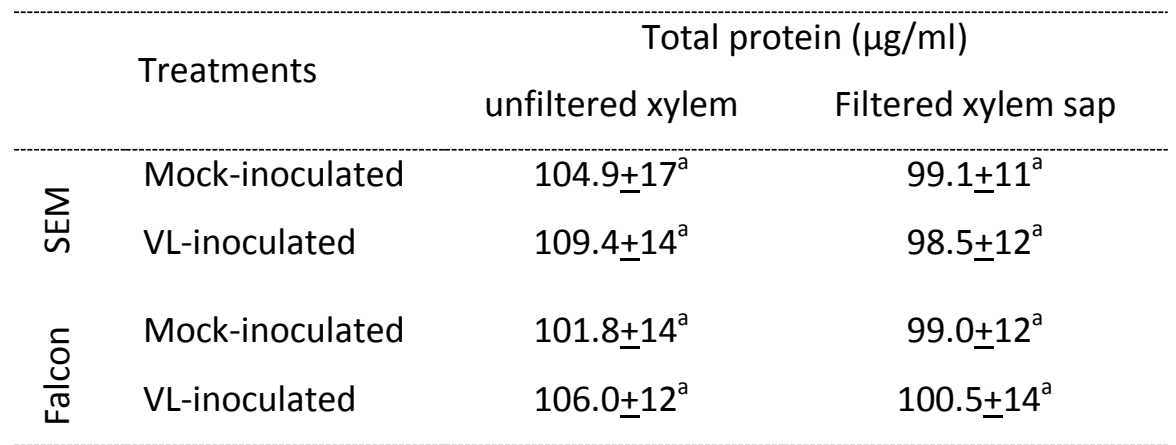

Protein content was determined by with the Bradford method using bovine serum albumin (BSA) as a standard. Means \pm standard deviation were obtained from three biological replicates. Mean values assigned with the same superscript are not significantly different at $\mathrm{P}=0.05$. 


\subsubsection{Analysis of $V$. longisporum growth in xylem sap extracted from plants at different age}

In this independent study, in addition to the cultivar-related VL resistance, the growth of VL in xylem sap extracted from different aged plants was investigated. Similar to the previous experiment (section 3.4.2), this study was accomplished in two settings, greenhouse experiments and in vitro bioassays. Further to analyse the relationship between xylem sap constituents and in vitro fungal growth, the sugar concentration in xylem sap samples collected from different aged plants was quantified. Two B. napus genotypes expressing contrasting levels of resistance to VL, namely Aviso (resistant) and Falcon (susceptible) were used. Xylem sap was extracted from mock and VL-infected plants in 2-3 day intervals during the period between 13 to $28 \mathrm{DPI}$. For the bioassay, xylem saps extracted from different aged plants were inoculated with spores of VL and fungal growth was daily measured with a spectrophotometer as above.

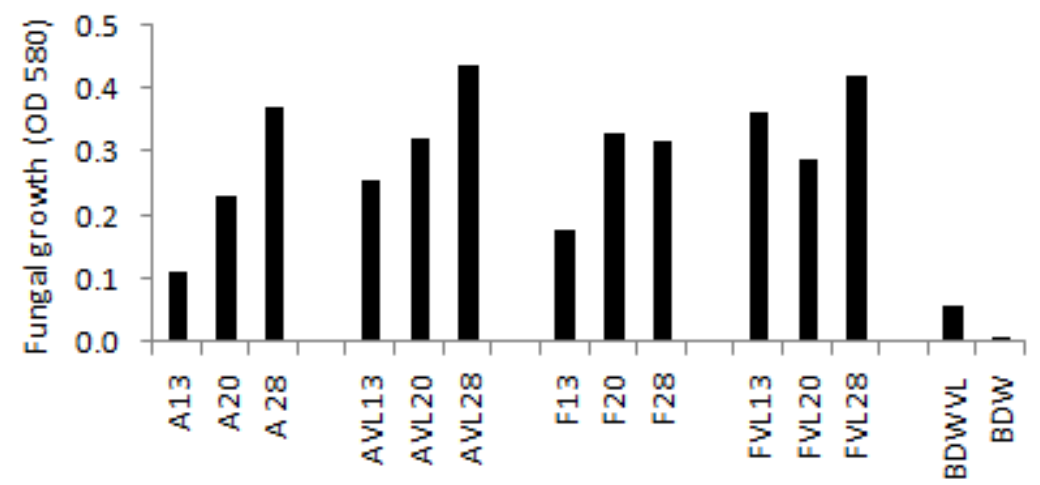

Figure 3.6 Spectrophotometric measurement of Verticillium longisporum growth five days after incubation on xylem sap extracted from resistant (Aviso) and susceptible (Falcon) Brassica napus plants. Treatments: A, Aviso mock-inoculated. AVL, Aviso VL-inoculated. F, Falcon mock-inoculated. FVL, Falcon VL-inoculated. Numbers after treatments refer to DPI at which xylem sap was extracted. BDWVL, Fungal growth on sterile bi-distilled water. BDW, sterile bi-distilled water without VL inoculum.

Table 3.4 Average growth rate of $V$. longisporum in xylem sap extracted from different aged mock and Verticillium longisporum inoculated resistant and susceptible Brassica napus genotypes.

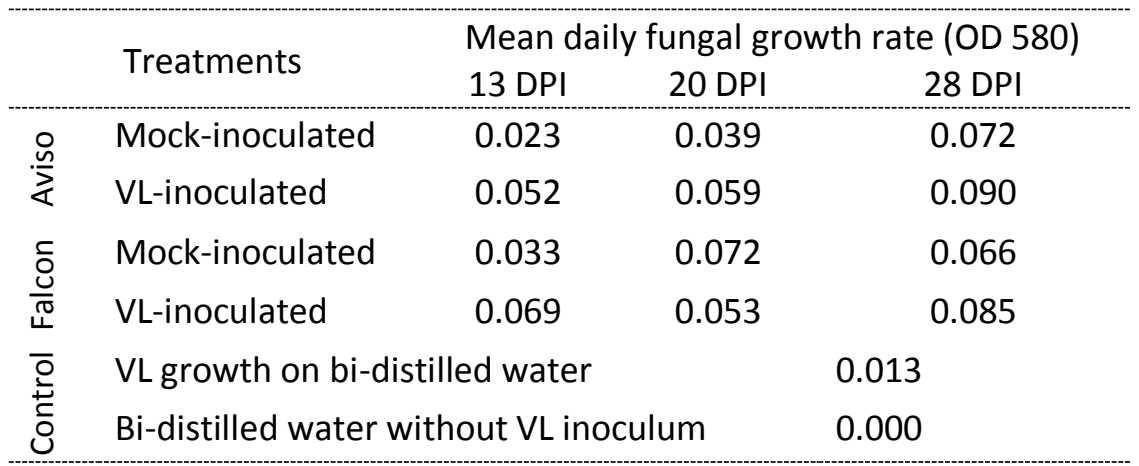

Daily fungal growth rate was calculated by subtracting absorbance value of previous day measurements of each treatment. Data shown is average of four time points (2, 3, 4 and $5 \mathrm{DPI})$. DPI, days post incubation. 


\subsubsection{Xylem sap bioassay}

Results obtained from the in vitro bioassay were consistent and strongly comparable with the independently conducted first experimental series with two different genotypes (section 3.4.2). Xylem sap derived from mock and VL-infected plants of both resistant and susceptible genotypes was equally suitable for the growth of VL (Fig. 3.7). Unlike the control medium (sterile bi-distilled water), a significantly higher fungal growth was measured after two days of incubation. However, the growth of VL in xylem sap from resistant and susceptible plants was not significantly different. Likewise, xylem sap from VL-infected resistant and susceptible same age plants did not cause significant change on the in vitro growth of VL (Fig. 3.6). Analysis of variance of fungal growth in xylem sap extracted from earlier time points $(13,18,20,22$, and $26 \mathrm{DPI})$ also showed comparable growth of VL in all xylem sap samples (data not shown).

Interestingly, comparison of fungal growth in xylem sap from plants at different age showed enhanced fungal growth of VL in xylem sap from older plants. Analysis of the daily fungal growth rate in xylem sap samples extracted in a weekly interval also showed a significantly positive correlation between in vitro fungal growth rate and plant age (Table 3.6). A slightly increased fungal growth rate was observed in xylem sap samples of VL-inoculated Aviso plants. Regarding the pattern of fungal growth in xylem sap derived from the susceptible genotype Falcon, a similar increase in growth rate was recorded from older plant xylem sap samples. It is noteworthy to mention, that the average daily fungal growth rate recorded in xylem sap at $13 \mathrm{DPI}$ of mock and VL-inoculated plants was OD 0.033 and 0.069 , respectively. The respective growth rate values at 28DPI were OD 0.066 and 0.085 . Growth rates at $20 \mathrm{DPI}$ seemed to be inconsistent. As with genotype Aviso, an increased rate of fungal growth at 13 and $28 \mathrm{DPI}$ was also noticed in xylem sap samples collected from VL infected Falcon plants.

\subsubsection{Xylem sap total carbohydrate content}

In order to investigate the relationship between fungal growth and xylem sap sugar content, the total carbohydrate $(\mathrm{CHO})$ concentration in xylem sap samples used in the bioassays was quantified using a glucose standard (Appendix 3.2). Results obtained from different aged plants showed comparable $\mathrm{CHO}$ concentrations in xylem sap of susceptible and resistant genotypes. Even though statistically insignificant, plant age dependent effect of VL infection was noticed. At 14, 18 and 21 DPI, CHO levels in xylem sap of VL-infected plants were slightly increased. In contrast, CHO levels in xylem sap at 28 DPI of VL-infected plants were relatively lower. Interestingly, it was found that xylem sap sugar concentration was significantly dependent on plant age. Compared to earlier time points, total xylem sap $\mathrm{CHO}$ 
concentration in $28 \mathrm{DPI}$ old plants of both mock or VL-infected resistance and susceptible genotypes was significantly higher than in younger plants (Table 3.5).
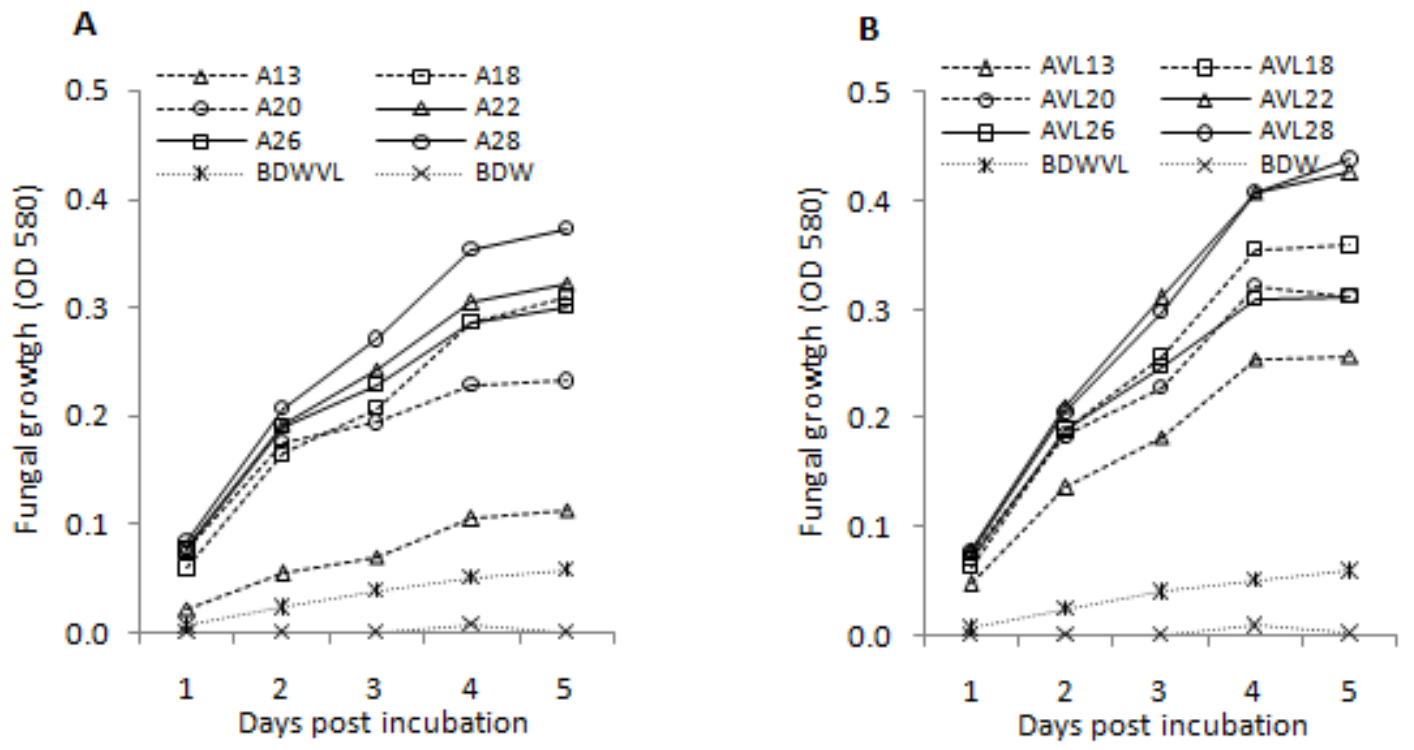

\section{C}
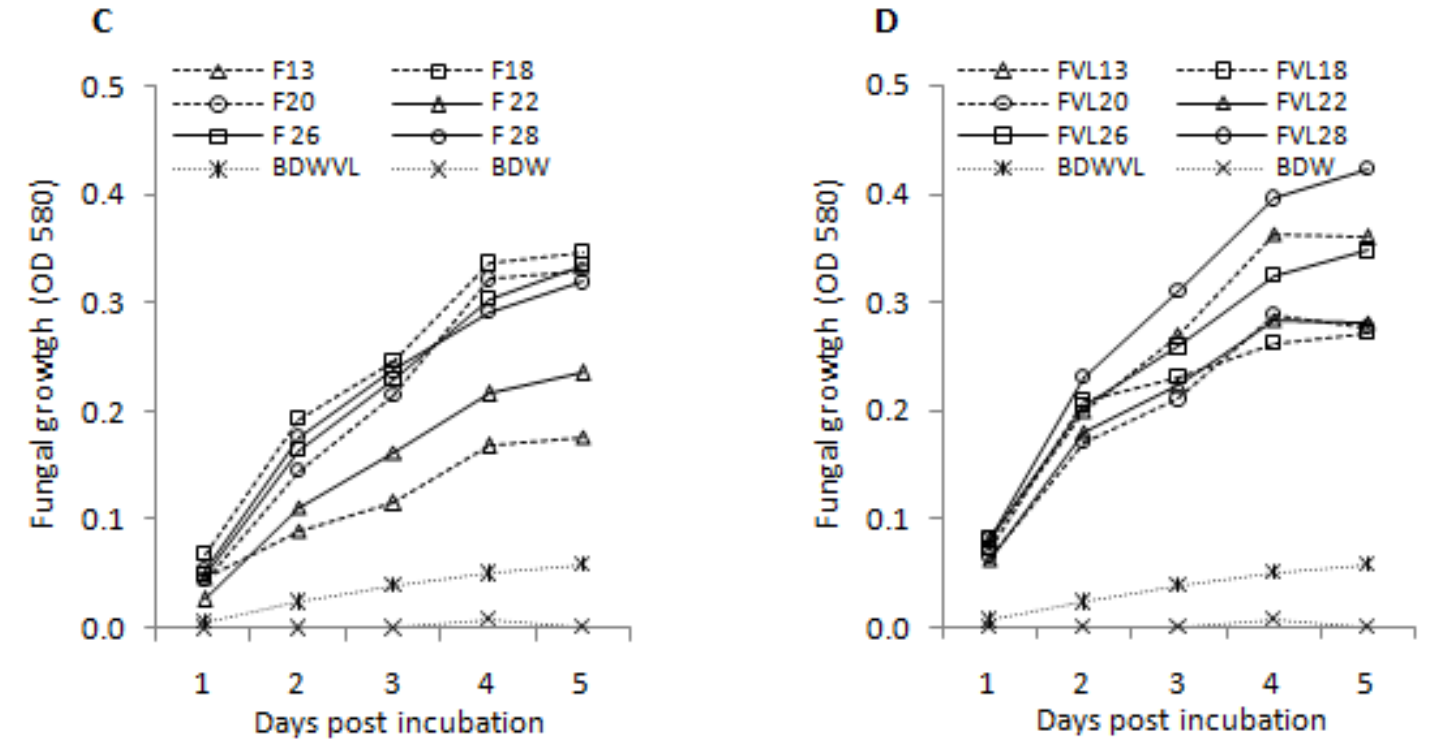

Figure 3.7 Spectrophotometric measurement of Verticillium longisporum growth in xylem sap extracted from resistant (Aviso) and susceptible (Falcon) Brassica napus genotypes. A, fungal growth in xylem sap extracted from Aviso, mock-inoculated. B, fungal growth in xylem sap extracted from Aviso, VL-inoculated. C, fungal growth in xylem sap extracted from Falcon, mock-inoculated. D, fungal growth in xylem sap extracted from Falcon, VLinoculated. Treatments: A, Aviso mock-inoculated. AVL, Aviso VL-inoculated. F; Falcon mock-inoculated. FVL, Falcon VL-inoculated. Numbers after treatments refer to DPI at which xylem sap was extracted. BDWVL, Fungal growth on sterile bi-distilled water. BDW, sterile bi-distilled water without VL inoculum.

\subsubsection{Correlation analysis}

With correlation analysis, the relationship between in vitro fungal growth and xylem sap sugar and protein concentrations was studied. Furthermore, the correlation between plant age and fungal growth rate in xylem sap and the correlation among disease parameters were analysed. Results showed a signif- 
Table 3.5 Concentrations of xylem sap total carbohydrate in Brassica napus genotypes resistant (Aviso) and susceptible (Falcon) to Verticillium longisporum.

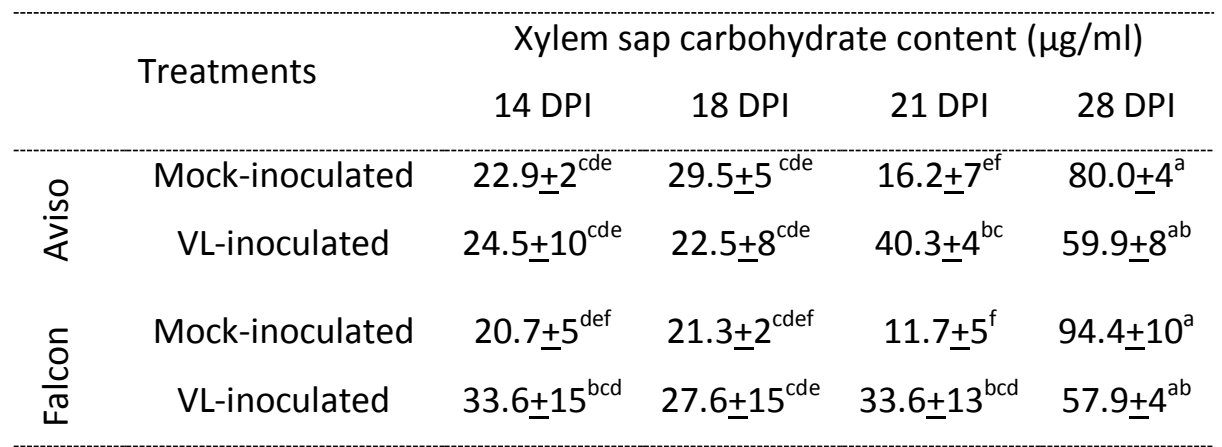

Carbohydrate content was determined using the phenol-sulphuric acid method. Known concentrations of glucose were used as standards. Means \pm standard deviation were obtained from three biological replicates. Mean values assigned with the same superscript are not significantly different at $P=0.05$.
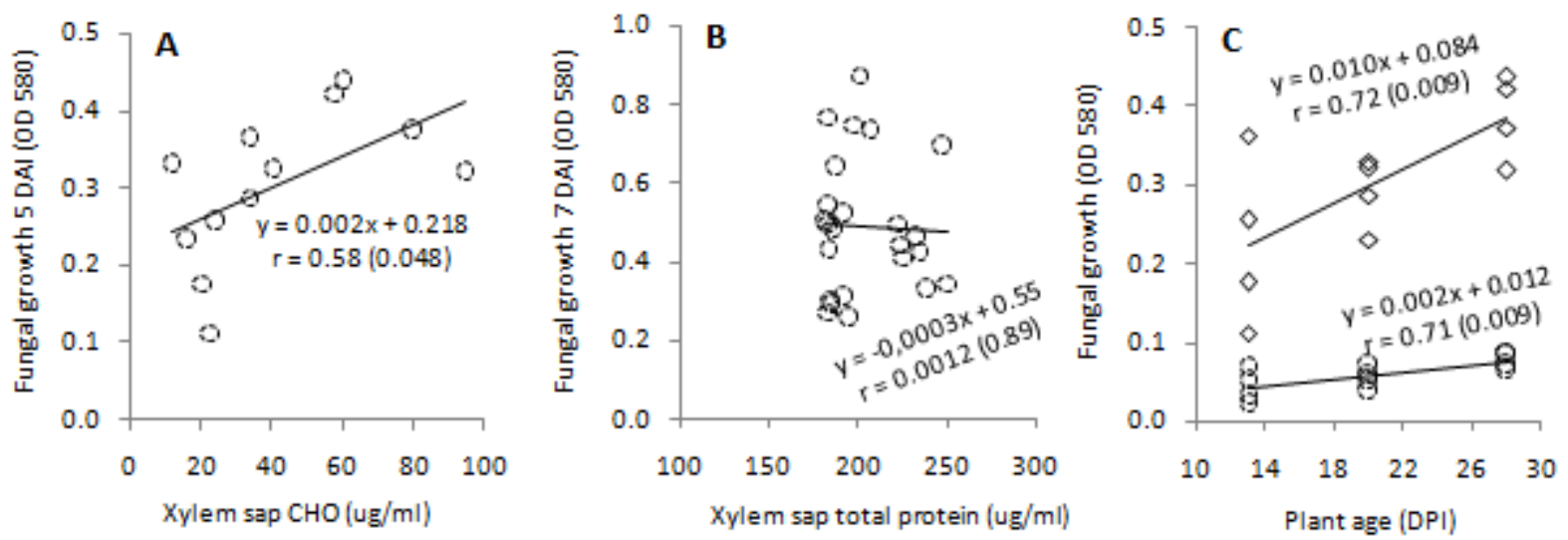

Figure 3.8 Relationships between Verticillium longisporum growth and constituents and age of xylem sap extracted from mock and VL-inoculated resistant and susceptible Brassica napus plants. P-values are indicated in brackets. Pvalues less than 0.05 indicate significant correlations.

A. Pearson's correlation between total xylem sap protein and fungal growth five days after incubation. Spores of VL were grown in xylem sap extracted 13, 20 and 28 DPI. Total carbohydrate concentration in xylem sap extracted at 14, 21 and 28 DPI was quantified using known concentrations of glucose standard solutions.

B. Pearson's correlation between total xylem sap proteins and fungal growth seven days after incubation. Spores of VL were grown on xylem sap extracted at $28 \mathrm{DPI}$. Total protein concentration in xylem sap extracted at 28 DPI was quantified using known concentrations of BSA standard solutions. DPI, Days post inoculation with Verticillium longisporum. DAl, days after incubation in xylem sap. CHO, carbohydrate.

C. Pearson's correlation between plant age at the time of xylem extraction and fungal growth five days after incubation (diamond) or daily fungal growth rate (circles). Data from weekly interval samples $(13,20$ and 28 DPI) were used.

-cant and strong positive correlation between xylem sap sugar concentration and growth of VL in xylem sap (Fig. 3.8A). A similarly strong correlation was identified between fungal growth and plant age at the time of xylem sap extraction (Fig. 3.8C). In contrast, no correlation was found between rate of fungal growth and concentrations of total proteins in xylem sap (Fig. 3.8B). Results of Pearson's correlation 
analysis of phenotypic and molecular disease parameters from greenhouse experiments also showed strong relationships. The highest correlation was found between AUDPC and plant height $(r=-0.92)$ followed by AUDPC and VL DNA ( $r=0.89$ ). Very strong negative correlations (ranging between -0.73 and 0.79) existed among the rest of disease parameters except for plant height and stem diameter ( $r=0.49)$ where the correlation was not significant. The relationship between disease and SDM yield was significantly strong, verifying the consistent responses of the genotypes to VL infection (Table 3.6).

Table 3.6 Correlation coefficients describing relationships among disease and agronomic parameters measured in the greenhouse experiments at $28 \mathrm{DPI}$.

\begin{tabular}{lcccc} 
& Net AUDPC & Plant height & Hypocotyl diameter & Shoot DM \\
\hline VL DNA & $0.89^{* * *}$ & $-0.79^{* * *}$ & $-0.73^{* *}$ & $-0.69^{*}$ \\
Net AUDPC & & $-0.92^{* * *}$ & $-0.77^{* * *}$ & $-0.81^{* *}$ \\
Plant height & & $0.49^{\text {ns }}$ & $0.78^{* *}$ \\
Hypocotyl diameter & & & $0.61^{*}$
\end{tabular}

Analysis was performed using Pearson (product-moment) correlation (STATISTICA version 10 StatSoft, inc. 2011). Minus signs indicate negative correlations. $\mathrm{ns}$, not significant. ${ }^{*}$, significant at $\mathrm{P}=0.05 .{ }^{* *}$, significant at $\mathrm{P}=0.01 .{ }^{* * *}$, significant at $\mathrm{P}=0.001$. DPI, Days post inoculation with Verticillium longisporum. Net AUDPC, net area under disease progress curve, VL DNA, Verticillium longisporum DNA in hypocotyl. DM, dry matter.

\subsection{Discussion}

The focus of this study was identification of xylem sap residing cultivar-related $V$. longisporum resistance factors in oilseed rape. The study utilized three B. napus genotypes with differential degree of resistance against VL. The overall study was accomplished by different experimental settings involving greenhouse experiments, in vitro bioassays and biochemical analyses. Initially, the greenhouse experiments were conducted with the aim of collecting xylem sap and verifying the responses of the three B. napus genotypes to VL infection. Analysis of net AUDPC, stunting effects and stem thickness together with quantification of VL DNA showed significantly lower levels of disease in genotypes SEM and Aviso, confirming resistance of these genotypes to VL. In contrast, infected Falcon plants showed a fast development of disease symptoms and a significantly higher level of disease severity (see section 3.4.1). The strong positive correlation between AUDPC values and qPCR data as well as the negative correlation of AUDPC and fungal biomass with plant height, stem thickness and shoot biomass measurements (Table 3.6) also consistently verified resistance and susceptible responses of the genotypes used in this study. In general, molecular and phenotypic greenhouse disease evaluation results confirmed the observations of previous field and greenhouse resistance evaluations that showed VL-resistance of 
genotype Aviso (Keunecke, 2009) and SEM (Keunecke, 2009; Eynck et al., 2009b) and susceptibility of cultivar Falcon (Knüfer, 2013; Eynck et al., 2009b; Rygulla et al, 2007b).

In the first experiment, the antifungal activity of xylem sap samples extracted from 28 DPI old plants was investigated. This time point was chosen based on previous studies that identified this stage as critical period for significant accumulation of VL-resistance factors in resistant B. napus plants (Eynck et al., 2009b; Obermeier et al., 2013). Results showed that B. napus xylem sap provides a suitable medium for the growth of VL. Spore germination and further fungal growth were detected 2-3 days after incubation. From 5 DPI onwards, proliferated fungal growth that covered the whole area of the microtitre plate wells were observed. Comparison of fungal growth in xylem sap of resistant and susceptible plants however showed no significant difference until 7 DPI (Fig. 3.4 and 3.7; Appendix 3.3). Further comparisons of fungal growth in xylem sap of both genotypes revealed the absence of infection induced VL-resistance factor contributing to a significant reduction of in vitro fungal growth. Singh et al., (2012) however suggested a possible role of pre-existing xylem sap proteins in defence of OSR against VL. In contrast, we observed slightly better fungal growth in xylem sap derived from infected plants, compared to control plants. Enhanced growth of the $V$. dahliae in vascular fluid of nematode infected cotton cultivars have been reported previously (Katsantonis et al., 2005). Despite the slightly reduced fungal growth on filtered xylem sap treatments, no significant effect of xylem sap filtration on the rate and level of VL growth was noticed. This observation is partly explained by the similar concentrations of total soluble protein measured in xylem fluid of the resistant and susceptible genotypes (Table 2) which is comparable concentration to previous reports in B. napus (Buhtz et al., 2004), cotton (Singh et al., 2013) and olive seedlings (Neumann et al., 2010). On the other hand, the relatively higher protein concentrations found in infected plants indicate a slight, cultivar independent and infection-induced increase of protein levels in B. napus xylem sap. Since fungi use proteins as a source of nitrogen and sulphur (Walker and White, 2011), it is not surprising that enhanced fungal growth was observed in xylem sap samples with high protein concentrations. It was also observed that total soluble protein concentrations were slightly reduced in filtered xylem samples. This might be due to a slight protein binding property of the syringe filter (Whatman Anotop 10 plus sterile) used for the purification of xylem sap samples. Still, reduction of protein content in filtered xylem sap samples was not significantly different from the non-infected samples. This again is in agreement with the fungal growth bioassay results where insignificant difference in fungal growth between filtered and unfiltered xylem sap samples was observed. 
The bioassay results from the present study are in agreement with some of the observations reported by Floerl el al. (2008) who compared leaf apoplast and xylem sap compositions of non-infected and VLinfected susceptible B. napus plants. According to this study, B. napus xylem sap provides a suitable medium for growth of VL and VL infection did not affect concentrations of xylem sap total proteins. In contrast, the study also showed a significant reduction of VL growth on xylem sap derived from infected VL-susceptible B. napus plants. As possible factors of VL growth inhibition, enhanced accumulation of two xylem sap proteins (PR-4 and $\beta$-1,3-glucanase) were suggested (Floerl el al., 2008). More than 69 pre-existing secreted enzymes and proteins with various potential functions related to plant defence, cell wall modification and strengthening are known to exist in xylem sap of B. napus (Kehr et al., 2005). However, whether the concentrations or the occurrence of these proteins is variable among $B$. napus varieties is not known. In addition, it is not known whether pathogenic or symbiotic interactions cause changes in B. napus xylem sap composition. In tomato for example, infection of resistant and susceptible plants with virulent and avirulent strains $V$. albo-atrum caused a general increase in the xylem sap amino acid concentration (Dixon and Pegg, 1972). In contrast, infection with the symbiotic bacterium Bradyrhizobium japonica, unlike the elicitor of the pathogenic fungi Phytophthora sojae, caused no significant increase in concentrations of xylem sap proteins in soybean (Subramanian et al, 2009).

With regard to defence related plant hormones, Ratzinger et al. (2009) reported the pre-existence of salicylic acid (SA), jasmonic acid (JA) and abscisic acid (ABA) in xylem sap of VL-susceptible rapid cycling rape (B. napus). This study clearly showed that following VL infection, concentration of SA is significantly increased while levels of JA and ABA are unaffected. Other studies also showed a lack of correlation between SA levels and VL-resistance in B. napus. Kamble et al. (2013) reported VL induced increased levels of SA in hypocotyl and leaf extracts of a susceptible winter OSR cultivar. This study also showed no role of the SA pathway marker gene (PR-1) in VL-resistance. Another study that compared susceptible and resistant genotypes revealed that VL infection induced significantly higher levels of SA in stem extracts of VL-susceptible cultivar compared to a resistant genotype (Siebold, 2012).

The most striking observation of the present study was the differential growth of VL in xylem saps from different aged plants. Unlike the sap of younger (13 to $21 \mathrm{DPI}$ old) plants, xylem sap from 28 DPI old resistant and susceptible plants provided the highest level of fungal growth (Fig. 3.8; Table 3.4). This finding may explain disease development under field conditions. During early growth stages of $B$. napus in the field, VL has a characteristic long latency period. Once the crop reaches maturity stages, however, first symptoms as one-sided brownish discoloration of the stem are developed (Knüfer, 2013). Subsequently, the fungus grows rapidly and produces microsclerotia, which later burst out of the stem 
tissue (Leino, 2006). VL might sense signals of crop maturity stages that might be present in xylem tissue. With these signals, the fungus might detect that the plant is on the way to complete its life cycle and this situation might induce an increase in growth of VL resulting in bursting out of the vascular tissues in the shoot and produce resting structures.

Given the fact that large amounts of sugar is present and transported via xylem (Loescher et al., 1990; Iwai et al., 2003) and increased sugar concentrations were found in xylem sap of older plants (Table 3.5), it is not surprising that we observed enhanced growth of VL in xylem sap of older plants. Increased concentration of sugar is suggested as an indigenous signal for transition from juvenile to adult stages of different plant species (Yu et al., 2013). In tomato, xylem sap sugar concentration in healthy and nematode infected plants sharply increased with increase in plant age (Wang and Bergeson, 1974). In Arabidopsis, compared to 15 day old plants, significantly higher levels of sugars (Glc, Suc and Fruc) were detected in shoot extracts of 60 days old plants (Yu et al., 2013). In the present study, the presence of about equal amounts of $\mathrm{CHO}$ irrespective of plant genotype also explains the equal growth of $\mathrm{VL}$ in xylem sap of resistant and susceptible plants. Similar results showing no correlation between xylem sap sugar content and resistance to the vascular dutch elm disease in different Ulmaceae species have been reported long time ago (Singh and Smalley, 1969).

Overall, our results support previous studies that showed that physical or cell wall bound resistance factors play a key role in cultivar-related resistance of B. napus against VL. Eynck et al. (2009b) first identified the hypocotyl tissue as a major battlefield of the B. napus-VL interaction and demonstrated the significance of physical defence structures. These mechanisms include de novo formation and a significant build-up of lignin as well as reinforcement of tracheary elements with cell wall bound phenolics and lignin. In another recent study focusing on the identification QTLs involved in VL resistance in different $B$. napus genetic backgrounds, a strong correlation between phenylpropanoids and VL resistance was found. More importantly, this study showed lower concentrations of the major lignin precursor (caffeic acid) in resistant lines compared to susceptible plants, suggesting substantial incorporation of this compound into lignin of cell walls (Obermeier et al., 2013), thus again indicating the substantial role of physical barriers in cultivar-related VL resistance in B. napus. In general, the findings of the present study suggest that soluble xylem sap constituents are not playing a role as major resistance factors for cultivar-related winter OSR resistance against $V$. longisporum. 


\subsection{References}

Atwell B. J., Kriedemann P. E., Turnbull C.G.N. 1999. Plants in action: adaptation in nature, performance in cultivation. Macmillan Education. South Yarra, Melbourne, Australia.

Bradford M. M. 1976. A rapid and sensitive method for the quantitation of microgram quantities of protein utilizing the principle of protein-dye binding. Analytical Biochemistry, 72: 248-254.

Broekaert W. F., Terras F. R. G., Cammue B. P. A., Vanderleyden J. 1990. An automated quantitative assay for fungal growth inhibition. FEMS Microbiology Letters, 69: 55-60.

Buhtz A., Kolasa A., Arlt K., Walz C. and Kehr J. 2004. Xylem sap protein composition is conserved among different plant species. Planta, 219: 610618.

Carré P. and Pouzet A. 2014. Rapeseed market, worldwide and in Europe. Oilseeds and fats crops lipids, 21 (1) D102.

Dixon G. R. and Pegg G. F. 1972. Changes in amino-acid content of tomato xylem sap following infection with strains of Verticillium albo-atrum. Annals of Botany, 36:147-54.

Dunker S., Keunecke H., Steinbach P., von Tiedemann A. 2008. Impact of Verticillium longisporum on yield and morphology of winter oilseed rape (Brassica napus) in relation to systemic spread in the plant. Journal of Phytopathology, 156: 698-707.

Eynck C., Koopmann B. and von Tiedemann A. 2009a. Identification of Brassica accessions with enhanced resistance to Verticillium longisporum under controlled and field conditions. Journal of Plant Diseases and Protection, 116(2), 63-72.

Eynck C., Koopmann B., Karlovsky P., and von Tiedemann A. 2009b. Internal resistance in winter oilseed rape inhibits systemic spread of the vascular pathogen Verticillium longisporum. Phytopathology, 99:802-811.

Floerl S., Druebert C., Majcherczyk A., Karlovsky P., Kües U. and Polle A. 2008. Defence reactions in the apoplastic proteome of oilseed rape (Brassica napus var. napus) attenuate Verticillium longisporum growth but not disease symptoms. BMC Plant Biology, 8:129.

Gomez K. A. and Gomez A. A. 1984. Statistical procedures for agricultural research, $2^{\text {nd }}$ ed., John Wiley and Sons, New York.

Happstadius I., Ljungberg A., Kristiansson B. and Dixelius C. 2003. Identification of Brassica oleracea germplasm with improved resistance to Verticillium wilt. Plant Breeding, 122:30-34.

Heale J. B., and Karapapa K. V. (1999) The Verticillium threat to Canada's major oilseed crop: Canola. Canadian Journal of Plant Pathology, 21(1):1-7.

Iwai H., Usui M., Hoshino H., Kamada H., Matsunaga T., Kakegawa K., Ishii T. and Satoh S. 2003. Analysis of sugars in squash xylem xap. Plant and Cell Physiology, 44(6): 582-587. 
Inderbitzin P., Davis R. M., Bostock R. M. and Subbarao K. V. 2011a. The Ascomycete Verticillium longisporum is a hybrid and a plant pathogen with an expanded host range. PLOS ONE, 6(3): e18260.

Johansson A., Goud J. C. and Dixelius C. 2006 Plant host range of Verticillium longisporum and microsclerotia density in Swedish soils. European Journal of Plant Pathology, 114:139-149.

Kamble A., Koopmann B. and von Tiedemann A. 2013. Induced resistance to Verticillium longisporum in Brassica napus by b-aminobutyric acid. Plant Pathology,62:552-561.

Karapapa V. K., Bainbridge B. W. and Heale J. B. 1997. Morphological and molecular characterization of Verticillium longisporum comb. nov., pathogenic to oilseed rape. Mycological Research, 101:1281-1294.

Kehr J., Buhtz A. and Giavalisco P. 2005. Analysis of xylem sap proteins from Brassica napus. BMC Plant Biology, 5(11):1-13.

Keunecke H.. 2009. Einfluss von Kohlfliegenbefall auf die Infektion und Schadwirkung von Verticillium longisporum und Phoma lingam an Raps. PhD thesis. Georg-August-Universität Göttingen, Göttingen, Germany.

Knüfer J. 2013. Improvement of winter oilseed rape resistance to Verticillium longisporum - Assessment of field resistance and characterization of ultrastructural plant responses. PhD thesis. GeorgAugust-Universität Göttingen, Göttingen, Germany.

Leino M. 2006. Fungal diseases on oilseed rape and turnip rape. Norrköping, Sweden.

Loescher W. McCamant H., T., and Keller J. D. 1990. Carbohydrate reserves, translocation, and storage in woody plant roots. HortScience, 25(3):274-281.

Masuko T., Minami A., Iwasaki N., Majima T., Nishimura S. and Lee Y. C. 2005. Carbohydrate analysis by a phenol-sulfuric acid method in microplate format. Analytical Biochemistry, 339:69-72.

Neumann P. M., Weissman R., Stefano G. and Mancuso S. 2010. Accumulation of xylem transported protein at pit membranes and associated reductions in hydraulic conductance. Journal of Experimental Botany, 61(6):1711-1717.

Obermeier C., Hossain M. A., Snowdon R., Knüfer J., von Tiedemann A. and Friedt W. 2013. Genetic analysis of phenylpropanoid metabolites associated with resistance against Verticillium longisporum in Brassica napus. Molecular Breeding, 31:347-361.

Ratzinger A., Riediger N., von Tiedemann A. and P. Karlovsky. 2009. Salicylic acid and salicylic acid glucoside in xylem sap of Brassica napus infected with Verticillium longisporum. Journal of Plant Research, 122:571-579. 
Rygulla W., Friedt W., Seyis F., Lühs W., Eynck C., von Tiedemann A. and R. J. Snowdon. 2007a. Combination of resistance to Verticillium longisporum from zero erucic acid Brassica oleracea and oilseed Brassica rapa genotypes in resynthesized rapeseed (Brassica napus) lines. Plant Breeding, 126:596-602.

Rygulla W., Snowdon R. J., Eynck C., Koopmann B., von Tiedemann A., Lühs W. and Friedt W. 2007b. Broadening the genetic basis of Verticillium longisporum resistance in Brassica napus by interspecific hybridization. Phytopathology, 97:1391-1396.

Rygulla W., Snowdon R. J., Friedt W., Happstadius I., Cheung W. Y. and Chen D. 2008.Identification of quantitative trait loci for resistance against Verticillium longisporum in oilseed rape (Brassica napus). Phytopathology, 98(2):215-221.

Scholander P. F., Hammel H. T., Bradstreet E. D., and Hemmingsen E. A. 1965. Sap pressure in vascular plants: Negative hydrostatic pressure can be measured in plants. Science, 16,148(3668):339-46.

Siebold M. 2012. Effects of rising air and soil temperatures on the life cycle of important pathogens in oilseed rape (Brassica napus L.) in Lower Saxony. PhD thesis. Georg-August-Universität Göttingen, Göttingen, Germany.

Singh D. and Smalley E. B. 1969. Nitrogenous and carbohydrate compounds in the xylem sap of Ulmaceae species varying in resistance to Dutch elm disease. Canadian Journal of Botany, 47(2):335-339.

Singh H., Dixit S., Verma P. C., and Singh P. K. 2013. Differential peroxidase activities in three different crops upon insect feeding. Plant Signaling \& Behavior, 8(10):e25615.

Singh S., Braus-Stromeyer S. A., Timpner C., Valerius O., von Tiedemann A., Karlovsky P., Druebert C., Polle A., and Braus G. H. 2012. The plant host Brassica napus induces in the pathogen Verticillium longisporum the expression of functional catalase peroxidase which is required for the late phase of disease. Molecular Plant-Microbe Interactions, 25(4):569-581.

Subramanian S., Cho U. H., Keyes C. and Yu O. 2009. Distinct changes in soybean xylem sap proteome in response to pathogenic and symbiotic microbe interactions. BMC Plant Biology, 9(119):1-11.

Veronese P., Narasimhan M. L., Stevenson R. A., Zhu J. K., Weller S. C., Subbarao K. V. and Bressan R. A. 2003. Identification of a locus controlling Verticillium disease symptom response in Arabidopsis thaliana. The Plant Journal, 35(5):574-587.

Wang E. L. H. and Bergeson G. B. Biochemical changes in root exudate and xylem sap of tomato plants infected with Meloidogyne incognita. Journal of Nematology, 6(4): 194-202.

Yadeta K. A. and Thomma B. P. H. J. 2013. The xylem as battleground for plant hosts and vascular wilt pathogens. Frontiers in Plant Science, 4(97):1-12. 
Yu S., Cao L., Zhou C. M., Zhang T. Q., Lian H., Sun Y., Wu J., Huang J., Wang G. and Wang J. W. 2013. Sugar is an endogenous cue for juvenile-to-adult phase transition in plants. elife, 2:e00269.

Zeise K. and von Tiedemann A. 2002. Host specialization among vegetative compatibility groups of Verticillium dahliae in relation to Verticillium longisporum. Journal of Phytopathology, 150, 112119. 


\section{Physiology, gene expression and agronomic performance of winter oilseed rape under drought stress and infection with Verticillium longisporum*}

*Parts of this chapter have been submitted for publication to Phytopathology. Manuscript ID PHYTO-0716-028/0-R: Lopisso T. D., Knüfer J., Koopmann B. and von Tiedemann A. The vascular pathogen Verticillium longisporum does not affect water relations and plant responses to drought stress of its host, Brassica napus.

Department of Crop Sciences, Division of Plant Pathology and Crop Protection, Georg August University, Grisebachstr. 6, 37077 Göttingen, Germany; 2Current address: Strube Research GmbH \& Co. KG, 38387 Söllingen, Germany

\subsection{Introduction}

Oilseed rape (OSR) ranks among the top four largely produced oil crops in the world. The economic importance of OSR is increasing overtime and at present it is intensively cultivated in more than 60 countries worldwide (FAOSTAT, 2014). However, the increasing intensive production particularly due to the growing demands for biodiesel (Carré and Pouzet, 2014; Zentková and Cvengrošová, 2013) favored the emergence of new economically important diseases such as Verticillium longisporum. $V$. longisporum recently evolved as a host specific vascular pathogen with host specificity to cruciferous species (Karapapa et al., 1997; Inderbitzin et al., 2011a) and a potential to cause substantial yield losses (Dunker et al., 2008). Since the 1990s, it is becoming a major threat to oilseed rape production particularly in northern European countries (Dunker et al, 2008). Simultaneously, the geographical distribution of $V$. longisporum is widening and more recently its presence is reported from Japan, Illinois and the UK (Babadoost et al., 2004; Ikeda et al., 2012; Gladders et al., 2011; CABI/EPPO, 2011).

As a soil-borne pathogen, VL infects OSR by direct penetration of lateral roots and root hairs. Once it is inside the root cortex cells, it starts to systemically colonize root and shoot xylem vessels where it spends most of its life cycle (Zhou et al., 2006; Eynck et al., 2007). The typical symptoms caused by VL begin with the development of one-sided yellowing of leaves followed by vein clearing, complete foliar chlorosis and premature senescence and ripening. Artificially inoculated susceptible plants under controlled conditions also show severe stunting of shoots, reduction of root length and excessive production of side branches. Towards crop maturity, infected plants show brownish stripes along the stems and subsequently, the pathogen bursts out of the xylem vessels and produces blackish layers of elongate microsclerotia underneath the stem epidermis, in the stem pith and roots. When diseased plants start senescence and during crop harvest, enormous amounts of highly durable microsclerotia are 
released in the soil where they may survive in dormancy for more than a decade (Heale and Karapapa, 1999; Berg et al., 1999; Leino, 2006; Eynck, 2008).

The nature of systemic colonization and the extremely durable survival of VL greatly have hampered its control and limited the options available for combating this disease. To date, there are no fungicides that effectively work against VL and cultural methods such as crop rotation are not reliable. Consequently, current control of VL solely relies on the use of OSR genotypes with enhanced resistance (Dunker et al., 2008). Previous breeding efforts aimed at developing resistant varieties appeared to be successful. At present, OSR genotypes that show different levels of resistance towards VL are available (Eynck et al., 2009a; Rygulla et al., 2007b; Rygulla et al., 2007a). It is also known that pathogen-induced histological and biochemical changes in the vascular tissue of infected OSR, including accumulation of soluble and cell wall-bound phenolics, vascular occlusions and cell wall-bound lignin, are major resistance mechanisms responsible for restricting VL below the hypocotyl and preventing its further spread into the shoot (Eynck et al., 2009b). Obermeier et al. (2013) who recently conducted a QTL analysis of phenylpropanoid metabolites associated with VL resistance provided additional evidence for the involvement of phenolic compounds and lignin in B. napus resistance against VL. Nevertheless, whether these defence mechanisms and structural changes in the xylem vessels are at the expense of yield or have further negative secondary consequences on water and nutrient relations, particularly during drought seasons, is not known.

In an agronomic context, drought refers to a situation where the amount of moisture in the soil no longer meets the needs of a particular crop (Stanke et al., 2013). This is one of the most adverse abiotic factors threatening crop growth and productivity (Anjum et al., 2011). Drought was long considered a phenomenon affecting mainly developing countries in the arid regions. But, this may change since these days some areas in the temperature climate regions are also experiencing drought. For instance, due to an increase in frequency, duration or intensity of low flows, the drought situation in some European regions has become more severe in the last decades. Besides, a further increase of drought driven by climate change particularly in southern European areas is expected (Lehner and Döll, 2001). According to long-term climate change projections, there could be more frequent and severe droughts in certain areas of the world and such impact could also become more marked in climate zones where oilseed rape is grown.

Drought generally affects plant growth and development by interfering with water and nutrient relations, photosynthesis, assimilate partitioning, and other related metabolic processes. Disturbance of 
the aforementioned processes in turn leads to reduced growth, wilting, leaf senescence and finally severe yield losses and complete death of plants (Farooq et al., 2009). In Brassica crops, drought stress severely affects leaf chlorophyll content, photosynthesis, transpiration and stomatal conductance (Din et al., 2011; Singh et al., 2009). It causes a delayed and prolonged period of flowering and reduces leaf area index, branching, silique production, plant biomass formation, and grain yield (Sadaqat et al., 2003; Müller et al., 2010; Zakirullah et al., 2000). Drought also reduces seed oil content (Sinaki et al., 2007) and increases the glucosinolate content which in turn influences oil quality (Moghadam et al., 2011; Jensen et al., 1996) and susceptibility to diseases and insect herbivory (Mithen, 1992).

As a response to drought stress, plants display a range of physiological, morphological, biochemical and molecular changes. Mechanisms of drought resistance in plants vary among species, varieties and even depend on plant age. Nevertheless, all mechanisms involve either avoidance or tolerance of dehydration and this can be achieved either by enhancement of water use efficiency or reduction of transpiration losses (Blum, 2005). The mechanisms of drought tolerance in plants include possession of prolific and deep root systems, smaller and succulent leaves, reduced numbers of stomata, and production of trichomes or waxes on leaves. Accumulation of higher amounts of compatible solutes, amino acids, organic acids, and sugar alcohols are reported as crucial factors to sustain cellular functions during drought periods. Plant growth hormones like abscisic acid also regulate the response of plants towards drought stress (Farooq et al., 2009; Valliyodan and Nguyen, 2006). Several genes, transcription factors or proteins are known to be induced by drought stress and to regulate the abovementioned morphophysiological and biochemical changes in various crop species (Xiang et al., 2007; Cong et al., 2008; JunWei et al., 2006; Tang et al., 2005).

Like in other crop species, a remarkable variation regarding drought tolerance exists among Brassica species. Ashraf and Mehmood (1990) compared drought resistance of different Brassica species and found $B$. napus and $B$. carinata to be the most drought-tolerant and drought-sensitive species, respectively. The crop growth stage also significantly determines the sensitivity of $B$. napus to drought stress (Naderikharaji et al., 2008). Different morphological, biochemical and genetic traits are known as key indicators of drought stress resistance in B. napus. These include possession of deep root system and osmotic adjustment (Mahmood et al., 2004; Kumar and Singh, 1998), accumulation of enzymes (cystein proteinases and acid peroxidases) involved in xylem differentiation and lignification (FernandezGarcia et al., 2011) and increased accumulation of free proline in leaf and stem tissues (Din et al, 2011; Xue et al., 2009). Furthermore, although only very few are well studied, a number of putative genes 
involved in drought and dehydration stress resistance have been identified in B. napus. Examples are the Drought-responsive element binding factor $(D R E B)$ transcription factors which control the expression of several drought inducible genes (Yang et al., 2010) known to be induced by drought (Kagale et al., 2007) and cold stress (Gao et al., 2002; Lindemose et al., 2013). DREBs also regulate chloroplast development and photosynthetic capacity during cold stress (Savitch et al., 2005). The recently identified Calcineurin B-like (CBL) protein interacting calcium-dependent protein kinases (CPKs), called CIPK genes, are also known to be induced by high salinity and osmotic stresses (Chen et al, 2012). Another group of drought induced genes called HD-zip (Homeodomain leucine-zipper) genes are known to be up-regulated by osmotic treatment (Yu et al., 2005). Late embryogenesis abundant (LEA) genes are responsible for enhanced growth and yield under drought stress conditions (Park et al., 2005; Dalal et al., 2009). The abiotic stress related plant hormone ABA, which mediates stomatal closure and developmental changes during water stress (Hartung et al., 1999), is increased by drought stress (Qaderi et al., 2006) but not affected by VL-infection in B. napus (Ratzinger et al., 2009).

When plants are exposed to drought stress in the presence of pathogen stress, they may either benefit or suffer from the complex interactions between the pathogen and the abiotic stress (drought). In general, three possible interactions may be expected. First, drought stress can have a direct effect on pathogen survival. Second, drought by changing host and climatic conditions, may indirectly affect the pathogen potential. And third, drought alone and its interaction with the pathogen can potentially affect the host plant resistance (Desprez-loustau et al., 2006). The effect of drought stress on disease development and host susceptibility seems to be dependent on the pathosystem. On one side, drought may be helpful for the plant if it leads to the so called cross-resistance. Conversely, it may aggravate the disease development or increase host susceptibility to pathogens. For instance, drought stress may enhance resistance of barley to powdery mildew caused by Blumeria graminis f. sp. hordei (Wiese et al., 2004); it also resulted in a significant decrease in Botrytis cinerea infection of tomato (Lycopersicon esculentum)(Achuo et al., 2006), and reduced Verticillium albo-atrum disease symptoms in a highly susceptible alfalfa clone (Pennypacker et al., 1991). Reduced root growth caused by drought stress can reduce the chance of root contact with pathogen propagules in the soil leading to lower incidence of infection. Drought can also activate plant defense pathways (Pertot and Elad, 2012). Foliar pathogens in general tend to have lower infection success under dry conditions (Garrett et al., 2006). However, contradicting results are reported from other pathosystems. Newton and Young (1996) showed that drought causes loss of barley resistance to powdery mildew (Erysiphe graminis f.sp. hordei). It also increased the severity of Phytophthora root rot in tomato (Ristaino and Duniway, 1989). Drought may 
alter host plant physiology favoring pathogen colonization. For example increased concentration of sugars (carbohydrates) and amino acids (proline, asparagine, alanine) in drought stressed forest trees may stimulate growth of different fungal pathogens (Desprez-loustau et al., 2006).

The diverse effects of drought in different pathosystems suggests that studying the significance of drought stress on plant resistance as well as on the extent of disease development particularly for vascular pathogens whose effect mimic drought stress, is very critical. Nevertheless, the effect of drought stress in B. napus - V. longisporum interaction is not yet understood. More importantly, it is not known whether VL-resistance related defence mechanisms or structural changes in the host plant are associated with physiological effects or yield penalties. Understanding the B. napus - V. longisporum pathosystem under drought conditions provides a hint on how to deal with the effects of this host specific pathogen during periods of water deficit. With this background, the present comprehensive study therefore aimed to address the following objectives:

\section{Objectives:}

- to evaluate the level and rate of $V$. longisporum disease development during drought stress periods

- to investigate the effects of $V$. longisporum infection on plant physiology and host response to drought stress

- to study the possible consequences of $B$. napus internal $V$. longisporum resistance mechanisms on plant performance, yield attributes and resistance to drought stress

- to assess the main and interactive effects of VL infection and drought stress factors on plant physiology, agronomic features and expression of drought induced genes

- to verify whether $B$. napus resistance towards $V$. longisporum is stable under drought stress conditions

\subsection{Materials and methods}

\subsubsection{Treatments and experimental conditions}

A factorial pot experiment was installed in a Completely Randomized Design (CRD) under greenhouse conditions and repeated twice. The study consisted of a combination of three experimental factors resulting in 12 treatments. The experimental factors were genotype (two winter oilseed rape genotypes 'Falcon' and 'SEM 05-500256'), disease (mock-inoculated and VL-inoculated) and three watering levels (100, 60 and 30\% field capacity) (Table 4.1). Treatments were arranged in a randomized fashion (Fig. 4.1) 
with three biological replicates each composed of 5 plants grown independently in separate pots. Mockand/or VL-inoculated plants supplied with water at $100 \%$ field capacity were used as control.

Table 4.1 Treatment designation and descriptions

\begin{tabular}{ccccc}
\hline No. & Designation & \multicolumn{2}{c}{ Experimental factors and descriptions } \\
& & Genotype & Disease & Drought \\
\hline 1 & SM1 & SEM 05-500256 & Mock-inoculated & $100 \% \mathrm{FC}$ \\
2 & SM2 & SEM 05-500256 & Mock-inoculated & $60 \% \mathrm{FC}$ \\
3 & SM3 & SEM 05-500256 & Mock-inoculated & $30 \% \mathrm{FC}$ \\
4 & SV1 & SEM 05-500256 & VL-inoculated & $100 \% \mathrm{FC}$ \\
5 & SV2 & SEM 05-500256 & VL-inoculated & $60 \% \mathrm{FC}$ \\
6 & SV3 & SEM 05-500256 & VL-inoculated & $30 \% \mathrm{FC}$ \\
7 & FM1 & Falcon & Mock-inoculated & $100 \% \mathrm{FC}$ \\
8 & FM2 & Falcon & Mock-inoculated & $60 \% \mathrm{FC}$ \\
9 & FM3 & Falcon & Mock-inoculated & $30 \% \mathrm{FC}$ \\
10 & FV1 & Falcon & VL-inoculated & $100 \% \mathrm{FC}$ \\
11 & FV2 & Falcon & VL-inoculated & $60 \% \mathrm{FC}$ \\
12 & FV3 & Falcon & VL-inoculated & $30 \% \mathrm{FC}$ \\
\hline
\end{tabular}

FC, Field capacity. $100 \%$ FC, maximum watering. $60 \%$ FC, moderate drought stress. $30 \% \mathrm{FC}$, severe drought stress.

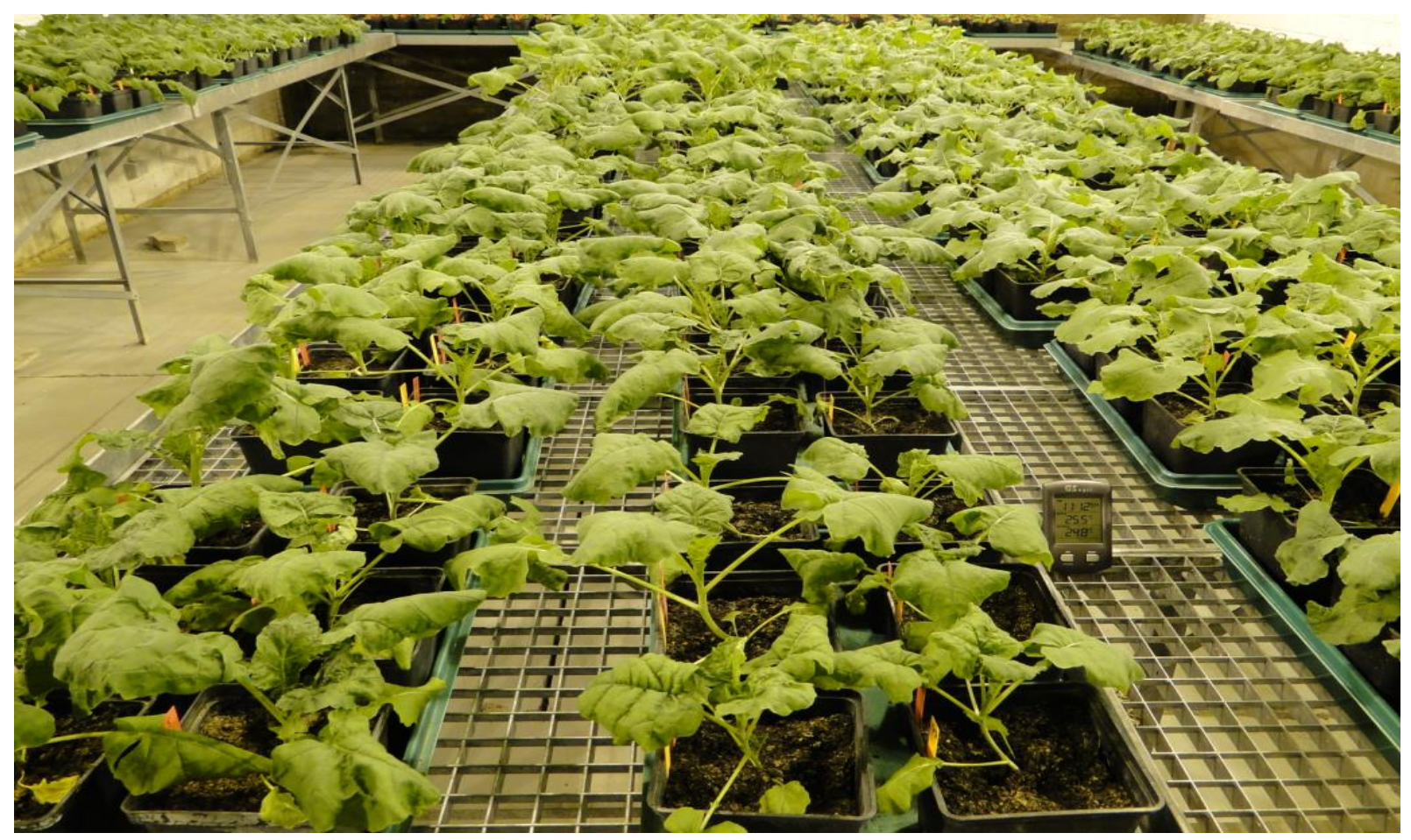

Figure 4.1 Partial view of experimental arrangement in a completely randomized design in the greenhouse experiment (14 DPI). 


\subsubsection{Determination of field capacity in the soil substrate}

A substrate composed of commercial soil (Fruhstorfer Erde, Type; pH 6.0; Hawita-Gruppe, Vechta, Germany) and river sand in a 3:1 ratio was used. For determination of substrate gravimetric moisture content at field capacity the method described in Grzesiak et al. (2013) and Somasegaran and Hoben (1985) was adopted. Briefly, plastic cylinders $(2.5 \mathrm{~cm}$ radius and $20 \mathrm{~cm}$ height) with a drainage plastic net at the bottom were filled with gravel $(20-50 \mathrm{~mm}$ diameter) up to $3 \mathrm{~cm}$ in six replications and placed for $15 \mathrm{~min}$ in a container filled with tap water. Cylinders were then covered with aluminum foil and placed on a wire grid for $15 \mathrm{~min}$ to drain the excess water. Subsequently their weight was measured using an analytical balance $\left(\mathrm{Wt}_{1}\right)$. Then, $150 \mathrm{~g}$ of oven dried $\left(105^{\circ} \mathrm{C}\right.$ for 24 hours) substrate was added to each cylinder and cylinders were placed in the water container for two hours. Additional water was gently poured on the surface of the substrate until it was fully saturated. After saturation, cylinders were removed from the water container and allowed to stand on a wire grid for two hours to drain the excess water through holes in the bottom of the cylinders (until drainage equilibrium) with the top covered to prevent evaporation. Cylinders were then placed on absorbent paper for $15 \mathrm{~min}$ to drain the excess water held in the spaces between gravel particles and then a second weight was recorded $\left(\mathrm{Wt}_{2}\right)$. Moisture content of the substrate at this stage was assumed to be equal to field capacity and calculated as $\mathrm{FC}_{100}=\left[\left(\mathrm{Wt}_{2}\right.\right.$ - substrate oven dry weight $\left.\left.)-\mathrm{Wt}_{1}\right)\right]$. This water status was considered as moisture content at field capacity $\left(\mathrm{FC}_{100}\right)$ and was maintained throughout the experiment period for control treatments. Accordingly, 60 and $30 \%$ of the water content at $\mathrm{FC}_{100}$ were used to make $60 \%\left(\mathrm{FC}_{60}\right)$ and $30 \%\left(\mathrm{FC}_{30}\right)$ field capacity treatments, respectively (Abedi and Pakniyat , 2010).

\subsubsection{Plant material and growing conditions}

Two winter oilseed rape genotypes expressing contrasting levels of resistance against $V$. longisporum were used. Seed of the VL- susceptible commercial winter oilseed rape cultivar Falcon was purchased from Norddeutsche Pflanzenzucht Hans-Georg Lembke KG (NPZ, Hohenlieth, Germany). The VL-tolerant B. napus accession SEM 05-500256 seed was obtained from Lantmännen SW Seed (Svalöv, Sweden). Seeds of both genotypes were double surface sterilized with $70 \%$ ethanol for two minutes under constant shaking and rinsed with autoclaved tap water. Seeds were then sown in multi-pot trays containing autoclaved silica sand and soil $(1: 1)$ and grown in a climate-controlled chamber $\left(20^{\circ} \mathrm{C}\right.$ temperature, 16 hours light and 60\% relative humidity). Two weeks after sowing (BBCH 12), seedlings were subjected to vernalization for 13 weeks by gradually lowering the temperature and day length to $4^{\circ} \mathrm{C}$ and 10 hours, respectively. Before seedlings were inoculated with VL, acclimatization was performed for three days by keeping vernalized seedlings under $10^{\circ} \mathrm{C}$ and 16 hours light conditions. After 
inoculation, plants were transplanted to plastic pots (1.5 liter) filled with a substrate composed of commercial soil (section 4.2.2) and river sand in a 3:1 ratio and grown under greenhouse conditions. Average climatic conditions during the whole experimental period in the greenhouse were 16 hours light, $24^{\circ} \mathrm{C}$ temperature and $70 \%$ relative humidity. All other agronomic practices such as fertilization (Hakaphos COMPO, Germany) were equally applied to all treatments according to the manufacturer's recommendations.

\subsubsection{Fungal isolate and inoculation procedures}

Verticillium longisporum isolate VL43 (Fig. 1.2), originally isolated from B. napus in Northern Germany and highly virulent on OSR (Zeise and Tiedemann, 2001) was used. For inoculum preparation, 500ul of VL43 spore stock solution (VL conidial suspensions in PEGB supplemented with $25 \%$ glycerol and stored at $-80^{\circ} \mathrm{C}$ ) was added to $250 \mathrm{ml}$ of PEGB and propagated for 10 days at $23^{\circ} \mathrm{C}$ in the dark with constant shaking. The culture was subsequently filtered through sterile gauze and spore concentration was adjusted to $10^{6}$ conidia/ml using a haemocytometer (Thoma chamber, $0.100 \mathrm{~mm}$ depth, Germany). Inoculation was performed following a standard cut-root dip inoculation technique where roots of vernalized seedlings ( $\mathrm{BBCH}$ 14) were washed, injured by cutting the tips with a sterile scissor, and submerged in the spore suspension for $30 \mathrm{~min}$. Control plants were dipped in the same way using autoclaved tap water. After inoculation, seedlings were transplanted into plastic pots and grown in the greenhouse under standardised conditions as described in section 4.2.3.

\subsubsection{Determination of soil water content and maintenance of water regime}

The amount of water to be supplied to plants in each treatment was calculated considering the initial pot weight at different field capacity levels and plant biomass increment as well (Earl, 2003; Champolivier and Merrien, 1996; Grzesiak et al., 2013). Initial pot weight in each treatment was determined from the weight of substrate $(1 \mathrm{~kg})$, amount of water required to make up the different field capacities (considering initial substrate moisture content) and plant weight. To determine the weekly plant biomass increment, 15 randomly selected plants from each treatment were carefully uprooted, roots were washed under running tap water to remove adhering soil particles and the total fresh weight was determined. During the first three weeks after inoculation (until $21 \mathrm{DPI}$ ), all plants were uniformly supplied with water at $\mathrm{FC}_{100}$. For drought stress treatments, watering at $\mathrm{FC}_{60}$ and $\mathrm{FC}_{30}$ was started at 21 DPI when a reasonable acclimatization of transplanted seedlings, successful colonization of roots by the pathogen and sufficient extent of resistance responses were attained (Eynck et al., 2009b). The water 
consumption in each pot was daily monitored by weighing the pots. Re-watering was done to restore the soil water content according to the individual field capacity treatment.

\subsubsection{Plant sample collection and preservation}

For non-destructive measurements (disease severity, physiological and agronomic data), a total of 30 plants per treatment, 15 VL-inoculated and 15 mock-inoculated, each representing three biological replicates consisting of 5 plants, were used. Similar numbers of plants from each treatment were weekly harvested for biochemical and molecular analyses involving destructive sampling. Leaf and hypocotyl samples were frozen in liquid nitrogen immediately after harvest and stored at $-80^{\circ} \mathrm{C}$ until further analysis.

\subsubsection{Disease assessment}

\subsubsection{Area Under Disease Progress Curve}

Disease evaluation was performed using an assessment key with nine classes (Table 4.2). AUDPC values were calculated from weekly disease severity recordings using the formula:

AUDPC $=\sum_{\mathrm{t}=1}^{n-1}\left[\frac{\left(y_{i}+y_{i+1}\right)}{2}\right]\left[\left(t_{i+1}-t_{i}\right)\right]$ Where $y_{i}=$ disease severity at the $i^{t h}$ observation, $t_{i}=$ the time (days after inoculation) at the $i^{\text {th }}$ observation and $n=$ total number of observations (Campbell and

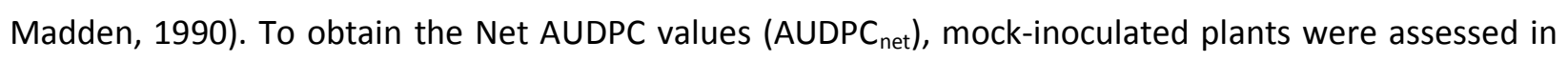
the same way for natural (age-mediated) yellowing and senescence ( UUDPC $_{\text {mock }}$ ) and this value was subtracted from corresponding VL-inoculated treatments $\left(A U D P C_{\text {inoc }}\right)$. That is $A_{U D P C}$ net $=A U D P C_{\text {inoc }}$ $\mathrm{AUDPC}_{\text {mock }}$ (Eynck et al., 2009b).

\subsubsection{Relative stunting}

Stunted growth is one of the typical greenhouse symptoms observed in susceptible OSR plants infected with $V$. longisporum. To evaluate this effect, plant height (from the base of hypocotyl to the tip of the central main shoot) was weekly measured with a ruler. Relative stunting (RS) referring to the reduction of plant height in inoculated plants $\left(h_{\text {inoc }}\right)$ relative to plant height of mock-inoculated $\left(h_{\text {mock }}\right)$ plants was calculated as RS $(\%)=100-\left[\left(h_{\text {inoc }} \times 100\right) /\left(h_{\text {mock }}\right)\right]$. 
Table 4.2 Disease scoring index for assessment of Verticillium longisporum disease severity in Brassica napus (modified from Zeise, 1992).

\begin{tabular}{cl}
\hline Index & \multicolumn{1}{c}{ Disease symptom } \\
1 & Healthy plants with no symptoms \\
2 & Up to $10 \%$ of leaves on the main stem show either yellowing and/or black veins or are dead \\
3 & $11-20 \%$ of leaves on the main stem show either yellowing and/or black veins or are dead \\
4 & $21-40 \%$ of leaves on the main stem show either yellowing and/or black veins or are dead \\
5 & $41-60 \%$ of leaves on the main stem show either yellowing and/or black veins or are dead \\
6 & $61-80 \%$ of leaves on the main stem show either yellowing and/or black veins or are dead \\
7 & $81-100 \%$ of leaves on the main stem show either yellowing and/or black veins or are dead \\
8 & Only the terminal bud (apex) is still alive \\
9 & The plant is dead
\end{tabular}

\subsubsection{RT PCR based quantification of $V$. longisporum infection}

\section{DNA extraction}

A modified CTAB DNA extraction protocol (Brandfass and Karlovsky, 2008) was used for extraction of genomic DNA from hypocotyl tissue. Briefly, hypocotyl samples from VL- and mock-inoculated plants were collected at 28 and 49 DPI as described in section 4.2.4, immediately frozen in liquid nitrogen and stored at $-80^{\circ} \mathrm{C}$ until further analysis. Hypocotyl samples were lyophilized for $72 \mathrm{~h}$ (freeze dryer ALPHA 14, Martin Christ Gefriertrocknungsanlagen $\mathrm{GmbH}$, Osterode am Harz, Germany) and grinded with a mixer mill (MM 200, Retsch GmbH, Haan, Germany). Subsequently, 100mg of hypocotyl powder was transferred to $2 \mathrm{ml}$ tubes and $1 \mathrm{ml}$ of CTAB buffer, $1 \mu \mathrm{L}$ of proteinase $\mathrm{K}(20 \mathrm{mg} / \mathrm{mL})$, and $2 \mu \mathrm{L}$ mercaptoethanol were added and the sample was mixed by vortexing. Then $3 \mu \mathrm{L}$ of RNase $A(10 \mathrm{mg} / \mathrm{ml})$ solution was added and the sample was incubated at $37^{\circ} \mathrm{C}$ for $15 \mathrm{~min}$. After chloroform-isoamyl alcohol extraction, the sample was centrifuged $(16,060 \times \mathrm{g})$ and the upper aqueous phase $(600 \mu \mathrm{L})$ was transferred to $1.5 \mathrm{~mL}$ tubes containing $194 \mu \mathrm{L}$ of $30 \%$ PEG solution and $100 \mu \mathrm{L}$ of $5 \mathrm{M} \mathrm{NaCl}$. The DNA was pelleted by centrifugation $(16,060 \times \mathrm{g})$, washed twice with $70 \%$ ethanol and let to dissolve overnight in $200 \mu \mathrm{l}$ of TE buffer at $4^{\circ} \mathrm{C}$. The quality and concentration of DNA was examined by loading $5 \mu \mathrm{L}$ of DNA sample on 1\% (w/v) agarose gel (Agarose low EEO, AppliChem GmbH, Germany) prepared in TBE buffer. Electrophoresis was carried out at $3 \mathrm{~V} / \mathrm{cm}$ for $60 \mathrm{~min}$ and gel was visualized by ethidium bromide staining and documented using a digital imaging system (Bio-Rad Gel Doc 1000). Using DNA of bacteriophage Lambda as a standard, concentration of DNA was quantified by densitometry using 
Quantity One ${ }^{\circledast}$ software (Version 4.5.0 Bio-Rad Laboratories). DNA samples were stored at $-20^{\circ} \mathrm{C}$ until qPCR analysis.

\section{Preparation of VL DNA standard for qPCR}

Total genomic DNA of $V$. longisporum was extracted from isolate VL43 grown on potato extract glucose broth following the protocol described in section 2.2.4. Briefly, fungal mycelium was filtered through filter paper, freeze dried and then crushed in liquid nitrogen. Subsequently, DNA was extracted using a modified CTAB DNA extraction protocol (Brandfass and Karlovsky, 2008). Concentration of DNA was quantified as described in section 4.2.7.4 and a dilution series of 1 ng to $0.001 \mathrm{pg}$ of $V$. longisporum DNA with a dilution factor of 10 was produced in $1 X$ TE buffer.

\section{Quantitative real-time PCR (qPCR)}

In this procedure, a highly species specific primer pair developed for quantification of $V$. longisporum infection in OSR (Knüfer, 2013) was used. Using these primers (sense primer OLG70 5'CAGCGAAACGCGATATGTAG 3' and antisense primer OLG71 5'GGCTTGTA GGGGTTTAGA 3') which amplify a $261 \mathrm{bp}$ fragment of the ITS region, a real time PCR was performed using ABsolute Blue SYBR Green Fluorescein Mix (ABgene, Hamburg, Germany) in a CFX384 real-time PCR detection system (BioRad laboratories, Inc). Each RT-qPCR reaction had 5-10ng of template DNA, $5 \mu$ l of ABsolute Blue QPCR SYBR Green Fluorescein Mix, $0.3 \mu \mathrm{M}$ of forward and reverse primers and $\mathrm{dd}_{2} \mathrm{O}$ to give a final volume of $10 \mu \mathrm{l}$. The amplification protocol was as follows: initial enzyme activation step $15 \mathrm{~min}$ at $95^{\circ} \mathrm{C}$ and 40 cycles of $95^{\circ} \mathrm{C}$ for $10 \mathrm{~s}$ (denaturation), $60^{\circ} \mathrm{C}$ for $15 \mathrm{~s}$ (primer annealing) and $72^{\circ} \mathrm{C}$ for $15 \mathrm{~s}$ (extension). Reaction was completed with a final elongation step of $72^{\circ} \mathrm{C}$ for $2 \mathrm{~min}$. For evaluation of the amplification specificity, a melting curve analysis $\left(55^{\circ} \mathrm{C}\right.$ to $\left.95^{\circ} \mathrm{C}\right)$ with a heating rate of $0.5^{\circ} \mathrm{C} / 5 \mathrm{~s}$ was used. PCR for all treatment samples were performed with three biological and technical replicates and data was analyzed using CFX Manager Software (Bio-Rad laboratories, Inc).

\subsubsection{Physiological data}

\subsubsection{Gas exchange}

The leaf gas exchange parameters, photosynthesis rate $(A)$, transpiration rate $(E)$ and stomatal conductance (gs), were measured using a portable infrared gas analyzer LCpro+ (ADC BioScientific Ltd., Hertfordshire, UK) equipped with a broad leaf chamber and laser-trimmed humidity sensor. Measurement was performed in situ from the upper $3^{\text {rd }}$ fully developed leaves at 28 and $49 \mathrm{DPI}$ (one and four weeks after the beginning of the drought stress treatment, respectively) representing the beginning of flowering and fruit development stages, respectively. Conditions during measurement were as 
follows: projected leaf surface area was $6.25 \mathrm{~cm}^{2}$; PAR (Photosynthetically Active Radiation) at the leaf surface was set to $700 \mu \mathrm{mol} \mathrm{m} \mathrm{m}^{-2}$; and relative humidity and $\mathrm{CO}_{2}$ concentration were set to ambient greenhouse conditions, $70 \%$ and $500 \mu \mathrm{mol} \mathrm{mol}^{-1}$, respectively. Each parameter was measured from 5 independently grown individual plants from each treatment. Water use efficiency (WUE) was computed as the ratio of photosynthesis to transpiration rate.

\subsubsection{Relative water content}

Leaf relative water content (RWC) was determined gravimetrically from the $3^{\text {rd }}$ upper fully expanded leaves (Mationn et al., 1989). Briefly, leaf discs $\left(\approx 10 \mathrm{~cm}^{2}\right)$ were excised (excluding the midrib) and fresh weight (FW) was immediately determined on an analytical balance. Subsequently, leaves were immersed in double distilled water in petri dishes for three hours in the dark and the turgid weight (TW) was taken after blotting. Thereafter, dry weight (DW) was obtained after oven drying of the leaves overnight at $70^{\circ} \mathrm{C}$. Percent leaf relative water content was calculated using the formula: RWC $=[(F W-$ DW) / (TW-DW)] x 100. For each replication, measurement was done from five leaf samples taken from five independently grown plants.

\subsubsection{Proline content}

Accumulation of free proline content in leaf and hypocotyl samples was determined according to the method of Bates et al., 1973. In this procedure, 100mg of leaf or hypocotyl milled samples prepared as described in section 2.2.7.4 were homogenized in $2 \mathrm{ml}$ of $3 \%$ sulfosalicylic acid. After centrifugation

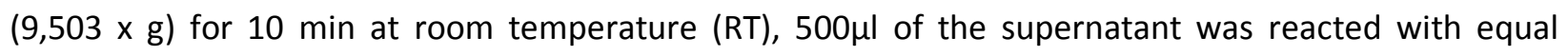
volumes of acid-ninhydrin solution and glacial acetic acid in a water bath for $1 \mathrm{~h}$ at $100^{\circ} \mathrm{C}$. Known concentrations of L-proline (Fluka Biochemika) ranging between $150-0.5 \mu \mathrm{g} / \mathrm{ml}$ were prepared in $3 \%$ aqueous sulfosalicylic acid and treated the same way as plant extract samples. The reaction was terminated by placing tubes on ice and proline was extracted by adding $800 \mu$ l of toluene into $1,200 \mu l$ of the reaction mixture. Subsequently, $200 \mu$ l of the chromospheres containing toluene was loaded on 96well microtitre plate and absorbance was read in triplicates at $520 \mathrm{~nm}$ using a Microplate Spectrophotometer ( $\mu$ Quant, Bio-Tek Instruments, Bad Friedrichshall, Germany). Toluene was used as a blank and concentration of proline in plant samples was estimated by referring to the standard curve produced from known concentrations of L-proline. Finally, $\mu$ moles of proline per gram of leaf and hypocotyl dry weight samples were calculated using the formula: [( $\mu \mathrm{g}$ proline/ml $\times \mathrm{ml}$ toluene) /

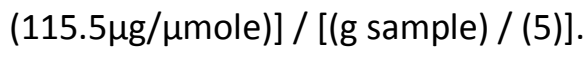




\subsubsection{Measurement of yield and agronomic variables}

In order to assess the magnitude of yield reduction and changes in morphological traits due to VLinfection under drought stress conditions, several agronomic parameters were measured. Production of side shoots was assessed by counting the number of primary branches per plant. Growth rate was determined by evaluating phenological growth stages according to the $\mathrm{BBCH}$ scale (Appendix 4.13). Days to $50 \%$ flowering were calculated as the number of days (DPI) required for $50 \%$ or more plants in a treatment to reach growth stage $\mathrm{BBCH}$ 65. Assessments of biomass yield and hypocotyl thickness were performed at the end of the experiment (49 DPI). Stem diameter at the tip of hypocotyl was measured using a digital calliper. Root fresh weight was estimated after removing soil and water adhering to the root surface by washing and blot drying. For determination of shoot fresh biomass yield, the whole portion of the above-ground plant part (above the hypocotyl) was weighed immediately after harvest. To determine dry biomass yield, root and shoot samples were oven dried $\left(70^{\circ} \mathrm{C}\right.$ for $\left.24 \mathrm{~h}\right)$ and weighed separately. Total fresh and dry matter was expressed as the sum of root and shoot biomass weights.

\subsubsection{Expression of drought responsive genes}

Upon infection with $V$. longisporum, resistant OSR plants respond by increased lignification and accumulation of vascular occlusions (Eynck et al., 2009b). These histological changes in vascular tissue may interfere with water and nutrient transport and might make plants more sensitive to drought stress. In order to verify this hypothesis, expression of drought responsive genes (DR-genes) under optimum watering and severe drought stress as well as VL-infection conditions were investigated.

\subsubsection{Primer sequence of studied genes}

Genes that show contrasting expression levels and encode for functional and regulatory proteins involved in drought stress tolerance and response of B. napus were selected from previous studies. Gene specific primers (Table 4.3) which amplify partial sequences of the selected genes were either taken from the literature or designed using primer3 software (http://www.ncbi.nlm.nih.gov/tools/primer-blast).

\subsubsection{PCR amplification and analysis of PCR products}

Optimization of primer annealing temperatures and concentration of PCR components were carried out by running a gradient PCR using a T GRADIENT Thermocycler (Biometra, Göttingen, Germany). After optimization, all genes were amplified in a $25 \mu$ l total reaction volume composed of 10x PCR buffer, $2.5 \mathrm{mM}$ of each dNTPs, $50 \mathrm{mM} \mathrm{MgCl} 2,1$ unit of BioTaq Taq DNA polymerase (Bioline $\mathrm{GmbH}$, Luckenwalde, Germany), $25 \mathrm{pmol}$ of each primer and 10ng of template DNA. Amplification was performed by heating samples at $95^{\circ} \mathrm{C}$ for $2 \mathrm{~min}$, followed by 34 cycles each consisting of denaturation at $95^{\circ} \mathrm{C}$ for $2 \mathrm{~min}$, 
annealing at $64^{\circ} \mathrm{C}$ for $1 \mathrm{~min}$ and elongation at $72^{\circ} \mathrm{C}$ for $1 \mathrm{~min}$. The reaction was terminated with a final extension step of $10 \mathrm{~min}$ at $72^{\circ} \mathrm{C}$. In order to insure reproducibility, reaction was repeated trice. To verify the amplicon size of each gene, PCR products were electrophoresed in 1.2\% (W/V) TBE-agarose gel, stained with ethidium bromide and visualized under UV light as described in section 2.2.7.4. In addition to PCR amplification, specificity of amplification was further confirmed by cloning and sequence analysis of partial DR gene fragments.

Table 4.3 Sequences of primer pairs used in RT-PCR

\begin{tabular}{|c|c|c|}
\hline Gene name & Primer sequence & Source \\
\hline \multirow{2}{*}{ BnActin } & 5'- GCCCAGAAGTCTTGTTCCAG-3' (F) & \multirow{2}{*}{ Lee et al., 2008} \\
\hline & $5^{\prime}-$ GAACCACCGATCCAGACACT-3' (R) & \\
\hline \multirow{2}{*}{$B n C B F 17$} & 5'- AATGATCATGGCATGAACATGGC-3' (F) & \multirow{2}{*}{ Gao et al., 2002} \\
\hline & 5'- ACTCCTCGTCCATGTAAAACCC-3' (R) & \\
\hline \multirow{2}{*}{$B n C I P K 1$} & 5'- GACCGTGGAAAGGACTCCATGAAGCATC-3’ (F) & \multirow{2}{*}{ This study } \\
\hline & 5'- TCTGGCGGCTTCCAGATGATGT-3’ (R) & \\
\hline \multirow{2}{*}{ DREB2-23 } & 5'- GAGCTGTCCGAAGAAACCTG-3’ (F) & \multirow{2}{*}{ This study } \\
\hline & 5'- ATAACTCCAAAGGGACACGTC-3’ (R) & \\
\hline \multirow{2}{*}{ BnHB6 } & 5'- ATGATGAAGAGATTAAGCAGTTCAGA-3’ (F) & \multirow{2}{*}{ Yu et al., 2005} \\
\hline & 5'- GTGATCCTCCGTCTGCTCCA-3' (R) & \\
\hline \multirow[t]{2}{*}{ BnP5CS1 } & 5'-CGATTTGGACTTGGTGCTGA-3' (F) & \multirow[t]{2}{*}{ Xue et al., 2009} \\
\hline & 5'-GCCCATCCTCTCCTAGTCTC-3' (R) & \\
\hline \multirow[t]{2}{*}{ BnP5CS2 } & 5'-ССАТТАТСТТССТССТСТСАС-3' (F) & \multirow[t]{2}{*}{ Xue et al., 2009} \\
\hline & 5'-AACAACTGCTGTCCCAA CC-3' (R) & \\
\hline \multirow{2}{*}{$B n L E A 4-1$} & 5'-ATGCAGTCGATGAAGGAAACAGC-3' (F) & \multirow{2}{*}{ This study } \\
\hline & 5'-CCGGCGGAGGATATACTGGATAA-3' (R) & \\
\hline \multirow[t]{2}{*}{ ME-leaN4 } & 5'-GGCAAGGACAAGACTTCCCA-3' (F) & \multirow[t]{2}{*}{ Park et al., 2005} \\
\hline & 5'-CGGATCAGTGCTCTGAGTAG-3' (R) & \\
\hline
\end{tabular}

\subsubsection{Isolation and cloning of drought induced genes}

\section{Purification of PCR products from gel}

PCR products of DR-gene partial DNA fragments were generated using a high fidelity DNA polymerase (Pfu DNA polymerase recombinant, Fermentas) which exhibits $3^{\prime}$ to 5'proof reading activity that enable the correction of nucleotide incorporation errors. PCR was conducted in $25 \mu$ l reaction volume containing 10ng template DNA, 10X Pfu buffer with $\mathrm{MgSO}_{4}$, dNTP mix $(0.2 \mathrm{mM}$ each), 25 pmol of each primer, and 0.5 unit of recombinant Pfu DNA polymerase (Fermentas). A similar PCR program as described in 4.2.10.2 was used for amplification. After examining the PCR products on 1\% low melting point agarose gel in 1X TAE buffer, PCR products were purified by extracting DNA fragments from a gel. Briefly, the amplicon was excised from the gel without exposing to UV and immediately weighed and 
dissolved in 3 volumes (V/W) of DNA-binding buffer (6M NaCl, 50mM Tris pH 7.6, 10mM EDTA pH 8.0) for $10 \mathrm{~min}$ at $50^{\circ} \mathrm{C}$. Then, $6 \mu \mathrm{l}$ of glass milk was added and the mixture was incubated for $10 \mathrm{~min}$ at RT with constant mixing. The matrix was then pelleted by centrifugation at $16,060 \times \mathrm{g}$ for $30 \mathrm{~s}$. After repeating the DNA binding step by adding $500 \mu$ l DNA binding buffer, the pellet was washed twice by resuspending in $150 \mu \mathrm{l}$ of washing buffer $(400 \mathrm{mM} \mathrm{NaCl}, 20 \mathrm{mM}$ Tris pH 8.0, 2mM EDTA pH 8.0 and 50\% absolute EtOH v/v). Finally, DNA was eluted from glass milk by adding $20 \mu$ of $\mathrm{dd}_{2} \mathrm{O}$ water and incubation for $5 \mathrm{~min}$ at $50^{\circ} \mathrm{C}$.

\section{Preparation of a cloning vector}

Pfu DNA polymerase generates a blunt-ended PCR fragment which requires a linearized blunt-ended vector for cloning. Thus, a standard cloning vector pBluescript $\mathrm{SK}^{-}$was linearized via digestion with a restriction enzyme Smal (Fermentas) that generates a blunt-ended product. Digestion reaction was set by mixing $5 \mu \mathrm{g}$ of pBluescript SK', $1 \mathrm{X}$ buffer Tango (Fermentas), 25 unit of Smal (Fermentas) and dd $\mathrm{d}_{2} \mathrm{O}$ to produce a final volume to $50 \mu \mathrm{l}$. The reaction was performed by incubation at $30^{\circ} \mathrm{C}$ for $1 \mathrm{~h}$ followed by heat inactivation of the enzyme at $65^{\circ} \mathrm{C}$ for $20 \mathrm{~min}$. To prevent self-ligation (self-circularization), the linearized vector was dephosphorylated prior to ligation with Calf Intestinal Alkaline Phosphatase (CIAP, Invitrogen) following the manufacturer's protocol. Removal of CIAP from linearized dephosphorylated vector was done by phenol extraction.

\section{Ligation}

Purified PCR products were cloned to pBluescript SK vector by setting up a ligation reaction in $0.5 \mathrm{ml}$ microcentrifuge tubes known to have low DNA binding capacity. The ligation reaction cocktail consisted of a 3:1 molar ratio mixture of vector and insert DNA, $2 \mu$ l of 10x T4 DNA ligase buffer, $2 \mu \mathrm{l} 6 \mathrm{mM}$ rATP, $2 \mu \mathrm{l}$ of $50 \%$ PEG 4000 solution, 5 units T4 DNA ligase (Fermentas) and nuclease-free water to make the final volume $20 \mu \mathrm{l}$. The reaction was performed by placing reaction tubes in a water bath at RT for $30 \mathrm{~min}$ followed by overnight incubation at $4^{\circ} \mathrm{C}$.

\section{Preparation of DH5-alpha competent cells}

Preparation of DH5-alpha Escherichia coli competent cells and transformation procedures were performed according to the modified method of Inoue et al., 1990. Briefly, a starter culture was prepared by growing a single colony bacterial culture in SOB medium for $8 \mathrm{~h}$ at $37^{\circ} \mathrm{C}$ with vigorous shaking at $8.6 \mathrm{x}$. Then, $2 \mathrm{ml}$ of the starter culture was transferred to $250 \mathrm{ml}$ of SOB medium and grown overnight at $18^{\circ} \mathrm{C}$ with moderate shaking at $2.1 \times \mathrm{g}$. When the $\mathrm{OD}_{600}$ reached 0.6 , the culture was transferred to an ice bath for $10 \mathrm{~min}$. Cells were then harvested by centrifugation $\left(1520 \times \mathrm{g} ; 10 \mathrm{~min} ; 4^{\circ} \mathrm{C}\right)$ 
and gently resuspended in $20 \mathrm{ml}$ of ice-cold Inoue transformation buffer $\left(55 \mathrm{mM} \mathrm{MnCl} 2.4 \mathrm{H}_{2} \mathrm{O}, 15 \mathrm{mM}\right.$ $\mathrm{CaCl}_{2} \cdot 2 \mathrm{H}_{2} \mathrm{O}$ and $250 \mathrm{mM} \mathrm{KCl}$ and $10 \mathrm{mM}$ PIPES; $\mathrm{pH}$ 6.7). Subsequently, $1.5 \mathrm{ml}$ of DMSO was added and the bacterial suspension was incubated on ice for $10 \mathrm{~min}$. After incubation, 100 $\mathrm{\mu l}$ aliquots of the bacterial suspension were dispensed into chilled sterile $1.5 \mathrm{ml}$ microcentrifuge tubes, immediately snap-frozen in liquid nitrogen and stored at $-80^{\circ} \mathrm{C}$ until used for transformation.

\section{Transformation}

Transformation was performed by mixing $100 \mu$ l aliquots of $E$. coli DH5- $\alpha$ competent cell with $2 \mu l$ of the ligation product. After incubation on ice for $30 \mathrm{~min}$, cells were heat shocked $\left(42^{\circ} \mathrm{C}\right)$ for $40 \mathrm{~s}$ and immediately placed on ice for 2 min. Afterwards, $900 \mu$ l of SOC medium was added and cells were incubated at $37^{\circ} \mathrm{C}$ for $90 \mathrm{~min}$ with constant shaking. $200 \mu \mathrm{l}$ volumes of the transformed competent cells were then transferred onto MacConkey agar plates amended with additional lactose $(10 \mathrm{~g} / \mathrm{liter})$ and ampicillin $(100 \mu \mathrm{g} / \mathrm{ml})$. After $12-16 \mathrm{~h}$ of incubation at $37^{\circ} \mathrm{C}$, the transformant cells harboring recombinant plasmids were counter selected from false positives based on colony color and further confirmed via colony PCR. Transformed cells were preserved at $-80^{\circ} \mathrm{C}$ in $10 \%$ glycerol.

\section{Plasmid miniprep}

Extraction of plasmid DNA from transformant E. coli DH5- $\alpha$ competent cells was performed following the alkaline lysis procedure developed by Birnboim \& Doly (1979). For this procedure, a bacterial culture was prepared from single colony transformant cells in LB medium supplied with ampicillin (100 $\mathrm{gg} / \mathrm{ml})$. Two $\mathrm{ml}$ of overnight bacterial culture grown at $37^{\circ} \mathrm{C}$ under constant shaking was transferred to microcentrifuge tubes and cells were harvested by centrifugation (6,082 x g for $5 \mathrm{~min})$. Cells were then resuspended in $200 \mu$ lice cold GLC buffer (50mM Tris pH 8.0, 50mM glucose, and 10mM Na-EDTA), 2.4 $\mu \mathrm{l}$ lysozyme $(40 \mathrm{mg} / \mathrm{ml})$ and $3 \mu \mathrm{l}$ RNase $\mathrm{A}(10 \mathrm{mg} / \mathrm{ml})$ and incubated for $15 \mathrm{~min}$ at RT. Subsequently, $300 \mu \mathrm{l}$ LYZ buffer (1\% SDS and $0.2 \mathrm{~N} \mathrm{NaOH}$ ) was added and contents of the mixture were further incubated for $10 \mathrm{~min}$ at RT followed by another $30 \mathrm{~min}$ incubation after addition of HS buffer (29.4 g of potassium acetate and $3.8 \mathrm{ml}$ of formic acid in $100 \mathrm{ml} \mathrm{H}_{2} \mathrm{O}$ ). Next, cell debris were separated by centrifugation $\left(16,060 \times \mathrm{g}\right.$ at $\left.4^{\circ} \mathrm{C}\right)$ and the supernatant containing the plasmid DNA was transferred to new tubes, mixed with 0.65 volume of isopropanol and incubated for $10 \mathrm{~min}$ at RT. Then, DNA was precipitated by centrifugation $\left(16,060 \times \mathrm{g}\right.$ at $\left.4^{\circ} \mathrm{C}\right)$ and washing twice with $70 \%$ ethanol. Finally, the DNA pellet was dried in a speed-vac for $10 \mathrm{~min}$ at $30^{\circ} \mathrm{C}$ and resuspended in $50 \mu \mathrm{l}$ of $1 \mathrm{X}$ TE buffer. The presence of the correct insert in plasmid DNA was verified by colony PCR and PVUII (Fermentas) restriction digest analysis. Prior 
to sequencing, purification of miniprep plasmid DNA samples was performed using SureClean (Bioline $\mathrm{GmbH}$, Germany) kit following manufacture's protocol.

\subsubsection{DNA sequencing and sequence analysis}

Purified plasmid clones were sequenced using the universal 77 promoter forward and reverse primers at Eurofins MWG Operon (Ebersberg, Germany). Resulting sequences were vector-clipped and nucleotide sequence identity was verified by comparison with known gene sequences available in the NCBI GenBank database using Blastn search (http://blast.ncbi.nlm.nih.gov).

\subsubsection{Real time quantitative PCR analysis}

\section{RNA extraction and reverse transcription}

RNA was isolated from leaf samples collected 7 days after drought stress treatment (28 DPI), where the effect of drought stress was visible as evidenced by physiological data. Leaf samples collected from normally watered $(100 \% \mathrm{FC})$ and severely stressed $(30 \% \mathrm{FC})$ plants were ground in liquid nitrogen and total RNA was isolated from 100mg tissue using Tri-Reagent (Sigma) according to the manufacturer's protocol. Following extraction, RNA integration was analyzed on $1 \%(w / v)$ formaldehyde-agarose gel. RNA quality and concentration were measured using an Epoch microplate spectrophotometer (Epoch; BioTek Inc., Winooski, VT, USA). cDNA was synthesized from 1ug of total RNA samples using the QuantiTect Reverse Transcription Kit (QIAGEN) and following the manufacturer's instructions. Before reverse transcription, any genomic DNA contamination was removed with gDNA Wipeout Buffer following the protocol. Furthermore, further check for genomic DNA contamination of RNA samples was done by running a PCR using BnActin gene primer and RNA template.

\section{Real time PCR}

Quantitative RT-PCR was performed using ABsolute Blue SYBR Green Fluorescein Mix (ABgene, Hamburg, Germany) following manufacturer's recommendations. PCR reaction was set up in $10 \mu$ l total reaction volume consisting of $5 \mu \mathrm{l}$ (final 1x) ABsolute Blue SYBR Green Fluorescein Mix, $1 \mu \mathrm{l}$ cDNA (10-fold dilution) and $1 \mu \mathrm{l}(10 \mathrm{pmol})$ of each forward and reverse gene specific primers. PCR was run using CFX384 real-time PCR detection system (Bio-Rad laboratories, Inc) in 384 well plates with three technical replicates. The amplification conditions for all genes were: $95^{\circ} \mathrm{C}$ for $15 \mathrm{~min}$ to activate Thermo-Start ${ }^{\mathrm{TM}}$ DNA polymerase; followed by 35 cycles of denaturation $\left(95^{\circ} \mathrm{C}\right.$ for $\left.1 \mathrm{~min}\right)$, annealing $\left(64^{\circ} \mathrm{C} 30 \mathrm{~s}\right)$ and extension $\left(72^{\circ} \mathrm{C}\right.$ for $2 \mathrm{~min}$ ) with a final elongation step of $10 \mathrm{~min}$ at $72^{\circ} \mathrm{C}$. To ensure amplification specificity, a melting curve analysis $\left(65^{\circ} \mathrm{C}\right.$ to $95^{\circ} \mathrm{C}$ with a heating rate of $0.5^{\circ} \mathrm{C}$ per $5 \mathrm{~s}$ and continuous fluorescence measurement) was performed. Furthermore, amplified products were resolved on $1 \%$ 
agarose gel and size of DR-gene fragments were verified by comparison to a known molecular weight marker (HyperLadder ${ }^{\mathrm{TM}}$ 100bp Plus, Bioline, Germany).

\section{Relative gene expression analysis}

The relative expression of DR-genes was determined according to the Pfaffl method (Pfaffl, 2001) using

the formula: $\mathrm{R}=\left[\left(\mathrm{E}_{\text {target }}\right)^{\Delta \mathrm{Ct} \text { target (control-drought stressed) }}\right] /\left[\left(\mathrm{E}_{\text {ref }}\right)^{\Delta \mathrm{Ct} \text { ref (control-drought stressed) }}\right]$, where $\mathrm{R}$ is the relative expression ratio, $E_{\text {target }}$ is the real-time PCR efficiency of target genes (DR-genes) transcript, $E_{\text {ref }}$ is the real-time PCR efficiency of a reference gene (BnActin) transcript, $\mathrm{Ct}$ is the threshold cycle at which relative fluorescence of samples increased above the background fluorescence, and $\Delta \mathrm{Ct}$ is the difference between $\mathrm{Ct}$ values of control (100\% FC) and treated samples (30\% FC). Amplification efficiencies of reference and target genes were determined by using dilutions of DNA template. Relative expression values obtained from three biological replicates were used for statistical analysis.

\subsubsection{Data analysis}

The whole experiment was repeated twice. Mean data obtained from three replications of two independent experiments were analyzed using SAS version 9.3 (SAS Institute, Inc., Cary, NC, USA). To determine main and interactive effects of genotype, disease and drought stress factors, analysis of variance was performed by the mixed model procedure (PROC MIXED). Means were separated using Tukey's test at $5 \%$ level of significance. To analyze the relationships between disease severity, physiological and agronomic parameters, Pearson's linear correlation was performed and correlation coefficients were calculated using the PROC CORR procedure. To determine the relative contribution of variables to the variation in dry matter yield and identify most important variables contributing to the variation in dry matter yield, a stepwise multiple linear regression analysis was performed using the PROC REG procedure. A principal component analysis (PCA) was performed using the Minitab version 14 (Minitab, Coventry, UK).

\subsection{Results}

\subsubsection{V. longisporum disease development under drought stress conditions}

Typical symptoms of $V$. longisporum infection in Brassica napus under controlled environmental conditions are leaf yellowing, stunted growth and senescence of leaves and branches. In the VLsusceptible cultivar Falcon, the initial symptoms of VL infection (yellowing and stunted growth) were clearly observed two weeks after inoculation. In addition to this, most infected leaves showed severe shriveling, deformed shape and curling from the edge (Fig. 4.2). In the resistant genotype SEM however, 
these symptoms were distinctly observed relatively late (at $21 \mathrm{DPI}$ ) and with lower intensity. VL infection also induced production of excessive side branches in both susceptible and resistant genotypes (See section 4.4.4.2).

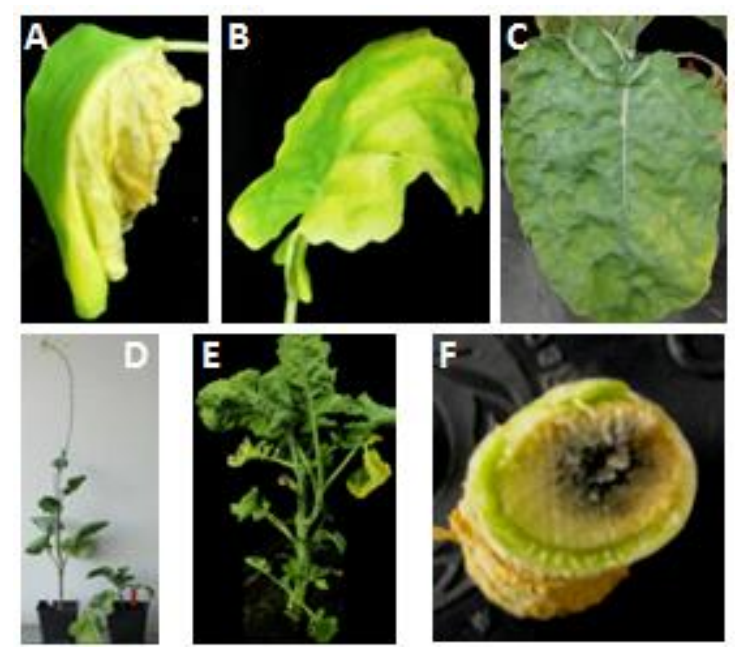

Figure $\quad 4.2$ Typical symptoms induced by Verticillium longisporum infection in Brassica napus under greenhouse condition. A, One sided chlorosis. B, Irregular shaped yellowing on leaf with dark veins. $\mathbf{C}$, Leaf shrivelling and deformation. D, Stunting: control (left); inoculated (right). E, Production of excessive abnormal branches and scabs on main stem showing the point of leaf senescence. F, Hypocotyl cross section showing vascular discoloration.

Disease development as measured by AUDPC was significantly affected by genotype and pathogen factors. The interaction of pathogen with genotype had also a significant effect on disease severity. However, drought alone and its interaction with genotype and pathogen had no effect (Table 4.9). Net AUDPC values generated from weekly disease index evaluation showed an increased rate of disease development over time. In cultivar Falcon, at 14 DPI first symptoms were observed and disease levels sharply increased towards $49 \mathrm{DPI}$. In genotype SEM however, not only that first symptoms were observed relatively late (zero net AUDPC at 14 DPI) but also there was only a very slight change in disease progress over time (Fig. 4.3). Furthermore, addition of drought stress, which begun at 21 DPI, caused no significant change in the response of this genotype to VL infection. At 28 DPI (one week after the beginning of drought stress treatments), similarly low disease levels (net AUDPC $=15$ ) were recorded from 100, 60 and 30\% FC treatments. Three weeks later (49 DPI), net AUDPC values of 100, 60 and 30\% FC were 36.2, 35.2 and 28.3, respectively, which was again not significantly different (Fig. 4.6). The uniform and significantly low levels of AUDPC values recorded at all watering conditions indicate stable resistance of genotype SEM under either sufficient or limited water supply conditions (Fig. 4.8).

Concerning the VL-susceptible genotype Falcon, significantly high disease levels were recorded at all watering regimes (Fig. 4.8; Fig. 4.6). Respective Net AUDPC values at 100, 60 and 30\% FC at 28 DPI were 35.7, 36.9 and 31.4. This is more than twice the values recorded in SEM. At 49 DPI, disease level was further increased to more than double resulting in respective net AUDPC values of 76.0, 83.5 and 71.1. 
The lowest net AUDPC values (in contrary to VL DNA) in both resistant and susceptible genotypes were recorded from 30\% FC treatments that represent extreme drought conditions (Fig. 4.6).

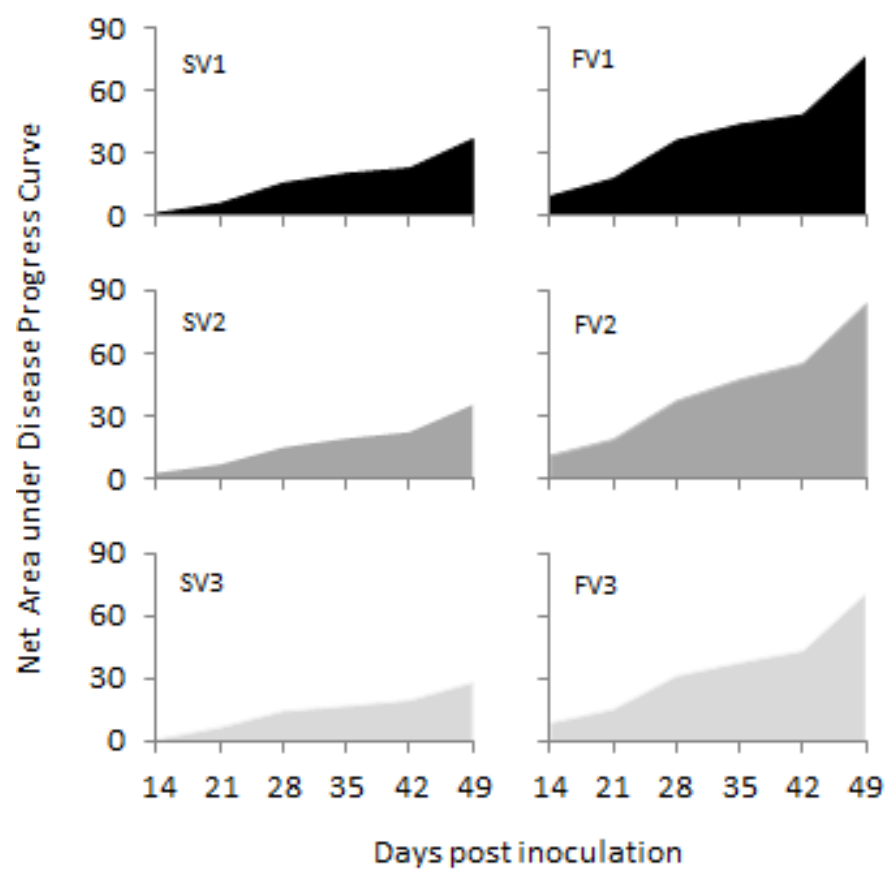

Figure 4.3 Net AUDPC of two Brassica napus genotypes infected with Verticillium longisporum under optimum watering (100\% FC) and different levels of drought stress conditions. AUDPC values are generated from weekly disease index measurements taken from 7 - 49 days post inoculation. SV, Tolerant genotype SEM-05-500526 VLinoculated. FV, Susceptible cultivar Falcon VL-inoculated. Treatment names followed by the numbers $\mathbf{1 ,} 2$ and 3 refers to watering at 100, 60 and $30 \%$ field capacity, respectively.

Molecular quantification of VL biomass by quantitative PCR also revealed the significant effect of genotype in disease development. The drought factor alone and its interaction with either genotype or disease had no significant effect (Table 4.9). Compared to Falcon, significantly low VL DNA was detected in SEM during early (28 DPI) as well as late (49 DPI) time points. At $28 \mathrm{DPI}$, fungal biomass in fully irrigated SEM plants was 15-fold lower than that of Falcon. This difference was even bigger (24-fold) when plants were exposed to severe drought. Quantification of VL DNA at 49 DPI also revealed that disease development in the resistant genotype SEM was not significantly altered due to the occurrence of drought. At this time point, the respective amount of VL DNA in this genotype at 100, 60 and 30\% FC was $27.1,29.0$ and $36.0 \mathrm{ng} / \mathrm{g}$ dry hypocotyl tissue. Nevertheless, this data still showed that the amount of fungal biomass in plant tissue increased as plants get exposed to drought stress (though the difference is very small and statistically insignificant). The situation was more aggravated in the susceptible cultivar Falcon where significantly evevated quantities of VL DNA were detected from severely stressed plants. When we compare fungal DNA at 28 and $49 \mathrm{DPI}$, at the later time point, quantity of VL DNA either remained constant or was slightly reduced in SEM while it significantly increased (up to 31-fold) in Falcon (Fig. 4.6). 
Disease development under different water supply conditions was further studied by assessment of stunting effects. Weekly measurement of plant height from 14 to 49 DPI showed that genotype, VL infection and their interaction have a significant effect on this parameter (Table 4.9). The effect of drought stress on plant height is discussed later in section 4.4.4.1. Regarding the effect of VL, even though infection significantly reduced plant height of both genotypes, the effect was by far stronger in Falcon than in SEM. Furthermore, the stunting effect of VL was more pronounced during early growth stage (28 DPI) compared to later growth periods (49 DPI). As mentioned earlier, in SEM plant height was not affected by VL infection until 14 DPI (Fig. 4.4) and only minor stunting was initially recorded at 21 DPI (data not shown). Significant and severe stunting effect on SEM was first observed at 28 DPI when $50 \%$ plant height reduction was recorded at $100 \%$ FC. Towards crop maturity again, plants were able to recover and relative stunting at $49 \mathrm{DPI}$ was reduced to $21.4 \%$. Interestingly, the stunting effect of VL under drought stress condition was significantly lower. At $28 \mathrm{DPI}$, stunting at $60 \%$ and $30 \% \mathrm{FC}$ were $31.2 \%$ and $37.9 \%$, respectively, which is by far lower than the effect at $100 \%$ FC. Further, at 49 DPI, mock and VL-inoculated plants had similar plant height both at moderate and severe drought stress conditions indicating that VL-infection has no stunting effect during drought stress in genotype SEM (Fig. 4.5; Table 4.7). For Falcon, it was the worst case where severe stunting already occurring at 14 DPI (Fig. 4.4) causing more than $70 \%$ reduction of plant height at 28 DPI under all watering conditions. Unlike in SEM, infected Falcon plants were not able to recover during later time points and as a result similarly strong stunting was observed at 49 DPI (Fig. 4.5; Fig. 4.8).
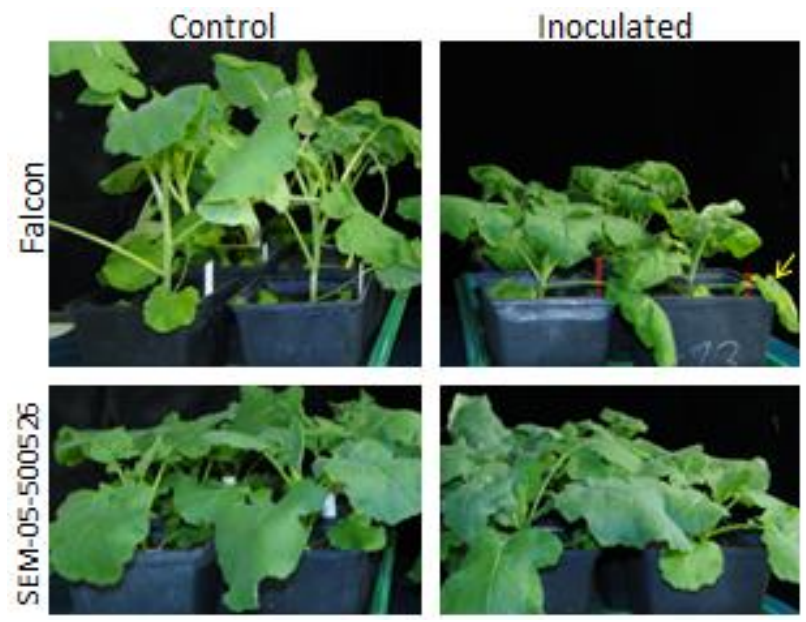

Figure 4.4 Phenotypes of susceptible (Falcon) and resistant (SEM-05-500526) Brassica napus plants two weeks after inoculation with Verticillium longisporum under greenhouse conditions. Vernalized seedlings were inoculated at $\mathrm{BBCH} 14$. At this time point, leaf yellowing (arrow) and stunting symptoms were only observed in Falcon. SEM plants showed no symptoms.

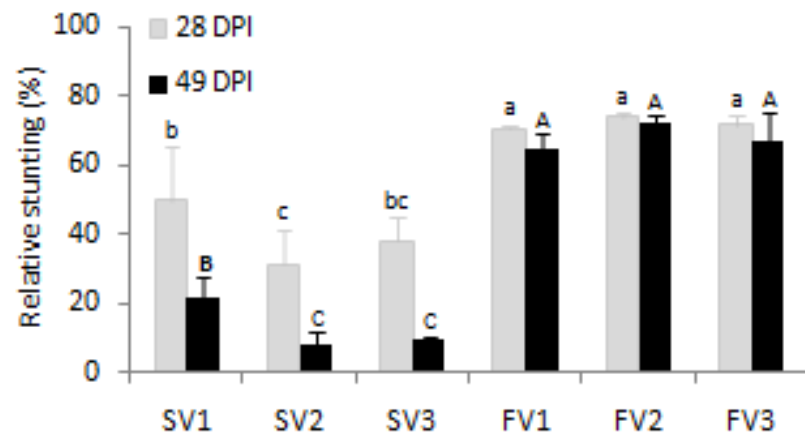

Figure 4.5 Stunting effect of Verticillium longisporum in Brassica napus under optimum watering and drought stress conditions. Relative stunting refers to percent plant height reduction in inoculated plants relative to the plant height of mock-inoculated plants. Mean data obtained from 30 plants of two independent experiments are presented. Bars indicate standard deviations. Different letters on the bars with the same letter-case indicate significant differences between treatments at $P \leq 0.05$. SV, Tolerant genotype SEM-05-500526 VL-inoculated. FV, Susceptible cultivar Falcon VL-inoculated. Treatment names followed by the numbers $\mathbf{1}, \mathbf{2}$ and $\mathbf{3}$ refer to watering at 100, 60 and $30 \%$ field capacity, respectively. 
The consistent response of susceptible and resistant $B$. napus genotypes to infection with VL under optimum watering and drought stress conditions was proven by Pearson correlation analysis that showed strong positive relationships between net AUDPC and relative stunting ( $r=0.95)$, net AUDPC and VL DNA ( $r=0.89)$ and relative stunting and VL DNA ( $r=0.95)$ measurements (Table 4.11). This observation was further supported by stepwise regression analysis that showed a significant contribution of the pathogen $\left(r^{2}=0.74\right)$ and genotype $\left(r^{2}=0.11\right)$ factors in determining disease development but not from drought stress (Table 4.12).

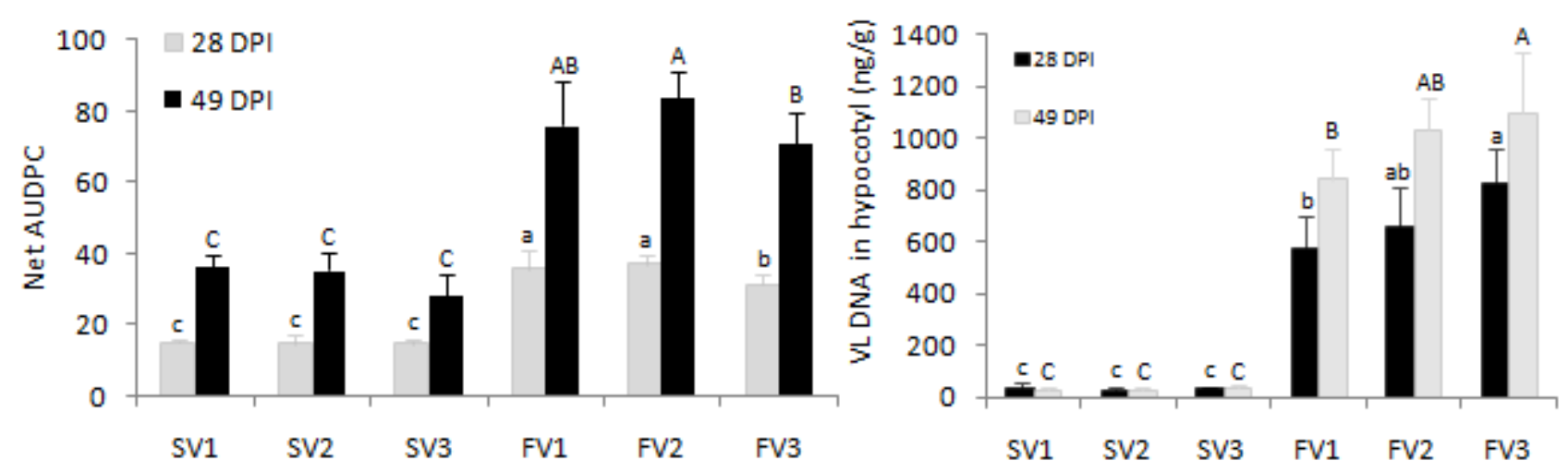

Figure 4.6 Effect of Verticillium longisporum in Brassica napus under optimum and drought stress conditions as measured by net AUDPC (Left) and quantification of VL DNA from dry hypocotyl tissue by quantitative PCR (right). Mean data obtained from 30 plants of two independent experiments are presented. Bars indicate standard deviations. For each parameter, different letters on the bars with the same letter-case indicate significant differences between treatments at $\mathrm{P} \leq 0.05$. SV, SEM-05-500526 VL-inoculated. FV, Falcon VL-inoculated. Treatment names followed by the numbers 1, 2 and $\mathbf{3}$ refer to watering at 100, 60 and 30\% field capacity, respectively.

\subsubsection{Physiological responses to $V$. longisporum infection and drought stress}

In order to investigate solitary and combined effects of drought stress and disease factors on plant physiology, several parameters that best explain the response of $B$. napus to water deficit were monitored at 28 and $49 \mathrm{DPI}$. These include gas exchange measurement, relative water content determination, proline content and gene expression analyses.

\subsubsection{Gas exchange}

Regardless of plant growth stage, the leaf gas exchange parameters [transpiration rate (E), stomatal conductance of $\mathrm{CO}_{2}$ (gs) and photosynthesis rate (A)] were only significantly affected by drought. Genotype and VL either independently, by interacting with each other, or by interacting with drought did not affect gas exchange (Table 4.9). As a result, no significant difference between genotypes and between mock- and VL-inoculated treatments was observed (Fig. 4.7). For genotype SEM, E at 100\% FC in mock-inoculated plants was $1.5 \mathrm{mmol} \mathrm{m}^{-2} \mathrm{~s}^{-2}$. A bit lower $\left(1.3 \mathrm{mmol} \mathrm{m}^{-2} \mathrm{~s}^{-2}\right)$ but insignificantly reduced E was recorded in VL-inoculated and similarly watered plants. Under severe stress conditions however, E 
was significantly reduced to 0.3 and $0.4 \mathrm{mmol} \mathrm{m}^{-2} \mathrm{~s}^{-2}$ in mock- and VL-inoculated treatments, respectively. For cultivar Falcon, more or less the same rates of leaf transpiration were recorded. In this cultivar, severe drought reduced E from 1.8 to 0.4 in the absence of VL infection and from 1.6 to 0.5 $\mathrm{mmol} \mathrm{m} \mathrm{m}^{-2} \mathrm{~s}^{-2}$ during infection with $\mathrm{VL}$. Regarding stomatal conductance of $\mathrm{CO}_{2}$, a similar trend was observed. In SEM, regardless of VL infection, watering at $30 \% \mathrm{FC}$ reduced gs from 0.13 to $0.02 \mathrm{~mol} \mathrm{~m}^{-2} \mathrm{~s}^{-2}$. In cultivar Falcon, gs was reduced from $0.1 \mathrm{~mol} \mathrm{~m}^{-2} \mathrm{~s}^{-2}$ (at 100\% FC) to 0.02 and $0.04 \mathrm{~mol} \mathrm{~m}^{-2} \mathrm{~s}^{-2}$ in mock and VL-inoculated 30\% FC treatments, respectively. In the same way, regardless of genotype and VLinfection, the photosynthesis rate was significantly reduced by severe drought stress. In SEM, a similar photosynthesis rate $\left(9.3 \mu \mathrm{mol} \mathrm{m} \mathrm{m}^{-2} \mathrm{~s}^{-1}\right)$ was measured from fully watered mock and VL-inoculated treatments. Watering at $30 \% \mathrm{FC}$ reduced $\mathrm{A}$ to 3.3 and $4.2 \mu \mathrm{mol} \mathrm{m} \mathrm{m}^{-1}$ in mock and VL-inoculated treatments, respectively. For cultivar Falcon, the mean photosynthesis rate at $100 \% \mathrm{FC}$ regardless of VL infection was $10 \mu \mathrm{mol} \mathrm{m} \mathrm{s}^{-1}$. Here, watering at 30\% FC again significantly reduced the rate to 2.7 and 4.4 $\mu \mathrm{mol} \mathrm{m} \mathrm{m}^{-1}$ under mock and VL- infection conditions, respectively (Fig. 4.7).

Although the analysis of all gas exchange measurements showed that VL infection has no significant effect, the rate by which drought induced gas exchange reduction in mock and VL-inoculated plants varied considerably. Compared to mock-inoculation, the rate of reduction in gas exchange due to drought stress was significantly lower during VL-infection (Table 4.5). For instance, the difference in photosynthesis rate between 100 and 30\% FC watered SEM plants in the absence of infection was 6.0 $\mu \mathrm{mol} \mathrm{m} \mathrm{s}^{-1}$. When the pathogen was present, this change was reduced to $5.1 \mu \mathrm{mol} \mathrm{m} \mathrm{s}^{-1}$. In a similar manner, this effect occurred in the VL-susceptible cultivar and was 7.1 and $5.7 \mu \mathrm{mol} \mathrm{m}^{-2} \mathrm{~s}^{-1}$ in control and infected treatments, respectively. Considering the transpiration rate, the respective reduction at $30 \% \mathrm{FC}$ in mock- and VL-infected plants was 3.8 and 2.3-fold in SEM and 4.0 and 2.1-fold in Falcon. Similar but relatively large differences were observed in stomatal conductance of $\mathrm{CO}_{2}$ where $30 \% \mathrm{FC}$ watering caused a reduction of 5.8 and 2.9-fold (in SEM) and 5.3 and 2.1-fold (in Falcon) in mock and VLinoculated treatments, respectively. The above observations suggest that biochemical or histological changes that might be induced by VL infection might help plants to partly mitigate drought stress. A positive role of VL-infection in reducing the impact drought stress under mild stress conditions (60\% FC) was not conclusive. Except for the slight reduction in VL-infected susceptible plants, gas exchange measurements at $28 \mathrm{DPI}$ also showed a similar trend (Appendix 4.1; Appendix 4.7). 


\subsubsection{Water use efficiency}

Instantaneous water use efficiency (WUE) computed as the ratio of photosynthesis to transpiration rate (Tambuss et al., 2007) is a key indicator of plant economic utilization of soil water. As expected, drought stress generally increased (irrespective of VL-infection) water use efficiency of both genotypes
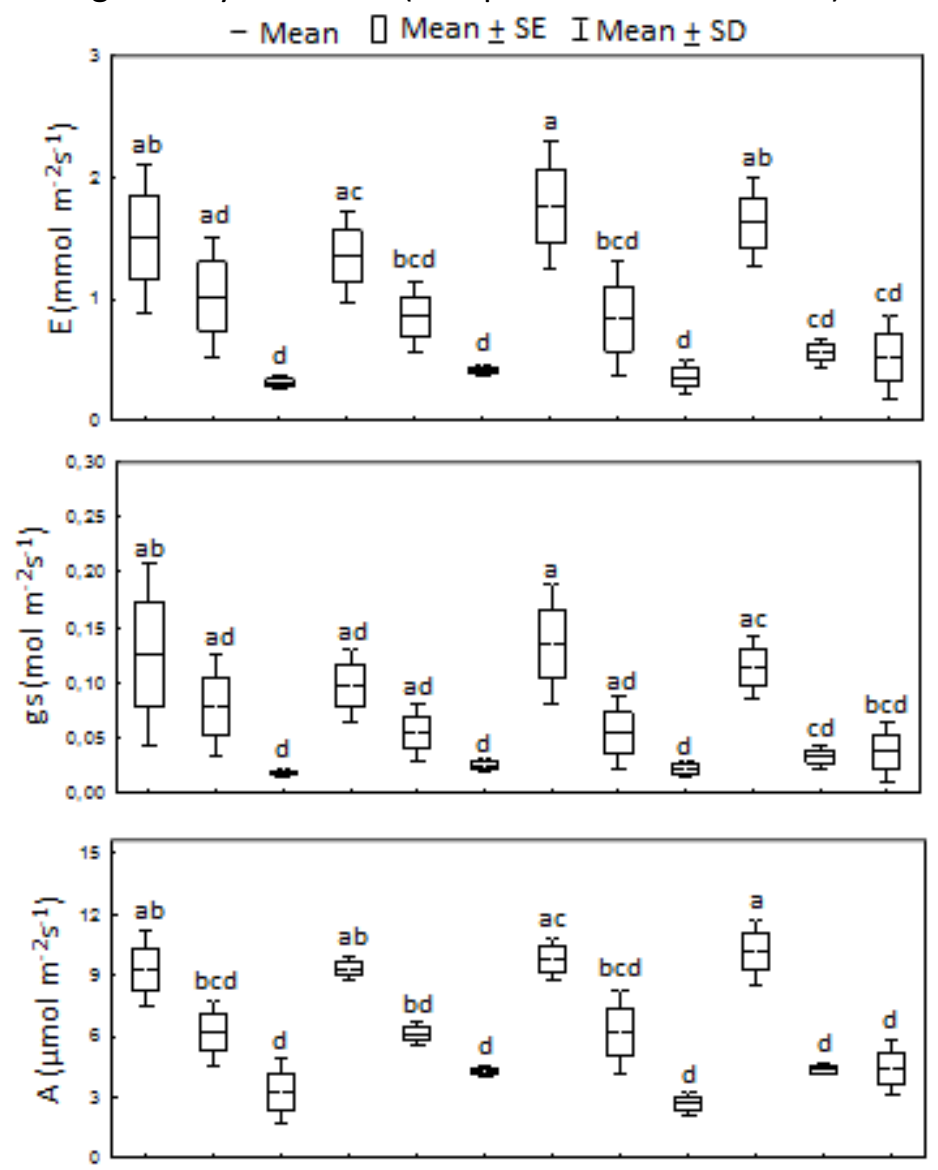

Figure 4.7 Transpiration rate $(\mathrm{E})$, stomatal conductance of $\mathrm{CO}_{2}$ (gs), photosynthesis rate (A), and water use efficiency (WUE) of two Brassica napus genotypes subjected to Verticillium longisporum infection and/or drought stress under greenhouse conditions. Mock and/or VLinoculated plants supplied with water at $100 \%$ FC were used as control. Drought treatments were made by exposing plants to moderate $(60 \% \mathrm{FC})$ or severe $(30 \% \mathrm{FC})$ drought stress for four weeks starting from 21 days after inoculation with Verticillium longisporum. Mean data obtained from 30 plants of two independent experiments are presented. For each parameter, different letters on the bars indicate significant differences between treatments at $P \leq 0.05$. SM, SEM05-500526 mock-inoculated. FM, Falcon mock-inoculated. SV, SEM-05-500526 VLinoculated. FV, Falcon VL-inoculated. Treatment names followed by the numbers 1, 2 and $\mathbf{3}$ refer to watering at 100,60 and $30 \%$ field capacity, respectively.

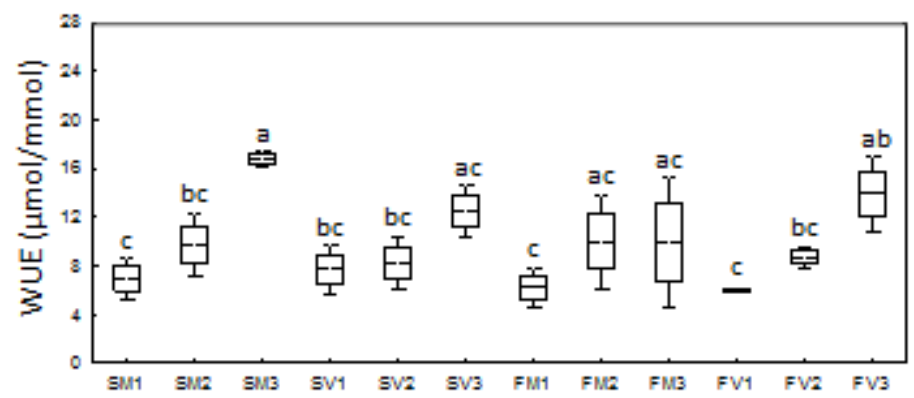

but significant increments were observed only at 30\% FC conditions (Fig. 4.7). In mock-inoculated treatments, due to the shift in water supply from $100 \%$ FC to $30 \%$ FC, WUE was significantly increased from 6.9 to $16.7 \mu \mathrm{mol} \mathrm{CO} \mathrm{mmol} \mathrm{H}_{2} \mathrm{O}^{-1}$ in SEM. In Falcon, it was increased from 6.2 to $9.9 \mu \mathrm{mol} \mathrm{CO}_{2} \mathrm{mmol}$ $\mathrm{H}_{2} \mathrm{O}^{-1}$ which is a slight change but still insignificant. The addition of the pathogen factor during drought stress resulted in slightly decreased WUE in SEM. For this genotype, compared to control conditions where $16.7 \mu \mathrm{mol}$ of $\mathrm{CO}_{2}$ was fixed per $1 \mathrm{mmol} \mathrm{H}_{2} \mathrm{O}$ transpired, during infection with VL, slightly less $\mathrm{CO}_{2}$ 
(12.5 $\mu \mathrm{mol})$ was fixed with the same amount of water transpired. Quite the opposite was observed in VL-susceptible cultivar Falcon. Here, WUE at $30 \% \mathrm{FC}$ in control plants was $9.9 \mu \mathrm{mol} \mathrm{CO}_{2} \mathrm{mmol} \mathrm{H}_{2} \mathrm{O}^{-1}$. During VL-infection, the efficiency was slightly increased to $13.9 \mu \mathrm{mol} \mathrm{CO}_{2} \mathrm{mmol} \mathrm{H}_{2} \mathrm{O}^{-1}$ (Table 4.5). Furthermore, although not statistically significant, a genotypic difference in WUE at 30\%FC was noticed in the absence of the pathogen as SEM had relatively higher WUE $\left(16.7 \mu \mathrm{mol} \mathrm{CO} \mathrm{Cmol} \mathrm{H}_{2} \mathrm{O}^{-1}\right)$ compared to Falcon $\left(9.9 \mu \mathrm{mol} \mathrm{CO} \mathrm{Cmol} \mathrm{H}_{2} \mathrm{O}^{-1}\right.$ ). During VL-infection, both genotypes had similar WUE (Fig. 4.7). A similar trend was observed from the data measured a week after initiation of drought treatments, 28 DPI (Appendix 4.1; Appendix 4.7).

Single and interactive effects of drought and V. longisporum in gas exchange of VL-susceptible and VLresistant $B$. napus genotypes were further analysed by stepwise multiple regression analysis. The results showed that gas exchange was only significantly affected by drought stress and this stress factor contributed up to $77 \%$ of the variation in gas exchange. On the other hand, genotype and disease factors alone or all their possible interactions had no significant effect (Table 4.14). The strong correlations (up to $98 \%$ ) among gas exchange parameters and between gs exchange and other physiological parameters (Table 4.11) support the finding that major changes in physiological parameters is mainly due to drought stress.
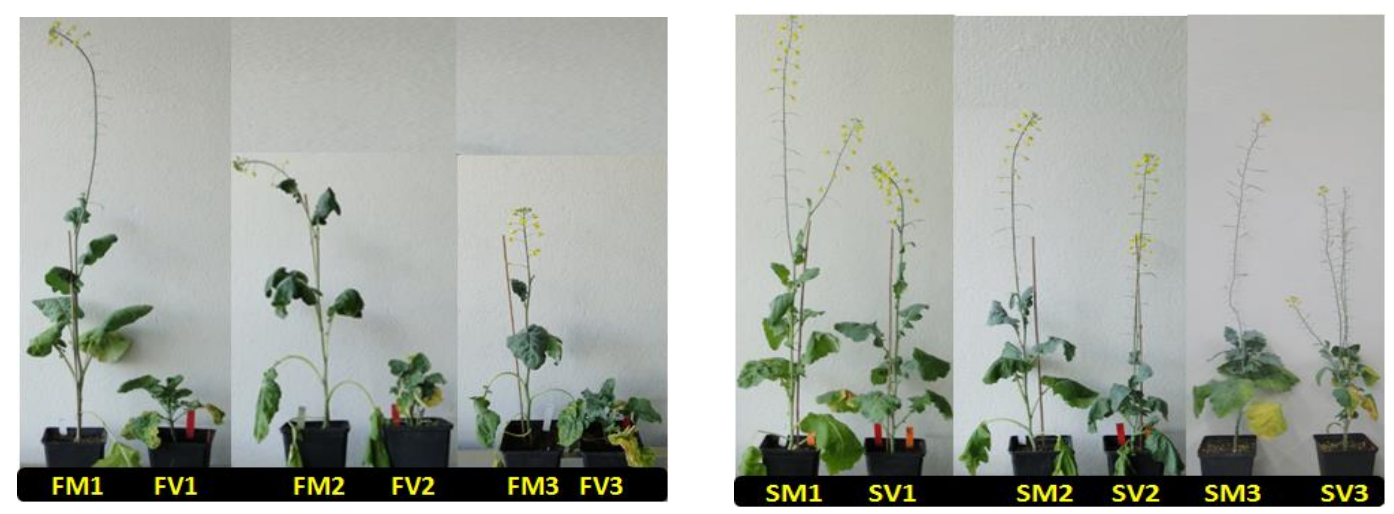

Figure 4.8 Phenotypes of randomly selected Brassica napus plants exposed to drought stress and/or Verticillium longisporum infection in a greenhouse experiment 28 days after initiation of drought treatments (49 DPI). Vernalized seedlings were inoculated at $\mathrm{BBCH} 14$ by submerging injured roots in spore suspension (10 $\left.{ }^{6} \mathrm{conidia} / \mathrm{ml}\right)$ for 30 minutes. Mock-inoculated plants were similarly dipped in autoclaved tap water. SM, SEM-05-500526 mockinoculated. FM, Falcon mock-inoculated. SV, SEM-05-500526 VL-inoculated. FV, Falcon VL-inoculated. Treatment names followed by the numbers 1, 2 and 3 refer to watering at 100, 60 and 30\% field capacity, respectively.

\subsubsection{Leaf relative water content}

RWC represents the actual water status of pant tissue and provides the most meaningful index to evaluate tolerance of plants to drought stress. In the present study, measurement of this parameter was also done one (28 DPI) and four weeks (49 DPI) after initiation of drought treatments. According to the 
results, leaf RWC was only affected by severe drought stress. Besides, the effect of drought on RWC was dependent on plant genotype and growth stage. One week after initiation of the drought treatments (28 DPI; $\mathrm{BBCH}$ 50-60; inflorescence emergence stage), for most treatments, the plant water status was not significantly affected by drought. At this time point, except for VL-infected Falcon plants, a slight and insignificant reduction ranging from 6 to $7 \%$ was recorded at $60 \%$ and $30 \%$ FC watering treatments of both genotypes. At flowering growth stage (BBCH 60-70; $49 \mathrm{DPI}), \mathrm{RWC}$ due to severe drought was only significantly reduced in genotype SEM where RWC was reduced by 13 and $14 \%$ in mock and VL-infected treatments, respectively. The respective values for genotype Falcon were 10.3 and $10.1 \%$ (Table 4.4).

Table 4.4 Effect of different levels of drought stress and Verticillium longisporum infection on leaf relative water content (\%) of two Brassica napus genotypes 7 and 28 days after initiation of drought treatments, at 28 and $49 \mathrm{DPI}$, respectively.

\begin{tabular}{cccc|ccc}
\hline Treatments & \multicolumn{3}{c|}{$28 \mathrm{DPI}$} & \multicolumn{3}{c}{$49 \mathrm{DPI}$} \\
& $100 \% \mathrm{FC}$ & $60 \% \mathrm{FC}$ & $30 \% \mathrm{FC}$ & $100 \% \mathrm{FC}$ & $60 \% \mathrm{FC}$ & $30 \% \mathrm{FC}$ \\
\hline SM & $91 \pm 2^{\mathrm{a}}$ & $83 \pm 4^{\mathrm{ab}}$ & $84 \pm 2^{\mathrm{ab}}$ & $87 \pm 2^{\mathrm{abc}}$ & $83 \pm 1^{\mathrm{ad}}$ & $74 \pm 4^{\mathrm{d}}$ \\
SVL & $90 \pm 4^{\mathrm{a}}$ & $85 \pm 4^{\mathrm{ab}}$ & $84 \pm 1^{\mathrm{ab}}$ & $91 \pm 4^{\mathrm{ab}}$ & $85 \pm 6^{\mathrm{ad}}$ & $77 \pm 5^{\mathrm{cd}}$ \\
FM & $92 \pm 2^{\mathrm{a}}$ & $87 \pm 3^{\mathrm{ab}}$ & $86 \pm 1^{\mathrm{ab}}$ & $91 \pm 2^{\mathrm{a}}$ & $86 \pm 4^{\mathrm{abc}}$ & $81 \pm 6^{\mathrm{ad}}$ \\
FVL & $92 \pm 4^{\mathrm{a}}$ & $84 \pm 4^{\mathrm{a}}$ & $81 \pm 3^{\mathrm{b}}$ & $89 \pm 2^{\mathrm{ab}}$ & $86 \pm 1^{\mathrm{abc}}$ & $79 \pm 4^{\mathrm{bd}}$ \\
\hline
\end{tabular}

Drought treatments were made by exposing plants to moderate $(60 \% \mathrm{FC})$ or severe $(30 \% \mathrm{FC})$ drought stress for four weeks starting from 21 days after inoculation with Verticillium longisporum. Mock and/or VL-inoculated plants supplied with water at $100 \%$ FC were used as control. Data shown is mean values \pm standard deviations obtained from 30 plants of two independent experiments. Mean values containing the same superscript are not significant at $\mathrm{P}=0.05$. FC, Field capacity. SM, SEM-05-500526 mock- inoculated. FM, Falcon mock-inoculated. SVL, SEM-05500526 VL- inoculated. FVL, Falcon VL-inoculated.

\subsubsection{Proline content}

Another drought stress response indicator exploited in this study was proline content, a universal biomarker for stress analysis in plants (Ernst and Peterson, 1994). Proline was extracted from leaf and hypocotyl tissue and concentrations were quantified by referring to the standard curve produced from known concentrations of proline (Fig. 4.9). The results showed significant effects of all experimental factors (Table 4.10) and a wide range of variation ( 3 to 19 fold) in proline levels in response to drought stress or VL infection, and dependent on the plant part and also to some extent to the plant genotype. The amount of proline also increased with increasing severity of drought stress (Fig. 4.10). To begin with the genotypic differences, the two genotypes not only showed differences in the initial free proline content, but also in the quantity of drought induced proline accumulation. Although this was not statistically significant, the initial proline content seems to be higher in Falcon than in SEM. However, 
the drought induced proline accumulation was significantly higher in SEM than in Falcon (Fig. 4.10; Table 4.5).

Drought stress was the most crucial factor that caused pronounced accumulation of free proline in leaf and hypocotyl tissues. Under normal growth conditions where there was no stress from disease or drought, the proline content in SEM plants was $3.9 \mu$ moles/g in leaf and $2.2 \mu$ moles/g in hypocotyl tissues. At $30 \%$ FC, these values increased 12 fold in leaf and 19 fold in hypocotyl tissue. In cultivar Falcon, the proline content in control treatments was $6.9 \mu$ moles/g in leaves and $1.5 \mu$ moles/g in hypocotyls. Similarly, drought stress at 30\% FC induced an increase of 4 and 11fold in leaf and hypocotyl tissue, respectively. As in non-infected control treatments, a significant increase in free proline accumulation due to drought stress was observed in VL-inoculated treatments. Furthermore, the comparison of drought induced proline synthesis in mock- and VL-inoculated treatments showed that VL infection caused a significant reduction in proline synthesis in genotype SEM but had no such impact in Falcon (Fig. 4.10).
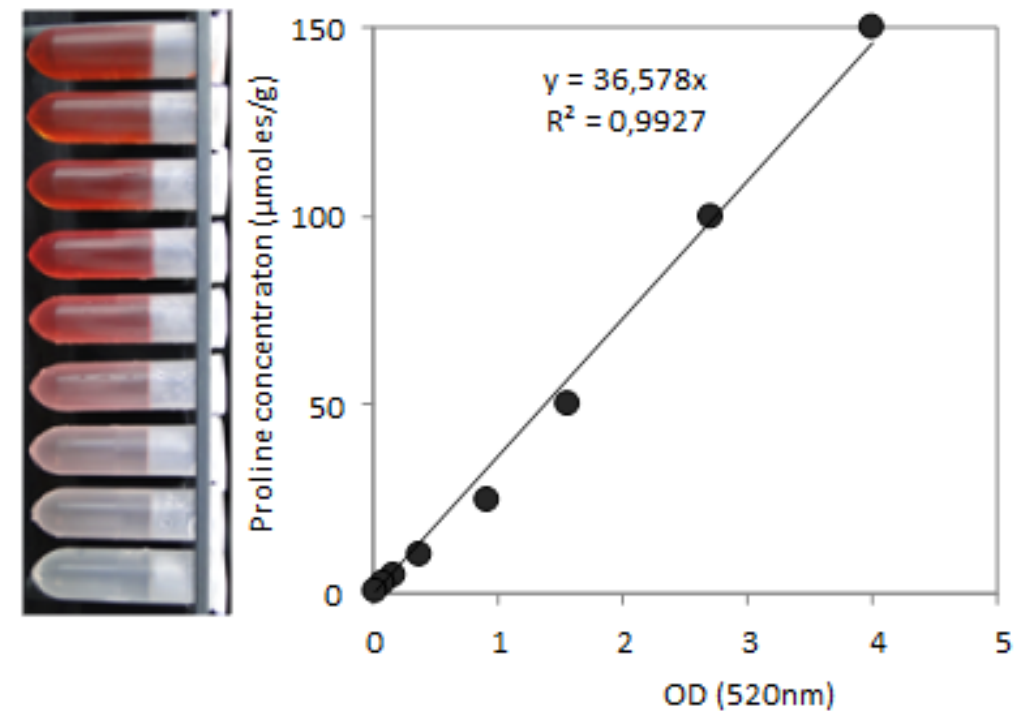

Figure 4.9 Standard curve for quantification of proline concentration in leaf and hypocotyl plant tissues of $B$. napus. Data points represent means of triplicates. Known concentrations of L-proline ranging between 150 and 0.5 $\mu \mathrm{g} / \mathrm{ml}$ were prepared in $3 \%$ aqueous sulfosalicylic acid.

The forth important factor that showed a difference in proline contents was the plant part. Generally, the initial proline content was significantly higher in leaves than in hypocotyl tissue (Fig. 4.10). However, the drought induced proline synthesis in the presence and absence of the pathogen showed contrasting pattern in different plant parts. On the one hand, drought (30\% FC) induced proline synthesis in mockinoculated plants was significantly higher in hypocotyl than in leaf tissue. On the other hand, during VLinfection, increase in proline content due to watering at 30\% FC became higher in leaf tissue compared to the hypocotyl, meaning that upon infection with $V L$, drought induced proline synthesis increased in leaves while it decreased in the hypocotyl (Table 4.5). As it was previously shown with other physiological parameters, the relatively high accumulation of proline in hypocotyl tissue during VL- 
infection might again indicate a reduced impact of drought stress due to VL infection. This is strongly supported by the close correlation between proline synthesis and the physiological parameters considered in this study (Table 4.11). Analysis of variance using the mixed model procedure also showed that unlike other physiological traits, all the three factors (Genotype, VL infection and drought stress) and their interaction significantly affected proline synthesis (Table 4.10) suggesting that proline may play a role in the B. napus-VL interaction under drought stress conditions. Analysis of proline content in leaf and hypocotyl tissue samples collected at $28 \mathrm{DPI}$, one week after initiation of drought treatments, showed a similar but even stronger impact of plant genotype and plant part (Appendix 4.2; Appendix 4.8).

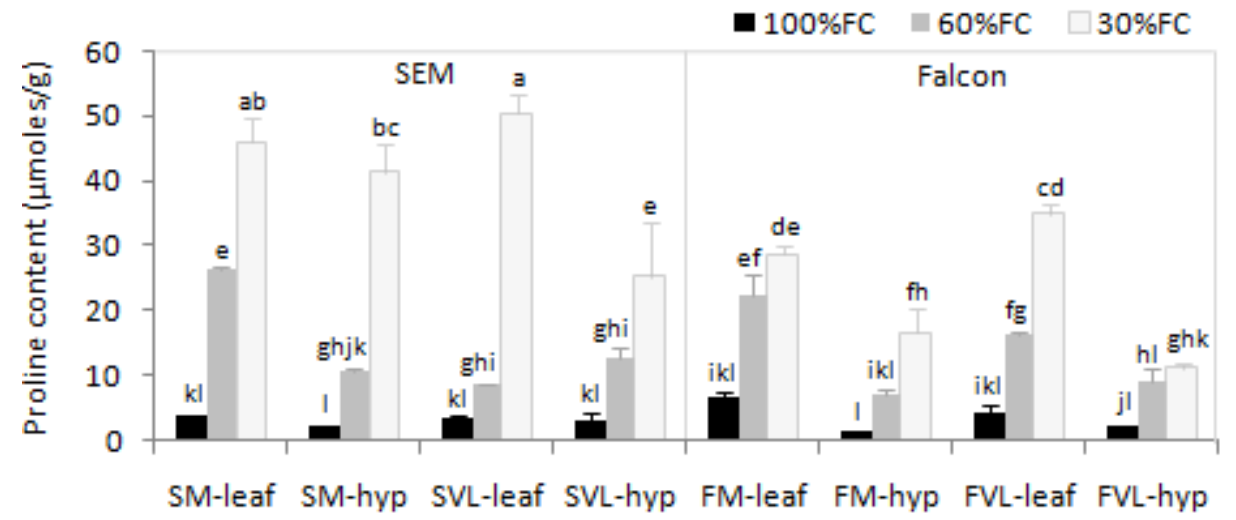

Figure 4.10 Changes in proline content in leaf and hypocotyl tissue of two Brassica napus genotypes exposed to different levels of drought stress, and Verticillium longisporum infection. Drought treatments were made by exposing plants to moderate $(60 \%$ FC) or severe (30\% FC) drought for four weeks starting from 21 days after inoculation with Verticillium longisporum. Mock and/or VL-inoculated plants supplied with water at $100 \%$ FC were used as control. Mean data obtained from 30 plants of two independent experiments are presented. Bars indicate standard deviations. Different letters on the bars indicate significant differences between treatments at $P \leq 0.05$. FC, Field capacity. hyp, Hypocotyl. SM, SEM-05-500526 mock- inoculated. FM, Falcon mock-inoculated. SVL, SEM05-500526 VL- inoculated. FVL, Falcon VL-inoculated.

Table 4.5 Comparison of physiological measurements in fully irrigated (100\% FC) and severely stressed (30\% FC) Brassica napus genotypes under control and V. longisporum infection conditions.

\begin{tabular}{|c|c|c|c|c|c|c|c|c|}
\hline \multirow{2}{*}{ Parameters } & \multicolumn{4}{|c|}{ Relative change } & \multicolumn{4}{|c|}{ Fold change } \\
\hline & SM & SVL & FM & FVL & SM & SVL & FM & FVL \\
\hline $\mathrm{E}\left(\mathrm{mmol} \mathrm{m} \mathrm{m}^{-2} \mathrm{~s}^{-1}\right)$ & -1.19 & -0.94 & -1.41 & -1.11 & -3.77 & -2.30 & -3.95 & -2.14 \\
\hline gs $\left(\mathrm{mol} \mathrm{m}^{-2} \mathrm{~s}^{-1}\right)$ & -0.11 & -0.07 & -0.11 & -0.08 & -5.80 & -2.94 & -5.34 & -2.05 \\
\hline A $\left(\mu \mathrm{mol} \mathrm{m} \mathrm{m}^{-2} \mathrm{~s}^{-1}\right)$ & -6.04 & -5.06 & -7.13 & -5.72 & -1.85 & -1.19 & -2.65 & -1.29 \\
\hline WUE $\left(\mu \mathrm{mol} \mathrm{CO} \mathrm{Cmmol} \mathrm{H}_{2} \mathrm{O}^{-1}\right)$ & +9.85 & +4.75 & +3.76 & +8.00 & +1.43 & +0.62 & +0.61 & +1.34 \\
\hline RWC (\%) & -13.0 & -14.0 & -10.3 & -10.1 & -0.08 & -0.18 & -0.13 & -0.13 \\
\hline LPC ( $\mu$ moles/g) & +42.1 & +46.8 & +21.9 & +30.5 & +10.70 & +12.80 & +3.20 & +6.70 \\
\hline HPC ( $\mu$ moles $/ \mathrm{g}$ ) & +39.2 & +22.1 & +15.3 & +9.0 & +17.60 & +6.60 & +10.40 & +3.80 \\
\hline
\end{tabular}

Relative change values were obtained by subtracting mean values of severely stressed (30\% FC) treatments from respective values of fully irrigated $(100 \% \mathrm{FC})$ treatments. Fold change was calculated using the formula 1 minus ratio of values at $100 \%$ FC to $30 \%$ FC treatments. Negative and positive signs indicate increase and decrease in physiological processes, respectively. Data measured 28 days after initiation of drought treatments (49 DPI) were used. SM, SEM-05-500526 mock- inoculated. FM, Falcon mock-inoculated. SVL, SEM-05-500526 VL- inoculated. FVL, Falcon VL-inoculated. E, transpiration rate. gs, stomatal conductance of $\mathrm{CO}_{2}$. A, photosynthetis rate. WUE, water use efficiency. RWC, leaf relative water content. LPC, leaf proline content. HPC, hypocotyl proline content. 


\subsubsection{Effect of drought stress and $V$. longisporum infection on expression of drought responsive genes}

The expression of drought inducible genes in mock- and VL- inoculated plants was analyzed by real time quantitative polymerase chain reaction (RT-qPCR). In this procedure, the transcript level of drought responsive genes in severely stressed plants ( $30 \% \mathrm{FC}$ ) was compared with the level in the respective fully irrigated (100\% FC) treatments. Before expression analysis, sequence identity of eight drought responsive (DR) genes was verified by sequencing of cloned PCR products (Table 4.6). Of these, RT-PCR amplification was not successful for the two late embryogenesis abundant (LEA) genes (BnLEA4-1 and ME-leaN4).

Table 4.6 Analysis of nucleotide identity of Brassica napus reference and drought inducible genes

\begin{tabular}{|c|c|c|c|c|c|}
\hline $\begin{array}{c}\text { Gene } \\
\text { symbol }\end{array}$ & Gene name & $\begin{array}{l}\text { Size } \\
\text { (bp) }\end{array}$ & $\begin{array}{l}\text { Genbank } \\
\text { accession }\end{array}$ & E-Value & $\begin{array}{c}\% \\
\text { identity }\end{array}$ \\
\hline BnActin & B. napus Actin & 262 & DQ370142.1 & $2 e-106$ & $98 \%$ \\
\hline$B n C B F 17$ & B. napus C-repeat binding factor & 199 & AF499034.1 & $2 \mathrm{e}-70$ & $94 \%$ \\
\hline CIPK1 & B. napus Calcineurin B-like (CBL)-interacting protein kinase & 314 & GU189585.1 & $6 e-35$ & $98 \%$ \\
\hline BnDREB2-23 & B. napus drought-responsive element binding factor & 553 & AY444874.1 & 0.0 & $99 \%$ \\
\hline BnHB6 & B. napus Homeodomain Leucine-Zipper & 840 & AF268422.1 & $2 \mathrm{e}-148$ & $97 \%$ \\
\hline$B n P 5 S C 1$ & B. napus delta 1-pyrroline-5-carboxylate synthetase A & 220 & AF314811.1 & $4 e-82$ & $98 \%$ \\
\hline BnP5SC2 & B. napus delta 1-pyrroline-5-carboxylate synthetase B & 200 & AF314812.1 & $1 e-69$ & $100 \%$ \\
\hline$B n L E A 4-1$ & Brassica napus group 4 late embryogenesis abundant gene & 479 & AY572958.1 & 0.0 & $97 \%$ \\
\hline ME-leaN4 & Brassica napus group 3 late embryogenesis abundant gene & 552 & AB083361.1 & 0.0 & $97 \%$ \\
\hline
\end{tabular}

PCR amplification was performed using high fidelity DNA polymerase ( $P f u$ DNA polymerase) with gene specific primers. PCR products were excised from the gel, cloned into a pBluescript SK vector and sequenced. Sequence verification was performed using the NCBI blastn engine.
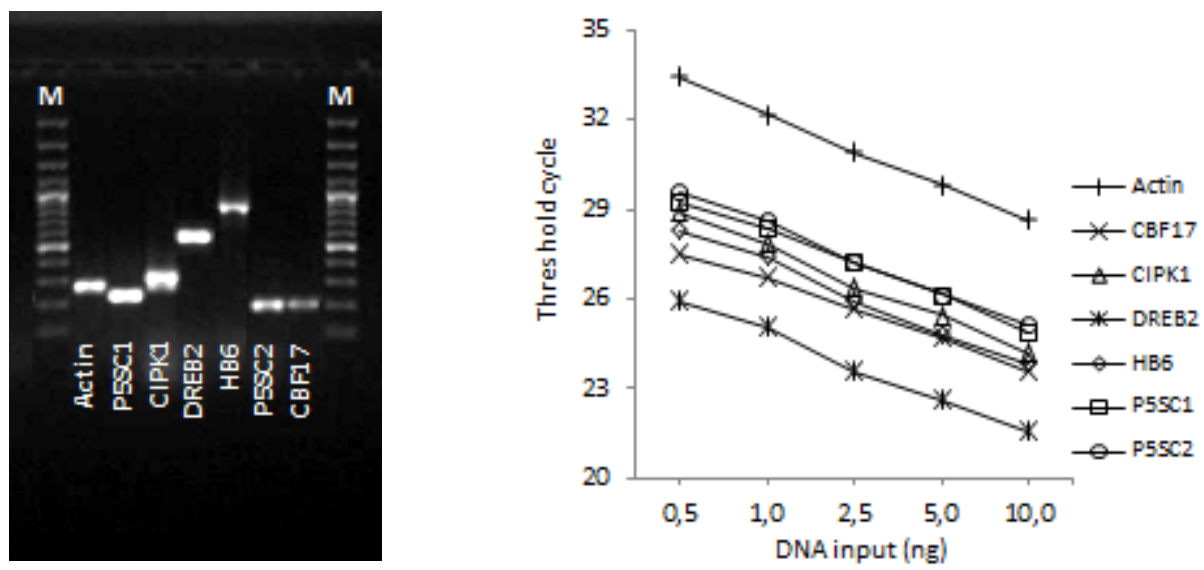

Figure 4.11 Analysis of specificity of RT-PCR products (left) and real time PCR efficiency (right) of reference and drought inducible genes in Brassica napus. M, 100bP molecular marker. Actin, reference gene $\left(R^{2}=0.988\right.$; slope 3.474). P5SC1, delta 1-pyrroline-5-carboxylate synthetase $A\left(R^{2}=0.994\right.$; slope -3.244$)$. CIPK1, Calcineurin $B$-like $(C B L)$-interacting protein kinase $\left(R^{2}=0.997\right.$; slope -3.550$)$. DREB2, Drought-responsive element binding factor $\left(R^{2}=\right.$ 0.999; slope -3.351). HB6, Homeodomain Leucine-Zipper $\left(R^{2}=0.998\right.$; slope -3.431). P5SC2, delta 1-pyrroline-5carboxylate synthetase $B\left(R^{2}=0.999\right.$; slope -3.428$)$. CBF17, C-repeat binding factor $\left(R^{2}=0.998\right.$; slope -3.320$)$. For gene expression analysis, RT-PCR efficiency of each gene was calculated using the formula $E=10^{\left(-1 / s^{\prime l o p e)}\right.}$ (Pfaffl, 2001). 

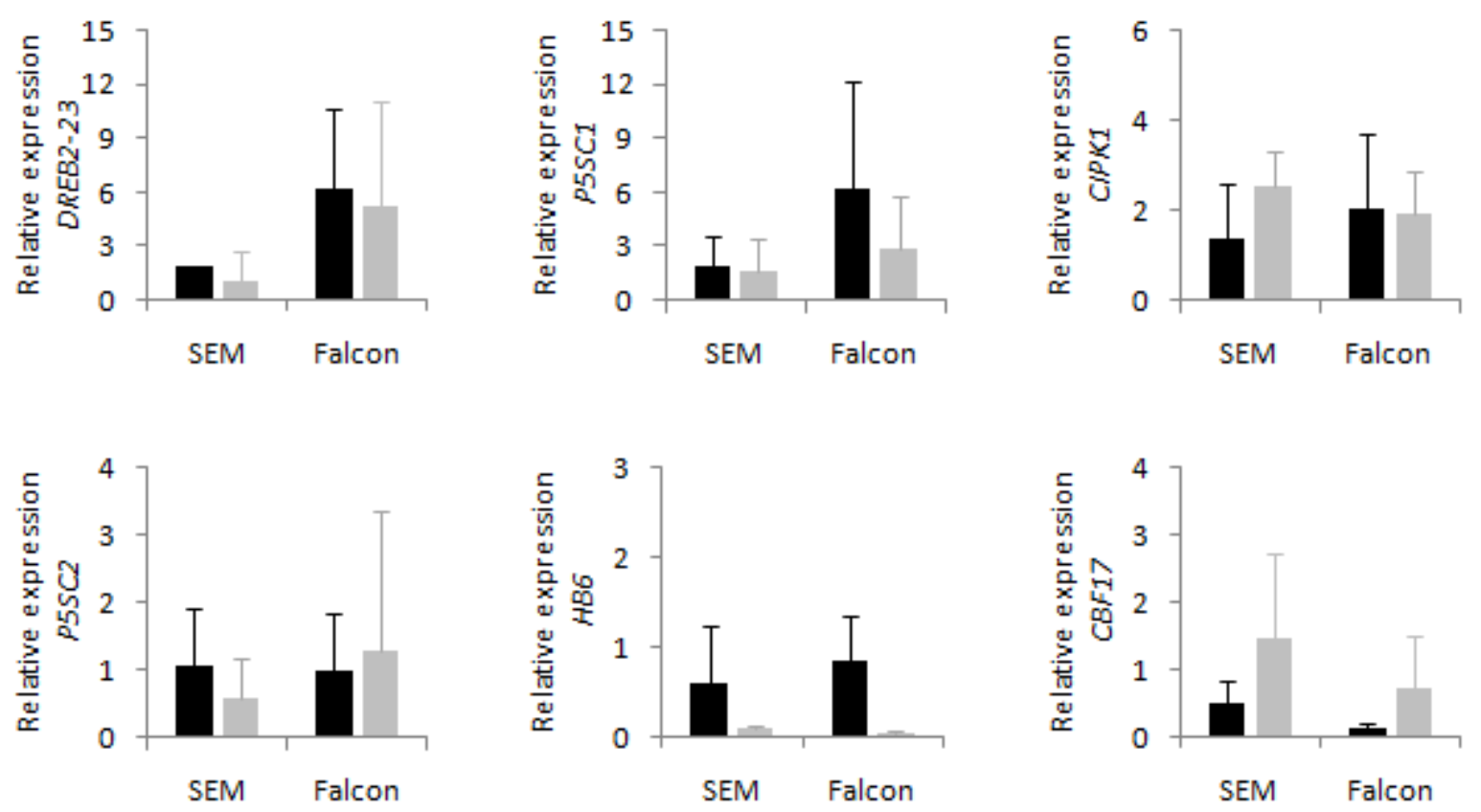

Figure 4.12 RT-PCR analysis of the expression of drought inducible genes in two Brassica napus genotypes subjected to drought stress and/or Verticillium longisporum infection. Total RNA was extracted from leaf samples collected 7 days after exposure to drought stress (28 days after inoculation with Verticillium longisporum). Data show the fold changes in gene expression in drought stressed (30\% field capacity) vs. optimally watered (100\% field capacity) mock (black bars) and Verticillium longisporum infected (grey bars) plants. Expression levels were normalized using BnActin gene according to Pfaffl, 2001. Error bars represent standard deviations of means obtained from three independent biological replicates each consisting of 10 plants. DREB2, Drought-responsive element binding factor. P5SC1, delta 1-pyrroline-5-carboxylate synthetase A. CIPK1, Calcineurin B-like (CBL)interacting protein kinase. P5SC2, delta 1-pyrroline-5-carboxylate synthetase B. HB6, Homeodomain LeucineZipper. CBF17, C-repeat binding factor.

Analysis of RT-PCR-products by gel electrophoresis showed the desired single product size of each gene, corroborating specificity of the RT-PCR (Fig. 4.11). Melting curve analysis also showed single product specific melting temperature of $84^{\circ} \mathrm{C}$ for actin, $84.5^{\circ} \mathrm{C}$ for CBF17, $82.0^{\circ} \mathrm{C}$ for CIPK1, $88.5^{\circ} \mathrm{C}$ for DREB2, $88.5^{\circ} \mathrm{C}$ for $\mathrm{HB} 6,82.5^{\circ} \mathrm{C}$ for $\mathrm{P} 5 \mathrm{SC} 1$ and $83.0^{\circ} \mathrm{C}$ for $\mathrm{P} 5 \mathrm{SC} 2$. Relative gene expression was calculated considering RT-PCR efficiency of each gene (Fig. 4.11) and normalized using the BnActin gene transcripts according to Pfaffl, 2001. Results showed that regardless of the plant genotype and VL-infection, up to 6 fold increase in gene expression was induced by the severe drought treatment. The P5SC1 gene that regulates proline biosynthesis and the transcription factors DREB2-23 and CIPK1 that control the expression of stress-responsive genes via ABA-independent pathway showed the highest level of expression. Compared to the aforementioned three genes however, the expression of P5SC2, CBF17, and HB6 was relatively lower, with about a one fold increase (Fig. 4.12). Mixed model analysis of variance showed no significant differences between genotypes and between mock and VL-inoculated treatments. Nevertheless, it was observed that the transcript level of P5SC1, DREB2 and CIPK1 genes were relatively higher in Falcon than in SEM. In both genotypes, VL-infection slightly increased 
expression of CBF17 while it decreased HB6. In cultivar Falcon, infection slightly reduced expression of P5SC1 and HB6. In SEM, DREB2, P5SC2 and HB6 were slightly reduced in infected treatments while CIPK1 and CBF17 were increasing.

\subsubsection{Effect of $V$. longisporum and drought stress on yield and morphological traits}

Due to a limited supply of water and mineral nutrients, plant growth and yield can considerably be reduced during drought periods. This condition may be aggravated if a biotic stress factor (e.g. disease) is added. In the present study, in order to assess single and interactive effects of genotype, drought, and $V$. longisporum infection on plant growth and to further estimate the magnitude of yield loss due to these factors, plant height, branch development, stem thickness and dry matter yield accumulation were assessed one and four weeks after the initiation of drought treatments, at 28 and 49 DPI, respectively.

\subsubsection{Plant height}

As expected, VL infection and exposure to drought stress significantly reduced plant height. Mixed model ANOVA showed that not only genotype, disease and drought independently, but also disease*genotype and disease*drought interactions significantly affected plant height (Table 4.9). This is also clearly seen in correlation analysis results that showed strong relationship of this parameter with disease, physiological and other agronomic traits (Table 4.11). Under normal growing conditions, the two genotypes showed comparable growth and attained similar plant height at crop maturity stage (49 DPI). However, when plants were exposed to drought or disease stress factors, they responded differently. The effect of VL-infection on plant growth in the susceptible cultivar Falcon was noticed two weeks after inoculation (Fig. 4.3) and a week later (21 DPI) for the resistant genotype SEM (data not shown). However, the effect of drought stress was only visible after two weeks of exposure to drought (35 DPI), the effect becoming evident and significant in plant height reduction in both genotypes (data not shown). Similar to early time points, four weeks after initiation of drought treatments (49 DPI), the stunting effects of $V L$ and drought were also dependent on the genotype (Table 4.7). In SEM, a reduction of plant height due to VL was only observed at full water supply ( $100 \% \mathrm{FC}$ ) but not at moderate (60\% FC) and severe (30\% FC) drought levels. Moreover, it was only under non-inoculated conditions that moderate and severe drought stresses caused significant reductions in plant height, possibly suggesting reduced stunting effect of drought in VL-infected treatments in this genotype. Contrary to SEM and irrespective of water supply, VL infection caused severe plant height reduction in Falcon. Interestingly, only severe drought stress caused stunted growth in mock-inoculated Falcon plants, suggesting less 
drought sensitivity of this genotype compared to SEM in which moderate stress also significantly reduced plant height. Due to the extremely severe stunting effect of VL which probably masked the effect of drought stress, the effect of drought on plant height in VL-inoculated Falcon plants was not possible to determine (Table 4.7).

Table 4.7 Plant height $(\mathrm{cm})$ of two Brassica napus genotypes exposed to drought stress and/or infection with Verticillium longisporum.

\begin{tabular}{ccccccc} 
& \multicolumn{3}{c}{$28 \mathrm{DPI}$} & & \multicolumn{3}{c}{$49 \mathrm{DPI}$} \\
Treatments & $100 \% \mathrm{FC}$ & $60 \% \mathrm{FC}$ & $30 \% \mathrm{FC}$ & $100 \% \mathrm{FC}$ & $60 \% \mathrm{FC}$ & $30 \% \mathrm{FC}$ \\
\hline SM & $74 \pm 20^{\mathrm{a}}$ & $50 \pm 11^{\text {ae }}$ & $51 \pm 12^{\text {ad }}$ & $140 \pm 6^{\mathrm{A}}$ & $107 \pm 11^{\mathrm{B}}$ & $104 \pm 7^{\mathrm{B}}$ \\
SVL & $37 \pm 11^{\text {cdef }}$ & $51 \pm 8^{\text {ad }}$ & $45 \pm 8^{\text {bcdef }}$ & $111 \pm 8^{\mathrm{B}}$ & $98 \pm 3^{\mathrm{B}}$ & $95 \pm 7^{\mathrm{B}}$ \\
FM & $70 \pm 9^{\text {ab }}$ & $63 \pm 5^{\text {ac }}$ & $59 \pm 8^{\text {ac }}$ & $141 \pm 4^{\mathrm{A}}$ & $138 \pm 14^{\mathrm{A}}$ & $109 \pm 11^{\mathrm{B}}$ \\
FVL & $21 \pm 1^{\text {def }}$ & $18 \pm 1^{\mathrm{f}}$ & $20 \pm 2^{\text {ef }}$ & $50 \pm 6^{\mathrm{C}}$ & $37 \pm 2^{\mathrm{C}}$ & $36 \pm 7^{\mathrm{C}}$
\end{tabular}

Drought treatments were made by exposing plants to moderate $(60 \% \mathrm{FC})$ or severe ( $30 \% \mathrm{FC})$ drought stress for four weeks starting from 21 days after inoculation with Verticillium longisporum. Mock and/or VL-inoculated plants supplied with water at $100 \%$ FC were used as control. Mean \pm standard deviation data obtained from 30 plants of two independent experiments are presented. Mean values containing the same letter case superscript are not significantly different at $\mathrm{P}=0.05$. FC, field capacity. SM, SEM-05-500526 mock-inoculated. FM, Falcon mockinoculated. SVL, SEM-05-500526 VL-inoculated. FVL, Falcon VL-inoculated.

\subsubsection{Branching}

Unlike other agronomic traits, the unusual production of primary branches (lateral shoots arising from central main stem; Fig. 4.2) was solely affected by VL infection and its interaction with the genotype (Table 4.9). This indicates that the excessive production of lateral shoots in both genotypes was mainly induced by VL infection. This is clearly shown in a correlation analysis that showed a significant positive relationship of branching with disease severity, and a strong negative relationship with agronomic parameters and no correlation with all physiological parameters (Table 4.11). The induction of excessive branching due to VL infection regardless of genotype was observed two weeks after inoculation and remained evident until crop maturity (49 DPI). Compared to genotype SEM however, the effect was much stronger in cultivar Falcon which was also the case in other disease parameters (4.4.1). At 49 DPI and regardless of drought stress, VL-infection induced branching increased on the average by 2.3 and 4.1 fold in SEM and Falcon, respectively. Nevertheless, such a high induction of branching was not reflected in increased biomass yield particularly in the susceptible cultivar Falcon. This could be either due to the very small size of these branches or it might also be due to the fact that they undergo senescence shortly after their emergence. The effect of drought stress on branching either in mock- or VL-inoculated treatments was not significant during both early (Appendix 4.3) and late growth stages (Table 4.8). 


\subsubsection{Hypocotyl thickness}

Similar to yield parameters, stem thickness was the most prominent indicator of disease and drought effects, genotypic difference as well as of the interaction of these factors (Table 4.9). It was also the one that showed significant correlation with all other parameters considered in this study (Table 4.11). From the beginning, the two genotypes had genetically different mean stem thickness, SEM being thicker at the hypocotyl $(10.4 \mathrm{~mm})$ than Falcon $(9.4 \mathrm{~mm})$. Whether infected by VL or not, exposure of both genotypes to drought stress resulted in thinner plant stems, with no significant difference between moderate and severe drought stress treatments (Table 4.8). Compared to $100 \% \mathrm{FC}$, hypocotyl thickness at $60 \%$ and $30 \%$ FC in cultivar Falcon was significantly reduced by $10.8 \%$ and $19.1 \%$, respectively. In genotype SEM, the respective reduction percentages were 12.5 and 22.7. Similarly, the respective average stem thickness reduction during VL infection was 11.7 and $14.9 \%$ in SEM and 19.0 and $15.6 \%$ in Falcon. Even though not statistically significant, these data again tell that SEM is more sensitive to drought stress than cultivar Falcon. Furthermore, it was noticed that the rate of stem thickness reduction due to water stress was less in VL-inoculated treatments of both genotypes, indicating the reduced effect of drought due to $V L$ infection.

On the other hand, the effect of VL infection on stem thickness was only restricted to the susceptible cultivar Falcon causing 13.8, 21.7 and 10.0\% significant reduction at 100, 60 and 30\% FC, respectively. Interestingly, VL-resistance of genotype SEM was verified without any doubt as stems of infected plants remained as tough and vigorous as their control counterparts both under full irrigation as well as at moderate and severe water stress conditions (Table 4.8).

Table 4.8 Hypocotyl diameter and number of primary branches per plant measured from two Brassica napus genotypes exposed to drought stress and/or Verticillium longisporum infection at 49 DPI.

\begin{tabular}{ccccccc} 
& \multicolumn{3}{c}{ Number of primary branches/plant } & \multicolumn{3}{c}{ Hypocotyl diameter (mm) } \\
Treatments & $100 \% \mathrm{FC}$ & $60 \% \mathrm{FC}$ & $30 \% \mathrm{FC}$ & $100 \% \mathrm{FC}$ & $60 \% \mathrm{FC}$ & $30 \% \mathrm{FC}$ \\
\hline SM & $3.4 \pm 0.5^{\mathrm{b}}$ & $2.7 \pm 0.8^{\mathrm{b}}$ & $2.7 \pm 0.3^{\mathrm{b}}$ & $10.4 \pm 0.1^{\mathrm{a}}$ & $9.1 \pm 0.4^{\mathrm{bc}}$ & $8.1 \pm 0.2^{\mathrm{d}}$ \\
SVL & $6.5 \pm 0.4^{\mathrm{a}}$ & $6.8 \pm 1.3^{\mathrm{a}}$ & $6.6 \pm 0.5^{\mathrm{a}}$ & $9.6 \pm 0.3^{\mathrm{ab}}$ & $8.5 \pm 0.2^{\text {cd }}$ & $8.2 \pm 0.2^{\mathrm{d}}$ \\
FM & $2.0 \pm 0.2^{\mathrm{a}}$ & $2.0 \pm 0.3^{\mathrm{a}}$ & $1.6 \pm 0.3^{\mathrm{a}}$ & $9.4 \pm 0.2^{\mathrm{b}}$ & $8.4 \pm 0.1^{\mathrm{cd}}$ & $7.6 \pm 0.3^{\text {de }}$ \\
FVL & $7.7 \pm 0.3^{\mathrm{b}}$ & $7.8 \pm 0.8^{\mathrm{b}}$ & $7.0 \pm 1.2^{\mathrm{b}}$ & $8.1 \pm 0.3^{\mathrm{d}}$ & $6.6 \pm 0.7^{\mathrm{f}}$ & $6.9 \pm 0.2^{\text {ef }}$
\end{tabular}

Drought treatments were made by exposing plants to moderate $(60 \% \mathrm{FC})$ or severe $(30 \% \mathrm{FC})$ drought stress for four weeks starting from 21 days after inoculation with Verticillium longisporum. Mock and/or VL-inoculated plants supplied with water at $100 \%$ FC were used as control. Mean \pm standard deviation data obtained from 30 plants of two independent experiments are presented. Mean values containing the same superscript are not significantly different at $\mathrm{P}=0.05$. FC, field capacity. SM, SEM-05-500526 mock-inoculated. FM, Falcon mock-inoculated. SVL, SEM-05-500526 VL-inoculated. FVL, Falcon VL-inoculated. 


\subsubsection{Phenological growth stage}

Assessment of the phenological stages using the BBCH scales (Appendix 4.13) was begun three weeks after inoculation of seedlings with VL (21 DPI) and performed for five consecutive weeks (Fig. 4.13). At the time of inoculation, average growth stage of vernalized seedlings of both genotypes was BBCH 14 (four leaves unfolded). Three weeks after inoculation, control SEM plants had already attained inflorescence emergence stage $(\mathrm{BBCH} 50)$ while Falcon plants were at the stage of $\mathrm{BBCH} 45$ (stem elongation and development of lateral shoots). At 49 DPI, SEM plants in control treatments were ending the flowering stage (BBCH 67). At this time, Falcon was just beginning flowering (BBCH 62) (Fig. 4.8; Appendix 4.4). In general, the above results indicated that under normal growing conditions, genotype SEM grows relatively faster than Falcon.
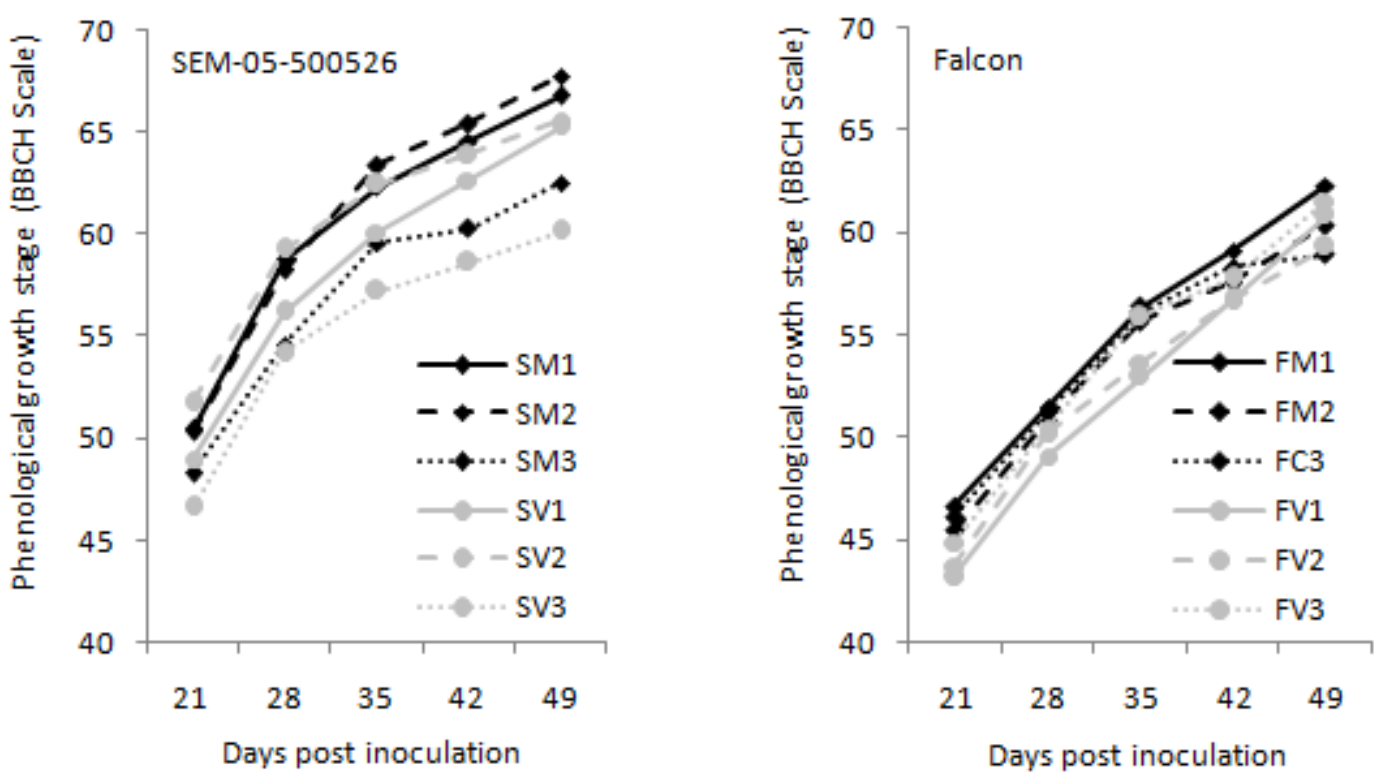

Figure 4.13 Phenological growth stages of two Brassica napus genotypes exposed to drought stress and/or Verticillium longisporum infection. Drought treatments were made by exposing plants to moderate (60\% FC) or severe $(30 \%$ FC) drought stress for four weeks starting from 21 days after inoculation with Verticillium longisporum. Mock and/or VL-inoculated plants supplied with water at $100 \%$ FC were used as control. Mean data obtained from 30 plants of two independent experiments are presented. FC, field capacity. S, genotype SEM-05500526. F, cultivar Falcon. M, mock-inoculated. V, infected with Verticillium longisporum. 1, 2 and $\mathbf{3}$ refer to watering at 100, 60 and 30\% field capacity respectively. Assessment was done using the $\mathrm{BBCH}$ scale.

The overall results showed that mock- and VL-inoculated had about the same growth stage, indicating no significant impact of VL infection on the phenological stages of both genotypes under greenhouse conditions. Regarding the impact of drought stress, significant differences between genotypes were observed. For cultivar Falcon, whether infected by VL or not, drought stress had no impact at all. Interestingly, as it is previously shown in physiological parameters, drought sensitivity of cultivar SEM was also clearly reflected in growth stage analysis where drought caused delayed flowering. At 49 DPI, 
mock-inoculated fully irrigated SEM plants attained $\mathrm{BBCH}$ 67, a stage where flowering is declining and the majority of petals are fallen. At this time point, severely drought stressed control SEM plants were still at the beginning stage of flowering (BBCH 63). During infection with $\mathrm{VL}$, a similar delay in flowering was caused by drought stress. Accordingly, VL-infected fully watered SEM plants at 49 DPI attained the full flowering stage (BBCH 65) while severely stressed plants were just at the initial stage of flowering (BBCH 60). No significant impact of moderate drought stress (watering at 60\% FC) was observed (Fig. 4.13; Appendix 4.4).

\subsubsection{Dry matter yield}

The potential effect of biotic or abiotic stress factors and the degree of plant resistance towards these stresses is ultimately measured by the plant capability in producing reasonable yield under such growing conditions. In the present study, the asessment of shoot and root dry biomass yield during early and late growth stages revealed significant contributions of genotype, disease, drought and their interactions in determining biomass yield accumulation (Table 4.9). Assessment of plant biomass at early (28 DPI; Appendix 4.5; Appendix 4.6; Appendix 4.7) and late (49 DPI) time points showed more or less similar trends. Hence, biomass yield quantified at the end of the experiement (49 DPI) is presented as follows.

\section{Effect of Verticillium longisporum on shoot and root DM yield}

Even though the magnitude of yield reduction was by far lower in the tolerant genotype, VL infection caused significant yied loss both in VL-suceptible and VL-tolerant plants (Fig. 4.14). Shoot dry matter (SDM) reduction due to VL infection at 100, 60 and 30\% FC in genotype SEM was 25.9, 35.1 and 9.9\%, respectively. Interestingly, SDM yield loss in SEM at 30\% FC was not significant indicating stable resistance of this genotype even under severe water deficit conditions. Neverthless, the loss due to infection at full irigation and moderate stress conditions was still higher than the one expected from a resistant genotype. From the evaluation of disease parameters, it is obvious that stunted growth (a symptom not induced under field conditions) was the major contributor to yield reduction in this genotype. In cultivar Falcon, susceptible to VL, a high and significant SDM yield reduction was caused by VL-infection resulting in 55.3, 63.2 and 51.4\% loss at 100, 60 and 30\% FC, respectively (Fig. 4.14; Table 4.13).

Similarly, assessment of root dry matter (RDM) yield showed that the effect of VL was extremely severe in Falcon but had no effect on SEM. Respective RDM accumulation in mock and VL-inculated SEM plants were 4.9 and $4.1 \mathrm{~g} /$ plant at 100\% FC, 4.0 and $3.7 \mathrm{~g} /$ plant at 60\% FC and 3.1 and $3.0 \mathrm{~g} /$ plant at 30\% FC,; indicating that VL infection has no effect during optimum water suply as well as under drought stress. 
Whereas in Falcon, substatial root biomass reduction was measured at all watering regimes resulting in $73.9,79.5$ and $62.8 \%$ reduction at 100,60 and $30 \%$ FC, respectively (Fig. 4.14; Table 4.13).

\section{Effect of drought stress on shoot and root DM yield}

Comparision of biomass formation under optimal growing conditions showed that genotype Falcon and SEM do not significantly differ in yield potential and provided similar quantity of yield. Upon addition of the drought factor however, SEM provided lower yield than Falcon indicating its relative sensetivity towards drought stress (Fig. 4.14). Accordingly, SDM in SEM was significantly reduced both at moderate (26.8\%) and severe (48.9\%) drought stress situations while it was only at severe drought stress condition (35.5\%) that significant shoot yield loss occured in cultivar Falcon. Concering root biomass yield, the effect of drought was noticed only at severe stress conditions. Here, moderate drought stress had no significant effect on root biomass production of both genotypes. Interestigly, enhanced drought sensitivity of genotype SEM was also observed on root biomass accumulation. Here, watering at $30 \%$ FC resulted in $36.8 \%$ reduction in SEM while it was only $25.2 \%$ in cultivar Falcon.

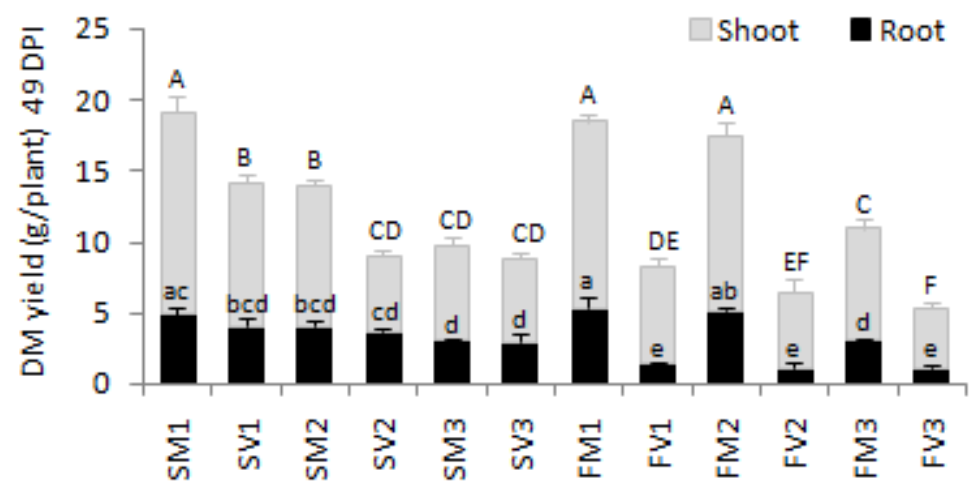

Figure 4.14 Shoot and root dry biomass yield of two Brassica napus genotypes subjected to Verticillium longisporum infection and/or drought stress conditions in a greenhouse experiment. Mock and/or VL-inoculated plants supplied with water at $100 \% \mathrm{FC}$ were used as control. Drought treatments were made by exposing plants to moderate $(60 \% \mathrm{FC})$ or severe $(30 \% \mathrm{FC})$ drought stress for four weeks starting from 21 days after inoculation with Verticillium longisporum. Mean data obtained from 30 plants of two independent experiments are presented. Bars indicate standard deviation. For each parameter, different letters on the bars with the same letter-case indicate significant differences between treatments at $\mathrm{P} \leq 0.05$. DM, dry matter. S, genotype SEM-05-500526. F, cultivar Falcon. M, mock-inoculated. V, infected with Verticillium longisporum. Treatment names followed by numbers 1, 2 and $\mathbf{3}$ refer to watering at 100, 60 and 30\% field capacity, respectively.

During infection with VL as well, shoot DM yield of both genotypes at moderate and severe drought stress conditions was significantly lower than the one obtained at full irrigation (Fig. 4.14). However, when comparing the rate of yield reduction in the presence or absence of VL-infection, the effect of severe drought stress (30\% FC) was relatively lower during VL infection compared to control conditions. 
In contrast, moderate drought stress (60\% FC) caused significantly higher losses with VL-infection compared to mock-inoculated treatments. Regarding accumulation of root biomass during VL infection, drought stress had no significant effect in both genotypes (Fig. 4.14).

\section{Total dry matter yield}

Total dry matter (TDM) reflecting the overall yield potential of the genotypes was obtained from the summation of shoot and root biomass yields of each treatment (Fig. 4.15). Mixed model ANOVA showed that all factors independently as well as thier interaction had significant effects on TDM accumulation (Table 4.9). Pearson's correlation analysis also showed a significant correlation of this trait with all disease, physiological and morphological parameters reflecting the strong link between plant stress response and yield accumulation (Table 4.11).

Irrispective of VL infection, TDM was significantly reduced by drought stress. The amount of loss at severe stress was signficantly higher compared to the loss occurring at moderate stress conditions. Genotypic differences with regard to drought stress tolerance were also reflected by TDM yield accumulation. In genotype SEM, TDM yield was significantly reduced both at moderate and severe drought stress conditions but in cultivar Falcon a significant reduction occured only at severe drought stress. Accordingly, the respective TDM yield reduction due to exposure to moderate and severe drought stress was 25.1 and $46.5 \%$ in SEM and 5.1 and $41.1 \%$ in Falcon, again suggesting better drought trolerance of genotype Falcon.

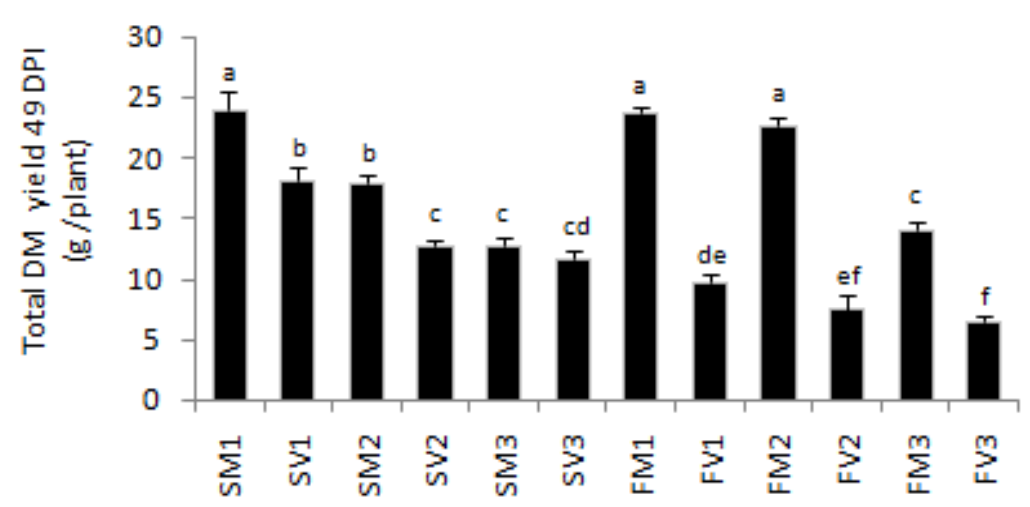

Figure 4.15 Total dry biomass yield of two Brassica napus genotypes subjected to Verticillium longisporum infection and/or drought stress in a greenhouse experiment. Mock- and/or VL-inoculated plants supplied with water at $100 \% \mathrm{FC}$ were used as control. Drought treatments were made by exposing plants to moderate (60\% FC) or severe $(30 \% \mathrm{FC})$ drought stress for four weeks starting from 21 days after inoculation with Verticillium longisporum. Mean data obtained from 30 plants of two independent experiments are presented. Bars indicate standard deviation. For each parameter, different letters on the bars indicate significant difference between treatments at $\mathbf{P} \leq 0.05$. S, genotype SEM-05-500526. F, cultivar Falcon. $\mathbf{M}$, mock inoculated. V, infected with Verticillium longisporum. Treatment names followed by numbers 1, $\mathbf{2}$ and $\mathbf{3}$ refer to watering at 100, 60 and $30 \%$ field capacity, respectively. 
During VL infection as well, TDM yield in genotype SEM was significantly reduced both at $60 \%$ and $30 \%$ FC. In cultivar Falcon however, even though relatively lower yields were obained from drought stress plants, the reduction was not significant (Fig. 4.15). This could be due to the stronger effect of the pathogen in this cultivar which might mask the effect of drought stress. Further comparison of TDM yield loss in mock- and VL-inoculated treatments showed that the impact of severe drought stress (30\% FC) was reduced during VL infection. For instance, TDM yield loss at 30\% FC in mock inoculated SEM and Falcon plants was 46.5 and $41.1 \%$, respectively. In VL-inoculated plants, these values were reduced to $29.6 \%$ in SEM and to $22.4 \%$ in Falcon. In contrast, a completely opposite situation was observed at moderate drought stress (60\% FC) condition. Here, the occurrence of drought during VL-infection caused more yield loss. As a result, the 25.1\% TDM yield loss occurred in mock-inoculated SEM was increased to $29.6 \%$ during infection with VL. Similarly, in cultivar Falcon, TDM loss was higher at the time when VL and moderate drought stress occurred simultanously (22.4\% loss) compared to only moderate drought stress (5\% loss) (Table 4.14).

Regarding the impact of VL infection, an obvious significant reduction of TDM yield occurred in both genotypes. However, compared to the suceptible cultivar Falcon, VL-infected SEM plants grown at all watering regimes provided consisently high yields. Accordingly, TDM yield in mock-inoculated SEM plants at 100, 60 and 30\% FC was 24.0, 18.0 and $12.8 \mathrm{~g} /$ plant, respectively. The respective yield during VL infection was 18.2, 12.8 and $11.7 \mathrm{~g} /$ plant. Interestingly, the yield obtained from mock- and VLinculated plants at $30 \%$ FC was almost the same indicating the consistency of disease resistance of SEM under drought conditions. In Falcon, the respective TDM yied in mock- and VL-incoculated plants was 23.8 and $9.7 \mathrm{~g} /$ plant at $100 \% \mathrm{FC}, 22.6$ and $7.5 \mathrm{~g} /$ plant at $60 \% \mathrm{FC}$ and 14.0 and $6.5 \mathrm{~g} /$ plant at $30 \% \mathrm{FC}$, with significant reduction at all watering regimes (Fig. 4.15).

In general, the effect of VL and drought stress on TDM accumulation of genotype SEM seems to be comparable. However when we look at the yield reduction due to VL infection, the contribution of stunting induced by VL-infection VL was more significant than any other disease symptom of VL. In Falcon, the suffering from VL infection was significantly higher than the effect of drought stress. Further comparison of the individual effects of either drought or V. longisporum with the combined effects, (i.e. comparison of mock-inoculated fully irrigated treatments with the rest of the treatments), the combined effect of VL and drought was by far stronger than any stress factor alone (Table 4.15). 
Table 4.9 F-values of analysis of variance for the effects of genotype, $V$. longisporum infection, drought stress and interaction factors on disease development, plant physiolgy and agronomic trtaits.

\begin{tabular}{|c|c|c|c|c|c|c|c|c|}
\hline \multicolumn{2}{|c|}{ Parameters } & G & $\mathrm{VL}$ & D & $\mathrm{G} * \mathrm{VL}$ & $G * D$ & $V L^{*} \mathrm{D}$ & $G * V L * D$ \\
\hline \multirow{5}{*}{ 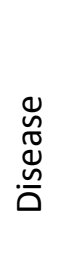 } & DF & 1 & 1 & 2 & 1 & 2 & 2 & 2 \\
\hline & AUDPC & $53.5^{*}$ & $398.5^{* * *}$ & $7.3^{\mathrm{ns}}$ & $62.3^{* *}$ & $1.4^{\mathrm{ns}}$ & $7.3^{* *}$ & $1.4^{\mathrm{ns}}$ \\
\hline & VL DNA & $286.0^{* * *}$ & $323.8^{* * *}$ & $2.0^{\mathrm{ns}}$ & $286.0^{* * *}$ & $1.8^{\mathrm{ns}}$ & $2.0^{\mathrm{ns}}$ & $1.8^{\mathrm{ns}}$ \\
\hline & RS & $568.6^{* * *}$ & $1227.5^{* * *}$ & $1.5^{\mathrm{ns}}$ & $568.6^{* * *}$ & $7.4^{* *}$ & $1.5^{\mathrm{ns}}$ & $7.4^{* *}$ \\
\hline & PB & $0.3^{\text {ns }}$ & $428.5^{* * *}$ & $1.4^{\mathrm{ns}}$ & $19.8^{* * *}$ & $0.4^{\mathrm{ns}}$ & $0.5^{\mathrm{ns}}$ & $0.5^{\mathrm{ns}}$ \\
\hline \multirow{5}{*}{ 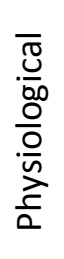 } & $\mathrm{E}$ & $0.1^{\mathrm{ns}}$ & $0.7^{\mathrm{ns}}$ & $52.1^{* * *}$ & $0.0^{\mathrm{ns}}$ & $2.5^{\mathrm{ns}}$ & $1.3^{\mathrm{ns}}$ & $0.1^{\mathrm{ns}}$ \\
\hline & gs & $0.0^{\mathrm{ns}}$ & $1.5^{\mathrm{ns}}$ & $30.3^{* * *}$ & $0.1^{\mathrm{ns}}$ & $1.3^{\mathrm{ns}}$ & $1.4^{\mathrm{ns}}$ & $0.0^{\mathrm{ns}}$ \\
\hline & A & $0.1^{\mathrm{ns}}$ & $0.2^{\mathrm{ns}}$ & $73.0^{* * *}$ & $0.1^{\mathrm{ns}}$ & $1.2^{\mathrm{ns}}$ & $2.6^{\mathrm{ns}}$ & $1.0^{\mathrm{ns}}$ \\
\hline & WUE & $2.4^{\mathrm{ns}}$ & $0.3^{\mathrm{ns}}$ & $24.7^{* * *}$ & $2.6^{\mathrm{ns}}$ & $1.3^{\mathrm{ns}}$ & $0.4^{\mathrm{ns}}$ & $3.6^{*}$ \\
\hline & RWC & $3.5^{\mathrm{ns}}$ & $0.8^{\mathrm{ns}}$ & $28.4^{* * *}$ & $3.1^{\mathrm{ns}}$ & $0.6^{\mathrm{ns}}$ & $0.0^{\mathrm{ns}}$ & $0.3^{\mathrm{ns}}$ \\
\hline \multirow{5}{*}{ 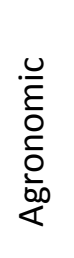 } & $\mathrm{PH}$ & $59.4^{* * *}$ & $273.5^{* * *}$ & $30.4^{* * *}$ & $134.0^{* * *}$ & $2.8^{\mathrm{ns}}$ & $5.2^{*}$ & $3.6^{\mathrm{ns}}$ \\
\hline & HD & $123.3^{* * *}$ & $72.8^{* * *}$ & $100.9^{* * *}$ & $17.5^{* * *}$ & $1.7^{\mathrm{ns}}$ & $7.6^{* *}$ & $1.0^{\mathrm{ns}}$ \\
\hline & RDM & $41.3^{* *}$ & $158.3^{* * *}$ & $42.3^{* * *}$ & $96.9^{* * *}$ & $0.5^{\text {ns }}$ & $11.6^{* *}$ & $5.7^{*}$ \\
\hline & SDM & $19.9^{* *}$ & $472.5^{*}$ & $86.2^{*}$ & $301.4^{* * *}$ & $26.4^{* * *}$ & $51.5^{* *}$ & $1.1^{\mathrm{ns}}$ \\
\hline & TDM & $30.9^{* *}$ & $409.1^{* * *}$ & $334.5^{* * *}$ & $105.5^{* * *}$ & $23.3^{* * *}$ & $62.5^{* * *}$ & $4.4^{*}$ \\
\hline
\end{tabular}

Drought treatments were applied by exposing plants to moderate $(60 \% \mathrm{FC})$ or severe $(30 \% \mathrm{FC})$ drought stress for four weeks starting from 21 days after inoculation with Verticillium longisporum (49 days post inoculation). ANOVA was performed using the PROC MIXED procedure (SAS 9.3, SAS Inst. 2002). P-values are indicated in superscripts and the values less than 0.05 are considered a significant effect. ${ }^{*}$, significant at $P=0.05$. ${ }^{* *}$, significant at $P=0.01$. ***, significant at $\mathrm{P}=0.001$. ns, not significant. DF, degree of freedom. $\mathbf{G}$, genotype. VL, Verticillium longisporum. $\mathbf{D}$, drought. AUDPC, net area under disease progress curve, VL DNA, Verticillium longisporum DNA in hypocotyl. RS, relative stunting. PB, number of primary branches per plant. E, transpiration rate. gs, stomatal conductance of $\mathrm{CO}_{2}$. A, photosynthesis rate. WUE, water use efficiency. LPC, leeaf proline content. HPC, hypocotyl proline content. RWC, leaf relative water content. PH, plant height. HD, hypocotyl diameter. SDM, shoot dry matter. RDM, root dry matter. TDM, total dry matter.

Table 4.10 F-value of analysis of variance for the effects of genotype, Verticillium longisporum infection, drought stress, and interaction factors on acumulation of free proline in leaf and hypocotyl tissue of Brassica napus.

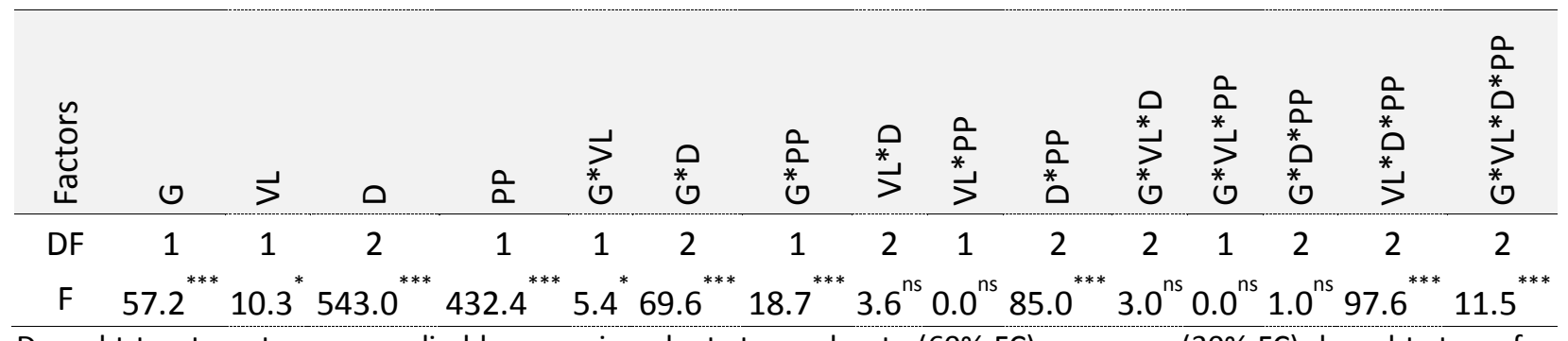

Drought treatments were applied by exposing plants to moderate $(60 \% \mathrm{FC})$ or severe $(30 \% \mathrm{FC})$ drought stress for four weeks starting from 21 days after inoculation with Verticillium longisporum (49 days post inoculation). ANOVA was performed using the PROC MIXED procedure (SAS 9.3, SAS Inst. 2002). P-values are indicated in superscripts and the values less than 0.05 are considered a significant effect. ${ }^{*}$, significant at $P=0.05$. ${ }^{* *}$, significant at $P=0.01$. $* * *$, significant at $\mathrm{P}=0.001$. ns, not significant. $\mathrm{DF}$, degree of freedom. $\mathrm{F}$, F-value. G, genotype. VL, Verticillium longisporum. D, drought. PP, plant part. 


\subsubsection{Correlation and regression analyses}

Pearson linear correlation analysis was conducted to analyse relationships within and between the different data categories. Among disease (AUDPC, VL DNA, stunting and branching) and agronomic parameters (plant height, hypocotyl diameter and DM yield), there were significant and strong positive correlations ( $r=0.74$ to 0.99 ). Regarding the relationship between disease and agronomic traits, again significant and strong, but negative correlations were found. Among these, the strongest relationship $(r=0.83$ to 0.91$)$ was observed between plant height and disease variables. In the same way, the relationship among physiological parameters (gas exchange, WUE, RWC and proline content) was strong and significant ( $r=0.52$ to 0.98). Depending on the type of parameters, the relationships were either positive or negative. The significance and strength of the relationships between physiological and agronomic variables was also variable depending on the type of the parameters. Of all the agronomic parameters, the one that showed the strongest significant correlation with physiological parameters was hypocotyl diameter. The positive correlation found between gas exchange and SDM was reasonably strong as well. On the other hand, the correlations between physiological parameters and plant height/RDM were either weak or insignificant. Interestingly, none of the disease parameters showed a significant correlation with any of the physiological measurements (Table 4.11). Similar results were obtained from data collected at $28 \mathrm{DPI}$ (Appendix 4.9).

Stepwise regression analysis is one of the most commonly used statistical tool which is applied to identify independent variables that significantly affect or most effectively predict the variation of a dependent variable (Gomez and Gomez, 1984). In the present study, this analysis was conducted to determine the relative contributions of genotype, disease and drought in affecting disease development, plant physiology and agronomic performance. Besides, a separate analysis was also performed to identify the most appropriate variables responsible for variations in TDM yield accumulation under drought and VL-infection conditions.

To determine the effects of the three experimental factors (genotype, disease and drought), stepwise regression was applied to 16 dependent variables representing disease, physiological and agronomic traits (Table 4.12). Before analysis, the three qualitative independent variables were coded as 0 and 1 (Gomez and Gomez, 1984). For convenience of analysis, data from fully watered and severely stressed treatments were used. Results showed that disease parameters were only significantly affected by VLinfection. Considering physiological parameters, except for the slight genotypic differences in proline synthesis, all physiological traits were only significantly affected by drought stress and no effect of VL on 
Table 4.11 Coefficients of Pearson's correlation ( $r$ ) describing relationships within and between disease, physiological and agronomic parameters measured from two Brassica napus genotypes exposed to drought stress and/or Verticillium longisporum infection 28 days after initiation of drought treatments (49 DPI).

\begin{tabular}{|c|c|c|c|c|c|c|c|c|c|c|c|c|c|c|c|c|}
\hline \multirow{2}{*}{\multicolumn{2}{|c|}{ Parameters }} & \multicolumn{4}{|c|}{ Disease } & \multicolumn{7}{|c|}{ Physiological } & \multicolumn{4}{|c|}{ Agronomic } \\
\hline & & AUDPC & VL DNA & RS & PB & $\mathrm{E}$ & gs & A & WUE & RWC & LPC & $\mathrm{HPC}$ & $\mathrm{PH}$ & $\mathrm{HD}$ & SDM & RDM \\
\hline \multirow{3}{*}{ 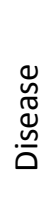 } & VL DNA & $0,89^{* * *}$ & & & & & & & & & & & & & & \\
\hline & RS & $0,95^{* * *}$ & $0,95^{* * *}$ & & & & & & & & & & & & & \\
\hline & PB & $0,88^{* * *}$ & $0,66^{* * *}$ & $0,76^{* * *}$ & & & & & & & & & & & & \\
\hline \multirow{7}{*}{$\begin{array}{l}\overline{\frac{\pi}{0}} \\
\frac{0}{00} \\
\frac{0}{0} \\
\frac{0}{0} \\
\frac{a}{0}\end{array}$} & $\mathrm{E}$ & $-0,05^{\mathrm{ns}}$ & $-0,11^{\mathrm{ns}}$ & $-0,02^{\mathrm{ns}}$ & $-0,05^{\mathrm{ns}}$ & & & & & & & & & & & \\
\hline & gs & $-0,10^{\text {ns }}$ & $-0,12^{\text {ns }}$ & $-0,05^{\mathrm{ns}}$ & $-0,10^{\text {ns }}$ & $0,98^{* * *}$ & & & & & & & & & & \\
\hline & A & $0,02^{\mathrm{ns}}$ & $-0,08^{n s}$ & $0,03^{\mathrm{ns}}$ & $0,05^{\mathrm{ns}}$ & $0,85^{* * *}$ & $0,79^{* * *}$ & & & & & & & & & \\
\hline & WUE & $-0,07^{\mathrm{ns}}$ & $0,04^{\mathrm{ns}}$ & $-0,05^{\text {ns }}$ & $-0,06^{\mathrm{ns}}$ & $-0,76^{* * *}$ & $-0,70^{* * *}$ & $-0,63^{* * *}$ & & & & & & & & \\
\hline & RWC & $0,14^{\mathrm{ns}}$ & $0,05^{\mathrm{ns}}$ & $0,12^{\mathrm{ns}}$ & $0,07^{\text {ns }}$ & $0,58^{* * *}$ & $0,52^{* *}$ & $0,69^{* * *}$ & $0,68^{* * *}$ & & & & & & & \\
\hline & LPC & $-0,15^{\mathrm{ns}}$ & $-0,04^{n s}$ & $-0,12^{\text {ns }}$ & $-0,13^{\text {ns }}$ & $-0,71^{* * *}$ & $-0,64^{* * *}$ & $-0,75^{* * *}$ & $0,75^{* * *}$ & $-0,79^{* * *}$ & & & & & & \\
\hline & $\mathrm{HPC}$ & $-0,22^{\text {ns }}$ & $-0,19^{\text {ns }}$ & $-0,24^{\mathrm{ns}}$ & $-0,13^{\text {ns }}$ & $-0,63^{* * *}$ & $-0,58^{* * *}$ & $-0,67^{* * *}$ & $0,70^{* * *}$ & $-0,80^{* * *}$ & $0,83^{* * *}$ & & & & & \\
\hline \multirow{5}{*}{ 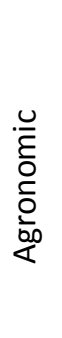 } & $\mathrm{PH}$ & $-0,89^{* * *}$ & $-0,88^{* * *}$ & $-0,91^{* * *}$ & $-0,73^{* * *}$ & $0,29^{*}$ & $0,30^{*}$ & $0,24^{\mathrm{ns}}$ & $-0,21^{\mathrm{ns}}$ & $0,13^{\text {ns }}$ & $-0,16^{\mathrm{ns}}$ & $0,07^{\mathrm{ns}}$ & & & & \\
\hline & $\mathrm{HD}$ & $-0,58^{* * *}$ & $-0,67^{* * *}$ & $-0,61^{* * *}$ & $-0,39^{*}$ & $0,59^{* * *}$ & $0,59^{* * *}$ & $0,65^{* * *}$ & $-0,37^{*}$ & $0,38^{*}$ & $-0,46^{* *}$ & $-0,34^{*}$ & $0,74^{* * *}$ & & & \\
\hline & SDM & $-0,73^{* * *}$ & $-0,66^{* * *}$ & $-0,68^{* * *}$ & $-0,67^{* * *}$ & $0,50^{* *}$ & $0,51^{* *}$ & $0,48^{* *}$ & $-0,35^{*}$ & $0,40^{*}$ & $-0,41^{*}$ & $-0,40^{*}$ & $0,88^{* * *}$ & $0,82^{* * *}$ & & \\
\hline & RDM & $-0,81^{* * *}$ & $-0,82^{* * *}$ & $-0,84^{* * *}$ & $-0,65^{* * *}$ & $0,35^{*}$ & $0,36^{*}$ & $0,33^{*}$ & $-0,20^{\text {ns }}$ & $0,26^{\mathrm{ns}}$ & $-0,26^{\text {ns }}$ & $-0,17^{\text {ns }}$ & $0,94^{* * *}$ & $0,77^{* * *}$ & $0,90^{* * *}$ & \\
\hline & TDM & $-0,76^{* * *}$ & $-0,72^{* * *}$ & $-0,73^{* * *}$ & $-0,68^{* * *}$ & $0,43^{* *}$ & $0,48^{* *}$ & $0,46^{* *}$ & $-0,32^{*}$ & $0,37^{*}$ & $-0,38^{*}$ & $-0,35^{*}$ & $0,91^{* * *}$ & $0,82^{* * *}$ & $0,99^{* * *}$ & $0,94^{* * *}$ \\
\hline
\end{tabular}

Correlation analysis was performed using PROC CORR procedure (SAS 9.3, SAS Inst. 2002). Minus signs indicate negative correlations. ns, not significant. *, significant at $\mathrm{P}=0.05$. ${ }^{* *}$, significant at $\mathrm{P}=0.01{ }^{* * *}$, significant at $\mathrm{P}=0.001$. DPI, Days post inoculation with Verticillium longisporum. AUDPC, net area under disease progress curve, VL DNA, Verticillium longisporum DNA in hypocotyl. RS, relative stunting. PB, number of primary branches per plant. E, transpiration rate. gs, stomatal conductance of $\mathrm{CO}_{2}$. A, photosynthesis rate. WUE, water use efficiency. LPC, leaf proline content. HPC, hypocotyl proline content. RWC, leaf relative water content. PH, plant height. HD, hypocotyl diameter. SDM, shoot dry matter. RDM, root dry matter. TDM, total dry matter. 
plant physiology was found. As expected, agronomic and yield traits were significantly influenced by all the three factors. Regarding plant height, most variation (49\%) was induced by VL infection, followed by genotype (16\%) and drought stress (11\%). Conversely, compared to VL (partial $r^{2}=0.10$ ), drought (partial $r^{2}=0.59$ ) and genotype (partial $r^{2}=0.22$ ) had stronger effects on hypocotyl diameter. Regarding yield parameters, the effect of $\mathrm{VL}$ and drought seems to be dependent on genotype and plant part (Table 4.12). Analysis of data collected at 28 DPI showed similar trends (Appendix 4.10).

Table 4. 12 Coefficients of determination (b), partial regression coefficients $\left(r^{2}\right)$, and $p$-values of stepwise regression analysis indicating the effects of genotype, $V$. longisporum infection and drought stress on disease, physiological and agronomic traits of two B. napus genotypes.

\begin{tabular}{|c|c|c|c|c|c|c|c|}
\hline \multirow{2}{*}{\multicolumn{2}{|c|}{ Parameters }} & \multicolumn{2}{|c|}{ Genotype } & \multicolumn{2}{|c|}{ V. longisporum } & \multicolumn{2}{|c|}{ Drought } \\
\hline & & $b$ & Partial $r^{2}$ & $b$ & Partial $r^{2}$ & $b$ & Partial $r^{2}$ \\
\hline \multirow{4}{*}{ 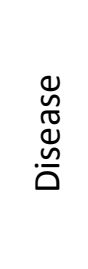 } & AUDPC & 20.6 & $0.11^{* * *}$ & 52.9 & $0.74^{* * *}$ & ns & ns \\
\hline & VL DNA & 468.1 & $0.30^{* * *}$ & 499.7 & $0.34^{* *}$ & ns & ns \\
\hline & Stunting & 25.1 & $0.21^{* * *}$ & 40.5 & $0.55^{* * *}$ & ns & ns \\
\hline & PB & ns & ns & 4.5 & $0.90^{* * *}$ & ns & ns \\
\hline \multirow{7}{*}{$\begin{array}{l}\overline{0} \\
\frac{0}{00} \\
\frac{0}{0} \\
\frac{0}{n} \\
\frac{c}{a}\end{array}$} & $E$ & ns & ns & ns & ns & -1.2 & $0.77^{* * *}$ \\
\hline & gs & ns & ns & ns & ns & 0.09 & $0.65^{* * *}$ \\
\hline & A & ns & ns & ns & ns & -6.0 & $0.87^{* * *}$ \\
\hline & WUE & ns & ns & ns & ns & 6.6 & $0.58^{* * *}$ \\
\hline & RWC & $\mathrm{ns}$ & ns & ns & ns & -11.9 & $0.69^{* * *}$ \\
\hline & LPC & -7.2 & $0.04^{* *}$ & ns & ns & 35.3 & $0.89^{* * *}$ \\
\hline & HPC & -10.1 & $0.14^{* *}$ & ns & ns & 21.4 & $0.61^{* * *}$ \\
\hline \multirow{5}{*}{ 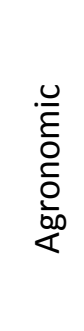 } & $\mathrm{PH}$ & -28.3 & $0.16^{* *}$ & -50.1 & $0.49^{* * *}$ & -24.2 & $0.11^{* *}$ \\
\hline & HD & -1.1 & $0.22^{* * *}$ & -0.7 & $0.10^{* * *}$ & -1.7 & $0.59^{* * *}$ \\
\hline & SDM & -2.2 & $0.05^{*}$ & -5.4 & $0.34^{* * *}$ & -6.3 & $0.45^{* * *}$ \\
\hline & RDM & -1.0 & $0.12^{* *}$ & -1.7 & $0.35^{* *}$ & -1.4 & $0.22^{* *}$ \\
\hline & TDM & -3.2 & $0.07^{*}$ & -7.2 & $0.35^{* * *}$ & -7.6 & $0.40^{* * *}$ \\
\hline
\end{tabular}

Mean data obtained from fully irrigated and severely stressed treatments of two independent experiments were used. Drought treatments (30\% FC) were applied for four weeks starting from 21 days after inoculation with Verticillium longisporum. AUDPC, net area under disease progress curve. VL DNA, Verticillium longisporum DNA in hypocotyl. RS, relative stunting. PB, number of primary branches per plant. E, transpiration rate. gs, stomatal conductance of $\mathrm{CO}_{2}$. A, photosynthesis rate. WUE, Water use efficiency. RWC, Leaf relative water content. LPC, leaf proline content. HPC, hypocotyl proline content. PH, plant height. HD, hypocotyl diameter. SDM, shoot dry matter. RDM, root dry matter. TDM, total dry matter; ns, not significant. *, significant at $\mathrm{P} \leq 0.05 .{ }^{* *}$, significant at $\mathrm{P} \leq 0.01 ; * *$, significant at $\mathrm{P} \leq 0.001$. 
To determine the functional relationships between yield contributing parameters and TDM yield, further stepwise regression analysis was performed. In this procedure, TDM was considered as the dependent variable against the rest of all disease, physiological and agronomic parameters. During each step of analysis, predicting variables with a partial regression coefficient $\geq 5 \%$ were retained in the model. Those predicting variables that show no or insignificant $(P \geq 0.05)$ relationships were used in following steps of analysis (Fig. 4. 16).

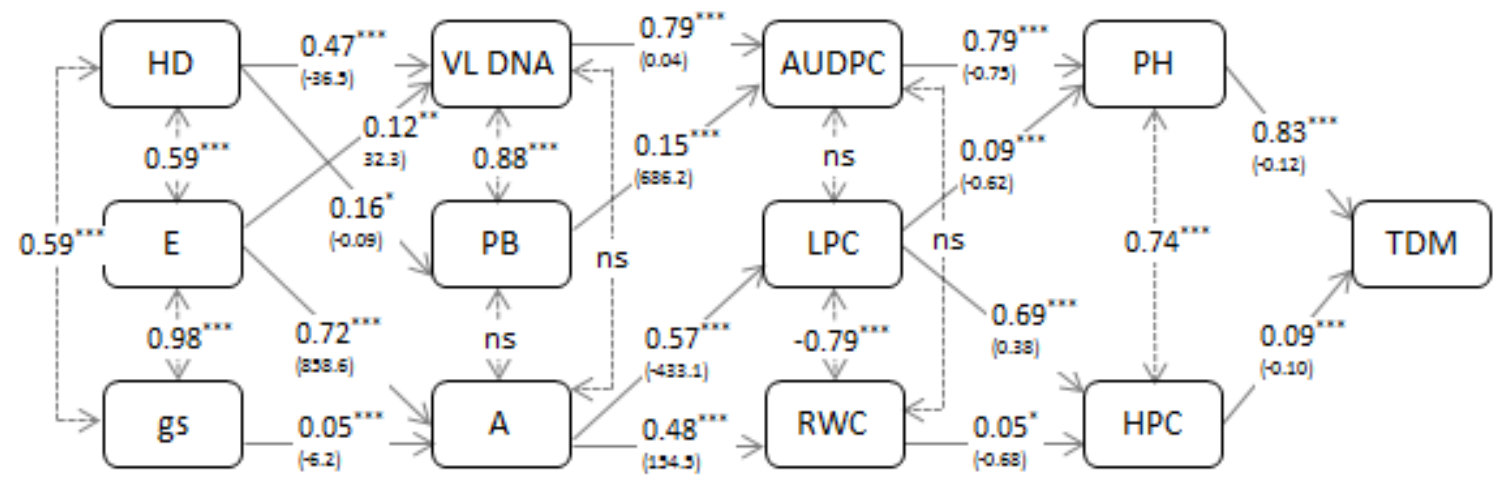

Figure 4.16 Sequential path model indicating stepwise regression coefficients of determination (b), partial regression coefficients $\left(r^{2}\right)$, Pearson's correlation coefficients $(r)$ and probability values of variables predicting total dry matter yield in Brassica napus genotypes subjected to drought stress and Verticillium longisporum infection.

Mean data obtained from 30 plants of two independent experiments were used for analysis. Drought treatments were applied for four weeks starting from 21 days after inoculation with Verticillium longisporum (49 DPI). Solid arrows show a stepwise regression analysis with partial regression coefficients. Coefficients of determination are indicated in brackets. Dotted arrows show coefficients of Pearson's correlation indicating relationships between the predicting variables. AUDPC, net area under disease progress curve. VL DNA, Verticillium longisporum DNA in hypocotyl. PB, number of primary branches per plant. E, transpiration rate. gs, stomatal conductance of $\mathrm{CO}_{2}$. A, photosynthesis rate. RWC, leaf relative water content. LPC, leaf proline content. HPC, hypocotyl proline content. PH, plant height. HD, hypocotyl diameter. TDM, total dry matter; ns, not significant; *, significant at $\mathrm{P}=0.05 ;{ }^{* *}$, significant at $\mathrm{P}=0.01 ; * * *$, significant at $\mathrm{P}=0.001$.

In the first order analysis, PH and HPC were kept in the model. Compared to the physiological parameter, HPC, which had little effect (9\%), and the disease parameter PH were responsible for most of the variation (83\%) in TDM indicating the stronger yield effect of VL infection compared to drought stress. Interestingly, the two parameters had a very strong significant positive correlation $(r=0.74)$. In the second order analysis, PH and HPC were considered as dependent variables and the stepwise regression was conducted with the rest of the predicting variables. Accordingly, AUDPC, LPC, and RWC that have shown significant relationships with $\mathrm{PH}$ and HPC were retained in the model. Here, the disease parameter AUDPC accounted for the largest variation (79\%) in PH but had no direct effect on HPC. The direct effect of LPC on PH was relatively small (9\%) but it contributed for the largest share (69\%) of variation in HPC (69\%). RWC had a minor effect (5\%) on HPC. Pearson correlation analysis showed no correlation between AUDPC and LPC or RWC whilst LPC had a strong negative correlation with RWC ( $r=$ 
-0.79). In the third order analysis which considered AUDPC, LPC and RWC as dependent variables, VL DNA, PB and A were retained in the model. VL DNA and PB justified 94\% of the variation in AUDPC. 57\% of the variation in LPC and 48\% in RWC were associated with variation in photosynthesis rate. Except for the strong correlation found between VL DNA and PB ( $r=88)$, no correlation was observed between the rest of disease and physiological parameters. In the fourth order analysis, a similar procedure was followed. The last three predicting variables remaining in the model were HD, E and gs. There were very strong positive correlations among these parameters. HD accounted for 47 and $16 \%$ of the variation in VL DNA and PB, respectively. E contributed for 72 and $12 \%$ of the variation in VL DNA and Photosynthesis. gs had very little direct effect (5\%) on photosynthesis rate (Fig. 4.16). The results from a similar analysis of data collected at 28 DPI can be found in Appendix 4.11.

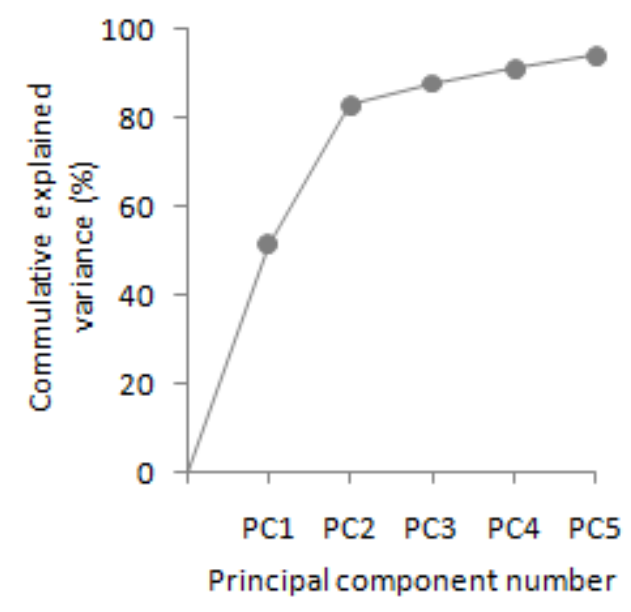

Figure 4.17 Scree plot of principal component analysis showing percentage of explained cumulative variance by principal components. Sixteen parameters measured from two $B$. napus genotypes subjected to drought stress and VL-infection were used in the analysis. Parameters included in the PCA: AUDPC, VL DNA, relative stunting, primary branches per plant, transpiration rate, stomatal conductance of $\mathrm{CO}_{2}$, photosynthesis rate, water use efficiency, leaf relative water content, leaf proline content, hypocotyl proline content, plant height, hypocotyl diameter, shoot dry matter, root dry matter, total dry matter.

\subsubsection{Principal component analysis}

Principal component analysis (PCA) is one of the multivariate statistical procedures used to compress data size, examine data structure and identify variables that best explain sample variability (Ringnér, 2008). In this study, PCA was employed to analyze the pattern of sixteen parameters and identify those that best explain variability in VL-resistant and VL-susceptible $B$. napus genotypes exposed to drought stress and VL-infection. Figure 4.18 shows the contribution of each principal component to total variance. A scores scatter plot (Fig. 4.18A) of the first two principal components that explain $83 \%$ of the total variation (Fig. 4.17) shows a clear separation of six groups of treatments arranged along principal component one (PC1) and two (PC2). The first three groups located along PC1 were separated due to disease and genotype factors (mock-inoculated, VL-inoculated resistant and VL-inoculated susceptible, Fig. 4.18A, circled). The separation of the second three groups situated along PC2 (shown in different colours) was mainly due to the drought factor (non-stressed, moderately-stressed and severelystressed), with further separation between control and infected treatments (Fig. 4.18A). The loading 
scatter plot that illustrates separation of the 16 parameters into four distinct groups (Fig. 4.18B). Physiological parameters were split into two distinct groups, the first comprising gas exchange and RWC and the second one proline and WUE. Agronomic (PH, HD, SDM, RDM and TDM) and disease (PB, VL DNA, RS and PB) parameters were grouped separately. Strong positive correlations within disease, physiological and agronomic variables, negative correlation between disease and agronomy parameters and another negative correlation between the two groups of physiological parameters are also shown (Fig. 4.18B). Furthermore, association of scores vs. loading plot indicates the contribution of all disease variables to distinct separation of infected treatments of the VL-susceptible cultivar. Proline content and WUE parameters seem to be the best variables to explain the variation due to severe drought stress in mock and VL-infected treatments of both genotypes.
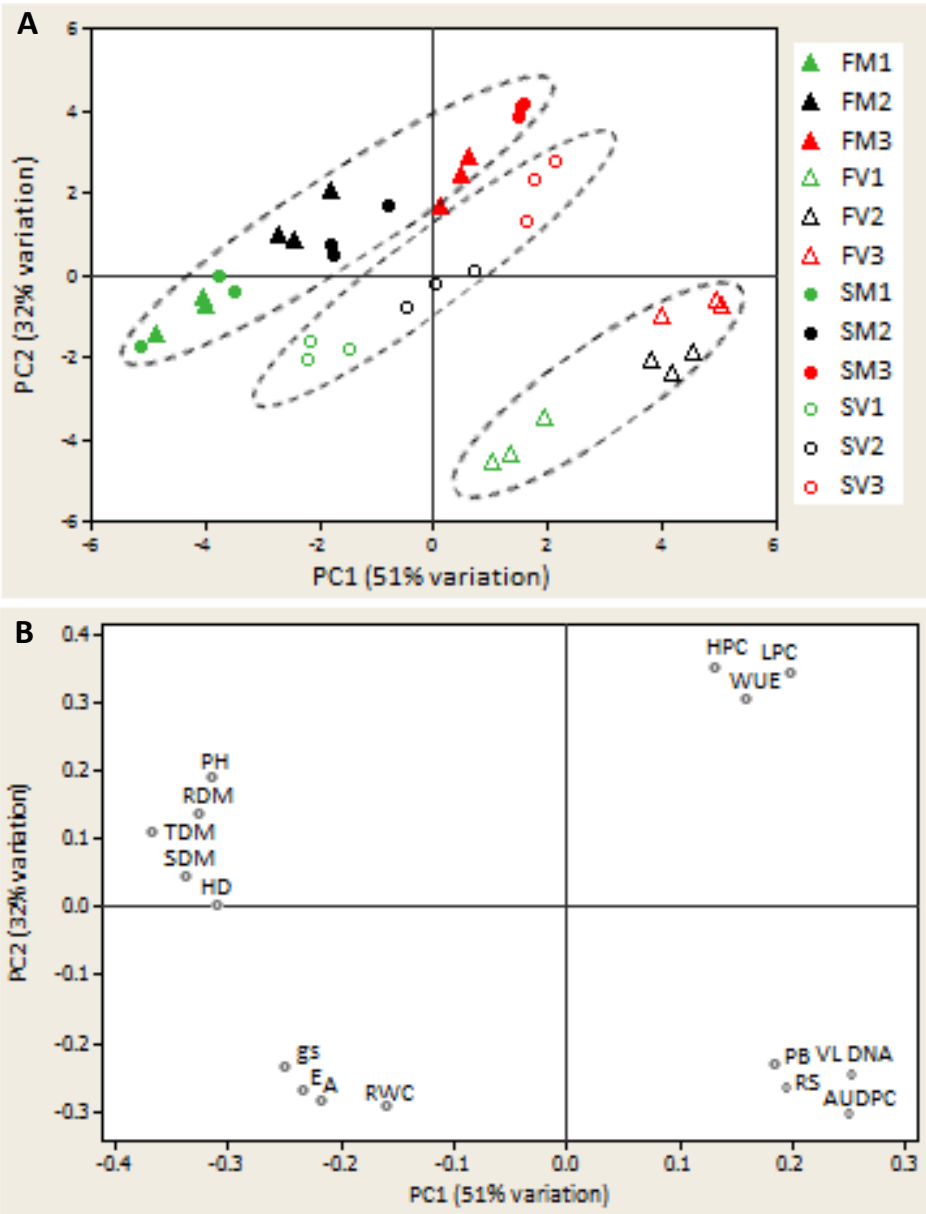

Figure 4.18 (A) Principal component analysis scores scatter plot of sixteen traits (disease, physiological and agronomic) measured in two $B$. napus genotypes grown under drought stress and Verticillium longisporum infection conditions. PC, principal component, S, genotype SEM-05-500526. F, cultivar Falcon. M, mock-inoculated. V, infected with Verticillium longisporum. Treatment names followed by the numbers 1, 2 and $\mathbf{3}$ refer to watering at 100, 60 and 30\% field capacity, respectively.

(B) Principal component analysis loading scatter plot of disease, physiological and agronomic parameters measured in two $B$. napus genotypes grown under drought stress and $V L$ infection conditions. Parameters included in the PCA: Area under disease progress curve (AUDPC),VL DNA in hypocotyl (VL DNA), relative stunting (RS), Primary branches per plant $(\mathrm{PB})$, transpiration rate $(\mathrm{E})$, stomatal conductance of $\mathrm{CO}_{2}$ (gs), photosynthesis rate $(A)$, water use efficiency (WUE), leaf relative water content (RWC)), leaf proline content (LPC), hypocotyl proline content (HPC), plant height (PH), hypocotyl diameter (HD), shoot dry matter (SDM), root dry matter (RDM), total dry matter (TDM). 


\subsection{Discussion}

\subsubsection{B. napus infection against $V$. longisporum under drought stress} AUDPC and $V$. longisporum DNA quantification by qPCR

Verticillium longisporum is a narrow host range pathogen mainly specialized on crucifers (Zeise and Tiedemann, 2002). A previous study on mechanisms of VL resistance in B. napus demonstrated that among other factors, accumulation of vascular occlusions is one of the key components contributing to VL resistance (Eynck et al., 2009b). Initially, it was assumed that despite serving as a mechanical barrier against fungal growth and dissemination, increased accumulation of vascular occlusions in hypocotyl xylem tissue of resistant $B$. napus genotypes might limit the translocation of water and mineral nutrients and thereby make plants more vulnerable to disease and drought stress. Disease evaluation results of the present comprehensive study however proved that the rate and level of Verticillium disease development in resistant B. napus plants is not affected by water supply. Hence, consistency of VLresistance under optimum watering and drought conditions not only proves that VL-resistance mechanisms have no additive negative consequence on plant performance during drought stress but also demonstrates effective operation of the quantitative VL- resistance mechanisms even under conditions of severe drought stress.

A weekly disease severity evaluation has shown that in addition to the late appearance of initial symptoms (21 DPI) in the resistant genotype SEM, the rate and level of symptom development over time and across all watering regimes was uniformly very low. For this genotype, average net AUDPC values at 28 and 49 DPI were 15 and 36, respectively. These are less than half of the values recorded from the susceptible cultivar Falcon. Quantification of VL DNA by quantitative real time PCR also revealed a significant suppression of VL growth in SEM, irrespective of water supply. Further comparison of fungal biomass between early (28 DPI) and late (49 DPI) time points showed reduced fungal biomass also at the later time point. With optimum water supply, the average VL DNA quantified at 28 DPI was $39.1 \mathrm{ng} / \mathrm{g}$ of dry hypocotyl tissues. When the plant reached the flowering stage (49 DPI), the quantity decreased to $27.1 \mathrm{ng} / \mathrm{g}$. In the presence of drought stress, fungal biomass during early and late growth stages remained similar. Even though in relatively small quantity, the detection of fungal DNA in hypocotyl tissue of the resistant genotype showed the occurrence of successful infection and indicates that resistance is not due to complete defense against infection. Eynck et al. (2009) have shown that VLresistance in $B$. napus is achieved by impaired shoot fungal spread caused by resistance factors induced after root penetration. In the present study, the reduction of fungal biomass at the later growth stage (49 DPI) might have also been due to the sufficient accumulation of resistance factors which halted 
fungal growth or even degrade some fungal structures while the plant continues growing and attaining increased size. In tomato plants infected with the wilt causing vascular pathogen Pseudomonas solanacearum, extensive and faster accumulation of the physical defence structure tyloses was observed in resistant varieties compared to susceptible plants (Grimault et al., 1994).

In contrast to SEM, successful infection in cultivar Falcon occurred shortly after infection and the typical symptoms of yellowing and stunted growth were distinctly observed relatively early, at 14 DPI. In addition to this, disease levels as measured by net AUDPC and VL DNA showed a trend of sharp increase over time. At $28 \mathrm{DPI}$, a net AUDPC value of 36.3, which is more than twice the values in SEM, was recorded. At $49 \mathrm{DPI}$, disease severity was increased by more than one fold (net AUDPC $=76.0$ ), which is again significantly higher than the values recorded from genotype SEM. In the presence of drought, a similarly high disease level as in fully watered treatments was recorded. Absolute quantification of fungal biomass by qPCR provided a better picture of the difference between susceptible and resistant genotypes. For instance, at optimal watering, 15-fold more VL DNA was quantified at 28 DPI in Falcon compared to SEM. Later at $49 \mathrm{DPI}$, this difference increased to 31-fold, indicating a sharp increase in fungal growth in cultivar Falcon vs. a decreasing tendency in SEM. Furthermore, qPCR results showed that levels of fungal DNA were positively correlated with the intensity of drought stress. A week after application of drought treatments (28 DPI), the respective average fungal DNA in dry hypocotyl tissue at 100, 60, and 30\% FC were 39.1, 30.4 and 35.2ng/g in SEM and 574.5, 659.9 and 826.6ng/g in Falcon. Three weeks later (49 DPI), the respective average fungal DNA at 100, 60, and 30\% FC was 27.1, 29.0 and $36.0 \mathrm{ng} / \mathrm{g}$ in SEM and 839.1, 1,032.4 and 1,096.4 ng/g in Falcon; indicating more pronounced effect of VL during drought stress particularly on the susceptible cultivar. The consistent inhibition of fungal growth across all watering levels in genotype SEM vs. the significant increased growth in cultivar Falcon during drought stress has led to a greater difference between the two genotypes. Significant impact of cultivar on the rate of VL colonization and symptom development has already been reported (Eynck et al., 2009a). Very thin and weak Falcon plants resulting from simultaneous stress from VL-infection and drought might favor VL to invade vascular tissue of susceptible cultivars and suggests a potential additive effect of VL-infection on susceptible B. napus varieties grown in drought-prone regions.

\section{Stunting effect}

One of the most peculiar greenhouse symptoms of VL infection in B. napus is stunted growth (Eynck et al., 2009a). Unlike the previously discussed disease parameters, significant stunting effect due to $V L$ infection was not restricted only to the susceptible genotype. The indiscriminate stunting effect of VL- 
infection suggests an infection induced change in plant metabolism that might cause hormonal imbalance leading to stunted growth and other abnormal phenotypes such as leaf shriveling and excessive production of side shoots (Fig. 4.2). Study on ethylene-mediated resistance in Arabidopsis showed the level of ethylene (a hormone known for inhibition of vegetative growth; promoting ripening and senescence) found to be increased in response to VL-infection (Johansson et al., 2006). Another study on the effect of VL-infection on plant nutrient relations in B. napus (Floerl et al., 2008) and Arabidopsis (Floerl et al., 2010) showed that severe stunting caused by VL-infection is not the consequence of nutrient limitation caused by xylem obstruction. Interestingly, significant stunting by VLinfection in the resistant genotype SEM was observed only in fully irrigated plants. Even the strong stunting effect (50\%) observed at $28 \mathrm{DPI}$ was further significantly reduced to $21 \%$ with further plant growth (49 DPI) indicating the recovery of plants over time. Failure of VL to induce stunting in the resistant genotype during drought stress provides another supportive evidence for stability of VLresistance under drought stress. On the other hand, this result might be also explained by the relatively high drought sensitivity of genotype SEM which showed strong stunting due to drought in mockinoculated plants compared to VL-infected treatments (Table 4.7). Concerning the VL-susceptible cultivar Falcon, the stunting effect of VL was extremely severe and not comparable with the drought effect. After all, VL-induced stunting was observed relatively early (14 DPI) and the degree or extent of stunting remained high over time. As a result, stunting effect of VL remained consistently significant until 49 DPI causing up to $74 \%$ reduction of plant height. Due to the severe stunting effect of VLinfection in this cultivar which probably masked the impact of drought stress, the clear determination of the stunting effect due to VL-infection under drought conditions was not possible.

Considering plant height as agronomic parameter, during the first week of exposure to drought stress (21-28 DPI), the effect on plant length was not significant in both genotypes. A significant reduction was first observed two weeks after the initiation of drought treatments (35 DPI) and remained evident until 49 DPI. Previous studies have shown that B. napus plants exposed to drought stress respond with shorter plant length (Qaderi et al., 2006; Shirani Rad and Zandi, 2012; Khalili et al., 2012). Interestingly, irrespective of genotype and growth stage, drought stress had no significant effect on plant height in VLinoculated plants. This could either be due to the relative strong stunting effect of VL, which masked the effect of drought stress, or due to a reduced impact of drought stress during VL-infection. It was also noticed that the impact of drought was relatively stronger on genotype SEM. Accordingly, moderate and severe drought stress significantly reduced plant height of SEM while it was only severe drought that 
caused significant reduction of plant height in Falcon. This is in strong agreement with the physiological measurements that revealed higher drought sensitivity of genotype SEM.

The consistent reaction of susceptible and resistant B. napus genotypes to VL-infection under different moisture conditions was reflected by correlation analysis that showed significant and strong positive relationships among phenotypic and molecular disease evaluation parameters (Table 4.11). The mixed model analysis of variance also showed that genotype, VL infection and their interaction were the major responsible factors that significantly affected all disease parameters (Table 4.9). According to stepwise multiple regression analysis, VL-infection and genotype were the most important factors that significantly contributed to the variation of all disease variables. For instance, $74 \%$ and $11 \%$ of the change in AUDPC was by VL and genotype, respectively. The respective contribution of these factors to the variation in VL DNA was 30 and $34 \% .55 \%$ and $21 \%$ of the variation in stunting was explained by VLinfection and genotype factors, respectively. $90 \%$ of the variation in branching was explained by VL infection. Drought alone and its interaction with VL or genotype and the combination of all the three factors have no significant impact on Verticillium disease development in B. napus, particularly in resistant genotypes (Table 4.12). Any of the disease evaluation variables showed significant correlation with physiological measurements (Table 4 .11). To date, there is no comprehensive study that looked at the impact of drought stress on the B. napus - V. longisporum interaction in general and the effect of drought stress on VL disease development in particular. Even the very few studies in Arabidopsis (Reusche et al., 2012; Reusche et al., 2014) were focusing only on the impact of infection on plant responses to drought stress. Nevertheless, related studies on other Verticillium species have shown that the impact of drought stress on disease development seems to be dependent on the crop and Verticillium species combination. According to Arbogast et al. (1999) the effect of moisture deficit stress on increased foliar senescence due to $V$. dahliae depends on potato cultivars. Pennypacker et al. (1991) showed reduced disease symptoms of Verticillium albo-atrum during drought stress in alfalfa. Bletsos et al. (1999) have reported an increased $V$. dahliae disease severity with decrease in irrigation frequency in eggplant.

\subsubsection{B. napus physiology during drought stress and infection with $V$. longisporum}

Physiological parameters were monitored in order to address the questions concerning $B$. napus-VL interaction under drought stress conditions. The first and foremost objective was to investigate whether VL-infection and the resulting accumulation of vascular occlusions have a negative impact on plant fitness under conditions of water deficit stress. In addition, the role of genotypic difference in 
determining physiological responses under disease and drought stress conditions was studied. To answer the above questions, several physiological parameters in plants grown under optimal conditions were compared to those exposed to either drought, VL-infection or both stress factors. Results obtained from the different physiological measurements as well as gene expression analysis consistently showed that $\mathrm{VL}$ infection in $B$. napus did not cause a significant change to most of the physiological variables considered. That is, except for proline synthesis and water use efficiency, all other physiological parameters measured in this study were only significantly affected by drought stress. Neither VL alone nor its interaction with drought or the genotype had a significant effect on expression of drought inducible genes (Fig. 4.12), gas exchange and RWC parameters (Table 4.9).

\section{Gas exchange and water use efficiency}

In general, regardless of genotype and VL infection, stepwise decrease in gas exchange (transpiration rate, stomatal conductance, photosynthesis rate) and increase in WUE was observed as water supply dropped from full watering $(100 \% \mathrm{FC})$ to $60 \%$ and $30 \%$ FC. Mixed model ANOVA showed that a significant reduction in gas exchange was only induced by drought. The remaining two factors and their interaction had no impact at all. Regarding WUE, in addition to the strong impact of drought, genotype $x$ $V L x$ drought interaction had a significant effect (Table 4.9). It was also noticed that the genotype SEM had a slightly higher WUE than Falcon. Besides, unlike VL-inoculated plants, 30\% FC watering in mockinoculated SEM plants significantly increased WUE (Fig. 4.7). Since increased WUE is a common evolutionary designed response of plants to drought stress (Blum, 2005), the above observations suggest two key points, namely the drought sensitivity of genotype SEM and the reduced impact of drought stress during infection with VL. In contrast to SEM, severe drought stress significantly increased WUE in VL-inoculated Falcon plants but not in mock-inoculated treatments, again suggesting a better reaction of this genotype to drought and the severe impact of VL-infection in this genotype as well. As it is shown for gas exchange parameters, VL infection has no significant impact on WUE of both genotypes (Table 4.9; Table 4.12). The impact of drought stress on B. napus gas exchange has been extensively investigated in various field and greenhouse studies. According to these studies, photosynthesis, transpiration and stomatal conductance are the major gas exchange parameters that are significantly reduced due to plant exposure to drought stress (Hashem et al., 1998; Naderikharaji et al., 2008; Sangtarash et al., 2009).

Regarding the impact of $\mathrm{VL}$, except for the slight reduction in transpiration rate and stomatal conductance in fully irrigated $(100 \% \mathrm{FC})$ treatments, VL-infection alone and its interaction with drought 
and genotype had no significant effect on gas exchange (Table 4.9; Table 4.12). Similar results have been reported by Floerl et al. (2008) who have shown no impact of VL-infection on photosynthesis and transpiration rates in B. napus. In another study with Arabidopsis, they also have shown that VLinfection has no negative impact on plant water and nutrient relation (Floerl et al., 2010). Similar observations have also been reported elsewhere in several crop species infected with other Verticillium species. Haverkort et al., 1990 found that $V$. dahliae alone and its interaction with drought had no impact on transpiration rate and stomatal conductance in early developmental stages of potato. In sunflower, stomatal conductance was unaffected by infection with V. dahliae (Sadras et al., 2000). Verticillium albo-atrum infection in alfalfa caused no significant reduction in stomatal conductance and photosynthesis (Pennypacker et al., 1991). Besides drought stress, different concomitant factors such as fungal toxins are suggested as causes of stomatal closure that lead to depression in photosynthetic activity in Verticillium albo-atrum infected tomato plants (Lorenzini et al., 1997). In another pathosystem, Thorne et al. (2006) have shown that grapevine infection by the xylem-limited bacterium Xylella fastidiosa caused no effect on stomatal conductance and transpiration rate. In the B. napus-VL interaction, it is evident that vascular colonisation of $V L$ and accumulation of occlusions is restricted to individual xylem vessels and adjacent vessels remained completely free and unaffected (Eynck et al, 2007). The presence of unaffected and fully functional vessels could provide adequate capacity for sufficient uptake of water and mineral nutrients. This could be one of the possible explanations why gas exchange is not affected by VL-infection and also why VL is not causing wilt symptoms in $B$. napus. Floerl et al. (2008) suggested that chlorotic and stunting symptoms of VL-infection in B. napus are not the result of limited water supply due to pathogen induced xylem obstruction. Induced accumulation of vessel occlusions due to infection with the bacterium Xylella fastidiosa was not causal for the water deficit and plant wilting symptoms in grapevine (Thorne et al., 2006). In contrast, a significant reduction of gas exchange due to drought stress induced by $V$. dahliae infection is reported in several crop species including pepper (Pascual et al., 2010), tomato (Bowden et al., 1990) and cotton (Hampton et al., 1990).

\section{Leaf relative water content}

As previously described, reduction in leaf relative water content (RWC) was observed due to water deficit and the effect was particularly significant at severe drought stress and at the later time point (Table 4.4). A week after initiation of drought treatments (28 DPI), RWC of stressed plants was slightly decreased but the reduction was not statistically significant except in infected Falcon plants. Since drought treatments began at $21 \mathrm{DPI}$ and plants at this time point were still small (BBCH 50; lower bud development stage), this might have led to low water demand and consequently to a low rate of 
transpiration. As a result, the soil moisture difference between normally watered and drought stressed treatments at 28 DPI might have not been large enough to show significant differences. Kumar and Elson (1992) have shown a significant effect of plant growth stage on leaf RWC of drought-stressed $B$. napus plants. At $49 \mathrm{DPI}$, however, due to large biomass production, applied water might be used quickly and reduction in leaf RWC was observed shortly after re-watering. Accordingly, $13 \%$ and $14 \%$ reduction in RWC was observed in mock- and VL-inoculated SEM plants, respectively. Interestingly, even at this time point, the reduction in cultivar Falcon was lower and insignificant, resulting in 10.3 and $10.1 \%$ loss at moderate and severe drought stress treatments, respectively. Depending on the plant cultivar or intensity of drought, up to $40 \%$ reduction of leaf RWC in B. napus have been reported in several studies (Ullah et al., 2012; Khalili et al., 2012; Sepehri and Golparvar, 2011; Good and Zaplachinski, 1994; Khalili et al., 2012). Regarding the disease factor, irrespective of plant age and genotype, no impact of VLinfection on RWC was observed (Table 4.9). Similarly, Reusche et al. (2014) recently reported no effect of the wilt inducing vascular pathogen $V$. dahliae on leaf water content of Arabidopsis. In pepper as well, leaf RWC remained unchanged until four weeks after inoculation with Verticillium dahliae (Goicoechea, et al, 2000). In contrast, Reusche et al. (2012) showed a rather positive impact of V. longisporum in Arabidopsis where infection provided improved leaf water content under drought stress condition.

\section{Proline content}

Accumulation of substantial amounts of free proline as a response to a wide range of biotic and abiotic stress is a common phenomenon in different groups of organisms (Delauney and Verma, 1993). In plants, stress-induced accumulation of proline has multiple positive roles in stress adaptation, recovery and signaling. It is involved in intracellular osmotic adjustment between cytoplasm and vacuole, protects photosynthetic organelles, stabilizes redox balance and influences programmed cell death which triggers HR during infection with avirulent pathogens, and regulates plant growth and development during stress conditions (Szabados and Savouré, 2010). In the present study, exposure of B. napus plants to drought stress caused up to 18-fold increase of proline accumulation. Drought-induced synthesis of free proline was positively correlated with the intensity of drought in plants in this study, with the highest amount quantified in plants supplied with water at 30\% FC. Similarly, a gradual pattern of increase in proline concentration with increase in intensity and duration of drought (Ghaffari et al., 2011; Omidi, 2010) and salinity stress (Saadia, et al., 2012) has previously been reported in B. napus.

Other factors responsible for variations in drought-induced proline synthesis were genotype and plant part. The initial leaf proline content was slightly higher in cultivar Falcon than in SEM. Drought stress 
however induced significantly higher proline synthesis in SEM, regardless of VL-infection and plant part (Fig. 4.10). It is possible that due to its sensitivity to drought, this genotype is responding with higher accumulation of proline. High proline accumulation does not necessarily reflect the level of drought tolerance in plants since it is rather a stress sensor and an indicator of the plant water status (Sundaresan and Sudhakaran, 1995; Hanson et al., 1977). This is in strong agreement with physiological, agronomic and disease evaluation results that clearly showed drought sensitivity and VL-resistance of this genotype. Expression analysis of the specific proline metabolism gene $\Delta 1$-pyrroline-5-carboxylate synthase1 (P5CS1) also revealed relatively increased expression of this gene in Falcon compared to SEM (Fig. 4.12). Saadia, et al. (2012) noted a maximum expression of the P5CS1 gene in a drought sensitive $B$. napus line. On the other hand, several studies showed strong association of high proline concentration and improved drought tolerance in several crop species including B. napus (Ghaffari et al., 2011; Saadia, et al., 2012), soybean (Silvente et al., 2012), alfalfa (Kang et al., 2011) and rice (Bunnag and Pongthai, 2013). Considering the impact of VL-infection in proline synthesis, it was noticed that drought induced proline accumulation in leaf and hypocotyl tissue was significantly higher in the VL-resistant genotype SEM (Fig. 4.10). In pepper, Goicoechea, et al. (2000) suggested an increased proline accumulation as a sensor of wilt damage caused by $V$. dahliae infection. In other pathosystems, high proline concentration is associated with resistance of Arabidopsis (Fabro et al., 2004) and tobacco (Senthil-Kumar and Mysore, 2012) against avirulent strains of Pseudomonas syringae via triggering of HR. In our B. napus-VL interaction however, since VL resistance does not involve induction of HR, the involvement of proline (as a scavenger of ROS) in plant resistance is not likely. The second interesting factor that showed significant differences in the amount of drought-induced proline accumulation was the plant part. Irrespective of any other factor considered in this experiment, it was found that the drought-induced total amount of proline was significantly higher in leaf than in hypocotyl tissue (Table 4.5; Fig. 4.10). As a key regulator of drought stress adaptation and signaling, it is not surprising that proline is present in high concentration in leaf tissues. Higher production of drought induced proline in the leaf than in stem and root tissue has been previously reported in potato (Ghorbanli et al., 2012) and in the ornamental plant Matthiola incana (El-Quesni et al., 2012).

\section{Comparison of drought induced changes in rate of physiological processes in the presence and absence of VL infection}

The most interesting and important observation regarding physiological parameters was the difference in physiological changes between mock- and VL-inoculated plants. Comparison of changes in gas exchange, hypocotyl proline and WUE due to drought stress under mock- and VL-inoculation conditions 
indicated that the impact of drought stress seems to be reduced during infection with VL. For instance, in mock-inculcated treatments, the rate of transpiration, stomatal conductance and photosynthesis at 30\% FC was reduced 4-, 6- and 2-folds in SEM and 4-, 5- and 3-fold in Falcon, respectively. During infection with VL, the respective reduction of the three gas exchange parameters was 2-, 3-, and 1-fold in SEM and 2-, 2-, and 1-fold in Falcon. In case of hypocotyl proline content, 10- and 18-fold increase due to severe drought stress was observed in mock-inoculated SEM and Falcon plants, respectively. Whenever the disease factor was added, proline accumulation was increased only by 7-fold in SEM and 4-fold in Falcon. Analysis of WUE also showed a similar trend for genotype SEM (Table 4.5). The reduced impact of drought stress during VL-infection in both VL-resistant and VL-susceptible $B$. napus genotypes may be associated with infection induced anatomical changes in xylem tissue. According to Eynck et al. (2007) and Eynck et al. (2009b), colonization of xylem vessels with VL and accumulation of VL-induced vascular occlusions in B. napus are restricted to individual vessels and other adjacent vessels remain completely uninfected and free of obstructions. The presence of a sufficient number fully functional vessel might provide efficient uptake and transport of water and this may explain why plants are not suffering from drought stress during VL-infection. Yadeta and Thomma et al. (2013) also suggested that if less numbers of vessels are closed by occlusions, the host plant will not suffer from drought stress. Furthermore, a recent study in Arabidopsis has shown enhanced drought tolerance of infected plants due to VL-induced de novo xylem formation (Reusche et al., 2014). Xu et al. (2008) showed viral infection induced an increase in osmoprotectant and antioxidant substances providing better drought tolerance to several crop species. Enhanced drought tolerance associated with mycorrhizal fungi has been reported in many crops such as wheat (Ellis et al., 1985; Abdel-Fattah and Abdul-Wasea, 2012), lettuce (Ruiz-Lozano et al., 1995), onion (Nelsen and Safir, 1982), common bean (Aroca et al., 2007), rosemary (Sánchez-Blanco et al., 2003), and pigeon pea (Qiao et al., 2011). Stimulation of increased accumulation of osmolites and sugars, improved nutrient uptake and root growth, reduced plant surface area of water loss etc. are among the mechanisms of mycorrhizal induced drought tolerance reported previously. It is also well known that an increase in xylem vessel density and diameter provides improved water absorption during drought which is closely associated with drought tolerance in tree (Qian and Ning, 2012) and annual crop species (Kulkarni et al., 2008).

\subsubsection{Expression of drought responsive genes during drought stress and infection with $\boldsymbol{V}$. longisporum}

The main intention of this study was to investigate the expression level of drought inducible genes in two $B$. napus genotypes that show contrasting resistance to VL-infection and to further analyze whether the pattern of gene expression is changing due to the formation of infection induced histological 
changes in xylem tissue. RT-PCR analysis of six different drought inducible genes showed up to 6-fold increase in gene expression caused by severe drought stress. This is in agreement with the results of most physiological measurements that showed a significant impact of drought on major physiological characters in mock- and VL- inoculated plants. The transcription factors DREB2 and CIPK1 that control the expression of stress-responsive genes via an ABA-independent pathway (Yang et al., 2010) and the P5SC1 gene which regulates proline biosynthesis in cytosol and chloroplast (Szabados and Savouré, 2010) showed the highest levels of expression. Previous studies in B. napus have shown that dehydration induced increased expression of DREB (Kagale et al., 2007) and P5SC1 (Saadia, et al., 2012; Xue et al., 2009) genes. Chen et al. (2012) reported increased expression of CIPK genes in B. napus due to salt stress and overexpression of these genes in Arabidopsis provided enhanced tolerance to salt stress. On the other hand, the expression of P5SC2, CBF17 and $\mathrm{HB} 6$ was relatively lower. P5CS2 is one of the key regulators of proline biosynthesis in mitochondria (Szabados and Savouré, 2010). CBF17 is a family of DREB1 transcription factors which is known to be mainly induced by cold stress (Gao et al., 2002; Savitch et al., 2005). Regarding HB6 which is a single copy gene in B. napus (Yu et al., 2005), not only that its expression was slightly increased by drought stress, but also its expression seems to be unaffected by drought stress when plants are infected with VL. According to Yu et al. (2005), this gene is constitutively induced by several abiotic factors in a time dependent manner. Genotypic comparisons also suggest that the accumulation of P5SC1, DREB2 and CIPK1 gene transcripts were relatively higher in Falcon than SEM. This is in agreement with physiological and yield assessment results that showed better performance of Falcon under drought conditions. Furthermore, the slightly reduced expression of HB6, P5SC1, DREB2, and P5SC2 genes observed at least in one of the genotypes might also suggest a reduced impact of drought stress during infection with VL.

\subsubsection{Single and combined effects of $V$. longisporum infection and drought stress on yield and agronomic traits}

\section{Branching}

Regarding production of primary branches, drought stress had very slight and insignificant effects (Table 4.8). Hashem et al. (1998) have showed similar results. Since plants were grown in a very confined root environment (limited to pot size), this condition might have physically limited root growth. Consequently, in order to balance the root-shoot ratio, plants might be forced to limit shoot vegetative growth such as branching. This may explain why numbers of branches in fully irrigated plants were not different from drought stressed treatments. In contrast, studies have shown that drought induced a significant reduction of primary branches in B. napus (Bilibio et al., 2011; Shirani Rad and Zandi, 2012). 
Sadaqat et al., (2003) found a high correlation between branching and seed yield of drought tolerant $B$. napus genotypes under drought stress conditions and suggested branching as best parameter for selection of high yielding canola varieties under drought. When we look at the effect of VL-infection, it induced excessive production of abnormal side branches both in susceptible and resistant genotypes. Unfortunately, due to the small size and short life span characteristics of these unusual branches, increased branching ( $>3$-fold) observed in infected plants was not reflected as improved biomass yield. A similar branching effect of VL infection in B. napus has been reported previously (Zeise, 1992). Häffner et al., (2010) have also shown two QTLs that control excessive branching effect of VL infection in Arabidopsis.

\section{Hypocotyl diameter}

Stem diameter, apart from genotypic differences, was remarkably influenced by drought, VL infection and all interaction factors (Table 4.8). Besides, stem diameter was the only trait (other than SDM) that showed a very strong correlation with the disease, physiological and other agronomic and yield measurements (Table 4.11). Genetically, the two genotypes had a significantly different stem thickness, SEM ticker than Falcon. VL-infection alone only reduced the hypocotyl diameter in the susceptible cultivar across all watering regimes. This may have been due to the utilization of xylem sap nutrients and the degradation of vessel walls by the fungus which might have led to reduced xylem diameter growth. Previous studies have shown that VL spends the largest part of its life cycle in the vascular environment (Eynck et al., 2007) using xylem sap constituents as nutrient source (see chapter 3 on xylem sap experiment; Floerl et al., 2010). Vascular phytopathogens including Verticillium species acquire their nutritional demand either directly from xylem sap, by degrading xylem vessel walls or inducing nutrient leakage from surrounding tissues (Yadeta and Thomma, 2013). In contrast, the hypocotyl of VL-infected SEM plants remained as thicker as in the control plants (Table 4.8), indicating consistency of VLresistance regardless of exposure to water stress. Under field conditions however, Gladders, (2009) showed no relationship between stem diameter and VL disease severity in UK. Considering the impact of drought stress, irrespective of genotype and VL-infection, the stem diameter of plants exposed to moderate and severe drought treatments was significantly reduced. It is obvious that drought exposed B. napus plants produce thinner stems (Bilibio et al., 2011; Qaderi et al., 2006). Principal component analysis also showed that stem diameter is mainly related to PC1 which contributed for much of the total variation (Appendix 4.12). In general, the above results suggest that among other things, the hypocotyl diameter can be considered as a potential trait for screening (greenhouse) B. napus varieties for $V$. longisporum resistance and possibly for drought tolerance as well. 


\section{Phenological growth stage}

The overall agronomic performance of the two genotypes under optimum and stressed growing conditions was further evaluated by weekly monitoring the phenological growth stages using the $\mathrm{BBCH}$ scale (Appendix 4.13). Interestingly, the results obtained from growth stage assessment are in line with the differential reactions of the two genotypes towards $V L$ and drought stress. The comparison of growth stages of the two $B$. napus genotypes revealed that irrespective of infection or drought stress, SEM grows slightly faster than Falcon. At the time of inoculation, vernalized seedlings of both genotypes had the similar growth stage (BBCH 14, four leaves unfolded). Three weeks later (21 DPI), SEM plants grown under optimum conditions began the inflorescence emergence stage (BBCH 50) while Falcon plants were completing the stem elongation stage $(\mathrm{BBCH} 47)$. One week later (28 DPI), SEM plants almost began flowering (BBCH 59) while Falcon plants just started the inflorescence emergence stage (BBCH 51). Assessment of growth stages until the $7^{\text {th }}$ week (49 DPI) regardless of infection and drought stress showed similar trends (Fig. 4.13; Appendix 4.4). The fast growth and development of genotype SEM, may contribute for early and rapid accumulation of resistance factors. This condition in turn might have made life very difficult for the pathogen particularly for the systemic dissemination in the shoot. Therefore, faster growth and accumulation of resistance factors, among other things, may be one of the factors contributing to VL-resistance in oilseed rape. In cotton, $V$. dahliae induced syntheses of antibiotic secondary metabolites (flavonoids) occur sooner and in greater concentration in resistant species than in susceptible materials (Bell and Stipanovic, 1978). More and rapid accumulation of the plant defence phytoalexin rishitin was shown in resistant tomato cultivars infected with Verticillium albo-atrum (Hutson and Smith, 1980) and Fusarium oxysporum (Elgersma and Liem, 1989). In another vascular pathosystem, Pritsch et al. (2000) have shown that PR genes were expressed earlier and stronger in a Fusarium graminearum resistant wheat cultivar than in a susceptible cultivar.

Considering the independent impact of drought stress, a clear genotypic difference which exactly reflects the differential physiological response of the two genotypes, was observed. Whether infected or not, drought stress had no visible impact on phenological growth stages of cultivar Falcon. This is in agreement with most physiological measurements where this cultivar has showed less sensitivity to drought stress. On the other hand, exposure of SEM plants to severe drought caused a reduced growth rate and finally resulted in delayed or inhibited flowering. Accordingly, the average growth stage at 49 DPI in mock inoculated fully-watered and severely-stressed SEM plants was BBCH 67 and BBCH 63, respectively. In the same way, the respective average growth stage of the VL-inoculated plants was $\mathrm{BBCH} 65$ and $\mathrm{BBCH} 60$ (Fig. 4.13; Appendix 4.4). Flowering stage is the most drought sensitive stage in 
oilseed rape and leads to high reduction in yield and oil content (Ahmadi and Bahrani, 2009; Hashem et al., 1998). Genotypic variations in drought resistance and drought sensitivity in the flowering stage of $B$. napus are reported in several studies (Naderikharaji et al., 2008; Champolivier and Merrien, 1996; Bouchereau et al., 1996; Good and Zaplachinski, 1994).

Regarding the pathogen factor, except for the slight delayed growth observed in VL-inoculated Falcon plants at $21 \mathrm{DPI}$ (Appendix 4.4), VL-infection in general had no observable impact on plant growth rate until the flowering stage (49 DPI). Nevertheless, early flowering is linked to a susceptible response to $V$. dahliae infection in Arabidopsis (Veronese et al., 2003; Steventon et al., 2001). It is also known that the switch from vegetative to flowering stage is a critical time for systemic spread of VL in B. napus (Zhou et al., 2006).

\section{V. longisporum infection and dry matter production}

The ultimate objective of this study was to examine the status of VL-resistance in B. napus during drought and to further find out whether there are costs associated with VL-resistance under drought condition and vice versa. As expected, DM yields were significantly reduced by VL-infection and drought stress. The severity of plant biomass loss was variable depending on plant genotype, type of stress and level of drought. Interestingly, compared to Falcon, VL-infection caused significantly lower shoot biomass yield in the VL-resistant genotype SEM with no impact on root biomass at all. Furthermore, VLinfection at severe drought stress did not affect shoot biomass yield of genotype SEM. Accordingly, infection-related SDM reduction under optimum watering was $25.9 \%$. At moderate drought stress, a slightly higher and again significant reduction (35.1\%) occurred. At severe stress level however, an extremely low (9.9\%) and insignificant reduction was recorded. Regarding RDM, the reduction at 100, 60 and $30 \%$ FC was $16.8,6.4$, and $4.2 \%$, respectively (Table 4.13), which were insignificant at all watering conditions (Fig. 4.14). The consistently low level of disease severity across all watering conditions (Fig. 4.6) and in contrast the strong plant height reduction due to VL infection (Table 4.6; Fig. 4.7) indicate that the significant SDM reduction in the VL-resistant genotype SEM is mainly attributable to the severe stunting effect of VL. As VL does not induce stunting under field conditions (Dunker et al., 2008), field grown resistant plants apparently perform much better and provide sufficient yield despite the prevalence of VL disease epidemics. In general, the reasonable SDM obtained from VL-infected fully and moderately irrigated plants, the lack of impact of infection on RDM and the SDM of severely stressed plants clearly show a stable and effective functioning of VL-resistance mechanisms regardless of the amount of water supply. 
Table 4.13 Dry matter yield reduction (\%) in two B. napus genotypes caused by Verticillium longisporum infection under optimum water supply and drought stress conditions.

\begin{tabular}{ccccccc} 
Yield & \multicolumn{3}{c}{ SEM } & \multicolumn{3}{c}{ Falcon } \\
parameters & $100 \% \mathrm{FC}$ & $60 \% \mathrm{FC}$ & $30 \% \mathrm{FC}$ & $100 \% \mathrm{FC}$ & $60 \% \mathrm{FC}$ & $30 \% \mathrm{FC}$ \\
\hline SDM & 25.9 & 35.1 & 9.9 & 55.3 & 63.2 & 51.4 \\
RDM & 16.8 & 6.4 & 4.2 & 73.9 & 79.5 & 62.8 \\
TDM & 24.1 & 28.7 & 8.6 & 59.5 & 66.9 & 53.9
\end{tabular}

Drought treatments were made by exposing plants to moderate $(60 \% \mathrm{FC})$ or severe ( $30 \% \mathrm{FC})$ drought stress for four weeks starting from 21 days after inoculation with Verticillium longisporum. Percent yield reduction due to VLinfection at 100, 60 and 30\% FC was calculated by comparing with the yield obtained from respective mockinoculated plants. Mean data obtained from 30 plants of two independent experiments are presented. FC, field capacity. SDM, shoot dry matter yield. RDM, root dry matter yield. TDM, total dry matter yield.

In contrast to SEM, DM reduction due to VL-infection in the VL-susceptible cultivar Falcon was significantly higher (Fig. 4.14). SDM reduction at 100, 60 and 30\% FC was 55.3, 63.2, and 51.2\%, respectively. Further high losses regardless of soil moisture conditions occurred in root biomass, resulting in $73.9,79.5$, and $62.8 \%$ significant reduction of RDM at 100,60 and $30 \%$ FC, respectively (Table 4.13). The significant yield impact of VL in cultivar Falcon was positively correlated with high disease levels across all watering levels. A yield loss assessment study has shown a significant yield impact of VL and a negative correlation between yield and VL disease severity in B. napus (Dunker et al., 2008). The relatively low rate of pathogen induced yield reduction occurred in severely-stressed tolerant and susceptible plants (Table 4.13) which also correlated with slightly lower AUDPC values (Fig. 4.6) suggesting that VL-drought interaction may favor the host plant. Possibly severe drought might impair VL growth whilst infection provides plants with better tolerance towards drought stress. As most fungi require free water or high moisture conditions for reproduction and dispersal, which is more crucial particularly for vascular pathogens like VL, drought usually has a negative direct impact on fungal growth (Desprez-Loustau, et al., 2006). On the other hand, it is also possible that the already induced significant yield reduction due to severe drought stress might have masked the effect of VL.

\section{Drought stress and dry matter production}

For the effect of drought stress on biomass accumulation, both moderate and severe drought conditions caused substantial yield loss, with more loss occurring at severe stress levels. Obviously, biomass or grain yield in oilseed rape is significantly affected under drought stress (Ashraf et al., 2013; Khalili et al., 2012; Bilibio et al., 2011; Siddiqui et al., 2008; Qaderi et al., 2006). Despite the significant biomass yield reduction in both genotypes, the effect of drought seems to be stronger in SEM than in Falcon. For genotype SEM, moderate and severe drought stress caused 26.8 and $48.9 \%$ significant loss of SDM, respectively. In cultivar Falcon however, SDM loss due to moderate drought stress was not significant 
(only 5\%). Nevertheless, SDM loss under severe stress conditions was significant (40.7\%) but still lower than in SEM. The above figures indicate that compared to Falcon, 22 and $8 \%$ more biomass yield loss occurred in SEM at 60 and 30\% FC treatments, respectively. Similar results have been observed during infection with VL as well. The respective percentage of SDM loss at moderate and severe drought in infected SEM plants was 35.9 and $37.9 \%$, while for Falcon it was 22.2 and $35.5 \%$ (Table 4.14). This is in agreement with the physiological measurements that showed less drought sensitivity of cultivar Falcon. Several studies have shown a differential genetic potential of $B$. napus cultivars to the accumulation of biomass yield under drought stress conditions (Ashraf et al., 2013; Hosseini et al., 2011; Dunker et al., 2008). Among the possible mechanisms associated with drought stress tolerance in $B$. napus, improved uptake and maintenance of increased concentration of essential nutrients (Ashraf et al., 2013; Siddiqui et al., 2008; Moradshai et al., 2004), stomatal resistance (Sadaqat et al., 2003), greater capacity in accumulation of proline along with other compatible solutes (Ghaffari et al., 2011; Saadia et al., 2012; Moradshai et al., 2004) and increased activity of ROS scavenging antioxidant enzymes (Abedi and Pakniyat, 2010) can be mentioned.

Further comparison of drought induced yield reduction in mock- vs. VL-infected SEM plants showed that severe drought caused $48.9 \%$ and $37.9 \%$ reduction in SDM in the absence and presence of the pathogen, respectively (Table 4.14). This means compared to mock inoculated plants, $11 \%$ less yield reduction occurred in plants infected with VL. For cultivar Falcon as well, 5\% less SDM yield reduction occurred when plants were infected with VL. These results may suggest a reduced impact of severe drought stress during VL infection which is in agreement with physiological data measurements. Furthermore, drought possibly limited growth and systemic spread of the fungus and this might have led to a slight contribution of infection for yield loss occurring during severe drought stress. In contrast, compared to optimum watering conditions, relatively high biomass yield reduction was recorded from VL-inoculated moderately stressed plants. In SEM, a moderate drought caused 9\% more SDM loss in VL-infected plants than in mock-inoculated treatments. Similarly in Falcon, 17\% more SDM loss occurred during infection with VL (Table 4.14). This is possibly because 60\% FC, that has caused significant effects on the host plant performance, might still be sufficient for growth and systemic spread of VL. Even though it is not known for VL, an earlier study on $V$. dahliae showed that infection on Brussels sprouts is more severe in wet soils than in dry conditions, with dry conditions delaying the onset of symptom development and reducing disease severity (Isaac, 1957). 
Compared to its large impact on shoot development, the influence of drought on root growth seems to be minimal. In mock inoculated treatments, 60\% FC drought stress had no impact at all. At 30\% FC however, a significant RDM reduction was recorded from both genotypes. Interestingly, drought in VLinoculated plants had no impact at all (Fig. 4.14). For cultivar Falcon, the severe impact of VL-infection that caused up to $80 \%$ root biomass loss (Table 4.13) might have masked the effect of drought stress and may perhaps explain why no significant difference was observed among VL-infected differentially watered treatments. In genotype SEM, root biomass remained unaffected by both drought and VLinfection. Since this genotype is resistant to VL, it is not surprising that root growth was not significantly affected by infection. However, it is interesting that the relative drought sensitivity of this genotype did not show in root growth. A recent study showed that irrespective of drought tolerance, root growth in $B$. napus cultivars increases with an increase in duration and intensity of drought stress (Ashraf et al., 2013). As observed in SDM yield analysis, a lower impact of severe drought stress on root biomass reduction in VL-infected treatments was noticed (Table 4.14).

Table 4.14 Dry matter yield reduction (\%) in two B. napus genotypes caused by moderate (60\% FC) and severe $(30 \% \mathrm{FC})$ drought stress in the presence and absence of Verticillium longisporum infection.

\begin{tabular}{|c|c|c|c|c|c|}
\hline \multirow{2}{*}{ Genotype } & \multirow{2}{*}{$\begin{array}{c}\text { Yield } \\
\text { parameters }\end{array}$} & \multicolumn{2}{|c|}{ Mock-inoculated } & \multicolumn{2}{|c|}{ VL-inoculated } \\
\hline & & $60 \%$ FC & $30 \%$ FC & $60 \%$ FC & $30 \%$ FC \\
\hline \multirow{3}{*}{$\sum_{\text {剻 }}$} & SDM & 26.8 & 48.9 & 35.9 & 37.9 \\
\hline & RDM & 18.3 & 36.8 & 8.1 & 27.3 \\
\hline & TDM & 25.1 & 46.5 & 29.6 & 35.5 \\
\hline \multirow{3}{*}{$\frac{\widetilde{0}}{\frac{0}{\pi}}$} & SDM & 5.1 & 40.7 & 22.0 & 35.5 \\
\hline & RDM & 4.8 & 25.2 & 42.4 & 18.0 \\
\hline & TDM & 5.1 & 41.1 & 22.4 & 33.0 \\
\hline
\end{tabular}

Drought treatments were made by exposing plants to moderate $(60 \% \mathrm{FC})$ or severe ( $30 \% \mathrm{FC})$ drought stress for four weeks starting from 21 days after inoculation with Verticillium longisporum. Percent yield reduction at $60 \%$ FC and $30 \%$ FC was calculated by comparing with the yields obtained from mock- or VL-inoculated $100 \% \mathrm{FC}$ treatments. Mean data obtained from 30 plants of two independent experiments are presented. FC, field capacity. SDM, shoot dry matter yield. RDM, root dry matter yield. TDM, total dry matter yield.

Overall, dry matter yield analysis indicated that severe drought may not favor VL-growth while at the same time VL-infection is possibly triggering changes in plant metabolism or architecture that provide plants with some level of tolerance against drought stress. However, this does not mean that simultaneous exposure of $B$. napus to drought stress and VL-infection has less impact than the independent effects of each stress factor. When either shoot, root or total DM yield of fully irrigated 
mock-inoculated plants (plants grown under normal or optimum conditions) were compared with the yields obtained from those subjected to either VL, drought or VL*drought, it is definitely true that the simultaneous occurrence of disease and drought stress causes significantly more yield reduction than what either of the stress factors are causing alone (Fig. 4.14 and 4.15). For instance, in genotype SEM, VL alone caused $24.1 \%$ of total biomass yield reduction. Moderate and severe drought independently caused 25.1 and $46.5 \%$ of total yield loss, respectively. Whenever both stress factors were applied simultaneously, the total yield loss due to the combined effect becomes $46.6 \%$ due to VL and moderate stress and 51.0\% due to VL and severe drought stress. Similarly, in cultivar Falcon total yield reduction due to VL infection alone was $59.5 \%$, due to moderate drought it was $5.1 \%$ and to severe drought $40.7 \%$. VL and moderate drought caused $68.6 \%$ loss. VL and severe stress caused $72.8 \%$ loss. Separate comparison of shoot and root biomass yields of both genotypes showed exactly the same trend (Table 4.15). From the above observations therefore, it may be concluded that despite the consistency of VLresistance under drought conditions and better drought resistance of VL-infected plants, the combined effect of VL and drought was by far stronger than any stress factor alone. This indicates the high significance of VL epidemics during drought seasons which can lead to a more pronounced yield loss.

Table 4.15 Single and combined effects of Verticillium longisporum infection and drought stress on dry matter yield reduction (\%) of two Brassica napus genotypes.

\begin{tabular}{ccccccccccc}
\hline $\begin{array}{c}\text { Yield } \\
\text { parameters }\end{array}$ & SM2 & SM3 & SV1 & SV2 & SV3 & FM2 & FM3 & FV1 & FV2 & FV3 \\
\hline SDM & 26.8 & 48.9 & 25.9 & 52.5 & 54.0 & 5.1 & 40.7 & 55.3 & 65.1 & 71.2 \\
RDM & 18.3 & 36.8 & 16.8 & 23.6 & 39.5 & 4.8 & 25.2 & 73.9 & 80.5 & 78.6 \\
TDW & 25.1 & 46.5 & 24.1 & 46.6 & 51.0 & 5.1 & 41.1 & 59.5 & 68.6 & 72.8 \\
\hline
\end{tabular}

Drought treatments were made by exposing plants to moderate $(60 \% \mathrm{FC})$ or severe $(30 \% \mathrm{FC})$ drought stress for four weeks starting from 21 days after inoculation with Verticillium longisporum. All yield reduction percent values were calculated by comparing with the yield obtained from mock-inoculated fully watered $(100 \% \mathrm{FC})$ treatments. Mean data obtained from 30 plants of two independent experiments are presented. FC, field capacity. SDM, shoot dry matter yield. RDM, root dry matter yield. TDM, total dry matter yield. SM, SEM-05-500526 mock-inoculated. FM, Falcon mock-inoculated. SV, SEM-05-500526 VL-inoculated. FV, Falcon VL-inoculated. Treatment names followed by the numbers 1, 2 and 3 refers to watering at 100, 60 and $30 \%$ field capacity, respectively.

\subsubsection{Correlation, regression and principal component analyses}

The outcome from correlation, regression and PCA analyses were considerably interconnected and provided strong evidence for consistent and differential responses of the two B. napus genotypes to VL infection and drought stress. With correlation analysis, the very strong relationships within disease, physiological and agronomic parameters, between disease and agronomic traits, and between physiological and agronomic traits were shown. Furthermore, the absence of association between VL- 
infection and changes in most physiological variables was also verified. PCA analysis in turn illustrated separate patterns of the three groups of variables and how strong they are linked to drought and disease treatments. With regression analysis, it was possible to confirm the significant but generally independent effect of pathogen and drought factors on disease and physiological traits, respectively. Apart from this, the significant effect of disease and drought on yield and agronomic traits as well as the significant contribution of the plant genotype in determining the variation in most variables were shown. With this analysis, it was also possible to identify key parameters (plant height and proline content) which are directly linked to most variations in dry mater biomass yield accumulation. Overall, the results of the three analyses suggest that $V L$ and drought may affect DM biomass yield of oilseed rape under greenhouse conditions by influencing plant systems that follow independent routes. 


\subsection{References}

Abdel-Fattah G. M. and Abdul-Wasea A. A. 2012. Arbuscular mycorrhizal fungal application to improve growthand tolerance of wheat (Triticum aestivum L.) plants grown in saline soil. Acta Physiologiae Plantarum, 34:267-277.

Abedi T. and Pankiyat H. 2010. Antioxidant enzyme changes in response to drought stress in ten cultivars of oilseed rape (Brassica napus L.). Czech Journal of Genetics and Plant Breeding, 46(1): 27-34.

Achuo E. A., Prinsen E. and Höfte M. 2006. Influence of drought, salt stress and abscisic acid onthe resistance of tomato to Botrytis cinerea and Oidium Neolycopersici. Plant Pathology, 55:178-186.

Ahmadi M. and Bahrani M. J. 2009. Yield and yield components of rapeseed as influenced by water stress at different growth stages and nitrogen levels. American-Eurasian Journal of Agricultural \& Environmental Sciences, 5(6): 755-61.

Anjum S. A., Xie X., Wang L., Saleem M. F., Man C. and Lei W. 2011. Morphological, physiological and biochemical responses of plants to drought stress. African Journal of Agricultural Research, 6(9): 2026-2032.

Arbogast M., Powelson M. L., Cappaert M. R., and Watrud L. S. 1999. Response of six potato cultivars to amount of applied water and Verticillium dahliae. Phytopathology, 89:782-788.

Aroca R., Porcel R. and Ruiz-Lozano J. M. 2007. How does arbuscular mycorrhizal symbiosis regulate root hydraulic properties and plasma membrane aquaporins in Phaseolus vulgaris under drought, cold or salinity stresses? New Phytologist, 173: 808-816.

Ashraf M. and Mehmood S. 1990. Response od four Brassica species to drought stress. Environmental and Experimental Botany, 30, (1):93-100.

Ashraf M., Shahbaz M. and Ali Q. 2013. Drought-induced modulation in growth and mineral nutrients in Canola (Brassica napus L.). Pakistan Journal of Botany, 45(1): 93-98.

Babadoost M., Chen W., Bratsch A. D. and Eastman C. E. 2004. Verticillium longisporum and Fusarium solani: two new species in the complex of internal discoloration of horseradish roots. Plant Pathology, 53:669-676.

Bates L. S. 1973. Rapid determination of free proline for water-stress studies. Plant and Soil, 39:205-207.

Bell A. A., and Stipanovic R. D. 1978. Biochemistry of disease and pest resistance in cotton. Mycopathologia, 65:91-106.

Berg G., Frankowski J. and Bahl H. 1999. Biocontrol of Verticillium wilt in oilseed rape by chitinolytic Serratia Plymuthica. Proceedings of $10^{\text {th }}$ International Rapeseed Congress. Canberra, Australia.

Bilibio C., J. Carvalho de A., Hensel O., Richter U. 2011. Effect of different levels of water deficit on rapeseed (Brassica napus L.) crop. Ciencia e Agrotecnologia, 35(4):672-684. 
Birnboim H.C. and Doly J. 1979. A rapid alkaline extraction procedure for screening recombinant plasmid DNA. Nucleic Acids Research, 7(6):1513-1523.

Bletsos F. A., Thanassoulopoulos C. C. and Roupakias D. G. 1999. Water stress and Verticillium wilt severity on eggplant (Solanum melongena L.). Journal of Phytopathology, 147(4):243-248.

Blum A. 2005. Drought resistance, water-use efficiency, and yield potential - are they compatible, dissonant, or mutually exclusive?. Australian Journal of Agricultural Research, 56:1159-1168.

Bouchereau A., Clossais-Besnard N., Bensaoud A., Leport L., and Renard M. 1996. Water stress effects on rapeseed quality. European Journal of Agronomy, 5:19-30.

Bowden R. L., Rouse D. I., and Sharkey T. D. 1990. Mechanism of photosynthesis decrease by Verticillium dahliae in potato. Plant Physiology, 94:1048-1055.

Brandfass, C., and Karlovsky, P. 2008. Upscaled CTAB-based DNA extraction and real-time PCR assays for Fusarium culmorum and F. graminearum DNA in plant material with reduced sampling error. International Journal of Molecular Sciences, 9: 2306-2321.

Bunnag S. and Pongthai P. 2013. Selection of rice (Oryza sativa L.) cultivars tolerant to drought stress at the vegetative stage under field conditions. American Journal of Plant Sciences, 4:1701-1708.

CABI/EPPO. 2011. Verticillium longisporum. Distribution maps of plant diseases, No. 1117. CAB International, Wallingford, UK.

Campbell C. L. and Madden L. V. 1990. Introduction to plant disease epidemiology. John Wiley and Sons, New York City, USA.

Carré P. and Pouzet A. 2014. Rapeseed market, worldwide and in Europe. Oilseeds and fats crops lipids, 21 (1) D102.

Champolivier L. and Merrien A. 1996. Effects of water stress applied at different growth stages to Brassica napus L. var. oleifera on yield, yield components and seed quality. European Journal of Agronomy, 5:153-160.

Chen L., Ren F, Zhou L, Wang Q. Q., Zhong H. and Li X. B. 2012. The Brassica napus Calcineurin B-Like 1/CBL-interacting protein kinase 6 (CBL1/CIPK6) component is involved in the plant response to abiotic stress and ABA signalling. Journal of Experimental Botany, 63(2):695-709.

Cong L., Chai T. Y and Zhang Y. X. 2008. Characterization of the novel gene BjDREB1B encoding a DREbinding transcription factor from Brassica juncea L. Biochemical and Biophysical Research Communications, 371:702-706.

Dalal M., Tayal D., Chinnusamy V. and Bansal K. C. 2009. Abiotic stress and ABA-inducible Group 4 LEA from Brassica napus plays a key role in salt and drought tolerance. Journal of Biotechnology, 139:137-145. 
Delauney A. J. and Verma D. P. S. 1993. Proline biosynthesis and osmoregulation in plants. The Plant Journal, 4(2):215-223.

Desprez-Loustau M. L., Marçais B., Nageleisen L. M., Piou D. and Vannini A. 2006. Interactive effects of drought and pathogens in forest trees . Annals of Forest Science, 63:597-612.

Din J., Khan S. U., Ali I. and Gurmani A. R. 2011. Physiological and agronomic responses of canola varieties to drought stress. The Journal of Animal \& Plant Sciences,21(1):78-82.

Dunker S., Keunecke H., Steinbach P. and von Tiedemann A. 2008. Impact of Verticillium longisporum on yield and morphology of winter oilseed rape (Brassica napus) in relation to systemic spread in the plant. Journal of Phytopathology, 156: 698-707.

Earl H. J. 2003. A precise gravimetric method for simulating drought stress in pot experiments. Crop Science, 43:1868-1873.

Elgesma D. M. and Liem J. I . 1989. Accumulation of phytoalexins in susceptible and resistant nearisogenic lines of tomato infected with Verticillium albo-atrum or Fusarium oxysporum f.sp. lycopersici. Physiological and Molecular Plant Pathology, 34: 545-555.

Ellis J. R., Larsen H. J. and Boosalis M. G. 1985. Drought resistance of wheat plants inoculated with vesicular-arbuscular mycorrhizae. Plant and Soil, 86(3):369-378.

El-Quesni F., Azza Mazhar A. M., Abd El Aziz N. G. and Metwally S. A. 2012. Effect of compost on growth and chemical composition of Matthiola incana (L.)R.Br. under different water intervals. Journal of Applied Sciences Research, 8(3): 1510-1516.

Ernst W.H. and Peterson P.J. 1994. The role of biomarkers in environmental assessment (4). Terrestrial plants. Ecotoxicology, 3:180-92.

Eynck C. 2008. Identification of resistance sources and characterization of resistance factors in Brassica species to Verticillium longisporum. PhD thesis. Georg-August-Universität Göttingen, Göttingen, Germany.

Eynck C., Koopmann B. and von Tiedemann A. 2009a. Identification of Brassica accessions with enhanced resistance to Verticillium longisporum under controlled and field conditions. Journal of Plant Diseases and Protection, 116(2), 63-72.

Eynck C., Koopmann B., Grunewaldt-Stoecker G., Karlovsky P. and von Tiedemann A. 2007. Differential interactions of Verticillium longisporum and V. dahliae with Brassica napus detected with molecular and histological techniques. European Journal of Plant Pathology, 118(3): 259-274.

Eynck C., Koopmann B., Karlovsky P. and von Tiedemann A. 2009b.Internal resistance in winter oilseed rape inhibits systemic spread of the vascular pathogen Verticillium longisporum. Phytopathology, 99:802-811. 
Fabro G., Kovács I., Pavet V., Szabados L. and Alvarez M. E. 2004. Proline accumulation and AtP5CS2 gene activation are induced by plant-pathogen incompatible interactions in Arabidopsis. Molecular Plant-Microbe Interactions, 17(4):343-50.

Farooq M., Wahid A., Kobayashi N., Fujita D. and Basra S. M. A. 2009. Plant drought stress: effects, mechanisms and management. Agronomy for Sustainable Development, 29(1): 185-212.

Fernandez-Garcia N., Hernandez M., Casado-Vela J., Bru R., Elortza F., Hedden P. and Olmos E. 2011. Changes to the proteome and targeted metabolites of xylem sap in Brassica oleracea in response to salt stress. Plant, Cell and Environment, 34:821-836.

Floerl S., Druebert C., Aroud H. I., Karlovsky P. and Polle A. 2010. Disease symptoms and mineral nutrition in Arabidopsis thaliana in response to Verticillium longisporum VI43 infection. Journal of Plant Pathology,92 (3), 693-700.

Floerl S., Druebert C., Majcherczyk A., Karlovsky P., Kües U. and Polle A. 2008. Defence reactions in the apoplastic proteome of oilseed rape (Brassica napus var. napus) attenuate Verticillium longisporum growth but not disease symptoms. BMC Plant Biology, 8:129.

Gao M. J., Allard G., Byass L., Flanagan A. M. and Singh J. 2002. Regulation and characterization of four CBF transcription factors from Brassica napus. Plant Molecular Biology, 49: 459-471.

Garrett K. A., Dendy S. P., Frank E. E., Rouse M. N., and Travers S. E. 2006. Climate change effects on plant disease: Genomes to ecosystems. Annual Review of Phytopathology, 44:489-509.

Ghaffari G., Toorchi M., Aharizad S. and Shakiba M. R. 2011. evaluation of traits related to water deficit stress in winter rapeseed cultivars. Universal Journal of Environmental Research and Technology, 1(3):338-350.

Ghorbanli M., Gafarabad M., Amirkian T. and Mamaghani B. A. 2012. Investigation of proline, total protein, chlorophyll, ascorbate and dehydroascorbate changes under drought stress in Akria and Mobil tomato cultivars. Iranian Journal of Plant Physiology, 3(2):651-658.

Goicoechea N., Aguirreolea J., Cenoz S. and Garcia-Mina J. M. 2000. Verticillium dahliae modifies the concentrations of proline, soluble sugars, starch, soluble protein and abscisic acid in pepper plants. European Journal of Plant Pathology, 106:19-25.

Gomez K. A. and Gomez A. A. 1984. Statistical procedures for agricultural research, $2^{\text {nd }}$ ed., John Wiley and Sons, New York.

Good A. G. and Zaplachinski S. T. 1994. The effects of drought stress on free amino acid accumulation and protein synthesis in Brassca napus. Physiologia Plantarum, 90: 9-14. 
Grimault V., Gélie B., Lemattre M., Prior P. and Schmit J. 1994. Comparative histology of resistant and susceptible tomato cultivars infected by Pseudomonas solanacearum. Physiological and Molecular Plant Pathology, 44:105-123.

Grzesiak M. T., Waligórski P., Janowiak F., Marcińska I., Hura K., Szczyrek P. and Głąb T. 2013. The relations between drought susceptibility index based on grain yield (DSI $\left.{ }_{G Y}\right)$ and key physiological seedling traits in maize and triticale genotypes. Acta Physiologiae Plantarum, 35(2):549-565.

Hampton R. E., Wullschleger S. D. and Oosterhuis D. M. 1990. Impact of Verticillium wilt on net photosynthesis, respiration and photorespiration in field-grown cotton (Gossypium hirsutum L.). Physiological and Molecular Plant Pathology, 37:271-280.

Hanson A. D., Nelsen C. E. and Everson E. H. 1977. Evaluation of free proline accumulation as an index of drought resistance using two contrasting barley cultivars. Crop Science.17 (5):720-726.

Hartung W., Peuke A. D. and Davies W. J. 1999. Abscisic acid - a hormonal long distance stress signal in plants under drought and salt stress. In: Pessarakali M. ed. Handbook of crop stress, $2^{\text {nd }}$ ed. New York, Marcel Dekker, 731-747.

Hashem A., Majumdar M. N. A., Hamid A. and Hossain M. M. 1998. Drought stress effects on seed yield, yield attributes, growth, cell membrane stability and gas exchange of synthesized Brassica napus L. Journal of Agronomy and Crop Science, 180(3): 129-136.

Haverkort A. J., Rouse D. I. and Turkensteen L. J. 1990. The influence of Verticillium dahliae and drought on potato crop growth. 1. Effects on gas exchange and stomatal behavior of individual leaves and crop canopies. Netherlands Journal of Plant Pathology, 96(5): 273-289.

Heale J. B. and Karapapa K. V. 1999. The Verticillium threat to Canada's major oilseed crop: Canola. Canadian Journal of Plant Pathology, 21(1):1-7.

Hosseini S. M. and Hassibi P. 2011. Effects of water deficit stress on several quantitative and qualitative characteristics of canola (Brassica napus L.) cultivars. Notulae Scientia Biologicae, 3(3):120-125.

Hutson R. A. and Smith I. M. 1980. Phytoalexins and tyloses in tomato cultivars infected with Fusarium oxysporum f.sp. lycopersici or Verlicillium albo-atrum. Physiological Plant Pathology, 17:245-257.

Ikeda K., Banno S., Watanabe K., Fujinaga M., Ogiso H., Sakai H., Tanaka H., Miki S., Shibata S. , Shiraishi T. and Fujimur M. 2012. Association of Verticillium dahliae and Verticillium longisporum with Chinese cabbage yellows and their distribution in the main production areas of Japan. Journal of General Plant Pathology, 78(5): 331-333.

Inderbitzin P., Davis R. M., Bostock R. M. and Subbarao K. V. 2011a. The Ascomycete Verticillium longisporum is a hybrid and a plant pathogen with an expanded host range. PLOS ONE, 6(3): e18260. 
Inoue H., Nojima H. and Okayama H. 1990. High efficiency transformation of Escherichia coli with plasmids. Gene, 96(1):23-28.

Isaac I. 1957. Verticillium wilt of Brussels sprout. Annals of Applied Biology, 45(2): 276-283.

Jensen C. R., Mogensen V. O., Mortensen G., Fieldsend J. K., Milford G. F. J., Andersen M. N., and Thage J. H. 1996. Seed glucosinolate, oil and protein contents of field-grown rape (Brassica napus L.) affected by soil drying and evaporative demand. Field Crops Research, 47:93-105.

Johansson A., Goud J. C. and Dixelius C. 2006. Plant host range of Verticillium longisporum and microsclerotia density in Swedish soils. European Journal of Plant Pathology, 114:139-149.

Jun-Wei W., Feng-Ping Y., Xu-Qing C., Rong-Qi L., Li-Quan Z., Dong-Mei G., Xiao-Dong Z., Ya-Zhen S. and Gai-Sheng Z. 2006. Induced expression of DREB transcriptional factor and study on its physiological effects of drought tolerance in transgenic wheat. Acta Genetica Sinica, 33(5):468476.

Kagale S., Divi U. K., Krochko J. E., Keller W. A. and P. Krishna. 2007. Brassinosteroid confers tolerance in Arabidopsis thaliana and Brassica napus to a range of abiotic stresses. Planta, 225:353-364.

Kang Y., Han Y., Torres-Jerez I., Wang M., Tang Y., Monteros M. and Udvardi M. 2011. System responses to long-term drought and re-watering of two contrasting alfalfa varieties. The Plant Journal, 68:871-889.

Karapapa V. K., Bainbridge B. W. and Heale J. B. 1997. Morphological and molecular characterization of Verticillium longisporum comb. nov., pathogenic to oilseed rape. Mycological Research, 101:1281-1294.

Khalili M., Aboughadareh A. P., Naghavi M. R. and Talebzadeh S. J. 2012. Response of spring canola (Brassica napus L.) genotypes to water deficit stress. International Journal of Agriculture and Crop Sciences, 4 (21):1579-1586.

Knüfer J. 2013. Improvement of winter oilseed rape resistance to Verticillium longisporum - Assessment of field resistance and characterization of ultrastructural plant responses. PhD thesis. GeorgAugust-Universität Göttingen, Göttingen, Germany.Kulkarni M., Borse T. and Chaphalkar S. 2008. Mining anatomical traits: A novel modelling approach for increased water use efficiency under drought conditions in plants. Czech Journal of Genetics and Plant Breeding , 44(1):11-21.

Kumar A. and Singh D. P. 1998. Use of physiological indices as a screening technique for drought tolerance in oilseed Brassica species. Annals of Botany, 81:413-420.

Lee S. C., Lim M. H., Kim J. A., Lee S. I., Kim J. S., Jin M., Kwon S. J., Mun J. H., Kim Y. K., Kim H. U., Hur Y. and Park B. S.2008. Transcriptome analysis in Brassica rapa under the abiotic stresses using Brassica 24K oligo microarray. Molecules and Cells, 26(6):595-605. 
Lehner B. and D̈oll P. 2001. Europe's droughts today and in the future. In Eurowasser - Model-based Assessment of European Water Resources and Hydrology in the Face of Global Change, Kassel World Water Series Report No 5, Lehner B., Henrichs T., D̈oll P and Alcamo J. (eds). Kassel, Germany. Leino M. 2006. Fungal diseases on oilseed rape and turnip rape. Norrköping, Sweden.

Lindemose S., O'Shea C., Jensen M. K. and Skriver K. 2013. Structure, function and networks of transcription factors involved in abiotic stress responses. International Journal of Molecular Sciences, 14:5842-5878.

Lorenzini G., Guidi L., Nali C., Ciompi S. and Soldatini G. F. 1997. Photosynthetic response of tomato plants to vascular wilt Diseases. Plant Science, 124:143-I52.

Mahmood S., A. Hussain, Z. Tabassum and F. Kanwal. 2004. Comparative performance of Brassica napus and Eruca sativa under water deficit conditions: An assessment of selection criteria. Journal of Research (Science), 14(4): 439-446.

Mithen R.. 1992 . Leaf glucosinolate profiles and their relationship to pest and disease resistance in oilseed rape. Euphytica, 63:71-83.

Moghadam H. R. T., Zahedi H., Ghooshchi F. 2011. Oil quality of canola cultivars in response to water stress and super absorbent polymer application. Pesquisa Agropecuária Tropical (Agricultural Research in the Tropics), 41: 579-586.

Moradshahi A., Eskandari S. B. and Kholdebarin B. 2004. Some physiological responses of canola (Brassica napus L.) to water deficit stress under laboratory conditions. Iranian Journal of Science \& Technology, Transaction A, 28(A1):43-50.

Müller T., Lüttschwager D. and Lentzsch P. 2010. Recovery from drought stress at the shooting stage in oilseed rape (Brassica napus). Journal of Agronomy and Crop Science, 196(2): 81-89.

Naderikharaji R., Pakniyat H. and Biabani A. R. 2008. Effect of drought stress on photosynthetic rate of four rapeseed (Brassica napus) cultivars. Journal of Applied Sciences, 8(23): 4460-4463.

Nelsen C. E. and Safir G. R. 1982. Increased drought tolerance of mycorrhizal onion plants caused by improved phosphorus nutrition. Planta, 154:407-413.

Neupane S., Andersson B., Högberg N., Ihrmark K. and Alström S. 2013. Fungal communities associated with field grown oilseed rape (Brassica napus L.) - their possible role in early crop establishment. Acta Agriculturae Scandinavica, Section B-Soil \& Plant Science, 63(3):241-252.

Newton A. C. and Young I. M. 1996. Temporary partial breakdown of Mlo-resistance in spring barley by the sudden relief of soil water stress. Plant pathology, 45:973-977. 
Obermeier C., Hossain M. A., Snowdon R., Knüfer J., von Tiedemann A. and Friedt W. 2013. Genetic analysis of phenylpropanoid metabolites associated with resistance against Verticillium longisporum in Brassica napus. Molecular Breeding, 31:347-361.

Omidi H., 2010. Changes of proline content and activity of antioxidative enzymes in two canola genotype under drought stress. American Journal of Plant Physiology, 5: 338-349.

Park B. J., Liu Z., Kanno A., Kameya T. 2005. Genetic improvement of Chinese cabbage for salt and drought tolerance by constitutive expression of a B. napus LEA gene. Plant Science, 169:553-558.

Pascual I., Azcona I., Morales F., Aguirreolea J. and Sanchez-Dıaz M. 2010. Photosynthetic response of pepper plants to wilt induced by Verticillium dahliae and soil water deficit. Journal of Plant Physiology, 167:701-708.

Pennypacker B. W., Leath K. T. and Hill R. R. Jr. 1991. Impact of drought stress on the expression of resistance to Verticillium albo-atrum in alfalfa. Phytopathology, 81:1014-1024.

Pertot I. and Elad Y.. 2012. Climate change impact on plant pathogens and plant diseases, Envirochange project booklet. Fondazione Edmund Mach. San Michele, Italy.

Pfaffl M. W. 2001. A new mathematical model for relative quantification in real-time RT-PCR. Nucleic Acids Research, 29(9):e45.

Pritsch C., Muehlbauer G. J., Bushnell W. R., Somers D. A. and Vance C. P. 2000. Fungal development and induction of defense response genes during early infection of wheat spikes by Fusarium graminearum. Molecular Plant-Microbe Interactions, 13(2):159-169.

Qaderi M. M., Kurepin L. V. and Reid D. M. 2006. Growth and physiological responses of canola (Brassica napus) to three components of global climate change: temperature, carbon dioxide and drought. Physiologia Plantarum, 128:710-721.

Qian X. and Ya-Ning C. 2012. Response of anatomy and hydraulic characteristics of xylem stem of Populus euphratica Oliv. to drought stress. Chinese Journal of Eco-Agriculture, 20(8):1059-1065.

Qiao G., Wen X. P., Yu L. F. and Ji X. B. 2011. The enhancement of drought tolerance for pigeon pea inoculated by arbuscular mycorrhizae fungi. Plant, Soil and Environment, 57 (12): 541-546.

Ratzinger A., Riediger N., von Tiedemann A. and P. Karlovsky. 2009. Salicylic acid and salicylic acid glucoside in xylem sap of Brassica napus infected with Verticillium longisporum. Journal of Plant Research, 122:571-579.

Reusche M., Thole K., Janz D., Truskina J., Rindfleisch S., Drübert C., Polle A., Lipka V. and Teichmann T. 2012. Verticillium infection triggers VASCULAR-RELATED NACDOMAIN7-dependent de novo xylem formation and enhances drought tolerance in Arabidopsis. The Plant Cell, 24: 3823-3837. 
Reusche M., Truskina J., Thole K., Nagel L., Rindfleisch S., Tran Van T. and Braus-Stromeyer S. A. 2014. Infections with the vascular pathogens Verticillium longisporum and Verticillium dahliae induce distinct disease symptoms and differentially affect drought stress tolerance of Arabidopsis thaliana. Environmental and Experimental Botany, in press, Accepted manuscript.

Ringnér M. 2008. What is principal component analysis? Nature Biotechnology, 26(3):303-304.

Ristaino J. B. and Duniway J. M. 1989. Effect of preinoculation and postinoculation water stress on the severity of Phytophthora root rot in processing tomatoes. Plant disease, 73(4): 349-352.

Ruiz-Lozano J. M., Azcon R. and Gomez M. 1995. Effects of arbuscular-mycorrhizal Glomus species on drought tolerance: Physiological and nutritional plant responses. Applied and Environmental Microbiology, 61(2):456-460.

Rygulla W., Friedt W., Seyis F., Lühs W., Eynck C., von Tiedemann A. and R. J. Snowdon. 2007a. Combination of resistance to Verticillium longisporum from zero erucic acid Brassica oleracea and oilseed Brassica rapa genotypes in resynthesized rapeseed (Brassica napus) lines. Plant Breeding, 126:596-602.

Rygulla W., Snowdon R. J., Eynck C., Koopmann B., von Tiedemann A., Lühs W. and Friedt W. 2007b. Broadening the genetic basis of Verticillium longisporum resistance in Brassica napus by interspecific hybridization. Phytopathology, 97:1391-1396.

Saadia M., Jamil A., Akram N. A. and M. Ashraf. 2012. A study of proline metabolism in canola (Brassica napus L.) seedlings under salt stress. Molecules, 17: 5803-5815.

Sadaqat H. A., Tahir M. H. N. and Hussain M. T. 2003. Physiogenetic aspects of drought tolerance in canola (Brassica napus). International Journal of Agriculture and Biology, 5(4):611-614.

Sadras V. O., Quiroz F., Echarte L., Escande A. and Pereyra V. R. 2000. Effect of Verticillium dahliae on photosynthesis, leaf expansion and senescence of field-grown sunflower. Annals of Botany, 86: 1007-1015.

Sánchez-Blanco M. J., Ferrández T., Morales M. A., Morte A. and Alarcón J. J. 2004. Variations in water status, gas exchange, and growth in Rosmarinus officinalis plants infected with Glomus deserticola under drought conditions. Journal of Plant Physiology, 161:675-682.

Sangtarash M. H., Qaderi M. M., Chinnappa C. C. and Reid D. M. 2009. Differential sensitivity of canola (Brassica napus) seedlings to ultraviolet-B radiation, water stress and abscisic acid. Environmental and Experimental Botany, 66:212-219.

Savitch L. V., Allard G., Seki M., Robert L. S., Tinker N. A., Huner N. P. A., Shinozaki K. and Singh J. 2005. The effect of overexpression of two Brassica CBF/DREB1-like transcription factors on 
photosynthetic capacity and freezing tolerance in Brassica napus. Plant and Cell Physiology, 46(9):1525-1539.

Senthil-Kumar M. and Mysore K. S. 2012. Ornithine-delta-aminotransferase and proline dehydrogenase genes play a role in non-host disease resistance by regulating pyrroline-5carboxylatemetabolism-induced hypersensitive response. Plant, Cell and Environment, 35:13291343.

Sepehri A. and Golparvar A. R. 2011. The effect of drought stress on water relations, chlorophyll content and leaf area in canola cultivars (Brassica napus L.). Electronic Journal of Biology, 7(3): 49-53.

Shirani Rad A. H. and Zandi P. 2012. The effect of drought stress on qualitative and quantitative traits of spring rapeseed (Brassica napus L.) cultivars. Žemdirbystè-Agriculture, 99(1):47-54.

Siddiqui Z. S., Khan M. A., Kim B. G., Huang J. S. and Kwon T. R. 2008. Physiological responses of Brassica napus genotypes to combined drought and salt stress. Plant Stress, 2 (1):78-83.

Silvente S., Sobolev A. P. and Lara M. 2012. Metabolite adjustments in drought tolerant and sensitive soybean genotypes in response to water stress. PLoS ONE, 7(6): e38554.

Sinaki J. M., ESlam M. H., Shirani Rad A. H., Noormohammadi G. and Tarei G. 2007. The effects of water deficit during growth stages of canola (Brassica napus L.). American-Eurasian Journal of Agricultural \& Environmental Science, 2(4):417-422.

Singh M., Chauhan J. S. and Meena S. S. 2009. Drought induced changes in water use efficiency and other morpho-physiological characters in Indian mustard (Brassica juncea L.). $16^{\text {th }}$ Australian Research Assembly on Brassicas, Ballarat Victoria, Australia.

Somasegaran, P. and H.J. Hoben. 1985. Methods in legume-rhizobium technology. Nitrogen fixation in tropical agricultural legumes and microbiological resources center (NifTAL), Paia, Hawaii.

Stanke C., Kerac M., Prudhomme C., Medlock J. and Murray V. 2013. Health effects of drought: a systematic review of the evidence. PLOS Currents Disasters. Edition 1.

Steventon L. A., Okori P. and Dixelius C. 2001. An investigation of the susceptibility of Arabidopsis thaliana to isolates of two species of Verticillium. Journal of Phytopathology, 149:395-401.

Sundaresan S. and P. Sudhakaran R. 1995. Water stress-induced alterations in the proline metabolism of drought-susceptible and -tolerant cassava (Manihot esculenta) cultivars. Physiologia Plantarum, 94:635-642.

Szabados L. and Savouré A. 2010. Proline: a multifunctional amino acid. Trends in Plant Science, 15(2):89-97.

Tambussi E. A., Bort J. and J. Araus L. 2007. Water use efficiency in C3 cereals under Mediterranean conditions: a review of physiological aspects. Annals of Applied Biology, 150:307-321. 
Tang D., Qian H., Zhao L., Huang D. and Tang K. 2005. Transgenic tobacco plants expressing BoRS1 gene from Brassica oleracea var. acephala show enhanced tolerance to water stress. Journal of Biosciences, 30(5):647-655.

Thorne E. T., Stevenson J. F., Rost T. L., Labavitch J. M. and Matthews M. A. 2006. Pierce's disease symptoms: Comparison with symptoms of water deficit and the impact of water deficits. American Journal of Enology and Viticulture, 57:1-11.

Tisserat N. A. 2004. Canker diseases of trees. Kansas State University. October 2004, Kansas, USA.

Ullah F. Bano A. and Nosheen A.. 2012. Effects of plant growth regulators on growth and oil quality of canola (Brassica napus L.) under drought stress. Pakistan Journal of Botany, 44(6): 1873-1880.

Valliyodan B. and Nguyen H. T. 2006. Understanding regulatory networks and engineering for enhanced drought tolerance in plants. Current Opinion in Plant Biology, 9:189-195.

Wiese J, Kranz T. and Schubert S. 2004. Induction of pathogen resistance in barley by abiotic stress. Plant Biology, 6(5):529-36.

Xiang Y., Huang Y. and Xiong L. 2007. Characterization of stress-responsive CIPK genes in rice for stress tolerance improvement. Plant Physiology, 144:1416-1428.

Xu P., Chen F., Mannas J. P., Feldman T., Sumner L. W. and Roossinck M. J. 2008. Virus infection improves drought tolerance. New Phytologist, 180: 911-921.

Xue X., Liu A. and Hua X. 2009. Proline accumulation and transcriptional regulation of proline biothesynthesis and degradation in Brassica napus. Biochemistry and Molecular Biology Reports, 42(1):28-34

Yadeta K. A. and Thomma B. P. H. J. 2013. The xylem as battleground for plant hosts and vascular wilt pathogens. Frontiers in Plant Science, 4(97):1-12.

Yang S., Vanderbeld B., Wan J. and Huang Y. 2010. Narrowing down the targets: Towards successful genetic engineering of drought-tolerant crops. Molecular Plant, 3(3):469-490.

Yu S. W., Zhang L. D., Zuo K. J., Tang D. Q., Sun X. F. and Tang K. X. 2005. Brassica napus L. homeodomain leucine-zipper gene BnHB6 responds to abiotic and biotic stresses. Journal of Integrative Plant Biology, 47(10):1236-1248.

Zakirullah, Swati Z. A., Ahmad A. and Raziuddin. 2000. Morpho-physiological response of selected Brassica lines to moisture stress. Pakistan Journal of Biological Sciences, 3(1):130-132.

Zeise K. and von Tiedemann A. 2001. Morphological and physiological differentiation among vegetative compatibility groups of Verticillium dahliae in relation to $V$. longisporum. Journal of Phytopathology, 149:469-475. 
Zeise K. and von Tiedemann A. 2002. Host specialization among vegetative compatibility groups of Verticillium dahliae in relation to Verticillium longisporum. Journal of Phytopathology, 150, 112119.

Zeise, K. 1992. Gewächshaustest zur Resistenzprüfung von Winterraps (Brassica napus L. var. oleifera Metzger) gegen den Erreger der Rapswelke Verticillium dahliae Kleb. Nachrichtenblatt DeutscherPflanzenschutzdienst, 44:125-128.

Zentková I. and Cvengrošová F. 2013. The utilization of rapeseed for biofuels production in the EU. Visegrad Journal on Bioeconomy and Sustainable Development, 2(1)11-14.

Zhou L., Hu Q., Johansson A. and Dixelius C. 2006. Verticillium longisporum and V. dahliae: infection and disease in Brassica napus. Plant Pathology, 55:137-144.

Zhou M. L., Ma J. T., Pang J. F., Zhang Z. L., Tang Y. X. and Wu Y. M. 2010. Regulation of plant stress response by dehydration responsive element binding (DREB) transcription factors. African Journal of Biotechnology, 9(54):9255-9279. 


\section{General discussion}

Since 2000, the global OSR production has surpassed cottonseed and became the world's second most important source of vegetable oil (Carré and Pouzet, 2014). The recently originated vascular pathogen of OSR, V. longisporum (Inderbitzin et al., 2011a), is becoming a potential threat to OSR production particularly in the northern European countries where this crop has become the major oilseed due to the expansion of biodiesel industry (Carré and Pouzet, 2014). Under conditions of high disease severity, V. longisporum can cause severe yield losses in OSR (Dunker et al., 2008; Gladders, 2009) and its recent report from UK and Czech Republic (CABI and EPPO, 2011) may also suggest its great potential for rapid expansion to new geographic regions. As no other management options, other than the use of plant resistance, are effective or available against this pathogen, the efforts on exploration of control options so far were mainly focusing on identification or development of OSR varieties with enhanced resistance. The present study was one of the efforts towards improving the resistance of OSR against VL with a particular focus on understanding mechanisms of disease resistance and investigation of the physiological and agronomic significance of VL resistance mechanisms under drought stress conditions. The work encompassed a range of studies from identification of resistant genotypes (Chapter 2) towards searching (Chapter 3) and functional analysis (Chapter 4) of VL- resistance factors in winter OSR.

\subsection{Identification of $B$. napus genotypes with enhanced resistance to $V$. longisporum}

With a general objective of identifying VL-resistant winter OSR genotypes, screening experiments were conducted under different experimental condition, namely in a climate controlled greenhouse, under field condition with different edaphic, climatic and disease pressure conditions, and under outdoor condition which represent an intermediate situation between the greenhouse and field experiments.

In the greenhouse experiments, the resistance response of more than 230 winter OSR genotypes (DH lines and other accessions) was evaluated using an efficient screening method (Eynck, 2008) that involves assessment of disease severity and stunting effect. From the analysis of the disease severity parameter NNA, it was found that more than $38 \%$ of the tested lines had less disease severity than the resistant standard Express (NNA=0.58). On the other hand, only very few lines (3\%) showed severe susceptibility with greater NNA values than the susceptible standard Falcon (NNA=1.42). For the remaining larger proportion (58\%) of the tested lines, a wide range of disease severity, ranging between the NNA value of the susceptible and resistant standards, was observed (Appendix 2.3). With few exceptions, comparable results (with more or less similar proportion compared to the one observed in disease severity results) were obtained from assessment of stunting effect (Appendix 2.3). The 
consistency of disease evaluation results obtained from stunting and disease index measurements were further verified by correlation analysis that showed significantly strong positive relationship of these parameters $(r=0.66)$, indicating that both parameters are most appropriate and reliable phenotypic traits for evaluation of VL resistance in oilseed rape under controlled conditions. Moreover, since greenhouse screening involves the use of non-vernalized few weeks old seedlings, this method allows the evaluation of quite a large number of plants within a relatively small space and short period. In general, since the genetic background of the tested genotypes is confidential, it was not possible to explain the possible source and mechanisms of resistance in this set of lines. Nevertheless, the present greenhouse disease screening results obtained from a large number of plant materials not only showed the availability of VL-resistance in Brassica species, but also demonstrated the presence of a wide range of variations in level of resistance. This is in strong agreement with previous reports that showed the presence of various levels of VL-resistance in different species of the Brassica genus (Rygulla et al., 2007a; Rygulla et al., 2007b; Eynck et al., 2009a; Happstadius et al., 2003). In conclusion, of the 235 B. napus accessions screened under greenhouse conditions, 23 genotypes that showed very strong resistance (with NNA and NRS values less than 0.40; Appendix 2.3) are recommendable for further evaluation under field conditions.

Field experiments were conducted in three locations and for three consecutive seasons (2010/11, 2011/12 and 2012/13). During each season trial, 40 genotypes were evaluated. Of these, fourteen best performing lines (See section 2.4.2.1) were selected based on previous greenhouse screening results. These lines and the four reference varieties were evaluated in all season trials across all locations. The remaining 22 lines were also evaluated in all locations but only for one season (See section 2.4.2.2). The results obtained from both sets of lines showed the significant influence of genotype, seasonal variations and locational differences in disease development. The following discussions based on the results of the first set of 18 genotypes (13 DH lines, 1 accession and 4 reference varieties) which reasonably reflect the interaction effects among the aforementioned three factors.

Disease assessment under field conditions was performed using two methods, disease index scoring (SDI and RDI) and quantification of fungal DNA by qPCR. Due to the loss of plants by hard winter in 2010/11 and 2011/12 in Göttingen and in 2012/13 in Svalöv, disease index data from these trials were unavailable. In Fehmarn, disease index data was recorded from all season trials. For the rest six different trials conducted in different locations or seasons, analysis of variance showed that except in 2010/11 in Fehmarn where SDI showed significant differences among the reference varieties and some DH lines 
(Table 2.3), both disease index measurements showed no significant difference among either the reference standards or the DH lines. Similarly, none of the disease index measurements in Svalöv showed significant differences among any of the genotypes (Table 2.4). In Göttingen, the only disease index data obtained from 2012/13 also showed a similar trend. Here, with the exception of Oase and SW 08-190002-8 that had significantly lower disease index compared to the susceptible standard Laser, no significant difference was found among the rest of the $13 \mathrm{DH}$ lines and the remaining 3 reference varieties (Table 2.5).

Table 5.1 Seasonal and locational comparisons of Verticillium longisporum disease index measured from stubbles of 18 B. napus genotypes at $\mathrm{BBCH}$ growth stage 97 (after grain harvest).

\begin{tabular}{lcccccc} 
& \multicolumn{7}{c}{ Growing season } \\
Location & $2010 / 11$ & \multicolumn{2}{c}{$2011 / 12$} & $2012 / 13$ \\
& SDI & RDI & SDI & RDI & SDI & RDI \\
\hline Fehmarn & 2.73 & 1.67 & 2.16 & 2.14 & 1.95 & 2.11 \\
Svalöv & 2.52 & 2.42 & 1.44 & 1.56 & ND & ND \\
Göttingen & ND & ND & ND & ND & 1.17 & 1.18
\end{tabular}

Data shown are average disease index values of 18 genotypes (13 DH lines, 1 accession and 4 reference varieties). Disease index was measured using a key with 1-4 scales (Figure 2.1). SDI, shoot disease index. RDI, root disease index. ND, no data due to loss of plants by hard winter.

Regarding comparison of disease index across locations, except RDI in 2010/11, during the first two seasons (2010/11 and 2011/12), there was relatively lower disease severity in Svalöv than Fehmarn (Table 5.1). In 2012/13, compared to Göttingen where the trial was conducted using artificial inoculation, again relatively higher disease severity was measured in Fehmarn. Comparison of disease index across years was done for Fehmarn and Svalöv trials where data were available at least from two seasons. Accordingly, again with the exception of Fehmarn RDI, in 2010/11, compared to the other two years, relatively higher disease index in both locations was recorded in 2010/11 (Table 5.1). In Göttingen, since the trials in 2010/11 and 2011/12 were completely destroyed by hard winter, comparison of infestation across years was not possible (Table 5.1). In general, since there was no qPCR data in 2010/11 from where relatively high disease index was recorded in both Fehmarn and Svalöv, it was not possible to verify phenotypic disease evaluation results with molecular disease assessment. Nevertheless, the slightly lower average temperatures and higher moisture conditions in both locations might be among the possible factors that could explain the slightly increased disease index recordings. Further correlation analysis across locations showed no significant relationship among any of the disease index results (Table 2.7). Within the same location however, some significant correlations between SDI 
and RDI and between disease index and greenhouse/outdoor results were found (Table 2.6). The presence of high natural VL infestation in Svalöv and Fehmarn and little/no infestation in Göttingen is already known from previous studies (Knüffer, 2013; Eynck, 2008; Johansson et al., 2006). Thus, in addition to the differences in amounts of inoculum in the soil and variations in climatic and edaphic factors (Table 2.2), possible locational differences in terms of composition and diversity of virulent $\mathrm{VL}$ isolates might explain the variations in disease levels across locations.

Regarding evaluation of disease severity by molecular (qPCR) method, assessment was done in 2011/12 and 2012/13. In this report, qPCR data from Fehmarn and Svalöv in 2011/12 and from Göttingen in 2012/13 were included. For the remaining experiments, qPCR data were not available either due to the loss of plants by the hard winter or because sample analysis is not finalized yet. Unlike the disease index results however, qPCR results revealed significant differences not only among the reference standards but also among the DH lines. In Fehmarn for example, in 2011/12, the amount of VL DNA quantified from the susceptible cultivars Falcon and Laser was 9.8 and $11.4 \mathrm{ng} / \mathrm{gram}$ of stem dry matter, respectively. From the resistant genotypes Express and Oase, significantly lower amounts, 0.8 and $1.8 \mathrm{ng} / \mathrm{g}$, respectively, were quantified. Furthermore, significant differences among the DH lines were observed. The least quantity of fungal DNA $(0.13 \mathrm{ng} / \mathrm{g})$ which is significantly lower than both resistant controls was detected from accession SEM 05-500256. It is so interesting that this genotype, which is used as a resistant reference in the other greenhouse studies focusing on resistance mechanisms (chapter 3 and 4), showed quite remarkable resistance under field conditions (Table 2.3). During the same season (2011/12), qPCR results in Svalöv also showed a similar trend. Compared to the resistant standards, about two fold higher fungal DNA was quantified from susceptible standards. However, this difference was not statistically significant, possibly due to the high variability between replicates. Regarding the DH lines, seven had significantly lower amounts of VL DNA compared to the susceptible controls. Of these lines, three of them including SEM05-500256 had significantly lower VL DNA than both resistant controls (Table 2.4). As observed in previous year experiments in Fehmarn and Svalöv, the qPCR data in 2012/13 in Göttingen also revealed significant difference among genotypes. Accordingly, the highest quantity of VL DNA was recorded for the susceptible standard Laser (10.4ng/g) flowed by the other susceptible standard Falcon $(1.5 \mathrm{ng} / \mathrm{g})$. These quantities were significantly different from the amounts detected in the resistance standards Oase $(0.37 \mathrm{ng} / \mathrm{g})$ and Express $(0.08 \mathrm{ng} / \mathrm{g})$. Regarding the remaining DH lines, eight of them had significantly lower VL DNA than both susceptible standards. In this location as well, genotype SEM05-500256, from which the third least amount of fungal DNA (0.12ng/g) was quantified, displayed very high level of resistance response (Table 2.5). As is the case of disease 
index assessment, since there were no complete data sets from the different season trials of the same location available, comparison of disease levels across years by qPCR data was not possible. With regard to variations across locations however, it was found that unlike in Göttingen (where the experiment was conducted with artificial application of additional inoculum), disease pressure was significantly higher in Svalöv and Fehmarn (Table 5.2). This is in strong agreement with the results of disease index evaluations. Regarding correlation of results, it was so interesting that qPCR was the only field disease evaluation parameter that showed very strong positive correlation ( $r=0.86)$ across locations (Table 2.7). Furthermore, unlike disease index measurements, qPCR results were more significantly correlated to field disease index recordings in different locations and years. It also showed significant correlations with NNA and NRS results of greenhouse and outdoor experiments (Table 2.6). Overall, based on the results of the present multi-site field trial conducted for three seasons, the following winter OSR lines SEM05-500256, SW08-190001-12, SW08-190001-7, SW08-190001-25, SW08-190002-9, SW08-190002-8 and SW08-190002-11 are highly recommended for use as parent materials in future breeding programs that aim at integration of Verticillium longisporum resistance traits into elite winter oilseed rape varieties.

Table 5.2 Comparisons of Verticillium longisporum disease severity across seasons and locations at $\mathrm{BBCH}$ growth stage 80 .

\begin{tabular}{lccc}
\hline \multirow{3}{*}{ Location } & \multicolumn{3}{c}{ VL DNA in dry stem tissue (ng/g) } \\
& $2010 / 11$ & $2011 / 12$ & $2012 / 13$ \\
\hline Fehmarn & ND & 2.23 & ND \\
Svalöv & ND & 2.35 & ND \\
Göttingen & ND & ND & 0.91 \\
\hline
\end{tabular}

Data shown is average VL DNA quantified from 18 genotypes (13 DH lines, 1 accession and 4 reference varieties) by qPCR. VL, Verticillium longisporum. ND, no data either due to loss of plants by hard winter or because sample analysis is not yet completed.

The locational variation in disease development shown in the above experiments was verified in another parallel experiment that involved qPCR analysis of disease severity in field grown different aged reference varieties. This experiment was conducted in 2012/13 in all the three locations and disease assessment by qPCR was done at three different growth stages, 50\% flowering (BBCH65), fruit development $(\mathrm{BBCH} 75)$ and ripening $(\mathrm{BBCH} 80)$. The core objective of this study was to identify the critical crop growth stage for the detection of VL-infection and further differentiation of resistant and susceptible genotypes. Furthermore, the applicability of this method for general assessment of disease epidemics was validated. 
Results showed that regardless of disease resistance, detection of the VL in OSR stem tissues was possible as early as $\mathrm{BBCH} 65$. However, distinct separation of resistance and susceptible varieties and differentiation of infestation levels across locations was achieved at $\mathrm{BBCH} 80$; verifying the long biotrophic latent phase of VL disease cycle in OSR in the field (Dunker et al., 2008; Gladders, 2009). Göttingen was the site where the least level of infestation was observed (Fig. 2.6A). In this location, the average VL DNA quantified from cultivars Oase, Express, Laser and Falcon were 0.083, 0.37, 1.47 and $10.4 \mathrm{ng} / \mathrm{g}$, respectively. Interestingly, even under this very low infestation level, the amount of VL DNA quantified from the susceptible varieties was generally higher than the resistance genotypes. The second highest level of infestation was recorded in Fehmarn. Here, compared to resistant standards, high amounts of VL DNA were detected from the susceptible varieties with the highest quantity which is significantly higher than all the remaining three reference standards $(79.9 \mathrm{ng} / \mathrm{g})$ was detected from cultivar Falcon (Fig. 2.6B). This quantity is almost 8 fold higher compared to the highest amount in Göttingen. Again considering qPCR data, compared to all locations, the highest infestation was found in Svalöv (Fig. 2.6C). Here, compared to the resistant cultivars, more than 300 fold VL DNA was quantified from the susceptible varieties. The highest significant infestation $(3,252.6 \mathrm{ng} / \mathrm{g})$ was found in Laser and this amount was 141 and 314 fold higher compared to the infestation in Fehmarn and Göttingen, respectively. Similar trend was observed on the other susceptible cultivar Falcon where infestation in Svalöv was 7 and 377 fold higher compared to Fehmarn and Göttingen, respectively. The observed locational variations were not only in terms of infestation levels. It was also noticed that, unlike in Fehmarn and Göttingen, results from Svalöv were poorly correlated with each other and with greenhouse and outdoor results as well. And this is in strong agreement with the correlation analysis results of disease index measurements (Table 2.6). Furthermore, when the two susceptible genotypes were compared, the highest VL DNA in Svalöv and Göttingen was found in Laser. In Fehmarn however, the highest infestation occurred in Falcon. As mentioned earlier, the possible reasons for locational variation in terms of levels of disease infestation and degree of correlation between field trials and experiments in controlled conditions could possibly be due to the climatic, edaphic and pathogen factors that potentially influence host-pathogen interaction and thereby host resistance or pathogen aggressiveness (Agrios, 1995). The above described variations in VL disease infestations across locations was comparable with disease index assessment results of previous studies conducted with other sets of OSR genotypes (Eynck, 2008; Knüfer, 2013). On the other hand, it somehow contradicts with the 2010/11 and 2011/12 disease index results of the present study that showed relatively high levels of infestation in Fehmarn (Table 5.1). 
In general, the results of the present study demonstrated that with the sole use of phenotypic methods (SDI and RDI), it is not always possible to differentiate field performance of resistance and susceptible genotypes. Besides, results obtained from this method show high variability across different locations and seasons. Differences in growth habits among cultivar (late vs late ripening) also significantly determine microsclerotia development and thus disease index (Knüfer, 2013). In addition, in most cases, stubble disease index results were hardly and only occasionally correlated with field qPCR as well as with NNA and NRS results of greenhouse and outdoor experiments. Therefore, as this parameter is influenced by many factors, decisions on OSR resistance to $V$. longisporum based on field disease index should either be considered with caution or should be supported with additional evidence such as molecular data. Unlike the field phenotypic parameters (SDI and RDI) however, greenhouse and outdoor phenotypic disease evaluation parameters (NNA and NRS) distinctly differentiated resistant and susceptible genotypes (Figure 2.3; Figure 2.8). This could partly be because of the possibility of controlling inoculum and climatic conditions in these experiments. Compared to NRS however, NNA was more correlated with field data (Table 2.6A). This is somehow suggesting the effects of genotype on the extent of VL-induced stunting which is in strong agreement with the observations in greenhouse screening experiments where some genotypes with resistant reactions in AUDPC measurements showed severe stunting (Appendix 2.3).

The other important issue regarding stubble disease index is the time point at which assessment is carried out. After all, understanding disease threshold at or after crop maturity stage (after grain harvest) is not useful, particularly with regard to taking immediate control action. Because, at this time point, plants have already attained the maximum stage of development and therefore application of any possible control measure may not be economical. Nevertheless, this does not mean that the information obtained from this method is totally irrelevant. Because, determination of infestation levels on crop residue (stubble in this case) may still provide sufficient information which help to decide on the use of some control measures (such as management of crop residue) that help reduce the potential build-up of inoculum in the soil and thus future disease epidemics. Furthermore, knowledge on the extent of inoculum on crop residues provide valuable information on the amount of pathogen resting propagules (microsclerotia) joining the soil bank. Estimation of inoculum levels in the soil is one of the basic and most important inputs required for forecasting disease pressure and this information will help to plan future production schemes such as decision making in crop rotation programs. 
On the other hand, the use of qPCR for the evaluation of disease severity or VL resistance in OSR under field conditions seems to have several merits. First of all, with this method it is possible to detect even a very slight level of infection (up to few pictograms of fungal DNA per gram of stem material) and at a relatively early growth stage (at least at $\mathrm{BBCH} 65$ ). Later at crop maturity (BBCH75-80), the method allows distinct differentiation of resistant and susceptible plants. More importantly, qPCR results are not only significantly correlated across locations and seasons but also are more consistent with the results of the experiments in controlled environments (greenhouse and outdoor) and occasionally with field disease index evaluations as well. In conclusion, quantification of $V$. longisporum by qPCR seems to be the most consistent, fast and possibly cheaper alternative to survey VL disease severity or evaluate resistance of OSR genotypes in the field.

In general, it can be concluded that with the currently available sampling techniques, primers and PCR conditions, reliable and consistent assessment of VL disease severity in the field is mainly possible by qPCR and particularly when the crop is approaching the ripening stage (BBCH 75-80). Development of a method that enables early (in autumn or spring) assessment of infection or disease severity by qPCR from root or hypocotyl tissue may provide a better and timely information about disease epidemics or level of plant resistance. Therefore, in order to reduce quantitative or qualitative yield losses that might be caused due to the late recognition of $V$. longisporum epidemics in OSR, development of a more elegant method (for example monitoring disease severity in roots during early stages of crop growth or development of a model that enables early detection or forecast of disease severity), should be the focus of future research.

\subsection{Searching for VL resistance factors in OSR xylem sap}

Understanding mechanisms of VL-resistance was one of the major objectives of the present study. To this end, the investigations on xylem sap residing cultivar-related $V$. longisporum resistance factors in oilseed rape were undertaken in different experiments involving greenhouse studies, in vitro bioassays and biochemical analyses. Three B. napus genotypes with differential resistance towards VL were used. Greenhouse studies were conducted for the purpose of verifying resistance responses of the genotypes and collection of xylem sap. In vitro bioassays and biochemical analyses were performed to study the effects of OSR xylem sap constituents on VL growth.

Analysis of the disease evaluation data obtained from assessment of disease index and quantification of VL DNA (Fig. 3.2) showed the development of very slight symptoms or disease level in genotype Aviso and SEM, confirming their resistance to VL. On the other hand, the very fast and high level of disease 
development in cultivar Falcon demonstrated its high susceptibility to VL. Further evidences for the consistent response of the three genotypes to VL-infection was shown in agronomic traits where unlike SEM, significantly higher reduction of plant height, stem thickness and shoot dry mater accumulation due to infection occurred in cultivar Falcon (Figure 3.3). Correlation analysis also showed very strong, significant, positive correlation within disease or agronomic parameters and negative correlations between disease and agronomic parameters (Table 3.6). VL-resistance of genotype SEM (SEM 05500256) and susceptibility of Falcon under different experimental conditions (greenhouse, outdoor and field) was also shown in another independent disease screening experiments (Chapter 2). Furthermore, similar results from previously field and greenhouse disease screening studies have been reported (Knüfer, 2013; Eynck et al., 2009b; Rygulla et al, 2007b). Greenhouse and field studied by Keunecke (2009) also demonstrated the resistance of cultivar AVISO to VL.

From the phenotypic and molecular greenhouse disease evaluation results described in Chapter 2 and Chapter 4 and from previous studies on mechanisms of VL resistance in OSR (Eynck et al., 2009b; Obermeier et al., 2013), it is well known that 21-28 DPI is the critical time point for resistance and susceptible genotypes to show marked differences in disease symptoms. That means, the level of accumulation of resistant factors in resistant genotypes at this time point is sufficient to reduce or restrict further growth and development of the pathogen. Hence, in order to compare the nature of VL growth in xylem sap of resistant (SEM) and susceptible (Falcon) plants, xylem sap derived from different aged plants was used in in vitro bioassay. Assessment of xylem sap fungal growth by spectrophotometry revealed that regardless of plant genotype, xylem sap served as a suitable medium for the growth of VL. Initiation of spore germination and further fungal growth was detectable 2-3 days after incubation. Later 5 days after incubation, robust fungal growth that covered the whole area of the microtitre plate wells was observed. Compared to its typical slow growth on artificial medium (such as PDA) which usually takes several weeks to cover a full radius of a $90 \mathrm{~mm}$ Petri dish (Fig. 1.2), VL growth on xylem sap seems a bit faster. Further inspection of fungal growth even one week after incubation shows a similar story, no significant growth difference in xylem sap derived from plants with contrasting response to VL infection. Similarly, comparisons of fungal growth in xylem sap of mock and VL-inoculated plants also revealed the absence of infection induced VL-resistance factor contributing to significant reduction of in vitro VL growth. Nevertheless, whether xylem sap constitutes of resistant and susceptible plants differentially affect fungal sporulation, was not investigated here. 
Interestingly, fungal growth on filtered xylem sap was slightly reduced, but did not significantly affect fungal growth. The minor protein binding property of the syringe filter used to prepare filtered xylem sap treatments might adsorb some substances that are required by the fungus and this might cause the slight reduction of fungal growth. Results of protein assay also showed slightly reduced amounts of total soluble proteins in filtered samples. Again, the difference in concentration of total soluble proteins in xylem sap of resistant and susceptible genotypes was not significant (Table 3.3). Even the infectioninduced slight increase in protein concentration occurred similarly in both susceptible and resistant genotypes. Similar results have been reported in tomato where infection with $V$. albo-atrum caused a general increase of xylem sap proteins irrespective of plant resistance to the disease (Dixon and Pegg, 1972). The above mentioned facts are in strong agreement with the present bioassay results where no significant effect of plant genotype, VL-infection and filtration of xylem sap on in vitro growth of VL is shown. Unfortunately, compared to other plant fluids or tissue extracts, very little is known about the composition and role of xylem sap constituents in plants in general and in B. napus in particular. In agreement with the findings of the present study, the work by Floerl el al. (2008) showed no effect of VL-infection on $B$. napus total xylem sap protein concentration. This study further demonstrated $B$. napus xylem sap as a suitable medium for the growth of VL. However, in contrast to this work, the study used a single susceptible genotype and found significant reduction of in vitro growth of VL on xylem sap of infected plants. As it is shown in another pathosystem, several factors such as symbiotic or pathogenic interactions can determine the level and composition of xylem sap proteins and other constitutes (Subramanian et al, 2009).

The other xylem sap constitutes related to plant defence are plant hormones. The role of the wellknown defence hormone SA in B. napus-VL interaction was investigated in previous studies. Results provided a strong evidence for the lack of correlation between the enhanced accumulation of SA in xylem sap or tissue extracts and cultivar-related resistance of OSR against VL. These studies rather demonstrated VL-infection induced increased accumulation of SA in susceptible cultivars than in resistant plants (Ratzinger et al., 2009; Siebold, 2012; Kamble et al., 2013). Similarly, in V. dahliaeArabidopsis interaction, impairment of SA does not cause either high sensitivity to disease or any change in symptom development (Veronese et al., 2003). Other plant hormones such as JA and ABA seems to have no or insignificant role in Arabidopsis- and B. napus- VL interaction (Ratzinger et al., 2009; Veronese et al., 2003). 
The other interesting observation of the present study that exactly correlates with natural (field) lifecycle of the pathogen is its enhanced in vitro growth on xylem sap of older aged plants (Fig. 3.8). As thoroughly discussed in previous chapters (Chapter 1 and 2) and section 5.1 of this chapter as well, VL shows a long biothrophic latency period during early stages of plant development. Late in the growing season, the pathogen undergoes fast development and rapidly disseminate to the above-hypocotyl shoot part, leading to the development of the typical phenotypic symptoms (Knüfer, 2013). It is possible that VL can sense signals of plant developmental stages present in OSR xylem sap. This may possibly explain why the fungus displayed fast in vitro growth in xylem sap of older plants. The increased accumulation xylem sap sugars in older plants which is also known for other crop species such as tomato (Wang and Bergeson, 1974)and Arabidopsis (Yu et al., 2013) could also be a possible explanation for enhanced growth of VL in xylem sap of older aged plants.

This is a first study on functional analysis of cultivar related VL-resistance factors in winter OSR xylem sap. It provided concrete evidence that OSR xylem sap, irrespective of plant genotype, provide a suitable nutritional and chemical environment for the growth of VL. The slightly increased total soluble protein and sugar content in xylem sap of infected plants also demonstrates possible VL-induced changes in the composition or level of OSR xylem sap constitutes. However, since these quantitative changes were not significantly different between resistant and susceptible genotypes, it can be concluded that OSR soluble xylem sap constituents are not playing a role as major resistance factors for cultivar-related winter oilseed rape resistance against VL. This is in strong agreement with the findings of previous studies on mechanisms of VL-resistance in OSR that demonstrated the significant role of cell wall bound metabolites and physical barriers in resistance of OSR to V. longisporum (Eynck et al., 2009b; Obermeier et al., 2013). Nevertheless, further studies that encompass a large number of genotypes and assessment of other parameters such as fungal sporulation are suggested.

\subsection{B. napus - V. longisporum interaction under drought stress conditions}

The main abiotic stress factor affecting plant production particularly under the changing global climate is drought stress. Understanding the extent of disease development and the nature of plant resistance under abiotic stress factors is therefore indispensable. An extensive study that looked at the impact of VL-infection on drought stress resistance of OSR and also the influence of drought stress on B. napus $V L$ interaction was conducted. Here a brief discussion of the results is provided. Interested reader on the detailed results and discussions of this topic is advised to see section 4.4 of Chapter 4 . 
It is well known that, among other factors, vascular occlusions are one of the mechanisms contributing to VL resistance in OSR. Occlusions induced by wilt causing vascular pathogens have similar effect as different abiotic stress factors such as drought. In the present study, analysis of physiological processes, gene expression, and agronomic and biomass yield accumulation in VL-infected OSR plants revealed that infection induced changes in the vascular system (such as accumulation of occlusions) have no significant effect on plants response to drought stress. Except for slight changes observed in a few cases (see section 4.3), neither VL alone nor its interaction with drought or the genotype had a significant effect on the response of OSR to drought stress. Similar results from previous studies also showed no effects of VL infection in gas exchange of B. napus (Floerl et al., 2008) and Arabidopsis (Floerl et al., 2010). The presence of occlusion free vessels in VL-infected B. napus plants (Eynck et al., 2007) might provide sufficient room for the transport of adequate amount of water and mineral nutrients. This may possibly explain why most physiological processes are not significantly affected by infection and the resulting blockage of vessels by occlusions. More importantly, this could also be one of the reasons why VL infection in B. napus is not causing wilting symptom (Eynck et al., 2007).

On the other hand, as expected, drought stress was the major factor that caused substantial changes in all physiological parameters and expression of drought induced genes considered in this study. These effects were also significant irrespective of genotype and pathogen infection. Drought-induced significant reduction in gas exchange (Naderikharaji et al., 2008; Sangtarash et al., 2009) and RWC (Ullah et al., 2012; Khalili et al., 2012) is well documented in previous studies in B. napus and other crop species. Furthermore, drought-induced increased accumulation of proline and expression of drought inducible genes is reported in several previous studies (Ghaffari et al., 2011; Omidi, 2010; Kagale et al., 2007; Saadia et al., 2012; Xue et al., 2009).

Other factors responsible for the variation in drought-induced changes in plant physiology were genotype and plant part. In general, the VL-susceptible cultivar Falcon was less sensitive to drought than the resistant genotype SEM. Comparisons of drought induced genes expression also suggest that the accumulation of P5SC1, DREB2 and CIPK1 gene transcripts were relatively higher in Falcon than in SEM. This is in agreement with physiological and yield assessment results that showed better performance of Falcon under drought conditions. Nevertheless, since only two genotypes are analyzed in the present study (which does not represent the genetic diversity in B. napus), it should not be generalized that OSR genotypes with resistance to $\mathrm{VL}$ are always sensitive to drought stress. 
The other interesting observation regarding physiological parameters was the difference in physiological changes between mock- and VL-inoculated plants. Comparison of drought induced changes in gas exchange, hypocotyl proline content and WUE under mock- and VL-inoculation conditions indicated a slightly reduced impact of drought stress during infection with VL. The reduced impact of drought stress during infection regardless of plant resistance to the pathogen might be associated with the infection induced anatomical changes in the xylem tissue. As described in Eynck et al. (2007), colonization of xylem vessels with VL and accumulation of VL-induced vascular occlusions in B. napus are restricted to individual vessels and other adjacent vessels remain completely uninfected and free of obstructions. In general, it is known that plants with smaller number of pit pores or smaller vessel diameter are well adapted to environments prone to frequent soil water deficit. This is because such plants extract water at a lower rate and as a result they will not run out of water quickly. In B. napus-OSR pathosystem, unlike control plants with large number of occlusion free vessels, the reduced number of occlusion free vessels in VL-infected plants might possibly helped plants to easily transport the very small amount of water present in soil and also not to suffer from cavitation that usually occur at high water potentials. The slightly reduced expression of the drought inducible genes (HB6, P5SC1, DREB2, and P5SC2) in VLinfected treatments of at least for one of the genotypes might also suggest a reduced impact of drought stress during infection with VL. Yadeta and Thomma et al. (2013) also suggested that if less numbers of vessels are closed by occlusions, the host plant will not suffer from drought stress. Furthermore, a recent study in Arabidopsis has shown enhanced drought tolerance of VL-infected plants due to infection induced de novo xylem formation (Reusche et al., 2014). In another pathosystem, Xu et al. (2008) showed viral infection induced increase in osmoprotectant and antioxidant substances which provided better drought tolerance to several crop species. Enhanced mycorrhizal induced drought tolerance due to increased accumulation of osmolites and sugars, nutrient uptake and root growth etc. has been reported in different crop families including vegetables, cereals and legumes.

Regarding the impact of drought stress on disease development, the consistent responses of resistant and susceptible genotypes as measured by AUDPC, relative stunting and fungal DNA quantification measurements; and the very strong correlation of these parameters at all watering regimes (see Chapter 2 and 3) verified the stable response of plants to VL-infection regardless of the amount of water supply. This is a strong evidence for stable functioning of the quantitative VL-resistance mechanisms known in OSR even under conditions of severe drought conditions. Likewise, the high disease severity observed in the susceptible cultivar which was even significantly higher at severe drought stress suggests more 
vulnerability of susceptible plants to the disease under conditions of additional stresses, drought in this case.

Concerning agronomic and yield traits, the effect of both stress factors was obvious. While VL-infection caused significant reduction of plant height and stem thickness, it induced increased production of excessive branches. The effect of VL on these parameters was evident regardless of water supply and plant genotype; but, compared to the resistant genotype, the magnitude of the effect was significantly greater on the susceptible cultivar. Furthermore, in agreement to the physiological data, slightly lower effect of infection on plant height, stem thickness and branching was observed under severe drought condition. Unfortunately and partly because of their short life span, the abnormal excessive branches induced by VL did not result in increased biomass yield. In contrast to field conditions (Dunker et al., 2008; Gladders, 2009), symptoms of stunting, excessive branching and reduced stem thickness were only observed under greenhouse (Chapter 3 ) and outdoor (Chapter 2) conditions. Apart from climatic factors, the source, amount and method of inoculations (Chapter 2) might also contribute for the exceptional development of these symptoms in controlled environments. Similar to VL, drought stress also significantly reduced plant height and stem thickness but its effect on branching particularly on resistant genotype was not significant. In general, the results from the above three agronomic parameters provided additional evidence for the absence of significant additive adverse effect of drought stress on VL-resistance of OSR.

The other agronomic trait considered in this study was plant growth rate. Interestingly, results were in line with the differential reactions of the two genotypes towards VL-infection and drought stress. The VL-resistant genotype SEM showed relatively faster growth and development. As it is known from Verticillium resistance mechanisms in other crop species (Bell and Stipanovic, 1978; Hutson and Smith, 1980), early and rapid accumulation of resistance factors might contributed to VL-resistance of genotype SEM.

Regarding biomass yield, as expected, both VL-infection and drought stress had significant effect. The severity of biomass yield reduction was also in agreement with the differential reactions of the two genotypes to drought stress and VL-infection. Furthermore, regardless of plant genotype, the extent of DM yield loss was directly proportional to the magnitude of drought stress. The reasonable SDM obtained from VL-infected fully and moderately irrigated resistant plants, no effect of VL-infection on SDM yield of severely stressed resistant plants, and again no impact of infection on RDM yield of the resistant genotype SEM are in agreement with disease evaluation results. This is a clear evidence for 
stable operation of VL-resistance mechanisms regardless of drought stress. Besides, the relatively low rate of pathogen induced yield reduction under severely-stressed tolerant and susceptible plants (Table 4.13), which also correlated with reduced impact of drought on most physiological parameters in infected plants and the disease development (AUDPC) as well imply that VL-drought interaction may favor the host plant.

In general, when the impact of the two stress factors on OSR is compared, the effect of VL was more important than drought stress. The deep and numerous root system and other typical drought tolerance associated traits of OSR such as leaf hair and waxes might provide B. napus the ability to tolerate drought stress and perform very well under water deficit situations. This is strongly supported by a recent study that showed increased root growth of $B$. napus cultivars with an increase in duration and intensity of drought stress (Ashraf et al., 2013). In conclusion, results of the present comprehensive study suggested that severe drought may not favor VL while at the same time VL-infection is possibly triggering changes in plant metabolism or architecture that provide plants with some level of tolerance against drought stress. However, this does not mean that simultaneous exposure of $B$. napus to drought stress and VL-infection has less impact than the independent effects of each stress factor. Because, despite the consistent resistance to VL under drought conditions and the better drought tolerance of VLinfected plants, compared to the effects of either VL or drought stress, simultaneous exposure of OSR to both stresses factors cause signifiacntly higher yield loss; indicating the very significance of VL epidemics during periods of drought stress. 


\subsection{References}

Agrios G. N. 2005. Plant pathology ( $5^{\text {th }}$ edition). Elsevier-Academic Press. San Diego, CA.

Ashraf M., Shahbaz M. and Ali Q. 2013. Drought-induced modulation in growth and mineral nutrients in canola (Brassica napus L.). Pakistan Journal of Botany, 45(1): 93-98.

Bell A. A. and Stipanovic R. D. 1978. Biochemistry of disease and pest resistance in cotton. Mycopathologia, 65:91-106.

CABI and EPPO, 2011. Verticillium longisporum. Distribution maps of plant diseases. Map no. 1117 (Edition 1). ISSN 0012-396X. CAB International, Wallingford, UK.

Carré P. and Pouzet A. 2014. Rapeseed market, worldwide and in Europe. Oilseeds and fats crops lipids, 21 (1) D102.

Dixon G. R. and Pegg G. F. 1972. Changes in amino-acid content of tomato xylem sap following infection with strains of Verticillium albo-atrum. Annals of Botany, 36:147-54.

Dunker S., Keunecke H., Steinbach P. and von Tiedemann A. 2008. Impact of Verticillium longisporum on yield and morphology of winter oilseed rape (Brassica napus) in relation to systemic spread in the plant. Journal of Phytopathology 156: 698-707.

Eynck C. 2008. Identification of resistance sources and characterization of resistance factors in Brassica species to Verticillium longisporum. PhD thesis. Georg-August-Universität Göttingen, Göttingen, Germany.

Eynck C., Koopmann B. and von Tiedemann A. 2009a. Identification of Brassica accessions with enhanced resistance to Verticillium longisporum under controlled and field conditions. Journal of Plant Diseases and Protection, 116(2), 63-72.

Eynck C., Koopmann B., Grunewaldt-Stoecker G., Karlovsky P. and von Tiedemann A. 2007. Differential interactions of Verticillium longisporum and V. dahliae with Brassica napus detected with molecular and histological techniques. European Journal of Plant Pathology, 118:259-274.

Eynck C., Koopmann B., Karlovsky P. and von Tiedemann A. 2009b. Internal resistance in winter oilseed rape inhibits systemic spread of the vascular pathogen Verticillium longisporum. Phytopathology, 99:802-811.

Floerl S, Druebert C, Aroud HI, Karlovsky P, Polle A. 2010. Disease symptoms and mineral nutrition in Arabidopsis thaliana in response to Verticillium longisporum VI43 infection. Journal of Plant Pathology, 92 (3), 693-700.

Floerl S., Druebert C., Majcherczyk A., Karlovsky P., Kües U. and Polle A. 2008. Defence reactions in the apoplastic proteome of oilseed rape (Brassica napus var. napus) attenuate Verticillium longisporum growth but not disease symptoms. BMC Plant Biology, 8:129. 
Ghaffari G., Toorchi M., Aharizad S. and Shakiba M. R. 2011. Evaluation of traits related to water deficit stress in winter rapeseed cultivars. Universal Journal of Environmental Research and Technology, 1(3):338-350.

Gladders P. 2009. Relevance of Verticillium wilt (Verticillium longisporum) in winter oilseed rape in the UK. HCGA Research Review, 72:1-39.

Happstadius I., Ljungberg A., Kristiansson B. and Dixelius C. 2003. Identification of Brassica oleracea germplasm with improved resistance to Verticillium wilt. Plant Breeding, 122:30-34.

Hutson R. A. and Smith I. M. 1980. Phytoalexins and tyloses in tomato cultivars infected with Fusarium oxysporum f.sp. lycopersici or Verlicillium albo-atrum. Physiological Plant Pathology, 17:245-257.

Inderbitzin P., Davis R. M., Bostock R. M. and Subbarao K. V. 2011a. The Ascomycete Verticillium longisporum is a hybrid and a plant pathogen with an expanded host range. PLOS ONE, 6(3): e18260.

Johansson A., Goud Jan-K. C. and Dixelius C. 2006. Plant host range of Verticillium longisporum and microsclerotia density in Swedish soils. European Journal of Plant Pathology, 114:139-149.

Kagale S., Divi U. K., Krochko J. E., Keller W. A. and P. Krishna. 2007. Brassinosteroid confers tolerance in Arabidopsis thaliana and Brassica napus to a range of abiotic stresses. Planta, 225:353-364.

Kamble A., Koopmann B. and von Tiedemann A. 2013. Induced resistance to Verticillium longisporum in Brassica napus by b-aminobutyric acid. Plant Pathology, 62:552-561.

Keunecke H.. 2009. Einfluss von Kohlfliegenbefall auf die Infektion und Schadwirkung von Verticillium longisporum und Phoma lingam an Raps. PhD thesis. Georg-August-Universität Göttingen, Göttingen, Germany.

Khalili M., Aboughadareh A. P., Naghavi M. R. and Talebzadeh S. J. 2012. Response of spring canola (Brassica napus L.) genotypes to water deficit stress. International Journal of Agriculture and Crop Sciences, 4 (21):1579-1586.

Knüfer J. 2013. Improvement of winter oilseed rape resistance to Verticillium longisporum - Assessment of field resistance and characterization of ultrastructural plant responses. PhD thesis. GeorgAugust-Universität Göttingen, Göttingen, Germany.

Naderikharaji R., Pakniyat H. and Biabani A. R. 2008. Effect of drought stress on photosynthetic rate of four rapeseed (Brassica napus) cultivars. Journal of Applied Sciences, 8(23): 4460-4463.

Obermeier C., Hossain M. A., Snowdon R., Knüfer J., von Tiedemann A. and Friedt W. 2013. Genetic analysis of phenylpropanoid metabolites associated with resistance against Verticillium longisporum in Brassica napus. Molecular Breeding, 31:347-361. 
Omidi H., 2010. Changes of proline content and activity of antioxidative enzymes in two canola genotype under drought stress. American Journal of Plant Physiology, 5: 338-349.

Ratzinger A., Riediger N., von Tiedemann A. and Karlovsky P. 2009. Salicylic acid and salicylic acid glucoside in xylem sap of Brassica napus infected with Verticillium longisporum. Journal of Plant Research, 122:571-579.

Reusche M., Truskina J., Thole K., Nagel L., Rindfleisch S., Tran Van T. and Braus-Stromeyer S. A. 2014. Infections with the vascular pathogens Verticillium longisporum and Verticillium dahliae induce distinct disease symptoms and differentially affect drought stress tolerance of Arabidopsis thaliana. Environmental and Experimental Botany, in press, Accepted manuscript.

Rygulla W., Friedt W., Seyis F., Lühs W., Eynck C., von Tiedemann A. and R. J. Snowdon. 2007a. Combination of resistance to Verticillium longisporum from zero erucic acid Brassica oleracea and oilseed Brassica rapa genotypes in resynthesized rapeseed (Brassica napus) lines. Plant Breeding, 126:596-602.

Rygulla W., Snowdon R. J., Eynck C., Koopmann B., von Tiedemann A., Lühs W. and Friedt W. 2007b. Broadening the genetic basis of Verticillium longisporum resistance in Brassica napus by interspecific hybridization. Phytopathology, 97:1391-1396.

Saadia M., Jamil A., Akram N. A. and Ashraf M.. 2012. A Study of proline metabolism in canola (Brassica napus L.) seedlings under salt stress. Molecules, 17: 5803-5815.

Sangtarash M. H., Qaderi M. M., Chinnappa C. C. and Reid D. M. 2009. Differential sensitivity of canola (Brassica napus) seedlings to ultraviolet-B radiation, water stress and abscisic acid. Environmental and Experimental Botany, 66:212-219.

Siebold M. 2012. Effects of rising air and soil temperatures on the life cycle of important pathogens in oilseed rape (Brassica napus L.) in Lower Saxony. PhD thesis. Georg-August-Universität Göttingen, Göttingen, Germany.

Subramanian S., Cho U. H., Keyes C. and Yu O. 2009. Distinct changes in soybean xylem sap proteome in response to pathogenic and symbiotic microbe interactions. BMC Plant Biology, 9(119):1-11.

Ullah, F., Bano A. and Nosheen A.. 2012. Effects of plant growth regulators on growth and oil quality of canola (Brassica napus L.) under drought stress. Pakistan Journal of Botany, 44(6): 1873-1880.

Veronese P., Narasimhan M. L., Stevenson R. A., Zhu J. K., Weller S. C., Subbarao K. V. and Bressan R. A. 2003. Identification of a locus controlling Verticillium disease symptom response in Arabidopsis thaliana. The Plant Journal, 35(5):574-87.

Wang E. L. H. and Bergeson G. B. 1974. Biochemical changes in root exudate and xylem sap of tomato plants infected with Meloidogyne incognita. Journal of Nematology, 6(4): 194-202. 
Xu P., Chen F., Mannas J. P., Feldman T., Sumner L. W. and Roossinck M. J. 2008. Virus infection improves drought tolerance. New Phytologist, 180: 911-921.

Xue X., Liu A. and Hua X. 2009. Proline accumulation and transcriptional regulation of proline biothesynthesis and degradation in Brassica napus. Biochemistry and Molecular Biology Reports, 42(1):28-34.

Yadeta K. A. and Thomma B. P. H. J. 2013. The xylem as battleground for plant hosts and vascular wilt pathogens. Frontiers in Plant Science, 4(97):1-12.

Yu S., Cao L., Zhou C. M., Zhang T. Q., Lian H., Sun Y., Wu J., Huang J., Wang G. and Wang J. W. 2013. Sugar is an endogenous cue for juvenile-to-adult phase transition in plants. elife, 2:e00269. 


\section{Summary}

Oilseed rape (Brassica napus) is one of the most important sources of vegetable oil in the world. Due to the growing demands in the biodiesel industry in particular, the global OSR production is gradually increasing since the last decade. Among other factors, the intensive production of OSR may have largely contributed to the emergence and increased economic importance of pest and diseases. One of the most important pathogens, Verticillium longisporum (VL), is a recently evolved vascular pathogen of crucifers. In recent periods, it has become a potential threat to OSR production in major OSR growing countries of the temperate region. This soil borne host-specific fungus causes foliar chlorosis, reduced growth, and premature senescence and ripening which ultimately leads to substantial yield losses. Unavailability of VL-effective fungicides and production of abundant and highly durable microsclerotia contributing to the soil inoculum are among the major factors that greatly hampers the management of VL. The only possible alternative management option available at present is the use of genotypes with enhanced resistance. The present study therefore focused on the identification of $B$. napus lines resistant to VL. Besides, the applicability of qPCR as an alternative method for the assessment of VL disease severity in the field was validated. Furthermore, mechanisms of cultivar related disease resistance and the significance of plant resistance mechanisms to VL under drought stress conditions were investigated.

Chapter two describes the resistance screening experiments conducted under greenhouse, outdoor and field conditions. Initially, a large number (>230) of B. napus DH lines and accessions were screened for VL resistance in multiple greenhouse experiments. Results of disease severity (AUDPC) and stunting effect assessments not only demonstrated the availability of VL-resistance in $B$. napus but also showed the presence of a wide range of variation in the level of resistance. Accordingly, B. napus lines that showed high degree of resistance in the greenhouse conditions were identified and recommended for further evaluation in the field. It was also found that the greenhouse resistance screening method used in this study, that involved the use of non-vernalized few-weeks old plants, provided consistent and reliable information. The method further enabled the screening of quite a large number of plants within a relatively small space and short period of time.

The outdoor screening experiment was conducted with the specific objective of identifying the sources/causes of variations in VL symptoms (mainly stunting and excessive branching) between greenhouse and field conditions. Results showed that stunting is significantly correlated with disease severity (genotype's susceptibility). This is in agreement with greenhouse observations and suggests the possible effect of type/source of inoculum and method of inoculation on the development of this 
symptom. In contrast, increased branching as a result of VL infection was genotype dependent and is apparently not related to susceptibility to VL.

In the field experiments, more than 80 best performing lines selected based on previous greenhouse screening results were evaluated in three different locations that vary in climatic factors, soil conditions and level of natural disease infestation. From the field trials conducted for three consecutive seasons (from 2010/11 to 2012/13), it was understood that plant genotype, seasonal variations and locational differences have a significant influence on the extent of disease development and the resulting response of plants to VL infection. Like in greenhouse conditions, significant differences among genotypes were observed in the field. Accordingly, best performing B. napus DH lines were identified and recommended for use as parent materials in future breeding programs that aim at integrating VL resistance in commercial OSR varieties. The other interesting observation from the field experiments was the substantial variation of disease severity across locations. The highest disease severity was observed in Svaöv followed by Fehmarn. In contrast, the least disease level was recorded in Göttingen, where unlike the other locations trials were conducted with additional artificial application of inoculum. Regarding seasonal variations, comparisons were not conclusive as no complete data sets were available. Nevertheless, compared to the later seasons, disease severity in $2010 / 10$ was relatively higher. More importantly, correlation analysis showed that disease index assessment results are poorly and only occasionally correlated across locations, years as well as with results of greenhouse experiments. In contrast, qPCR was the only field disease evaluation parameter that showed very strong correlations across locations. Furthermore, unlike disease index, this parameter was more significantly correlated to field disease index recordings in different locations and years, and with AUDPC and stunting effect results of greenhouse and outdoor experiments. With this method, detection of OSR infection in the field was possible as early as $\mathrm{BBCH} 65$ (50\% flowering stage). However, distinct separation of resistance and susceptible varieties and differentiation of infestation levels across locations was achieved at $\mathrm{BBCH} 80$. Hence, for reliable quantification of VL disease severity and identification of differential plant resistance by $\mathrm{qPCR}$ in the field, sampling between growth stages $\mathrm{BBCH} 70$ and $\mathrm{BBCH} 70$ are recommended. In conclusion, the use of qPCR method for detection and quantification of VL infection in OSR field samples has several merits over the use of post-harvest stubble disease index screening. The consistency and rapidity of this method and possibly the cost effectiveness in comparing plant resistance or assessing disease epidemics in the field is much more reliable than post- harvest stubble disease index assessment. Nevertheless, further improvement of this method to achieve early (in late autumn or early spring) detection of infection or disease severity will provide a more applicable and timely information. 
Chapter three mainly focused on the investigation of cultivar related VL-resistance factors in winter OSR xylem sap, an environment where the pathogen spends most part of its life cycle. Three $B$. napus genotypes with known differential resistance towards VL were used. Greenhouse studies were conducted to obtain xylem sap and verify resistance responses of the genotypes. In vitro bioassays and biochemical analyses were performed to study the effects of OSR xylem sap constituents on the growth of VL. From in vivo phenotypic (AUDPC and stunting effect) and molecular (qPCR) disease severity evaluations, the resistance of genotype SEM and susceptibility of Falcon was confirmed. Further evidences for the consistent response of these genotypes to VL-infection were obtained from the assessment of stem thickness and biomass yield. In vitro fungal growth analysis showed uniform growth of VL on xylem sap of $28 \mathrm{DPI}$ old susceptible and resistant genotypes. Similarly, comparisons of fungal growth in xylem sap of mock and VL-inoculated plants revealed the absence of infection induced VLresistance, which would contribute to significant reduction in VL growth. Quantification of total soluble protein content also showed no significant difference between xylem sap of resistant and susceptible genotypes. Even the infection-induced slight increase in protein concentration was similar in both susceptible and resistant genotypes. Interestingly, a time course independent study using the resistant genotype AVISO and susceptible cultivar Falcon indicated that regardless of plant resistance to $\mathrm{VL}$, xylem sap collected from older plants provide enhanced fungal growth. This phenomenon correlates with natural lifecycle of the pathogen on field grown OSR plants. The increased accumulation of xylem sap sugars in older plants could also be one of the possible explanations for enhanced growth of VL in xylem sap of older plants. This is a first study on functional analysis of cultivar related VL-resistance factors in winter OSR. It provided concrete evidence that OSR xylem sap, regardless of disease resistance, provides a favourable nutritional and chemical environment for the growth of VL. The slightly increased total soluble protein and sugar content in xylem sap of infected plants also demonstrates possible VL-induced changes in the composition or level of OSR xylem sap constituents. However, since these quantitative changes were not significantly different between resistant and susceptible genotypes, it can be concluded that soluble xylem sap constituents do not play a role as major resistance factors for cultivarrelated resistance of winter OSR against VL. This is in strong agreement with previous studies on the mechanisms of VL-resistance in OSR that demonstrated the significant role of cell wall bound metabolites and physical barriers. Further studies with large number of genotypes and assessment of other parameters such as effects of xylem sap constituents on fungal sporulation are suggested.

In the last chapter, the study on the response of $B$. napus to the combined effects of VL infection and drought stress was conducted. Previously, substantial amounts of vascular occlusions that obstruct 
xylem vessels have been detected in resistant VL-infected OSR genotypes. This mechanism of resistance to the pathogen may however alter the rate of water and nutrient transport and consequently plant response to drought stress. To investigate whether genotypic VL resistance is associated with a reduced drought tolerance, drought resistance of VL-resistant and susceptible winter OSR genotypes were studied in combination with infection with VL. Furthermore, the influence of drought stress on the response of plants to VL infection was investigated. This study was conducted in a controlled pot experiment where seedlings of the VL susceptible cultivar Falcon and the tolerant line SEM were inoculated with VL and exposed to three watering levels (optimum, moderate deficiency and severe deficiency i.e. watering at 100, 60 and 30\% field capacity). Analysis of disease parameters (AUDPC, stunting and qPCR) showed a significantly lower rate and level of disease development in the resistant genotype across all watering regimes. Likewise, regardless of the water supply at different field capacity levels, high disease severity and stunting effects were observed in the susceptible cultivar. Furthermore, the amount of fungal DNA was up to 31 fold in Falcon as compared to SEM. qPCR results showed that levels of fungal DNA were positively correlated with the intensity of drought stress. At 49 DPI, the respective average fungal DNA in dry hypocotyl tissue at 100,60 , and $30 \%$ field capacity was $27.1,29.0$ and $36.0 \mathrm{ng} / \mathrm{g}$ in SEM and 839.1, 1,032.4 and 1,096.4 ng/g in Falcon; indicating more pronounced effect of VL during drought stress, particularly on susceptible B. napus varieties. Significant changes in physiological parameters (gas exchange, relative water content, proline accumulation and water use efficiency) and up-regulation of drought stress marker genes confirmed the reaction of both genotypes to drought stress. On the other hand, neither VL alone nor its interaction with drought or the genotype had any significant effect on physiological parameters. Further comparisons of the drought induced physiological changes under mock- and VL-inoculation conditions showed a cultivar-independent trend of a slightly reduced impact of drought stress during VL infection. The main and interactive effects of VL and drought on biomass yield and other agronomic traits (stem diameter, branching and phenological growth stage) were significant but the magnitude of their impact was dependent on differential disease and physiological responses of the genotypes. In general, the consistent and interrelated results from ANOVA, correlation, regression and principal component analyses of the present comprehensive study not only proved that VL-resistance mechanisms have no additive negative consequence on plant performance under drought stress but also demonstrate effective functioning of the quantitative VLresistance mechanisms even under conditions of severe drought stress. Nevertheless, despite the stable VL-resistance under water deficit conditions and the slightly smaller effects of drought on infected plants, simultaneous exposure of OSR to both stresses can cause considerable yield loss. 
Appendices 
Appendix 2.1 List and descriptions of Brassica napus lines screened for Verticillium longisporum resistance under greenhouse conditions.

\begin{tabular}{|c|c|c|c|c|c|c|c|}
\hline No. & $\begin{array}{l}\text { Genotype } \\
\text { code }\end{array}$ & $\begin{array}{l}\text { Screening no. } \\
\text { /year }\end{array}$ & $\begin{array}{c}\text { Seed } \\
\text { company }\end{array}$ & No. & $\begin{array}{l}\text { Genotype } \\
\text { code }\end{array}$ & $\begin{array}{l}\text { Screening no. } \\
\text { /year }\end{array}$ & $\begin{array}{c}\text { Seed } \\
\text { company }\end{array}$ \\
\hline 1 & DSV $10-10$ & GHS42/2011 & DSV & 41 & SRG 204 & GHS42/2011 & SRG \\
\hline 2 & DSV 10-11 & GHS42/2011 & DSV & 42 & SRG 205 & GHS42/2011 & SRG \\
\hline 3 & DSV 10-12 & GHS42/2011 & DSV & 43 & SRG 206 & GHS42/2011 & SRG \\
\hline 4 & DSV 10-13 & GHS42/2011 & DSV & 44 & SRG 207 & GHS42/2011 & SRG \\
\hline 5 & DSV 10-14 & GHS42/2011 & DSV & 45 & SRG 208 & GHS42/2011 & SRG \\
\hline 6 & DSV 10-15 & GHS42/2011 & DSV & 46 & SRG 209 & GHS42/2011 & SRG \\
\hline 7 & DSV 10-16 & GHS42/2011 & DSV & 47 & SRG 210 & GHS42/2011 & SRG \\
\hline 8 & DSV $10-5$ & GHS42/2011 & DSV & 48 & SRG 211 & GHS42/2011 & SRG \\
\hline 9 & DSV $10-6$ & GHS42/2011 & DSV & 49 & SRG 212 & GHS42/2011 & SRG \\
\hline 10 & DSV 10-7 & GHS42/2011 & DSV & 50 & SRG 213 & GHS42/2011 & SRG \\
\hline 11 & DSV $10-8$ & GHS42/2011 & DSV & 51 & SW 309009 & GHS42/2011 & SW \\
\hline 12 & DSV 10-9 & GHS42/2011 & DSV & 52 & SW 309010 & GHS42/2011 & SW \\
\hline 13 & KWS 188 & GHS42/2011 & KWS & 53 & SW 309011 & GHS42/2011 & sw \\
\hline 14 & KWS 189 & GHS42/2011 & KWS & 54 & SW 309012 & GHS42/2011 & SW \\
\hline 15 & KWS 190 & GHS42/2011 & KWS & 55 & SW 309013 & GHS42/2011 & SW \\
\hline 16 & KWS 191 & GHS42/2011 & KWS & 56 & SW 309014 & GHS42/2011 & SW \\
\hline 17 & KWS 192 & GHS42/2011 & KWS & 57 & SW 309015 & GHS42/2011 & SW \\
\hline 18 & KWS 193 & GHS42/2011 & KWS & 58 & SW 309016 & GHS42/2011 & SW \\
\hline 19 & KWS 194 & GHS42/2011 & KWS & 59 & SW 309017 & GHS42/2011 & SW \\
\hline 20 & KWS 195 & GHS42/2011 & KWS & 60 & SW 309018 & GHS42/2011 & SW \\
\hline 21 & KWS 196 & GHS42/2011 & KWS & 61 & SW 309019 & GHS42/2011 & SW \\
\hline 22 & KWS 197 & GHS42/2011 & KWS & 62 & SW 309020 & GHS42/2011 & SW \\
\hline 23 & KWS 198 & GHS42/2011 & KWS & 63 & SYN VER 228 & GHS42/2011 & SYN \\
\hline 24 & KWS 199 & GHS42/2011 & KWS & 64 & SYN VER 229 & GHS42/2011 & SYN \\
\hline 25 & NICK 183 & GHS42/2011 & NICK & 65 & SYN VER 230 & GHS42/2011 & SYN \\
\hline 26 & NICK 184 & GHS42/2011 & NICK & 66 & SYN VER 231 & GHS42/2011 & SYN \\
\hline 27 & NPZ 198/08 & GHS42/2011 & NPZ & 67 & SYN VER 232 & GHS42/2011 & SYN \\
\hline 28 & NPZ 199/08 & GHS42/2011 & NPZ & 68 & SYN VER 233 & GHS42/2011 & SYN \\
\hline 29 & NPZ 200/08 & GHS42/2011 & NPZ & 69 & SYN VER 235 & GHS42/2011 & SYN \\
\hline 30 & NPZ 201/08 & GHS42/2011 & NPZ & 70 & SYN VER 236 & GHS42/2011 & SYN \\
\hline 31 & NPZ 202/08 & GHS42/2011 & NPZ & 71 & SYN VER 237 & GHS42/2011 & SYN \\
\hline 32 & NPZ 203/08 & GHS42/2011 & NPZ & 72 & SYN VER 239 & GHS42/2011 & SYN \\
\hline 33 & NPZ 204/08 & GHS42/2011 & NPZ & 73 & SYN VER 240 & GHS42/2011 & SYN \\
\hline 34 & NPZ 205/08 & GHS42/2011 & NPZ & 74 & WVB 203 & GHS42/2011 & WVB \\
\hline 35 & NPZ 206/08 & GHS42/2011 & NPZ & 75 & WVB 204 & GHS42/2011 & WVB \\
\hline 36 & NPZ 207/08 & GHS42/2011 & NPZ & 76 & WVB 205 & GHS42/2011 & WVB \\
\hline 37 & NPZ 208/08 & GHS42/2011 & NPZ & 77 & WVB 206 & GHS42/2011 & WVB \\
\hline 38 & NPZ 209/08 & GHS42/2011 & NPZ & 78 & WVB 207 & GHS42/2011 & WVB \\
\hline 39 & SRG 202 & GHS42/2011 & SRG & 79 & WVB 208 & GHS42/2011 & WVB \\
\hline 40 & SRG 203 & GHS42/2011 & SRG & 80 & WVB 209 & GHS42/2011 & WVB \\
\hline
\end{tabular}


Appendix 2.1 continued

\begin{tabular}{|c|c|c|c|c|c|c|c|}
\hline No. & $\begin{array}{l}\text { Genotype } \\
\text { code }\end{array}$ & $\begin{array}{l}\text { Screening no. } \\
\text { /year }\end{array}$ & $\begin{array}{c}\text { Seed } \\
\text { company }\end{array}$ & No. & $\begin{array}{l}\text { Genotype } \\
\text { code }\end{array}$ & $\begin{array}{c}\text { Screening no. } \\
\text { /year }\end{array}$ & $\begin{array}{c}\text { Seed } \\
\text { company }\end{array}$ \\
\hline 81 & WVB 210 & GHS42/2011 & WVB & 121 & SRG 221 & GHS43/2011 & SRG \\
\hline 82 & WVB 211 & GHS42/2011 & WVB & 122 & SRG 222 & GHS43/2011 & SRG \\
\hline 83 & WVB 212 & GHS42/2011 & WVB & 123 & SRG 223 & GHS43/2011 & SRG \\
\hline 84 & WVB 213 & GHS42/2011 & WVB & 124 & SW 08-309021 & GHS43/2011 & SW \\
\hline 85 & WVB 214 & GHS42/2011 & WVB & 125 & SW 08-309022 & GHS43/2011 & sw \\
\hline 86 & DSV 10-17 & GHS43/2011 & DSV & 126 & SW 08-309023 & GHS43/2011 & SW \\
\hline 87 & DSV $10-18$ & GHS43/2011 & DSV & 127 & SW 08-309024 & GHS43/2011 & sW \\
\hline 88 & DSV 10-19 & GHS43/2011 & DSV & 128 & SW 08-309025 & GHS43/2011 & SW \\
\hline 89 & DSV $10-20$ & GHS43/2011 & DSV & 129 & SW 08-309026 & GHS43/2011 & sW \\
\hline 90 & DSV 10-21 & GHS43/2011 & DSV & 130 & SW 08-309027 & GHS43/2011 & SW \\
\hline 91 & DSV 10-22 & GHS43/2011 & DSV & 131 & SW 08-309028 & GHS43/2011 & SW \\
\hline 92 & DSV 10-23 & GHS43/2011 & DSV & 132 & SW 08-309029 & GHS43/2011 & SW \\
\hline 93 & DSV $10-24$ & GHS43/2011 & DSV & 133 & SW 08-309030 & GHS43/2011 & SW \\
\hline 94 & DSV $10-25$ & GHS43/2011 & DSV & 134 & SYN VER 234 & GHS43/2011 & SYN \\
\hline 95 & DSV $10-26$ & GHS43/2011 & DSV & 135 & SYN VER 241 & GHS43/2011 & SYN \\
\hline 96 & KWS 200 & GHS43/2011 & KWS & 136 & SYN VER 242 & GHS43/2011 & SYN \\
\hline 97 & KWS 201 & GHS43/2011 & KWS & 137 & SYN VER 243 & GHS43/2011 & SYN \\
\hline 98 & KWS 202 & GHS43/2011 & KWS & 138 & SYN VER 244 & GHS43/2011 & SYN \\
\hline 99 & KWS 203 & GHS43/2011 & KWS & 139 & SYN VER 245 & GHS43/2011 & SYN \\
\hline 100 & KWS 204 & GHS43/2011 & KWS & 140 & SYN VER 246 & GHS43/2011 & SYN \\
\hline 101 & KWS 205 & GHS43/2011 & KWS & 141 & SYN VER 247 & GHS43/2011 & SYN \\
\hline 102 & KWS 206 & GHS43/2011 & KWS & 142 & SYN VER 248 & GHS43/2011 & SYN \\
\hline 103 & NPZ 210/08 & GHS43/2011 & NPZ & 143 & SYN VER 249 & GHS43/2011 & SYN \\
\hline 104 & NPZ 211/08 & GHS43/2011 & NPZ & 144 & SYN VER 250 & GHS43/2011 & SYN \\
\hline 105 & NPZ 212/08 & GHS43/2011 & NPZ & 145 & SYN VER 251 & GHS43/2011 & SYN \\
\hline 106 & NPZ 213/08 & GHS43/2011 & NPZ & 146 & WVB 215 & GHS43/2011 & WVB \\
\hline 107 & NPZ 214/08 & GHS43/2011 & NPZ & 147 & WVB 216 & GHS43/2011 & WVB \\
\hline 108 & NPZ 215/08 & GHS43/2011 & NPZ & 148 & WVB 217 & GHS43/2011 & WVB \\
\hline 109 & NPZ 216/08 & GHS43/2011 & NPZ & 149 & WVB 218 & GHS43/2011 & WVB \\
\hline 110 & NPZ 217/08 & GHS43/2011 & NPZ & 150 & WVB 219 & GHS43/2011 & WVB \\
\hline 111 & NPZ 218/08 & GHS43/2011 & NPZ & 151 & WVB 220 & GHS43/2011 & WVB \\
\hline 112 & NPZ 219/08 & GHS43/2011 & NPZ & 152 & WVB 221 & GHS43/2011 & WVB \\
\hline 113 & NPZ 220/08 & GHS43/2011 & NPZ & 153 & WVB 222 & GHS43/2011 & WVB \\
\hline 114 & SRG 214 & GHS43/2011 & SRG & 154 & WVB 223 & GHS43/2011 & WVB \\
\hline 115 & SRG 215 & GHS43/2011 & SRG & 155 & WVB 224 & GHS43/2011 & WVB \\
\hline 116 & SRG 216 & GHS43/2011 & SRG & 156 & DSV $10-27$ & GHS44/2012 & DSV \\
\hline 117 & SRG 217 & GHS43/2011 & SRG & 157 & DSV $10-28$ & GHS44/2012 & DSV \\
\hline 118 & SRG 218 & GHS43/2011 & SRG & 158 & DSV $10-29$ & GHS44/2012 & DSV \\
\hline 119 & SRG 219 & GHS43/2011 & SRG & 159 & DSV $10-30$ & GHS44/2012 & DSV \\
\hline 120 & SRG 220 & GHS43/2011 & SRG & 160 & DSV 10-31 & GHS44/2012 & DSV \\
\hline
\end{tabular}


Appendix 2.1 continued

\begin{tabular}{|c|c|c|c|c|c|c|c|}
\hline No. & $\begin{array}{l}\text { Genotype } \\
\text { code }\end{array}$ & $\begin{array}{c}\text { Screening no. } \\
\text { /year }\end{array}$ & $\begin{array}{c}\text { Seed } \\
\text { company }\end{array}$ & No. & $\begin{array}{l}\text { Genotype } \\
\text { code }\end{array}$ & $\begin{array}{c}\text { Screening no. } \\
\text { /year }\end{array}$ & $\begin{array}{c}\text { Seed } \\
\text { company }\end{array}$ \\
\hline 161 & DSV $10-32$ & GHS44/2012 & DSV & 200 & DSV $10-41$ & GHS46/2012 & DSV \\
\hline 162 & DSV 10-33 & GHS44/2012 & DSV & 201 & DSV $10-42$ & GHS46/2012 & DSV \\
\hline 163 & DSV 10-34 & GHS44/2012 & DSV & 202 & DSV $10-43$ & GHS46/2012 & DSV \\
\hline 164 & NICK 185 & GHS44/2012 & NICK & 203 & DSV $10-44$ & GHS46/2012 & DSV \\
\hline 165 & NICK 186 & GHS44/2012 & NICK & 204 & DSV $10-45$ & GHS46/2012 & DSV \\
\hline 166 & NICK 187 & GHS44/2012 & NICK & 205 & DSV $10-46$ & GHS46/2012 & DSV \\
\hline 167 & NICK 188 & GHS44/2012 & NICK & 206 & KWS 217 & GHS46/2012 & KWS \\
\hline 168 & NICK 189 & GHS44/2012 & NICK & 207 & KWS 218 & GHS46/2012 & KWS \\
\hline 169 & NICK 190 & GHS44/2012 & NICK & 208 & KWS 219 & GHS46/2012 & KWS \\
\hline 170 & NICK 191 & GHS44/2012 & NICK & 209 & KWS 220 & GHS46/2012 & KWS \\
\hline 171 & NICK 192 & GHS44/2012 & NICK & 210 & KWS 221 & GHS46/2012 & KWS \\
\hline 172 & SRG 224 & GHS44/2012 & SRG & 211 & KWS 222 & GHS46/2012 & KWS \\
\hline 173 & SRG 225 & GHS44/2012 & SRG & 212 & NICK 194 & GHS46/2012 & NICK \\
\hline 174 & SRG 226 & GHS44/2012 & SRG & 213 & NICK 195 & GHS46/2012 & NICK \\
\hline 175 & SRG 227 & GHS44/2012 & SRG & 214 & NICK 196 & GHS46/2012 & NICK \\
\hline 176 & SRG 228 & GHS44/2012 & SRG & 215 & NICK 197 & GHS46/2012 & NICK \\
\hline 177 & SRG 229 & GHS44/2012 & SRG & 216 & NICK 198 & GHS46/2012 & NICK \\
\hline 178 & SW 08-309026 & GHS44/2012 & sW & 217 & NICK 199 & GHS46/2012 & NICK \\
\hline 179 & SW 08-309031 & GHS44/2012 & sW & 218 & SW 12-1 & GHS46/2012 & sW \\
\hline 180 & SW 08-309032 & GHS44/2012 & sW & 219 & SW 12-2 & GHS46/2012 & sW \\
\hline 181 & SW 08-309033 & GHS44/2012 & sW & 220 & SW 12-3 & GHS46/2012 & SW \\
\hline 182 & SW 08-309034 & GHS44/2012 & sW & 221 & SW 12-4 & GHS46/2012 & sW \\
\hline 183 & SW 08-309035 & GHS44/2012 & sW & 222 & SW 12-5 & GHS46/2012 & sw \\
\hline 184 & SYN VER 252 & GHS44/2012 & SYN & 223 & SW 12-6 & GHS46/2012 & sw \\
\hline 185 & SYN VER 253 & GHS44/2012 & SYN & 224 & SYN VER 260 & GHS46/2012 & SYN \\
\hline 186 & SYN VER 254 & GHS44/2012 & SYN & 225 & SYN VER 261 & GHS46/2012 & SYN \\
\hline 187 & SYN VER 255 & GHS44/2012 & SYN & 226 & SYN VER 262 & GHS46/2012 & SYN \\
\hline 188 & SYN VER 256 & GHS44/2012 & SYN & 227 & SYN VER 263 & GHS46/2012 & SYN \\
\hline 189 & SYN VER 257 & GHS44/2012 & SYN & 228 & SYN VER 264 & GHS46/2012 & SYN \\
\hline 190 & SYN VER 258 & GHS44/2012 & SYN & 229 & SYN VER 265 & GHS46/2012 & SYN \\
\hline 191 & SYN VER 259 & GHS44/2012 & SYN & 230 & WVB 225 & GHS46/2012 & WVB \\
\hline 192 & WVB 225 & GHS44/2012 & WVB & 231 & WVB 226 & GHS46/2012 & WVB \\
\hline 193 & WVB 226 & GHS44/2012 & WVB & 232 & WVB 227 & GHS46/2012 & WVB \\
\hline 194 & WVB 227 & GHS44/2012 & WVB & 233 & WVB 228 & GHS46/2012 & WVB \\
\hline 195 & WVB 228 & GHS44/2012 & WVB & 234 & WVB 229 & GHS46/2012 & WVB \\
\hline 196 & WVB 229 & GHS44/2012 & WVB & 235 & WVB 230 & GHS46/2012 & WVB \\
\hline 197 & WVB 230 & GHS44/2012 & WVB & 236 & Express $^{(\mathrm{RC})}$ & All trials & NPZ \\
\hline 198 & WVB 231 & GHS44/2012 & WVB & 237 & Falcon ${ }^{(\mathrm{SC})}$ & All trials & NPZ \\
\hline 199 & WVB 232 & GHS44/2012 & WVB & & & & \\
\hline
\end{tabular}

DSV, Deutsche Saatveredelung AG. KWS, KWS SAAT AG. NICK, Limagrain GmbH. NPZ, NorddeutschePflanzenzucht Hans-Georg Lembke KG. SRG, Raps GbR Saatzucht Lundsgaard. SW, Lantmännen SW Seed. SYN, Syngenta Seeds $\mathrm{GmbH}$. WVB, W. von Borries-Eckendorf GmbH \& Co. VL, Verticillium longisporum. (RC), resistant reference variety. (SC), susceptible reference variety. 
Appendix 2.2 List and descriptions of Brassica napus lines evaluated for Verticillium longisporum resistance under field conditions.

\begin{tabular}{|c|c|c|c|c|c|c|c|}
\hline No. & $\begin{array}{l}\text { Genotype } \\
\text { code }\end{array}$ & Season & $\begin{array}{c}\text { Seed } \\
\text { company }\end{array}$ & No. & $\begin{array}{l}\text { Genotype } \\
\text { code }\end{array}$ & Season & $\begin{array}{c}\text { Seed } \\
\text { company }\end{array}$ \\
\hline 1 & SEM 05-500256 & All & SW & 43 & DSV-DH-Ver-7(1575-2) & $2011 / 12$ & DSV \\
\hline 2 & SW 08-190001-1 ${ }^{*}$ & All & SW & 44 & DSV-DH-Ver-8(1605-2) ${ }^{*}$ & $2011 / 12$ & DSV \\
\hline 3 & SW 08-190001-6 ${ }^{*}$ & All & SW & 45 & DSV-DH-Ver-9(1605-2) & $2011 / 12$ & DSV \\
\hline 4 & SW 08-190001-7 & All & SW & 46 & DSV-DH-Ver-10(1605-2) & $2011 / 12$ & DSV \\
\hline 5 & SW 08-190001-11 ${ }^{*}$ & All & SW & 47 & DSV-DH-Ver-11(1605-2) & $2011 / 12$ & DSV \\
\hline 6 & SW 08-190001-12* & All & SW & 48 & DSV-DH-Ver-12(1605-2) & $2011 / 12$ & DSV \\
\hline 7 & SW 08-190001-25* & All & SW & 49 & DSV-DH-Ver-13(1605-1) & $2011 / 12$ & DSV \\
\hline 8 & SW 08-190002-1 ${ }^{*}$ & All & SW & 50 & DSV-DH-Ver-14(1605-2) & $2011 / 12$ & DSV \\
\hline 9 & SW 08-190002-5 & All & SW & 51 & DSV-DH-Ver-15(1605-2) & $2011 / 12$ & DSV \\
\hline 10 & SW $08-190002-8^{*}$ & All & SW & 52 & DSV-DH-Ver-16(1605-2) & $2011 / 12$ & DSV \\
\hline 11 & SW 08-190002-9* & All & sW & 53 & DSV-DH-Ver-17(1605-2) & $2011 / 12$ & DSV \\
\hline 12 & SW 08-190002-11 ${ }^{*}$ & All & sW & 54 & DSV-DH-Ver-18(1605-2) & $2011 / 12$ & DSV \\
\hline 13 & SW 08-190002-16* & All & SW & 55 & DSV-DH-Ver-19(1605-2) & $2011 / 12$ & DSV \\
\hline 14 & SW 08-190002-18* & All & SW & 56 & DSV-DH-Ver-20(1605-3) ${ }^{*}$ & $2011 / 12$ & DSV \\
\hline 15 & $\mathrm{DH} 7^{*}$ & $2010 / 11$ & $\mathrm{JLU}$ & 57 & DSV-DH-Ver-21(1605-1) & $2011 / 12$ & DSV \\
\hline 16 & $\mathrm{DH} 16^{*}$ & $2010 / 11$ & $\mathrm{JLU}$ & 58 & DSV-DH-Ver-22(1605-2) & $2011 / 12$ & DSV \\
\hline 17 & $\mathrm{DH} 24^{*}$ & $2010 / 11$ & JLU & 59 & DM 12-01 & $2012 / 13$ & DM \\
\hline 18 & DH $69^{*}$ & $2010 / 11$ & $\mathrm{JLU}$ & 60 & DM 12-02 & $2012 / 13$ & $\mathrm{DM}$ \\
\hline 19 & $\mathrm{DH} 88^{*}$ & $2010 / 11$ & $\mathrm{JLU}$ & 61 & DSV 1 & $2012 / 13$ & DSV \\
\hline 20 & $\mathrm{DH} 101^{*}$ & $2010 / 11$ & JLU & 62 & DSV 2 & $2012 / 13$ & DSV \\
\hline 21 & $\mathrm{DH} 108^{*}$ & $2010 / 11$ & JLU & 63 & DSV 3 & $2012 / 13$ & DSV \\
\hline 22 & $\mathrm{DH} 118^{*}$ & $2010 / 11$ & JLU & 64 & KWS 187 & $2012 / 13$ & KWS \\
\hline 23 & $\mathrm{DH} 123^{*}$ & $2010 / 11$ & $\mathrm{JLU}$ & 65 & KWS 195 & $2012 / 13$ & KWS \\
\hline 24 & $\mathrm{DH} 126^{*}$ & $2010 / 11$ & JLU & 66 & KWS 200 & $2012 / 13$ & KWS \\
\hline 25 & $\mathrm{DH} 138^{*}$ & $2010 / 11$ & $\mathrm{JLU}$ & 67 & NICK 219 & $2012 / 13$ & NICK \\
\hline 26 & $\mathrm{DH} 140^{*}$ & $2010 / 11$ & $\mathrm{JLU}$ & 68 & NICK 220 & $2012 / 13$ & NICK \\
\hline 27 & DH $141^{*}$ & $2010 / 11$ & $\mathrm{JLU}$ & 69 & NICK 221 & $2012 / 13$ & NICK \\
\hline 28 & $\mathrm{DH} 152^{*}$ & $2010 / 11$ & $\mathrm{JLU}$ & 70 & SW 08-190001-10* & $2012 / 13$ & SW \\
\hline 29 & $\mathrm{DH} 165^{*}$ & $2010 / 11$ & $\mathrm{JLU}$ & 71 & SW 08-190002-2* & $2012 / 13$ & sW \\
\hline 30 & DH $179^{*}$ & $2010 / 11$ & $\mathrm{JLU}$ & 72 & SW $08-190002-17^{*}$ & $2012 / 13$ & SW \\
\hline 31 & $\mathrm{DH} 196^{*}$ & $2010 / 11$ & JLU & 73 & SW 08-190002-25 & $2012 / 13$ & SW \\
\hline 32 & $\mathrm{DH} 272^{*}$ & $2010 / 11$ & JLU & 74 & SW 12-1 & $2012 / 13$ & SW \\
\hline 33 & $\mathrm{DH} 282^{*}$ & $2010 / 11$ & $\mathrm{JLU}$ & 75 & SW $12-2$ & $2012 / 13$ & SW \\
\hline 34 & $\mathrm{DH} 290^{*}$ & $2010 / 11$ & JLU & 76 & SW 12-3 & $2012 / 13$ & sW \\
\hline 35 & $\mathrm{DH} 294^{*}$ & $2010 / 11$ & JLU & 77 & SW $12-4$ & $2012 / 13$ & sW \\
\hline 36 & $\mathrm{DH} 359^{*}$ & $2010 / 11$ & $\mathrm{JLU}$ & 78 & SYN 09033483 & $2012 / 13$ & SYN \\
\hline 37 & DSV-DH-Ver-1(1575-1) & $2011 / 12$ & DSV & 79 & SYN 10091232 & $2012 / 13$ & SYN \\
\hline 38 & DSV-DH-Ver-2(1575-2) & $2011 / 12$ & DSV & 80 & SYN 11091465 & $2012 / 13$ & SYN \\
\hline 39 & DSV-DH-Ver-3(1575-2) & $2011 / 12$ & DSV & 81 & Express $^{(\mathrm{RC})}$ & All & NPZ \\
\hline 40 & DSV-DH-Ver-4(1575-3) & $2011 / 12$ & DSV & 82 & Falcon $^{(\mathrm{SC})}$ & All & NPZ \\
\hline 41 & DSV-DH-Ver-5(1575-1) & $2011 / 12$ & DSV & 83 & Laser $^{(\mathrm{SC})}$ & All & SYN \\
\hline 42 & DSV-DH-Ver-6(1575-1) ${ }^{*}$ & $2011 / 12$ & DSV & 84 & Oase $e^{(R C)}$ & All & DSV \\
\hline
\end{tabular}

Key: DM, Dieckmann GmbH \& Co. KG. DSV, Deutsche Saatveredelung AG. JLU, Justus-Liebig-University of Gießen, department of Plant breeding. KWS, KWS SAAT AG. NICK, Limagrain GmbH. NPZ, NorddeutschePflanzenzucht Hans-Georg Lembke KG. SW, Lantmännen SW Seed. SYN, Syngenta Seeds GmbH. VL, Verticillium longisporum. (RC), resistant control. (S), susceptible control. *, Double haploid line. 
Appendix 2.3 Response of Brassica napus lines to Verticillium longisporum under greenhouse conditions as measured by AUDPC and relative stunting.

\begin{tabular}{|c|c|c|c|c|}
\hline No. & Genotype code & $\begin{array}{l}\text { Normalized net } \\
\text { AUDPC (28 DPI) }\end{array}$ & $\begin{array}{l}\text { Normalized } \\
\text { relative stunting (28DPI) }\end{array}$ & $\begin{array}{l}\text { Experiment } \\
\text { No. }\end{array}$ \\
\hline 1 & NICK 191* & $-0.20 \pm 0.53$ & $0.03 \pm 0.74$ & GHS44 \\
\hline 2 & NICK $189 *$ & $-0.09 \pm 0.52$ & $0.23 \pm 0.65$ & GHS44 \\
\hline 3 & NICK 188 & $-0.04 \pm 0.24$ & $0.34 \pm 0.40$ & GHS44 \\
\hline 4 & DSV 10-33* & $0.02 \pm 0.16$ & $0.12 \pm 0.41$ & GHS44 \\
\hline 5 & NICK $186^{*}$ & $0.02 \pm 0.26$ & $0.11 \pm 0.63$ & GHS44 \\
\hline 6 & DSV $10-30 *$ & $0.04 \pm 0.68$ & $0.32 \pm 0.55$ & GHS44 \\
\hline 7 & NICK 190* & $0.04 \pm 0.42$ & $0.10 \pm 0.30$ & GHS44 \\
\hline 8 & DSV 10-32* & $0.07 \pm 0.72$ & $0.26 \pm 0.50$ & GHS44 \\
\hline 9 & SW 08-309026* & $0.07 \pm 0.51$ & $-0.13 \pm 0.67$ & GHS44 \\
\hline 10 & DSV 10-27 & $0.16 \pm 0.61$ & $0.43 \pm 0.59$ & GHS44 \\
\hline 11 & SRG 227 & $0.16 \pm 0.85$ & $0.71 \pm 0.44$ & GHS44 \\
\hline 12 & SW 08-309029* & $0.18 \pm 0.23$ & $-0.18+0.16$ & GHS43 \\
\hline 13 & NICK $187^{*}$ & $0.18+0.32$ & $0.19 \pm 0.36$ & GHS44 \\
\hline 14 & SYN VER 250 & $0.18 \pm 0.22$ & $0.58 \pm 0.20$ & GHS43 \\
\hline 15 & SRG 225 & $0.20 \pm 0.39$ & $0.63 \pm 0.43$ & GHS44 \\
\hline 16 & SRG 228* & $0.20 \pm 0.62$ & $0.09 \pm 0.55$ & GHS44 \\
\hline 17 & WVB 232 & $0.20 \pm 2.07$ & $1.31 \pm 1.78$ & GHS44 \\
\hline 18 & NICK $184^{*}$ & $0.24 \pm 0.11$ & $0.14 \pm 0.25$ & GHS42 \\
\hline 19 & WVB 231* & $0.25 \pm 0.35$ & $0.22 \pm 0.55$ & GHS44 \\
\hline 20 & SW 08-309033* & $0.27 \pm 0.28$ & $0.15+0.38$ & GHS44 \\
\hline 21 & SW 08-309035 & $0.29 \pm 0.35$ & $1.02+0.52$ & GHS44 \\
\hline 22 & NICK 192* & $0.29 \pm 0.42$ & $0.30 \pm 0.44$ & GHS44 \\
\hline 23 & SW 309016* & $0.29 \pm 0.07$ & $0.30 \pm 0.09$ & GHS42 \\
\hline 24 & SW 08-309024* & $0.31 \pm 0.18$ & $-0.08 \pm 0.16$ & GHS43 \\
\hline 25 & SW 08-309030* & $0.31 \pm 0.29$ & $0.03 \pm 0.14$ & GHS43 \\
\hline 26 & DSV 10-29* & $0.31 \pm 0.43$ & $-0.44 \pm 0.61$ & GHS44 \\
\hline 27 & DSV 10-28* & $0.31 \pm 0.54$ & $0.22 \pm 0.54$ & GHS44 \\
\hline 28 & WVB 225 & $0.34 \pm 0.63$ & $1.23 \pm 0.72$ & GHS44 \\
\hline 29 & WVB 226 & $0.34 \pm 1.04$ & $1.03 \pm 0.77$ & GHS44 \\
\hline 30 & SW 309019* & $0.34 \pm 0.07$ & $0.09 \pm 0.13$ & GHS42 \\
\hline 31 & NPZ 219/08* & $0.35 \pm 0.17$ & $0.33 \pm 0.21$ & GHS43 \\
\hline 32 & SYN VER 248 & $0.35 \pm 0.18$ & $0.57 \pm 0.10$ & GHS43 \\
\hline 33 & KWS 195 & $0.36 \pm 0.12$ & $0.56 \pm 0.22$ & GHS42 \\
\hline 34 & KWS 200* & $0.36 \pm 0.14$ & $0.20 \pm 0.17$ & GHS43 \\
\hline 35 & WVB 228 & $0.36 \pm 0.40$ & $0.45 \pm 0.52$ & GHS44 \\
\hline 36 & WVB 230 & $0.36 \pm 0.65$ & $0.79 \pm 0.48$ & GHS44 \\
\hline 37 & NPZ 199/08 & $0.36 \pm 0.20$ & $0.58 \pm 0.30$ & GHS42 \\
\hline 38 & SW 12-3 & $0.37 \pm 0.26$ & $0.54 \pm 0.33$ & GHS46 \\
\hline 39 & WVB 227 & $0.38 \pm 0.47$ & $1.29 \pm 0.94$ & GHS44 \\
\hline 40 & SW 309018 & $0.40+0.08$ & $0.25+0.07$ & GHS42 \\
\hline
\end{tabular}


Appendix 2.3 continued

\begin{tabular}{|c|c|c|c|c|}
\hline No. & Genotype code & $\begin{array}{l}\text { Normalized net } \\
\text { AUDPC }\end{array}$ & $\begin{array}{c}\text { Normalized } \\
\text { relative stunting }\end{array}$ & $\begin{array}{c}\text { Experiment } \\
\text { No. }\end{array}$ \\
\hline 41 & NPZ 217/08 & $0.40 \pm 0.13$ & $0.06 \pm 0.14$ & GHS43 \\
\hline 42 & DSV $10-26$ & $0.40 \pm 0.17$ & $0.52 \pm 0.30$ & GHS43 \\
\hline 43 & DSV $10-23$ & $0.41 \pm 0.14$ & $0.21 \pm 0.19$ & GHS43 \\
\hline 44 & DSV 10-9 & $0.41 \pm 0.19$ & $0.50 \pm 0.24$ & GHS42 \\
\hline 45 & WVB 207 & $0.41+0.15$ & $0.51 \pm 0.27$ & GHS42 \\
\hline 46 & NICK 195 & $0.41 \pm 0.19$ & $0.43 \pm 0.29$ & GHS46 \\
\hline 47 & NPZ 216/08 & $0.42 \pm 0.18$ & $0.43 \pm 0.16$ & GHS43 \\
\hline 48 & SYN VER 264 & $0.42 \pm 0.24$ & $0.63 \pm 0.20$ & GHS46 \\
\hline 49 & NPZ 203/08 & $0.42 \pm 0.26$ & $0.47 \pm 0.13$ & GHS42 \\
\hline 50 & SRG 211 & $0.43 \pm 0.13$ & $0.64 \pm 0.09$ & GHS42 \\
\hline 51 & SRG 221 & $0.44 \pm 0.15$ & $0.32 \pm 0.17$ & GHS43 \\
\hline 52 & SW 309010 & $0.45 \pm 0.07$ & $0.44 \pm 0.12$ & GHS42 \\
\hline 53 & SW 309017 & $0.45 \pm 0.06$ & $0.38 \pm 0.08$ & GHS42 \\
\hline 54 & SW 12-6 & $0.45 \pm 0.36$ & $0.49 \pm 0.25$ & GHS46 \\
\hline 55 & KWS 188 & $0.45 \pm 0.15$ & $0.48 \pm 0.21$ & GHS42 \\
\hline 56 & SW 309012 & $0.46 \pm 0.08$ & $0.65 \pm 0.10$ & GHS42 \\
\hline 57 & SW 08-309028 & $0.46 \pm 0.26$ & $0.03 \pm 0.15$ & GHS43 \\
\hline 58 & SW 08-309027 & $0.46 \pm 0.16$ & $0.15 \pm 0.14$ & GHS43 \\
\hline 59 & SYN VER 251 & $0.46 \pm 0.28$ & $0.81 \pm 0.13$ & GHS43 \\
\hline 60 & WVB 210 & $0.46 \pm 0.10$ & $0.51 \pm 0.19$ & GHS42 \\
\hline 61 & SW 12-4 & $0.47 \pm 0.23$ & $0.35 \pm 0.27$ & GHS46 \\
\hline 62 & DSV 10-34 & $0.47 \pm 0.41$ & $0.69 \pm 0.74$ & GHS44 \\
\hline 63 & KWS 201 & $0.47 \pm 0.27$ & $0.32 \pm 0.33$ & GHS43 \\
\hline 64 & SW 08-309022 & $0.48 \pm 0.32$ & $0.05 \pm 0.08$ & GHS43 \\
\hline 65 & NPZ 198/08 & $0.48 \pm 0.14$ & $0.53 \pm 0.10$ & GHS42 \\
\hline 66 & SW 309014 & $0.49 \pm 0.07$ & $0.38 \pm 0.14$ & GHS42 \\
\hline 67 & NPZ 211/08 & $0.49 \pm 0.20$ & $0.43 \pm 0.21$ & GHS43 \\
\hline 68 & SW 12-1 & $0.49 \pm 0.24$ & $0.53 \pm 0.43$ & GHS46 \\
\hline 69 & SW 309015 & $0.50 \pm 0.09$ & $0.32 \pm 0.16$ & GHS42 \\
\hline 70 & WVB 208 & $0.50 \pm 0.10$ & $0.62 \pm 0.19$ & GHS42 \\
\hline 71 & KWS 202 & $0.51 \pm 0.30$ & $0.37 \pm 0.21$ & GHS43 \\
\hline 72 & DSV 10-17 & $0.51 \pm 0.29$ & $0.64 \pm 0.33$ & GHS43 \\
\hline 73 & DSV 10-6 & $0.51 \pm 0.26$ & $0.60 \pm 0.31$ & GHS42 \\
\hline 74 & SYN VER 262 & $0.52 \pm 0.33$ & $0.62 \pm 0.36$ & GHS46 \\
\hline 75 & NICK 194 & $0.52 \pm 0.18$ & $0.62 \pm 0.29$ & GHS46 \\
\hline 76 & SYN VER 256 & $0.52 \pm 0.50$ & $1.68 \pm 0.96$ & GHS44 \\
\hline 77 & SW 08-309026* & $0.52 \pm 0.14$ & $0.11 \pm 0.09$ & GHS43 \\
\hline 78 & SW 309009 & $0.52 \pm 0.18$ & $0.68 \pm 0.14$ & GHS42 \\
\hline 79 & NICK 197 & $0.52 \pm 0.29$ & $0.81 \pm 0.24$ & GHS46 \\
\hline 80 & WVB 205 & $0.52 \pm 0.20$ & $0.64 \pm 0.27$ & GHS42 \\
\hline
\end{tabular}


Appendix 2.3 continued

\begin{tabular}{|c|c|c|c|c|}
\hline No. & Genotype code & $\begin{array}{l}\text { Normalized net } \\
\text { AUDPC }\end{array}$ & $\begin{array}{c}\text { Normalized } \\
\text { relative stunting }\end{array}$ & Experiment No. \\
\hline 81 & SYN VER 261 & $0.53 \pm 0.18$ & $0.86 \pm 0.27$ & GHS46 \\
\hline 82 & KWS 189 & $0.53 \pm 0.10$ & $0.79 \pm 0.09$ & GHS42 \\
\hline 83 & WVB 230 & $0.54 \pm 0.14$ & $0.36 \pm 0.20$ & GHS46 \\
\hline 84 & SW 309013 & $0.54 \pm 0.19$ & $0.93+0.28$ & GHS42 \\
\hline 85 & WVB 203 & $0.54 \pm 0.20$ & $0.68 \pm 0.30$ & GHS42 \\
\hline 86 & NPZ 204/08 & $0.54 \pm 0.33$ & $0.68 \pm 0.26$ & GHS42 \\
\hline 87 & DSV $10-12$ & $0.54 \pm 0.12$ & $0.79+0.17$ & GHS42 \\
\hline 88 & SRG 213 & $0.56 \pm 0.19$ & $0.92 \pm 0.25$ & GHS42 \\
\hline 89 & SRG 226 & $0.56 \pm 0.92$ & $0.17 \pm 0.39$ & GHS44 \\
\hline 90 & SYN VER 231 & $0.57 \pm 0.23$ & $0.89 \pm 0.30$ & GHS42 \\
\hline 91 & DSV $10-11$ & $0.58 \pm 0.21$ & $0.42 \pm 0.17$ & GHS42 \\
\hline 92 & Express & $0.58 \pm 0.31$ & $0.53 \pm 0.21$ & Resistant standard \\
\hline 93 & SRG 204 & $0.59 \pm 0.10$ & $0.57 \pm 0.17$ & GHS42 \\
\hline 94 & SW 08-309025 & $0.59 \pm 0.21$ & $0.33 \pm 0.14$ & GHS43 \\
\hline 95 & KWS 198 & $0.60 \pm 0.25$ & $0.72 \pm 0.37$ & GHS42 \\
\hline 96 & SRG 219 & $0.61 \pm 0.20$ & $0.38 \pm 0.14$ & GHS43 \\
\hline 97 & SYN VER 260 & $0.62+0.19$ & $0.74+0.19$ & GHS46 \\
\hline 98 & SW 309020 & $0.62 \pm 0.17$ & $0.65 \pm 0.21$ & GHS42 \\
\hline 99 & SYN VER 237 & $0.62 \pm 0.22$ & $0.98 \pm 0.22$ & GHS42 \\
\hline 100 & SRG 222 & $0.62 \pm 0.23$ & $0.34 \pm 0.20$ & GHS43 \\
\hline 101 & SRG 217 & $0.62 \pm 0.29$ & $0.69 \pm 0.40$ & GHS43 \\
\hline 102 & SRG 209 & $0.62 \pm 0.22$ & $0.59 \pm 0.15$ & GHS42 \\
\hline 103 & DSV $10-13$ & $0.63 \pm 0.22$ & $1.03 \pm 0.28$ & GHS42 \\
\hline 104 & WVB 227 & $0.63 \pm 0.15$ & $0.36 \pm 0.12$ & GHS46 \\
\hline 105 & SRG 210 & $0.63 \pm 0.18$ & $0.94 \pm 0.17$ & GHS42 \\
\hline 106 & SYN VER 233 & $0.63 \pm 0.15$ & $0.92 \pm 0.28$ & GHS42 \\
\hline 107 & SW 08-309021 & $0.64 \pm 0.22$ & $-0.16+0.11$ & GHS43 \\
\hline 108 & DSV $10-46$ & $0.64 \pm 0.09$ & $0.81 \pm 0.17$ & GHS46 \\
\hline 109 & WVB 225 & $0.64 \pm 0.18$ & $0.61 \pm 0.29$ & GHS46 \\
\hline 110 & NPZ 220/08 & $0.64 \pm 0.19$ & $0.99 \pm 0.18$ & GHS43 \\
\hline 111 & SRG 205 & $0.64 \pm 0.13$ & $0.88 \pm 0.18$ & GHS42 \\
\hline 112 & WVB 209 & $0.64 \pm 0.19$ & $0.84 \pm 0.25$ & GHS42 \\
\hline 113 & SW 309011 & $0.65 \pm 0.19$ & $0.69 \pm 0.28$ & GHS42 \\
\hline 114 & SW 08-309034 & $0.65 \pm 0.56$ & $0.73 \pm 0.57$ & GHS44 \\
\hline 115 & WVB 211 & $0.65 \pm 0.12$ & $0.59 \pm 0.20$ & GHS42 \\
\hline 116 & NPZ 212/08 & $0.66 \pm 0.25$ & $0.47 \pm 0.24$ & GHS43 \\
\hline 117 & SYN VER 246 & $0.66 \pm 0.36$ & $0.92 \pm 0.28$ & GHS43 \\
\hline 118 & KWS 194 & $0.66 \pm 0.15$ & $0.77 \pm 0.23$ & GHS42 \\
\hline 119 & DSV $10-7$ & $0.66 \pm 0.18$ & $0.96 \pm 0.30$ & GHS42 \\
\hline 120 & SRG 212 & $0.67 \pm 0.18$ & $1.16+0.14$ & GHS42 \\
\hline
\end{tabular}


Appendix 2.3 continued

\begin{tabular}{|c|c|c|c|c|}
\hline No. & Genotype code & $\begin{array}{l}\text { Normalized net } \\
\text { AUDPC }\end{array}$ & $\begin{array}{c}\text { Normalized } \\
\text { relative stunting }\end{array}$ & $\begin{array}{l}\text { Experiment } \\
\text { No. }\end{array}$ \\
\hline 121 & KWS 197 & $0.67 \pm 0.24$ & $0.94 \pm 0.22$ & GHS42 \\
\hline 122 & SW 08-309023 & $0.67 \pm 0.25$ & $0.09 \pm 0.16$ & GHS43 \\
\hline 123 & DSV $10-10$ & $0.67 \pm 0.36$ & $0.90 \pm 0.35$ & GHS42 \\
\hline 124 & SYN VER 232 & $0.67 \pm 0.29$ & $0.91 \pm 0.33$ & GHS42 \\
\hline 125 & NPZ 200/08 & $0.68 \pm 0.22$ & $0.52 \pm 0.21$ & GHS42 \\
\hline 126 & WVB 214 & $0.69 \pm 0.29$ & $0.68 \pm 0.26$ & GHS42 \\
\hline 127 & NPZ 215/08 & $0.70 \pm 0.22$ & $0.31 \pm 0.16$ & GHS43 \\
\hline 128 & SRG 216 & $0.70 \pm 0.09$ & $0.47 \pm 0.06$ & GHS43 \\
\hline 129 & WVB 228 & $0.70 \pm 0.11$ & $0.21 \pm 0.28$ & GHS46 \\
\hline 130 & SRG 202 & $0.71 \pm 0.21$ & $0.70 \pm 0.18$ & GHS42 \\
\hline 131 & WVB 204 & $0.71 \pm 0.16$ & $0.75 \pm 0.19$ & GHS42 \\
\hline 132 & NPZ 213/08 & $0.71 \pm 0.30$ & $0.82 \pm 0.19$ & GHS43 \\
\hline 133 & SYN VER 239 & $0.72 \pm 0.28$ & $1.05 \pm 0.28$ & GHS42 \\
\hline 134 & NICK 199 & $0.72 \pm 0.27$ & $1.03 \pm 0.22$ & GHS46 \\
\hline 135 & SYN VER 265 & $0.72 \pm 0.31$ & $0.85 \pm 0.40$ & GHS46 \\
\hline 136 & SYN VER 247 & $0.73 \pm 0.33$ & $0.94 \pm 0.30$ & GHS43 \\
\hline 137 & NPZ 201/08 & $0.73 \pm 0.34$ & $0.76 \pm 0.31$ & GHS42 \\
\hline 138 & NPZ 209/08 & $0.73 \pm 0.15$ & $0.76 \pm 0.15$ & GHS42 \\
\hline 139 & SRG 214 & $0.73 \pm 0.15$ & $0.79 \pm 0.27$ & GHS43 \\
\hline 140 & WVB 224 & $0.74 \pm 0.25$ & $0.85 \pm 0.41$ & GHS43 \\
\hline 141 & SRG 208 & $0.74 \pm 0.27$ & $0.84 \pm 0.21$ & GHS42 \\
\hline 142 & WVB 215 & $0.75 \pm 0.30$ & $0.67 \pm 0.39$ & GHS43 \\
\hline 143 & SYN VER 230 & $0.75 \pm 0.29$ & $0.93 \pm 0.33$ & GHS42 \\
\hline 144 & SYN VER 228 & $0.75 \pm 0.21$ & $0.87 \pm 0.33$ & GHS42 \\
\hline 145 & KWS 196 & $0.75 \pm 0.13$ & $0.84 \pm 0.27$ & GHS42 \\
\hline 146 & NPZ 202/08 & $0.76 \pm 0.32$ & $0.82 \pm 0.35$ & GHS42 \\
\hline 147 & SYN VER 229 & $0.76 \pm 0.24$ & $0.97 \pm 0.14$ & GHS42 \\
\hline 148 & SRG 223 & $0.76 \pm 0.25$ & $0.63 \pm 0.28$ & GHS43 \\
\hline 149 & NPZ 214/08 & $0.76 \pm 0.23$ & $0.74 \pm 0.22$ & GHS43 \\
\hline 150 & SYN VER 252 & $0.76 \pm 0.70$ & $0.76 \pm 0.33$ & GHS44 \\
\hline 151 & SYN VER 236 & $0.77 \pm 0.16$ & $0.93 \pm 0.16$ & GHS42 \\
\hline 152 & SYN VER 242 & $0.77 \pm 0.17$ & $1.11 \pm 0.16$ & GHS43 \\
\hline 153 & SYN VER 254 & $0.79 \pm 0.28$ & $0.46 \pm 0.54$ & GHS44 \\
\hline 154 & SRG 224 & $0.79 \pm 0.88$ & $-0.21 \pm 0.86$ & GHS44 \\
\hline 155 & DSV $10-20$ & $0.79 \pm 0.32$ & $0.56 \pm 0.42$ & GHS43 \\
\hline 156 & SRG 215 & $0.79 \pm 0.29$ & $0.87 \pm 0.34$ & GHS43 \\
\hline 157 & WVB 216 & $0.79 \pm 0.24$ & $0.44 \pm 0.24$ & GHS43 \\
\hline 158 & WVB 218 & $0.81 \pm 0.24$ & $0.53 \pm 0.19$ & GHS43 \\
\hline 159 & SYN VER 258 & $0.81 \pm 0.74$ & $1.57 \pm 1.35$ & GHS44 \\
\hline 160 & SRG 203 & $0.81 \pm 0.39$ & $1.06 \pm 0.38$ & GHS42 \\
\hline
\end{tabular}


Appendix 2.3 continued

\begin{tabular}{|c|c|c|c|c|}
\hline No. & Genotype code & $\begin{array}{c}\text { Normalized net } \\
\text { AUDPC }\end{array}$ & $\begin{array}{l}\text { Normalized } \\
\text { relative stunting }\end{array}$ & $\begin{array}{c}\text { Experiment } \\
\text { No. }\end{array}$ \\
\hline 161 & DSV $10-22$ & $0.81 \pm 0.23$ & $0.93 \pm 0.25$ & GHS43 \\
\hline 162 & KWS 206 & $0.82 \pm 0.29$ & $0.89 \pm 0.27$ & GHS43 \\
\hline 163 & WVB 212 & $0.83 \pm 0.23$ & $0.89 \pm 0.30$ & GHS42 \\
\hline 164 & DSV 10-31 & $0.83 \pm 0.76$ & $0.79 \pm 0.61$ & GHS44 \\
\hline 165 & NPZ 206/08 & $0.83 \pm 0.38$ & $0.88 \pm 0.23$ & GHS42 \\
\hline 166 & SYN VER 263 & $0.84 \pm 0.27$ & $0.72 \pm 0.21$ & GHS46 \\
\hline 167 & KWS 217 & $0.85 \pm 0.26$ & $1.08 \pm 0.24$ & GHS46 \\
\hline 168 & WVB 219 & $0.85 \pm 0.43$ & $0.89 \pm 0.44$ & GHS43 \\
\hline 169 & SW 08-309031 & $0.85 \pm 0.59$ & $0.96 \pm 0.79$ & GHS44 \\
\hline 170 & SRG 229 & $0.85 \pm 0.94$ & $0.36 \pm 0.84$ & GHS44 \\
\hline 171 & WVB 206 & $0.86 \pm 0.34$ & $0.91 \pm 0.36$ & GHS42 \\
\hline 172 & WVB 217 & $0.87 \pm 0.30$ & $0.53 \pm 0.21$ & GHS43 \\
\hline 173 & DSV $10-18$ & $0.89 \pm 0.44$ & $0.84 \pm 0.58$ & GHS43 \\
\hline 174 & NICK 198 & $0.89 \pm 0.36$ & $1.11 \pm 0.32$ & GHS46 \\
\hline 175 & DSV 10-5 & $0.89 \pm 0.22$ & $1.23 \pm 0.17$ & GHS42 \\
\hline 176 & SYN VER 245 & $0.89 \pm 0.43$ & $1.02 \pm 0.40$ & GHS43 \\
\hline 177 & NPZ 210/08 & $0.90 \pm 0.24$ & $0.54 \pm 0.20$ & GHS43 \\
\hline 178 & NPZ 218/08 & $0.90 \pm 0.39$ & $0.85 \pm 0.35$ & GHS43 \\
\hline 179 & SYN VER 240 & $0.91 \pm 0.16$ & $1.18 \pm 0.23$ & GHS42 \\
\hline 180 & WVB 220 & $0.92 \pm 0.26$ & $0.09 \pm 0.14$ & GHS43 \\
\hline 181 & SRG 207 & $0.92 \pm 0.27$ & $1.16 \pm 0.37$ & GHS42 \\
\hline 182 & SW 12-5 & $0.94 \pm 0.42$ & $0.94 \pm 0.34$ & GHS46 \\
\hline 183 & SRG 206 & $0.94 \pm 0.31$ & $1.13 \pm 0.30$ & GHS42 \\
\hline 184 & NPZ 205/08 & $0.95 \pm 0.26$ & $1.00 \pm 0.31$ & GHS42 \\
\hline 185 & SW 12-2 & $0.96 \pm 0.52$ & $0.89 \pm 0.33$ & GHS46 \\
\hline 186 & KWS 190 & $0.97 \pm 0.28$ & $1.25 \pm 0.22$ & GHS42 \\
\hline 187 & NICK 185 & $0.97 \pm 0.67$ & $0.91 \pm 1.06$ & GHS44 \\
\hline 188 & WVB 229 & $0.97 \pm 1.00$ & $0.71 \pm 0.60$ & GHS44 \\
\hline 189 & NPZ 208/08 & $0.98 \pm 0.35$ & $1.22 \pm 0.24$ & GHS42 \\
\hline 190 & KWS 203 & $0.98 \pm 0.46$ & $0.96 \pm 0.42$ & GHS43 \\
\hline 191 & SRG 220 & $1.01 \pm 0.31$ & $0.96 \pm 0.36$ & GHS43 \\
\hline 192 & DSV $10-44$ & $1.02 \pm 0.42$ & $1.26 \pm 0.32$ & GHS46 \\
\hline 193 & KWS 193 & $1.02 \pm 0.23$ & $1.35 \pm 0.21$ & GHS42 \\
\hline 194 & KWS 220 & $1.03 \pm 0.49$ & $1.22 \pm 0.46$ & GHS46 \\
\hline 195 & SW 08-309032 & $1.03 \pm 0.72$ & $0.29 \pm 0.41$ & GHS44 \\
\hline 196 & NPZ 207/08 & $1.04 \pm 0.37$ & $1.16 \pm 0.32$ & GHS42 \\
\hline 197 & DSV $10-24$ & $1.04 \pm 0.33$ & $1.14 \pm 0.28$ & GHS43 \\
\hline 198 & DSV $10-25$ & $1.06 \pm 0.16$ & $1.10 \pm 0.25$ & GHS43 \\
\hline 199 & DSV $10-45$ & $1.06 \pm 0.27$ & $0.99+0.18$ & GHS46 \\
\hline 200 & WVB 221 & $1.07 \pm 0.31$ & $1.05 \pm 0.29$ & GHS43 \\
\hline
\end{tabular}


Appendix 2.3 continued

\begin{tabular}{|c|c|c|c|c|}
\hline No. & Genotype code & $\begin{array}{c}\text { Normalized net } \\
\text { AUDPC }\end{array}$ & $\begin{array}{l}\text { Normalized } \\
\text { relative stunting }\end{array}$ & Experiment No. \\
\hline 201 & DSV $10-43$ & $1.07 \pm 0.28$ & $1.18+0.34$ & GHS46 \\
\hline 202 & WVB 213 & $1.09 \pm 0.39$ & $1.10 \pm 0.31$ & GHS42 \\
\hline 203 & SRG 218 & $1.10 \pm 0.50$ & $1.11 \pm 0.36$ & GHS43 \\
\hline 204 & KWS 219 & $1.10 \pm 0.27$ & $0.94 \pm 0.22$ & GHS46 \\
\hline 205 & KWS 191 & $1.11 \pm 0.19$ & $1.27 \pm 0.16$ & GHS42 \\
\hline 206 & KWS 205 & $1.11 \pm 0.97$ & $0.99 \pm 0.13$ & GHS43 \\
\hline 207 & DSV $10-16$ & $1.12 \pm 0.40$ & $1.22 \pm 0.22$ & GHS42 \\
\hline 208 & SYN VER 259 & $1.12 \pm 1.24$ & $0.81 \pm 0.54$ & GHS44 \\
\hline 209 & SYN VER 249 & $1.13 \pm 0.31$ & $1.36 \pm 0.17$ & GHS43 \\
\hline 210 & DSV $10-19$ & $1.17 \pm 0.21$ & $1.00 \pm 0.26$ & GHS43 \\
\hline 211 & NICK 196 & $1.19 \pm 0.56$ & $1.17 \pm 0.43$ & GHS46 \\
\hline 212 & KWS 221 & $1.21 \pm 0.30$ & $1.37 \pm 0.22$ & GHS46 \\
\hline 213 & DSV $10-8$ & $1.21 \pm 0.29$ & $1.28 \pm 0.24$ & GHS42 \\
\hline 214 & NICK 183 & $1.22 \pm 0.30$ & $1.37 \pm 0.19$ & GHS42 \\
\hline 215 & SYN VER 235 & $1.23 \pm 0.29$ & $1.20 \pm 0.21$ & GHS42 \\
\hline 216 & WVB 222 & $1.23 \pm 0.30$ & $0.88 \pm 0.27$ & GHS43 \\
\hline 217 & SYN VER 257 & $1.24 \pm 1.03$ & $0.47 \pm 0.43$ & GHS44 \\
\hline 218 & KWS 199 & $1.25 \pm 0.29$ & $1.31 \pm 0.18$ & GHS42 \\
\hline 219 & DSV 10-21 & $1.27 \pm 0.27$ & $0.88 \pm 0.35$ & GHS43 \\
\hline 220 & DSV $10-15$ & $1.28 \pm 0.26$ & $1.37 \pm 0.13$ & GHS42 \\
\hline 221 & SYN VER 244 & $1.30 \pm 0.54$ & $1.24 \pm 0.54$ & GHS43 \\
\hline 222 & SYN VER 243 & $1.31 \pm 0.45$ & $1.34 \pm 0.37$ & GHS43 \\
\hline 223 & DSV 10-41 & $1.32 \pm 0.43$ & $1.59 \pm 0.34$ & GHS46 \\
\hline 224 & WVB 229 & $1.32 \pm 0.25$ & $0.99 \pm 0.18$ & GHS46 \\
\hline 225 & KWS 222 & $1.34 \pm 0.25$ & $1.21 \pm 0.31$ & GHS46 \\
\hline 226 & WVB 226 & $1.34 \pm 0.29$ & $1.07 \pm 0.36$ & GHS46 \\
\hline 227 & KWS 192 & $1.37 \pm 0.25$ & $1.33 \pm 0.16$ & GHS42 \\
\hline 228 & DSV $10-42$ & $1.37 \pm 0.43$ & $1.58 \pm 0.32$ & GHS46 \\
\hline 229 & Falcon & $1.42 \pm 0.31$ & $1.47 \pm 0.21$ & Susceptible standard \\
\hline 230 & DSV $10-14$ & $1.43 \pm 0.41$ & $1.38 \pm 0.24$ & GHS42 \\
\hline 231 & SYN VER 234 & $1.46 \pm 0.22$ & $1.43 \pm 0.09$ & GHS43 \\
\hline 232 & SYN VER 241 & $1.61 \pm 0.65$ & $1.39 \pm 0.29$ & GHS43 \\
\hline 233 & KWS 218 & $1.70 \pm 0.43$ & $1.43 \pm 0.58$ & GHS46 \\
\hline 234 & SYN VER 255 & $1.71 \pm 1.66$ & $0.70 \pm 0.58$ & GHS44 \\
\hline 235 & SYN VER 253 & $2.11 \pm 1.01$ & $-0.03 \pm 0.63$ & GHS44 \\
\hline 236 & WVB 223 & $2.22 \pm 0.30$ & $1.66 \pm 0.05$ & GHS43 \\
\hline 237 & KWS 204 & $2.35 \pm 0.47$ & $1.59 \pm 0.21$ & GHS43 \\
\hline
\end{tabular}

Mean \pm standard deviation data obtained from 20 plants of 235 Brassica napus lines evaluated in four different experiments are shown. Genotypes are sorted according to increasing values of normalized net AUDPC results. The values shown for resistant (Express; No. 92) and susceptible (Falcon; No.229) controls are indicated in bold. For the reference varieties, mean values obtained from four independent experiments (GHS42, GHS43, GHS44 and GHS46) are shown. Genotypes marked with * are best performing lines recommended for further verification under field conditions. 
Appendix 2.4 Monthly air temperature, relative humidity and rainfall at the outdoor experimental area.

\begin{tabular}{cccc} 
Month & Total RF $(\mathrm{mm})$ & $\mathrm{T}\left({ }^{\circ} \mathrm{C}\right)$ & $\mathrm{RH}(\%)$ \\
\hline April 2012 & 21.2 & 8.6 & 70.1 \\
May 2012 & 31.4 & 15.2 & 69.0 \\
June 2012 & 97.6 & 15.3 & 78.6 \\
July 2012 & 90.9 & 18.0 & 80.7 \\
\hline
\end{tabular}

$\mathbf{R F}$, rain fall. $\mathbf{T}$, temperature. $\mathbf{R H}$, relative humidity. 
Appendix 2.5 Comparison of branching in control and infected Brassica napus double haploid lines screened for Verticillium longisporum resistance in the outdoor experiment.

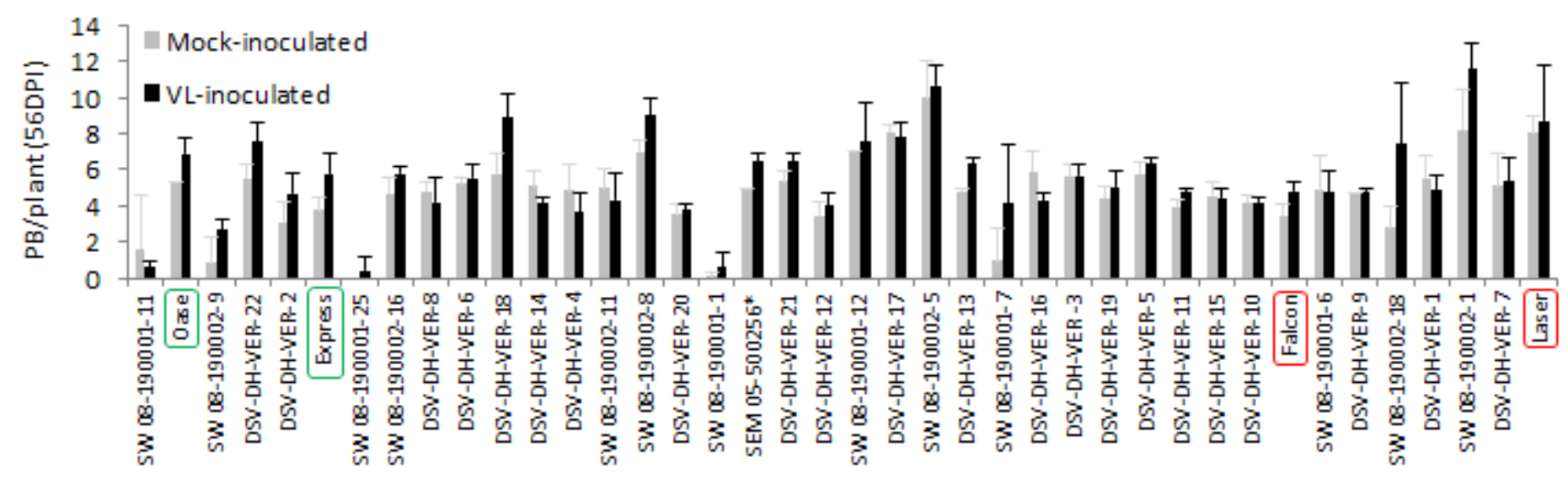

Brassica napus lines

PB, primary branches. VL, Verticillium longisporum. ${ }^{*}$, not a double haploid line. Mean \pm standard deviation data obtained from three biological replicates is shown. Values of resistant and susceptible controls are indicated in green and red marks, respectively. Genotypes are sorted according to increasing net AUDPC values. 
Appendix 2.6 Assessment key for evaluation of Verticillium longisporum disease severity on stubble and root of Brassica napus.

\begin{tabular}{|c|c|c|}
\hline Index & Disease category & Symptom descriptions \\
\hline 1 & Healthy tissue & $\begin{array}{l}\text { Surface free of any symptoms and fungal structures; pith white } \\
\text { and with cottony appearance; no microsclerotia visible }\end{array}$ \\
\hline 2 & Slight infestation & $\begin{array}{l}\text { Narrow and short longitudinal lesions on the surface; pith still } \\
\text { whitish but a few patches of microsclerotia visible }\end{array}$ \\
\hline 3 & $\begin{array}{l}\text { Advanced } \\
\text { infestation }\end{array}$ & $\begin{array}{l}\text { Very long lesions and abundant microsclerotia visible under the } \\
\text { epidermis; epidermis peeling off; long patches of black } \\
\text { microsclerotia visible in the pith }\end{array}$ \\
\hline 4 & Severe infestation & $\begin{array}{l}\text { Most part of the stem epidermis peeling off and disintegrated; } \\
\text { the surface black and covered with microsclerotia; pith becomes } \\
\text { dark, disintegrated and filled with microsclerotia }\end{array}$ \\
\hline
\end{tabular}


Appendix 3.1 Standard curve for quantification of total carbohydrate content in xylem sap.

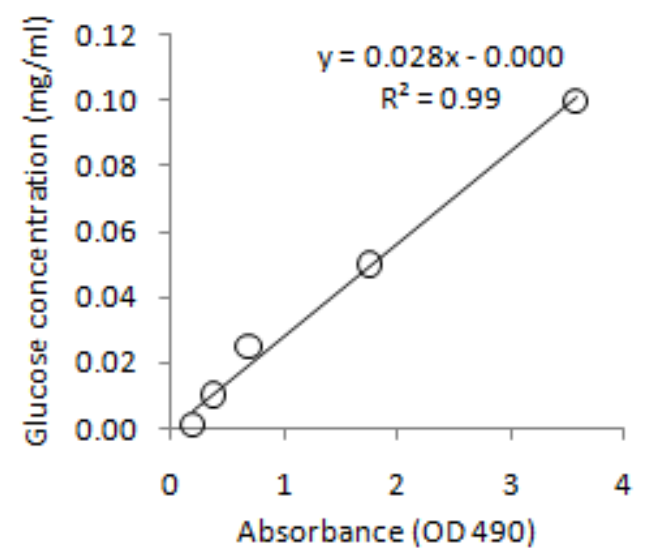

Points represent means of triplicates. Bovine serum albumin (BSA) was used as protein standard. 
Appendix 3.2 Standard curve for quantification of total protein concentration in xylem sap.

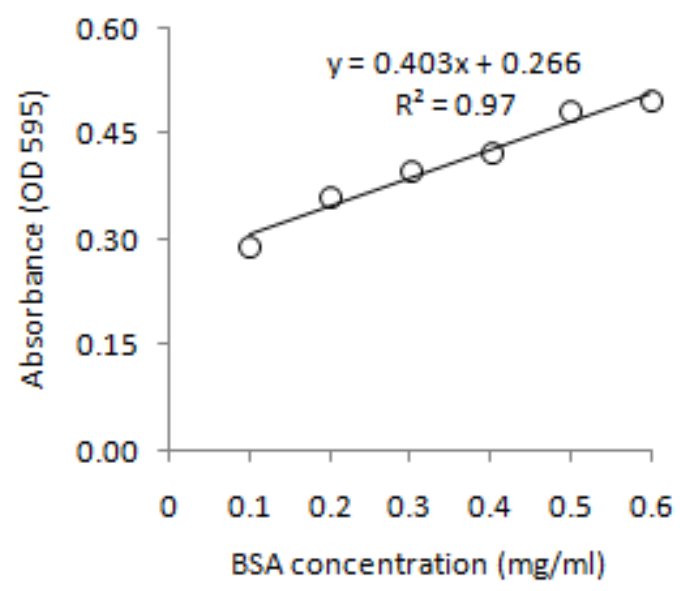

Points represent means of triplicates. Bovine serum albumin (BSA) was used as protein standard. 
Appendix 3.3 Measurement of Verticillium longisporum growth in xylem sap of resistant (SEM) and susceptible (Falcon) Brassica napus plants. Xylem sap was collected 28 days after inoculation with Verticillium longisporum.

\begin{tabular}{|c|c|c|c|c|c|c|}
\hline \multirow[b]{2}{*}{ Treatments } & \multicolumn{6}{|c|}{ Fungal growth on xylem sap (OD 580) } \\
\hline & $2 \mathrm{DAl}$ & $3 \mathrm{DAl}$ & $4 \mathrm{DAl}$ & $5 \mathrm{DAl}$ & $6 \mathrm{DAl}$ & $7 \mathrm{DAl}$ \\
\hline FM & $0.044 \pm 0.02^{\mathrm{ab}}$ & $0.195 \pm 0.07^{a}$ & $0.223 \pm 0.06^{a}$ & $0.317 \pm 0.09^{a b}$ & $0.358 \pm 0.09^{a b}$ & $0.407 \pm 0.12^{a}$ \\
\hline FMf & $0.029 \pm 0.02^{b}$ & $0.213 \pm 0.06^{a}$ & $0.254 \pm 0.04^{\mathrm{a}}$ & $0.363 \pm 0.07^{a b}$ & $0.395 \pm 0.06^{a b}$ & $0.448+0.05^{a}$ \\
\hline FVL & $0.086 \pm 0.06^{a b}$ & $0.291 \pm 0.22^{a}$ & $0.320 \pm 0.26^{a}$ & $0.441 \pm 0.34^{a b}$ & $0.465 \pm 0.35^{a b}$ & $0.489 \pm 0.34^{\mathrm{a}}$ \\
\hline FVLf & $0.028 \pm 0.00^{b}$ & $0.181 \pm 0.03^{a}$ & $0.192 \pm 0.02^{a}$ & $0.273 \pm 0.04^{b}$ & $0.295 \pm 0.06^{b}$ & $0.350 \pm 0.10^{\mathrm{a}}$ \\
\hline SM & $0.045 \pm 0.03^{b}$ & $0.182 \pm 0.04^{a}$ & $0.243 \pm 0.10^{a}$ & $0.359 \pm 0.12^{a b}$ & $0.435 \pm 0.14^{a b}$ & $0.548 \pm 0.21^{\mathrm{a}}$ \\
\hline SMf & $0.038 \pm 0.02^{b}$ & $0.208 \pm 0.02^{a}$ & $0.270 \pm 0.01^{a}$ & $0.386 \pm 0.04^{a b}$ & $0.443 \pm 0.03^{a b}$ & $0.508 \pm 0.03^{a}$ \\
\hline SVL & $0.114 \pm 0.05^{a}$ & $0.383 \pm 0.16^{a}$ & $0.418 \pm 0.17^{a}$ & $0.515 \pm 0.19^{a}$ & $0.585 \pm 0.22^{a}$ & $0.611 \pm 0.23^{\mathrm{a}}$ \\
\hline SVLf & $0.039 \pm 0.02^{b}$ & $0.228 \pm 0.03^{a}$ & $0.291 \pm 0.04^{\mathrm{a}}$ & $0.430 \pm 0.08^{a b}$ & $0.493 \pm 0.13^{a b}$ & $0.573 \pm 0.17^{a}$ \\
\hline BDWVL & $0.009 \pm 0.00^{c}$ & $0.020 \pm 0.02^{a}$ & $0.017 \pm 0.00^{\mathrm{a}}$ & $0.038 \pm 0.03^{c}$ & $0.036 \pm 0.00^{c}$ & $0.018 \pm 0.00^{\mathrm{a}}$ \\
\hline BDW & $0.007 \pm 0.00^{c}$ & $0.010 \pm 0.00^{a}$ & $0.009 \pm 0.00^{a}$ & $0.037 \pm 0.01^{c}$ & $0.019 \pm 0.00^{c}$ & $0.009 \pm 0.00^{a}$ \\
\hline
\end{tabular}

SM and SMf, respective fungal growth in unfiltered and filtered xylem sap collected from mockinoculated plants. SVL and SVLf, respective fungal growth in unfiltered and filtered xylem sap extracted from VL-inoculated plants. FM and FMf, respective fungal growth in unfiltered and filtered xylem sap of mock-inoculated plants. SVL and SVLf, respective fungal growth in unfiltered and filtered xylem sap of VL-inoculated plants. BDWVL, Fungal growth in sterile bi-distilled water. BDW, sterile bi-distilled water without VL inoculum. Means \pm standard deviation were obtained from three biological replicates. Mean values assigned with the same superscript are not significantly different at $P=0.05$. 
Appendix 4.1 Transpiration rate (E), Stomatal conductance of $\mathrm{CO}_{2}(\mathrm{gs})$, photosynthesis rate (A), and water use efficiency (WUE) of two Brassica napus genotypes subjected to Verticillium longisporum infection and/or drought stress under greenhouse conditions at $28 \mathrm{DPI}$.
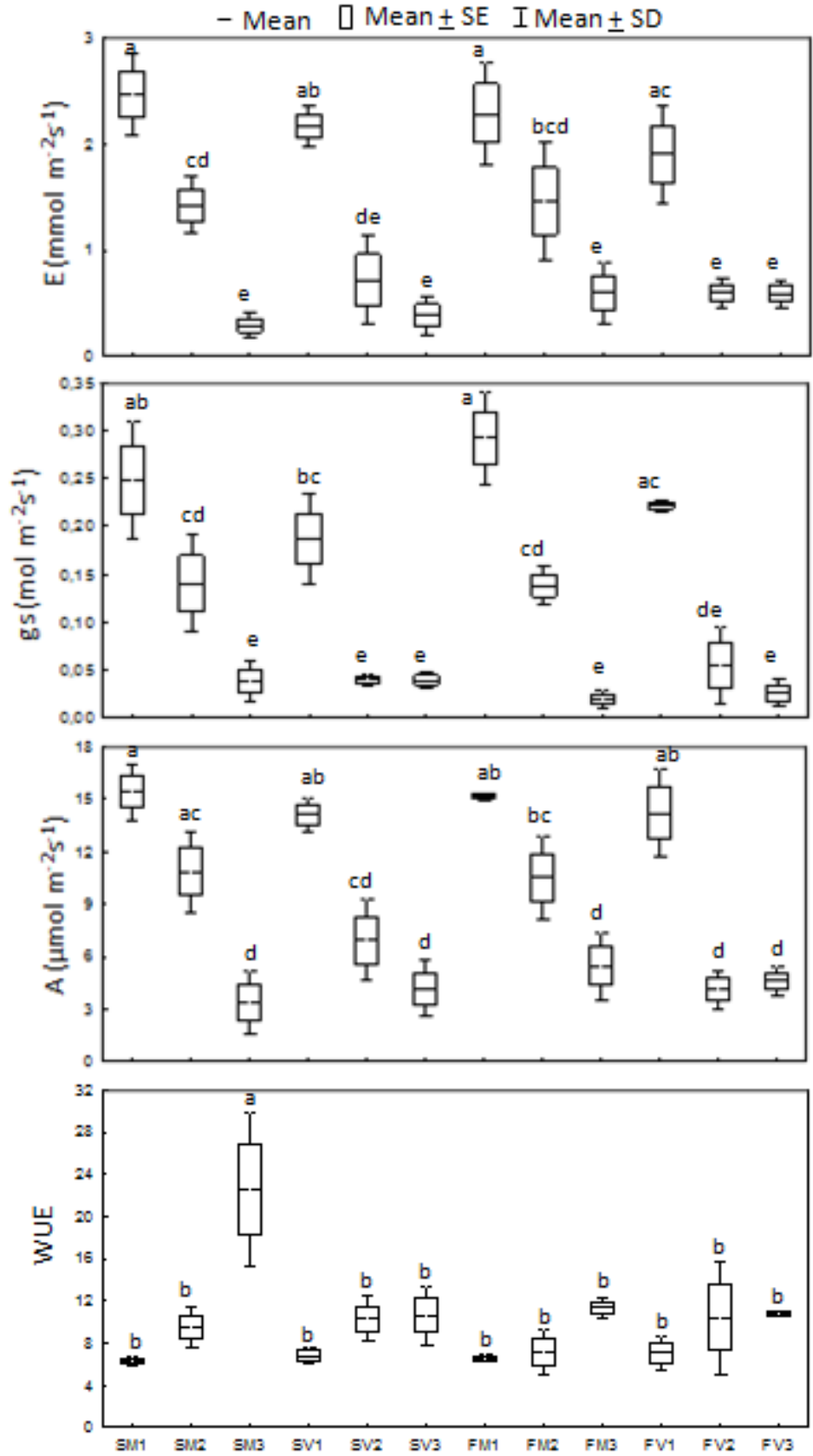

Mock and/or VL-inoculated plants supplied with water at $100 \%$ FC were used as control. Drought treatments were made by exposing plants to moderate $(60 \% \mathrm{FC})$ or severe $(30 \% \mathrm{FC})$ drought stress for one week starting from 21 days after inoculation with Verticillium longisporum. Mean data obtained from 30 plants of two independent experiments are presented. For each parameter, different letters on the bars indicate significant differences between treatments at $\mathrm{P} \leq 0.05$. SM, SEM-05-500526 mock-inoculated. FM, Falcon mock-inoculated. SV, SEM-05500526 VL-inoculated. FV, Falcon VL-inoculated. Treatment names followed by the numbers 1, 2 and $\mathbf{3}$ refers to watering at 100, 60 and $30 \%$ field capacity, respectively. 
Appendix 4.2 Changes in proline content in leaf and hypocotyl tissue of two Brassica napus genotypes exposed to different levels of drought stress and Verticillium longisporum infection at 28 DPI.

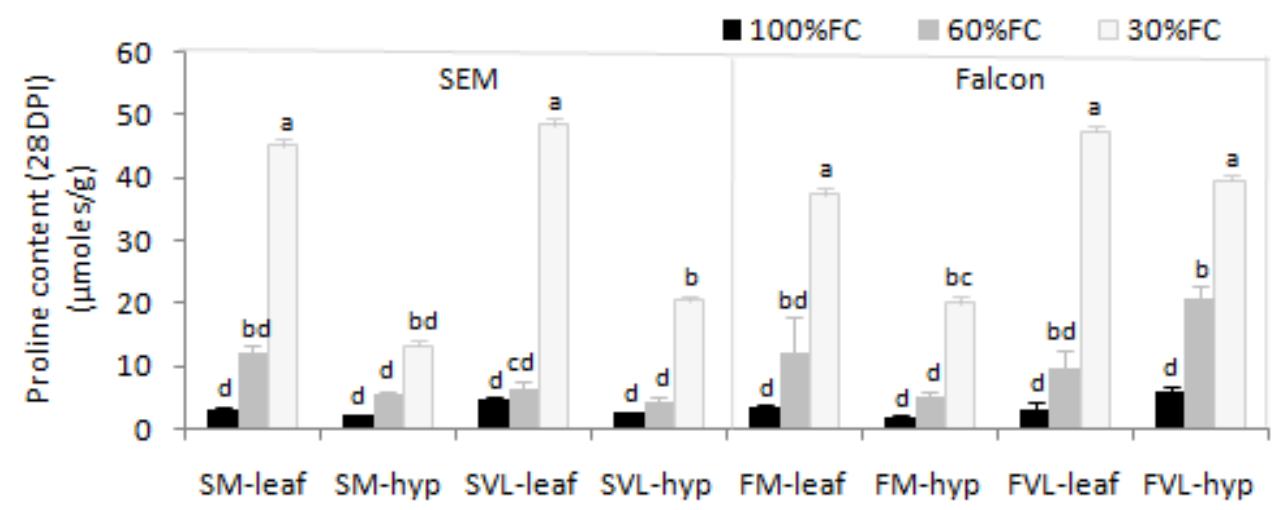

Drought treatments were made by exposing plants to moderate (60\% FC) or severe (30\% FC) drought stress for one week starting from 21 days after inoculation with Verticillium longisporum. Mock and/or VL-inoculated plants supplied with water at $100 \%$ FC were used as control. Mean data obtained from 30 plants of two independent experiments are presented. Bars indicate standard deviations. Different letters on the bars indicate significant differences between treatments at $P \leq 0.05$. FC, field capacity. hyp, hypocotyl. SM, SEM-05-500526 mockinoculated. FM, Falcon mock-inoculated. SVL, SEM-05-500526 VL-inoculated. FVL, Falcon VL-inoculated. 
Appendix 4.3 Number of primary branches per plant measured from two Brassica napus genotypes exposed to drought stress and/or Verticillium longisporum infection at $28 \mathrm{DPI}$.

\begin{tabular}{cccc} 
Treatments & $100 \% \mathrm{FC}$ & $60 \% \mathrm{FC}$ & $30 \% \mathrm{FC}$ \\
\hline SM & $1.8 \pm 0.4^{\mathrm{bc}}$ & $1.9 \pm 0.7^{\mathrm{bc}}$ & $1.9 \pm 0.7^{\mathrm{bc}}$ \\
SVL & $4.2 \pm 1.0^{\mathrm{ab}}$ & $3.9 \pm 1.4^{\mathrm{ab}}$ & $4.2 \pm 1.3^{\mathrm{ab}}$ \\
FM & $0.6 \pm 0.2^{\mathrm{c}}$ & $0.5 \pm 0.2^{\mathrm{c}}$ & $0.8 \pm 0.4^{\mathrm{c}}$ \\
FVL & $3.8 \pm 0.6^{\mathrm{ab}}$ & $5.1 \pm 1.8^{\mathrm{a}}$ & $4.9 \pm 1.2^{\mathrm{a}}$
\end{tabular}

Drought treatments were made by exposing plants to moderate (60\% FC) or severe ( $30 \%$ FC) drought stress for one week starting from 21 days after inoculation with Verticillium longisporum. Mock and/or VL-inoculated plants supplied with water at $100 \%$ FC were used as control. Mean \pm Standard deviation data obtained from 30 plants of two independent experiments are presented. Mean values containing the same superscript are not significantly different at $P=0.05$. FC, field capacity. SM, SEM-05-500526 mock-inoculated. FM, Falcon mock-inoculated. SVL, SEM-05-500526 VL-inoculated. FVL, Falcon VL-inoculated. 
Appendix 4.4 Average phenological growth stages of two Brassica napus genotypes exposed to drought stress and/or Verticillium longisporum infection.

\begin{tabular}{cccccc}
\hline Treatments & 21 DPI & 28 DPI & 35 DPI & 42 DPI & 49 DPI \\
\hline SM1 & 50 & 59 & 62 & 65 & 67 \\
SM2 & 50 & 58 & 63 & 65 & 68 \\
SM3 & 48 & 55 & 60 & 60 & 63 \\
SV1 & 49 & 56 & 60 & 63 & 65 \\
SV2 & 52 & 59 & 62 & 64 & 66 \\
SV3 & 47 & 54 & 57 & 59 & 60 \\
FM1 & 47 & 51 & 56 & 59 & 62 \\
FM2 & 45 & 51 & 56 & 58 & 60 \\
FM3 & 46 & 51 & 56 & 58 & 59 \\
FV1 & 43 & 49 & 53 & 57 & 61 \\
FV2 & 44 & 50 & 54 & 57 & 59 \\
FV3 & 45 & 50 & 56 & 58 & 62
\end{tabular}

Drought treatments were made by exposing plants to moderate $(60 \% \mathrm{FC})$ or severe $(30 \% \mathrm{FC})$ drought stress for four weeks starting from 21 days after inoculation with Verticillium longisporum. Mock and/or VL-inoculated plants supplied with water at $100 \%$ FC were used as control. Mean data obtained from 30 plants of two independent experiments are presented. FC, field capacity. S, genotype SEM-05-500526. F, cultivar Falcon. M, mock-inoculated. V, infected with Verticillium longisporum. 1, 2 and $\mathbf{3}$ refers to watering at 100, 60 and 30\% field capacity, respectively. Assessment was done using $\mathrm{BBCH}$ scale. 
Appendix 4.5 Shoot and root dry biomass yields of two Brassica napus genotypes subjected to Verticillium longisporum infection and/or drought stress conditions in a greenhouse experiment at $28 \mathrm{DPI}$.

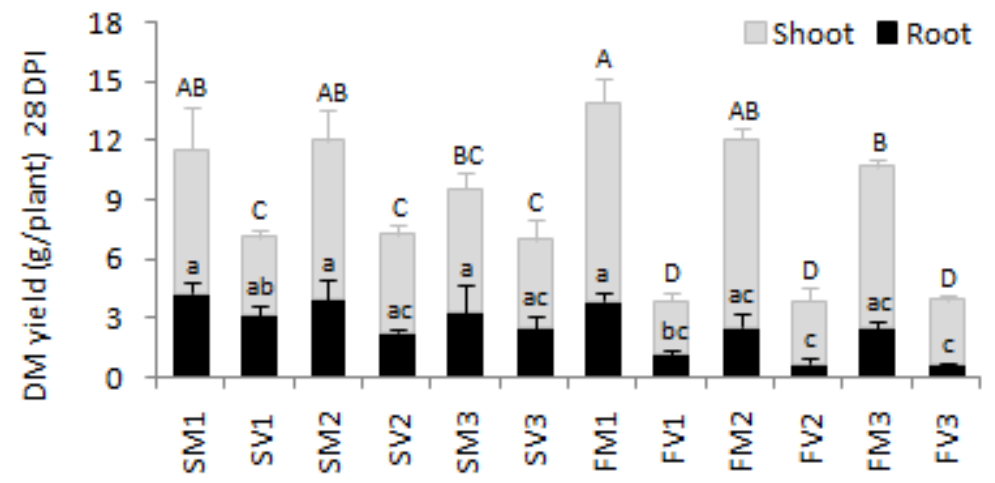

Mock and/or VL-inoculated plants supplied with water at $100 \% \mathrm{FC}$ were used as control. Drought treatments were made by exposing plants to moderate $(60 \% \mathrm{FC})$ or severe $(30 \% \mathrm{FC})$ drought stress for one week starting from 21 days after inoculation with Verticillium longisporum. Mean data obtained from 30 plants of two independent experiments are presented. Bars indicate standard deviation. For each parameter, different letters on the bars with the same letter-case indicate significant differences between treatments at $\mathbf{P} \leq 0.05$. DM, dry matter. $\mathbf{S}$, genotype SEM-05-500526. F, cultivar Falcon. M, mock-inoculated. V, infected with Verticillium longisporum. Treatments names followed by numbers 1, 2 and $\mathbf{3}$ refer to watering at 100, 60 and 30\% field capacity, respectively. 
Appendix 4.6 Total dry biomass yield of two Brassica napus genotypes subjected to Verticillium longisporum infection and/or drought stress in a greenhouse experiment at $28 \mathrm{DPI}$.

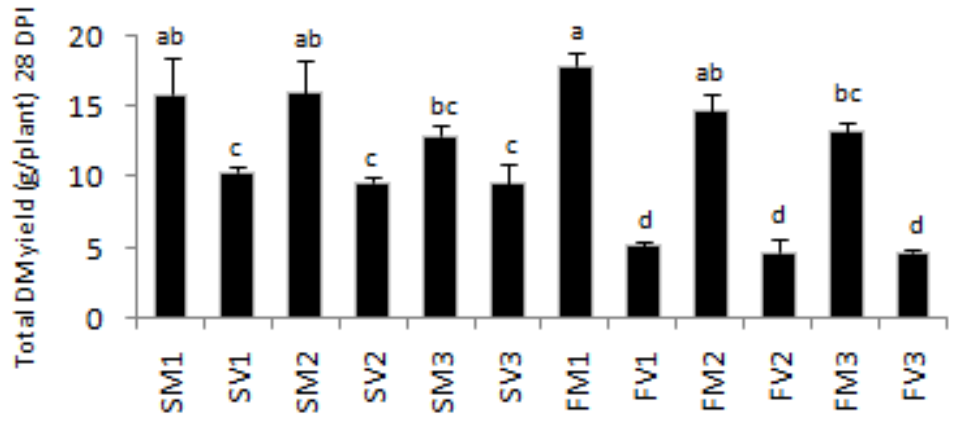

Mock- and/or VL-inoculated plants supplied with water at $100 \%$ FC were used as control. Drought treatments were made by exposing plants to moderate $(60 \% \mathrm{FC})$ or severe $(30 \% \mathrm{FC})$ drought stress for one week starting from 21 days after inoculation with Verticillium longisporum. Mean data obtained from 30 plants of two independent experiments are presented. Bars indicate standard deviation. For each parameter, different letters on the bars indicate significant difference between treatments at $P \leq 0.05$. S, genotype SEM-05-500526. F, cultivar Falcon. $\mathbf{M}$, mock inoculated. $\mathbf{V}$, infected with Verticillium longisporum. Treatments names followed by numbers $\mathbf{1}, \mathbf{2}$ and $\mathbf{3}$ refer to watering at 100, 60 and $30 \%$ field capacity, respectively. 
Appendix 4.7 F-values of analysis of variance for the effects of genotype, Verticillium longisporum infection, drought stress and interaction factors on disease development, plant physiolgy and agronomic trtaits measured at 28 DPI.

\begin{tabular}{|c|c|c|c|c|c|c|c|c|}
\hline \multicolumn{2}{|c|}{ Parameters } & G & VL & $D$ & G*VL & $G * D$ & $V L * D$ & $G * V L * D$ \\
\hline \multirow{5}{*}{ 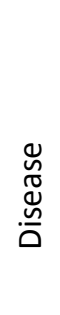 } & DF & 1 & 1 & 2 & 1 & 2 & 2 & 2 \\
\hline & AUDPC & $103.6^{* * *}$ & $650.3^{* * *}$ & $5.2^{*}$ & $103.6^{* * *}$ & $4.0^{*}$ & $5.2^{*}$ & $4.0^{*}$ \\
\hline & VL DNA & $183.3^{* * *}$ & $224.7^{* * *}$ & $2.3^{\mathrm{ns}}$ & $183.3^{* * *}$ & $2.4^{\mathrm{ns}}$ & $2.3^{\mathrm{ns}}$ & $2.4^{\mathrm{ns}}$ \\
\hline & RS & $31.0^{*}$ & $491.1^{* * *}$ & $1.9^{\mathrm{ns}}$ & $41.7^{* *}$ & $3.9^{*}$ & $1.9^{\mathrm{ns}}$ & $3.9^{*}$ \\
\hline & PB & $1.6^{\mathrm{ns}}$ & $98.8^{* * *}$ & $0.8^{\mathrm{ns}}$ & $7.4^{*}$ & $0.9^{\mathrm{ns}}$ & $0.5^{\mathrm{ns}}$ & $1.0^{\mathrm{ns}}$ \\
\hline \multirow{5}{*}{ 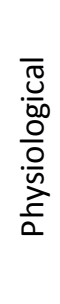 } & $E$ & $0.0^{\mathrm{ns}}$ & $19.0^{* * *}$ & $151.6^{* * *}$ & $0.5^{\mathrm{ns}}$ & $2.8^{\mathrm{ns}}$ & $8.3^{* *}$ & $0.0^{\mathrm{ns}}$ \\
\hline & gs & $0.9^{\mathrm{ns}}$ & $24.6^{* * *}$ & $139.3^{* * *}$ & $0.0^{\mathrm{ns}}$ & $2.3^{\mathrm{ns}}$ & $7.6^{* *}$ & $0.1^{\mathrm{ns}}$ \\
\hline & A & $0.1^{\mathrm{ns}}$ & $16.1^{* *}$ & $133.2^{* * *}$ & $1.4^{\mathrm{ns}}$ & $2.4^{\mathrm{ns}}$ & $8.9^{* *}$ & $0.7^{\mathrm{ns}}$ \\
\hline & WUE & $5.2^{*}$ & $1.7^{\mathrm{ns}}$ & $19.8^{* * *}$ & $6.1^{*}$ & $3.5^{*}$ & $7.4^{* *}$ & $3.4^{\mathrm{ns}}$ \\
\hline & RWC & $0.2^{\mathrm{ns}}$ & $0.9^{\mathrm{ns}}$ & $23.1^{* * *}$ & $2.2^{\mathrm{ns}}$ & $0.4^{\mathrm{ns}}$ & $0.9^{\mathrm{ns}}$ & $0.9^{\text {ns }}$ \\
\hline \multirow{4}{*}{ 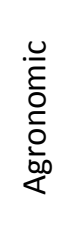 } & $\mathrm{PH}$ & $4.7^{\mathrm{ns}}$ & $87.9^{* * *}$ & $1.6^{\mathrm{ns}}$ & $24.6^{* * *}$ & $0.0^{\mathrm{ns}}$ & $5.2^{*}$ & $2.5^{\mathrm{ns}}$ \\
\hline & SDM & $9.4^{* *}$ & $339.8^{* * *}$ & $5.8^{* *}$ & $44.5^{* * *}$ & $1.2^{\mathrm{ns}}$ & $5.2^{*}$ & $0.9^{\text {ns }}$ \\
\hline & RDM & $34.6^{* * *}$ & $53.9^{* * *}$ & $6.2^{* *}$ & $4.2^{\mathrm{ns}}$ & $0.2^{\mathrm{ns}}$ & $0.7^{\mathrm{ns}}$ & $0.8^{\mathrm{ns}}$ \\
\hline & TDM & $28.7^{* * *}$ & $319.0^{* * *}$ & $8.7^{* *}$ & $37.9^{* * *}$ & $1.1^{\mathrm{ns}}$ & $4.7^{*}$ & $1.4^{\mathrm{ns}}$ \\
\hline
\end{tabular}

Drought treatments were applied by exposing plants to moderate $(60 \% \mathrm{FC})$ or severe $(30 \% \mathrm{FC})$ drought stress for one week starting from 21 days after inoculation with Verticillium longisporum ( 28 days post inoculation). ANOVA was performed using the PROC MIXED procedure (SAS 9.3, SAS Inst. 2002). P-values are indicated in superscripts and the values less than 0.05 are considered a significant effect. ${ }^{*}$, significant at $P=0.05$. ${ }^{* *}$, significant at $P=0.01$. ***, significant at $P=0.001$. ns, not significant. DF, degree of freedom. $\mathbf{G}$, genotype. VL, Verticillium longisporum. $\mathbf{D}$, drought. AUDPC, net area under disease progress curve, VL DNA, Verticillium longisporum DNA in hypocotyl. RS, relative stunting. PB, number of primary branches per plant. E, transpiration rate. gs, stomatal conductance of $\mathrm{CO}_{2}$. A, photosynthesis rate. WUE, water use efficiency. LPC, leeaf proline content. HPC, hypocotyl proline content. RWC, leaf relative water content. PH, plant height. SDM, shoot dry matter. RDM, root dry matter. TDM, total dry matter. 
Appendix 4.8 Analysis of variance for the effects of genotype, Verticillium longisporum infection, drought stress, and interaction factors on accumulation of free proline in leaf and hypocotyl tissue of Brassica napus at $28 \mathrm{DPI}$.

\begin{tabular}{lll}
\multicolumn{1}{c}{ Factors } & F-value & DF \\
\hline G & $8.5^{*}$ & 1 \\
VL & $15.2^{* *}$ & 1 \\
D & $306.0^{* * *}$ & 2 \\
PP & $78.4^{* * *}$ & 1 \\
G*VL & $9.2^{*}$ & 1 \\
G*D & $1.6^{\text {ns }}$ & 2 \\
G*PP & $25.4^{* * *}$ & 1 \\
VL*D & $7.0^{* *}$ & 2 \\
VL*PP & $14.1^{* *}$ & 1 \\
D*PP & $65.0^{* * *}$ & 2 \\
G*VLD & $2.0^{\text {ns }}$ & 2 \\
G*VLPP & $6.1^{*}$ & 1 \\
G*D*PP & $7.0^{* *}$ & 2 \\
VL*D*PP & $2.9^{\text {ns }}$ & 2 \\
G*VL D*PP & $0.7^{\text {ns }}$ & 2 \\
\hline$-{ }^{*}$ &
\end{tabular}

Drought treatments were applied by exposing plants to moderate $(60 \% \mathrm{FC})$ or severe $(30 \% \mathrm{FC})$ drought stress for one week starting from 21 days after inoculation with Verticillium longisporum ( 28 days post inoculation). ANOVA was performed using the PROC MIXED procedure (SAS 9.3, SAS Inst. 2002). P-values are indicated in superscripts and the values less than 0.05 are considered a significant effect. ${ }^{*}$, significant at $P=0.05$. ${ }^{* *}$, significant at $P=0.01$. $* * *$, significant at $P=0.001$. ns, not significant. DF, degree of freedom. $\mathbf{G}$, genotype. VL, Verticillium longisporum. $\mathbf{D}$, drought. PP, plant part. 
Appendix 4.9 Coefficients of Pearson's correlation ( $r$ ) describing relationships within and between disease, physiological and agronomic parameters measured from two Brassica napus genotypes exposed to drought stress and/or Verticillium longisporum infection 7 days after initiation of drought treatments (28 DPI).

\begin{tabular}{|c|c|c|c|c|c|c|c|c|c|c|c|c|c|c|c|}
\hline & & \multicolumn{4}{|c|}{ Disease } & \multicolumn{7}{|c|}{ Physiological } & \multicolumn{3}{|c|}{ Agronomic } \\
\hline \multicolumn{2}{|c|}{ Parameters } & AUDPC & VL DNA & $\mathrm{RS}$ & PB & $E$ & gs & $A$ & WUE & RWC & LPC & $\mathrm{HPC}$ & $\mathrm{PH}$ & SDM & RDM \\
\hline \multirow{3}{*}{ 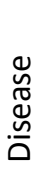 } & VL DNA & $0.87^{* * *}$ & $0.82^{* * *}$ & & & & & & & & & & & & \\
\hline & $\mathrm{RS}$ & $0.97^{* * *}$ & $0.63^{* * *}$ & & & & & & & & & & & & \\
\hline & PB & $0.76^{* * *}$ & $-0.21^{\mathrm{ns}}$ & $0.84^{* * *}$ & & & & & & & & & & & \\
\hline \multirow{7}{*}{$\begin{array}{l}\overline{\frac{J}{0}} \\
\frac{0}{00} \\
\frac{0}{N} \\
\frac{c}{\alpha}\end{array}$} & $E$ & $-0.20^{\mathrm{ns}}$ & $-0.18^{\mathrm{ns}}$ & $-0.17^{\mathrm{ns}}$ & $-0.26^{\mathrm{ns}}$ & & & & & & & & & & \\
\hline & gs & $-0.19^{\mathrm{ns}}$ & $-0.24^{\mathrm{ns}}$ & $-0.18^{\mathrm{ns}}$ & $-0.27^{\mathrm{ns}}$ & $0.92^{* * *}$ & & & & & & & & & \\
\hline & $A$ & $-0.21^{\mathrm{ns}}$ & $-0.02^{\mathrm{ns}}$ & $0.18^{\mathrm{ns}}$ & $-0.27^{\mathrm{ns}}$ & $0.96^{* * *}$ & $0.91^{* * *}$ & & & & & & & & \\
\hline & WUE & $-0.11^{\mathrm{ns}}$ & $-0.18^{\mathrm{ns}}$ & $-0.12^{\mathrm{ns}}$ & $0.05^{\mathrm{ns}}$ & $-0.54^{* * *}$ & $-0.50^{* *}$ & $-0.55^{* * *}$ & & & & & & & \\
\hline & RWC & $-0.15^{\mathrm{ns}}$ & $-0.11^{\mathrm{ns}}$ & $0.14^{\mathrm{ns}}$ & $-0.17^{\mathrm{ns}}$ & $0.58^{* * *}$ & $0.63^{* * *}$ & $0.63^{* * *}$ & $0.35^{*}$ & & & & & & \\
\hline & LPC & $-0.02^{\mathrm{ns}}$ & $0.59^{* * *}$ & $0.01^{\mathrm{ns}}$ & $0.10^{\mathrm{ns}}$ & $-0.72^{* * *}$ & $-0.70^{* * *}$ & $-0.74^{* * *}$ & $0.58^{* * *}$ & $-0.53^{* *}$ & & & & & \\
\hline & $\mathrm{HPC}$ & $-0.43^{* *}$ & $-0.77^{* * *}$ & $0.43^{* *}$ & $0.40^{*}$ & $-0.63^{* * *}$ & $-0.62^{* * *}$ & $-0.68^{* * *}$ & $0.32^{*}$ & $-0.52^{* *}$ & $0.73^{* * *}$ & & & & \\
\hline \multirow{4}{*}{$\begin{array}{l}. \frac{U}{\varepsilon} \\
\frac{0}{c} \\
\frac{\partial}{0} \\
\frac{0}{\alpha 0}\end{array}$} & $\mathrm{PH}$ & $-0.85^{* * *}$ & $-0.77^{* * *}$ & $-0.87^{* * *}$ & $-0.71^{* * *}$ & $0.30^{*}$ & $0.29^{*}$ & $0.31^{*}$ & $-0.11^{\mathrm{ns}}$ & $0.24^{\mathrm{ns}}$ & $-0.14^{\mathrm{ns}}$ & $0.48^{* *}$ & & & \\
\hline & SDM & $-0.91^{* * *}$ & $-0.76^{* * *}$ & $-0.92^{* * *}$ & $-0.83^{* * *}$ & $0.35^{*}$ & $0.36^{*}$ & $0.33^{*}$ & $-0.06^{\mathrm{ns}}$ & $0.21^{\mathrm{ns}}$ & $-0.18^{\mathrm{ns}}$ & $-0.49^{* *}$ & $0.83^{* * *}$ & & \\
\hline & RDM & $-0.79^{* * *}$ & $-0.80^{* * *}$ & $-0.74^{* * *}$ & $-0.56^{* * *}$ & $0.43^{* *}$ & $0.43^{* *}$ & $0.44^{* *}$ & $-0.01^{\mathrm{ns}}$ & $0.35^{*}$ & $-0.22^{\mathrm{ns}}$ & $-0.54^{* * *}$ & $0.69^{* * *}$ & $0.77^{* * *}$ & \\
\hline & TDM & $-0.92^{* * *}$ & $0.82^{* * *}$ & $-0.91^{* * *}$ & $-0.79^{* * *}$ & $0.39^{*}$ & $0.39^{*}$ & $0.38^{*}$ & $-0.05^{\mathrm{ns}}$ & $0.26^{\mathrm{ns}}$ & $-0.20^{\mathrm{ns}}$ & $-0.53^{* *}$ & $0.83^{* * *}$ & $0.98^{* * *}$ & $0.87^{* * *}$ \\
\hline
\end{tabular}

Correlation analysis was performed using PROC CORR procedure (SAS 9.3, SAS Inst. 2002). Minus signs indicate negative correlations. ns, not significant. *, significant at $\mathrm{P}=0.05{ }^{* *}$, significant at $\mathrm{P}=0.01 .^{* * *}$, significant at $\mathrm{P}=0.001$. DPI, Days post inoculation with Verticillium longisporum. AUDPC, net area under disease progress curve, VL DNA, Verticillium longisporum DNA in hypocotyl. RS, relative stunting. PB, number of primary branches per plant. E, Transpiration rate. gs, stomatal conductance of $\mathrm{CO}_{2}$. A, photosynthesis rate. WUE, water use efficiency. LPC, leaf proline content. HPC, hHypocotyl proline content. RWC, leaf relative water content. PH, pPlant height. SDM, shoot dry matter. RDM, root dry matter. TDM, total dry matter. 
Appendix 4.10 Coefficients of determination (b), partial regression coefficients $\left(r^{2}\right)$, and $p$-values of stepwise regression analysis indicating the effects of genotype, Verticillium longisporum infection and drought stress on disease, physiological and agronomic traits of two $B$. napus genotypes at $28 \mathrm{DPI}$.

\begin{tabular}{|c|c|c|c|c|c|c|c|}
\hline \multirow{2}{*}{\multicolumn{2}{|c|}{$\begin{array}{l}\text { Factors } \\
\text { Parameters' }\end{array}$}} & \multicolumn{2}{|c|}{ Genotype } & \multicolumn{2}{|c|}{ V. longisporum } & \multicolumn{2}{|c|}{ Drought } \\
\hline & & $b$ & Partial $R^{2}$ & $b$ & Partial $R^{2}$ & $b$ & Partial $\mathrm{R}^{2}$ \\
\hline \multirow{4}{*}{ 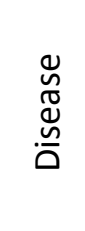 } & AUDPC & 9.3 & $0.11^{* * *}$ & 24.6 & $0.76^{* * *}$ & ns & ns \\
\hline & VL DNA & 331.7 & $0.29^{* * *}$ & 368.9 & $0.35^{* *}$ & ns & ns \\
\hline & RS & 13.7 & $0.05^{* *}$ & 57.5 & $0.87^{* * *}$ & ns & ns \\
\hline & PB & ns & ns & 3.0 & $0.77^{* * *}$ & ns & ns \\
\hline \multirow{7}{*}{$\begin{array}{l}\overline{0} \\
\frac{0}{00} \\
\frac{0}{0} \\
\frac{0}{n} \\
\frac{c}{a}\end{array}$} & $E$ & ns & ns & ns & ns & -1.7 & $0.89^{* * *}$ \\
\hline & gs & ns & ns & ns & ns & -0.2 & $0.88^{* * *}$ \\
\hline & $A$ & ns & ns & ns & ns & -10.3 & $0.93^{* * *}$ \\
\hline & WUE & ns & ns & ns & ns & 7.1 & $0.41^{* * *}$ \\
\hline & RWC & ns & ns & ns & ns & -7.2 & $0.66^{* * *}$ \\
\hline & LPC & 3.8 & $0.01^{*}$ & ns & ns & 41.0 & $0.97^{* * *}$ \\
\hline & HPC & 7.3 & $0.08^{*}$ & 7.7 & $0.09^{*}$ & 20.1 & $0.58^{* * *}$ \\
\hline \multirow{4}{*}{ 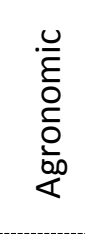 } & $\mathrm{PH}$ & ns & ns & -32.7 & $0.61^{* * *}$ & ns & ns \\
\hline & SDM & ns & ns & -6.0 & $0.73^{* * *}$ & ns & ns \\
\hline & RDM & -1.2 & $0.23^{* *}$ & -1.6 & $0.39^{* *}$ & -0.9 & $0.12^{* *}$ \\
\hline & TDM & -1.9 & $0.05^{*}$ & -7.5 & $0.70^{* * *}$ & -2.2 & $0.06^{*}$ \\
\hline
\end{tabular}

Mean data obtained from fully irrigated and severely stressed treatments of two independent experiments were used. Drought treatments (30\% FC) were applied for four weeks starting from 7 days after inoculation with Verticillium longisporum. AUDPC, net area under disease progress curve. VL DNA, Verticillium longisporum DNA in hypocotyl. RS, relative stunting. PB, number of primary branches per plant. E, transpiration rate. gs, stomatal conductance of $\mathrm{CO}_{2}$. A, photosynthesis rate. WUE, water use efficiency. RWC, leaf relative water content. LPC, leaf proline content. HPC, hypocotyl proline content. PH, plant height. SDM, shoot dry matter. RDM, root dry matter. TDM, total dry matter. ns, not significant. ${ }^{*}$, significant at $\mathrm{P} \leq 0.05 .{ }^{* *}$. significant at $\mathrm{P} \leq 0.01$. ${ }^{* * *}$, significant at $\mathrm{P} \leq 0.001$. 
Appendix 4.11 Sequential path model indicating stepwise regression coefficients of determination (b), partial regression coefficients $\left(r^{2}\right)$, Pearson's correlation coefficients $(r)$ and probability values of variables predicting total dry matter yield in Brassica napus genotypes subjected to drought stress and Verticillium longisporum infection at $28 \mathrm{DPI}$.

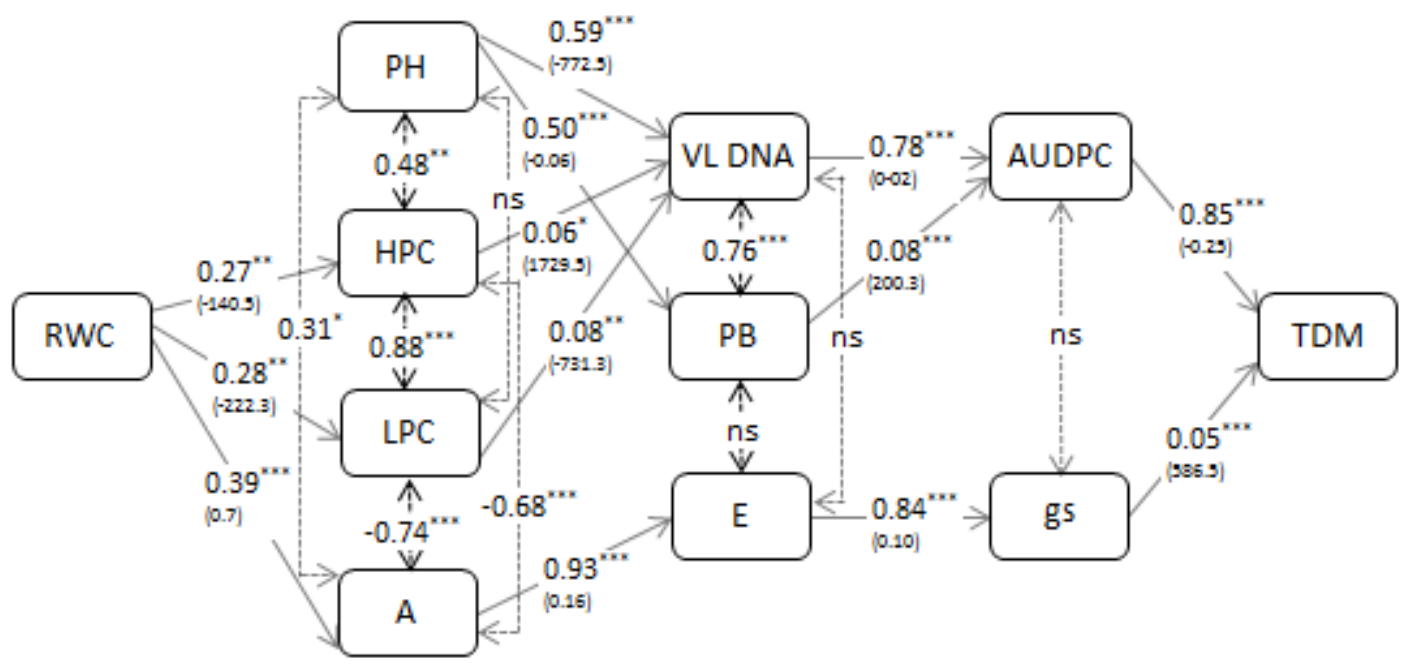

Mean data obtained from 30 plants of two independent experiments were used for analysis. Drought treatments were applied for four weeks starting from 7 days after inoculation with Verticillium longisporum. Solid arrows show stepwise regression analysis with Partial regression coefficients. Coefficients of determination are indicated in brackets. Dotted arrows show coefficients of Pearson's correlation indicating relationships within the predicting variables. AUDPC, net area under disease progress curve. VL DNA, Verticillium longisporum DNA in hypocotyl. PB, number of primary branches per plant. $\mathbf{E}$, transpiration rate. gs, stomatal conductance of $\mathrm{CO}_{2}$. $\mathbf{A}$, photosynthesis rate. $\mathbf{R W C}$, leaf relative water content. LPC, leaf proline content. HPC, hypocotyl proline content. PH, plant height. TDM, total dry matter. ns, not significant. ${ }^{*}$, significant at $\mathrm{P}=0.05$. $* *$, significant at $\mathrm{P}=0.01$. $* * *$, significant at $P=0.001$. 
Appendix 4.12 Principal component analysis of 16 diseases, physiological and agronomic variables measured from two Brassica napus genotypes subjected to drought stress and infection with Verticillium longisporum at $49 \mathrm{DPI}$.

\begin{tabular}{ccc} 
Variables & PC1 & PC2 \\
\hline AUDPC & 0,251 & $-0,297$ \\
VL DNA & 0,256 & $-0,251$ \\
PB & 0,215 & $-0,252$ \\
RS & 0,248 & $-0,295$ \\
E & $-0,221$ & $-0,287$ \\
gs & $-0,225$ & $-0,250$ \\
A & $-0,209$ & $-0,296$ \\
WUE & 0,169 & 0,295 \\
RWC & $-0,159$ & $-0,304$ \\
LPC & 0,176 & 0,336 \\
HPC & 0,144 & 0,345 \\
PH & $-0,310$ & 0,178 \\
HD & $-0,310$ & $-0,019$ \\
SDM & $-0,332$ & 0,037 \\
RDM & $-0,317$ & 0,129 \\
TDM & $-0,335$ & 0,061 \\
\hline Variance explained & $51 \%$ & $32 \%$
\end{tabular}

Only the first two principal components that explained much of the total variation are shown. Parameters included in the PCA: AUDPC, net area under disease progress curve. VL DNA, Verticillium longisporum DNA in hypocotyl. PB, number of primary branches per plant. RS, relative stunting. E, transpiration rate. gs, stomatal conductance of $\mathrm{CO}_{2}$. A, photosynthesis rate. RWC, leaf relative water content. LPC, leaf proline content. HPC, hypocotyl proline content. PH, plant height. HD, hypocotyl diameter. SDM, shoot dry matter. RDM, root dry matter. TDM, total dry matter. 


\section{Appendix 4.13 Phenological growth stages and $\mathrm{BBCH}$-identification keys of oilseed rape}

Oilseed rape Weber and Bleiholder, 1900; Lancashire et al., 1991

Phenological growth stages and $\mathrm{BBCH}$-identitication keys ot oilseed rape

(Brassica napus L. ssp. napus)

\begin{tabular}{ll}
\hline Code & Description \\
\hline Principal growth stage 0: Germination \\
00 & Dry seed \\
01 & Beginning of seed imbibition \\
03 & Seed imbibition complete \\
05 & Radicle emerged from seed \\
07 & Hypocotyl with cotyledons emerged from seed \\
08 & Hypocotyl with cotyledons growing towards soil surface \\
09 & Emergence: cotyledons emerge through soll surface \\
\hline Principal growth stage 1: Leat development' \\
10 & Cotyledons completely unfolded \\
11 & First leaf unfolded \\
12 & 2 leaves unfolded \\
13 & 3 leaves unfolded \\
1 & Stages continuous till... \\
19 & 9 or more leaves unfolded \\
\hline
\end{tabular}

Principal growth stage 2: Formation of side shoots

\begin{tabular}{|c|c|}
\hline $\begin{array}{l}20 \\
21 \\
22\end{array}$ & $\begin{array}{l}\text { No side shoots } \\
\text { Beginning of side shoot development first side shoot detectable } \\
2 \text { side shoots detectable } \\
3 \text { side shoots detectable } \\
\text { Stages continuous till ... } \\
\text { End of side shoot development: } 9 \text { or more side shoots } \\
\text { detectable }\end{array}$ \\
\hline \multicolumn{2}{|c|}{ Principal growth stage 3: Stem elongation ${ }^{x}$} \\
\hline & $\begin{array}{l}\text { Beginning of stem elongation: no internodes ("roselte") } \\
1 \text { visibly extended internode } \\
2 \text { visibly extended internodes } \\
3 \text { visibly extended internodes } \\
\text { Stages continuous till .... } \\
9 \text { or more vis ibly extended internodes }\end{array}$ \\
\hline
\end{tabular}

Stem elongation may occur earller than stage stage 19 ; in this case continue with stage 20

Visibly extended Internode $n$ develops between leat $n$ and leaf $n+1$

\begin{tabular}{ll} 
Principal growth stage 5: Intlorescence emergence \\
50 & Flower buds present, still enclosed by leaves \\
51 & Flower buds visible from above ("green bud") \\
52 & Flower buds free, level with the youngest leaves \\
53 & Flower buds raised above the youngest leaves \\
55 & Individual flower buds (main inflorescence) visible but still dosed \\
57 & Individual flower buds (secondary inflorescences) visible but stl \\
59 & closed \\
\hline
\end{tabular}

Principal growth stage 6: Flowering

$60 \quad$ First flovers open

$6110 \%$ of flowers on main raceme open, main raceme elongating

$62 \quad 20 \%$ of flowers on main raceme open

$63 \quad 30 \%$ of flowers on main raceme open

$64 \quad 40 \%$ of flowers on main raceme open

65 Full flowering: $50 \%$ flowers on main raceme open,

older petals falling

67 Flowering declining: majority of petals fallen

69 End of flowering

Principal growth stage 7: Development of truit

$71 \quad 10 \%$ of pods have reached final size

$72 \quad 20 \%$ of pods have reached final size

$73 \quad 30 \%$ of pods have reached final size $74 \quad 40 \%$ of pods have reached final size $50 \%$ of pods have reached final size $60 \%$ of pods have reached final size $70 \%$ of pods have reached final size $80 \%$ of pods have reached final size Nearly all pods have reached final size

Principal growth stage 8: Ripening

\begin{tabular}{ll}
80 & Beginning of ripening: seed green, filling pod cavity \\
81 & $10 \%$ of pods ripe, seeds dark and hard \\
82 & $20 \%$ of pods ripe, seeds dark and hard \\
83 & $30 \%$ of pods ripe, seeds dark and hard \\
84 & $40 \%$ of pods ripe, seeds dark and hard \\
85 & $50 \%$ of pods ripe, seeds dark and hard \\
86 & $60 \%$ of pods ripe, seeds dark and hard \\
87 & $70 \%$ of pods ripe, seeds dark and hard \\
88 & $80 \%$ of pods ripe, seeds dark and hard \\
89 & Fully ripe: nearly all pods ripe, seeds dark and hard \\
\hline
\end{tabular}

Principal growth stage 9: Senescence

$97 \quad$ Plant dead and dry

$99 \quad$ Harvested product 


\section{Acknowledgements}

First and foremost, I would like to thank God for giving me the wisdom, strength and endurance to complete my study.

I would like to extend my sincere gratitude to my supervisor Prof. Dr. Andreas von Tiedemann first of all for accepting me as his student then for his supervision, guidance and advice throughout my study period. He was always keen to give valuable and brilliant ideas which enriched my study. I learned a lot from his extraordinary experiences and expertise in phytopathology and skills in leadership. Thank you for your availability without any preconditions, for your precious time to go throughout my thesis and the critical comments and suggestions. My great gratitude goes to Prof. Dr. Petr Karlovsky for supervising my study and for his advice and support particularly with regard to the molecular studies. I am highly indebted to Prof. Dr. Heiko C. Becker for his absolute willingness to be my examiner.

My special thanks goes to Dr. Birger Koopmann for his unreserved guidance and advice from the first date of my study until the end. I really appreciate your multipurpose role in the group as a whole. Thank you for your guidance during my work on molecular topics and for your valuable comments and suggestions on my thesis in particular. Dr. Jessica Knüfer, Dr. Mark Winter and Dr. Magdalena Siebold are gratefully acknowledged for sharing me their skills and expertise and for those interesting scientific and social discussions. I would like to thank Dr. Anke Sirrenberg for her interest to read part of my thesis and her comments.

Jutta shapper is the first person to be sincerely acknowledged for all her assistance in the field trials and greenhouse studies. I also acknowledge the significant contribution of Hubertus Reintke, Evelin Vorbeck and Dagmar Tacke in the field and lab experiments. Thank you all for your help in the last years. Without your support, my work would not have been successful.

Many thanks go to the project partners at the University of Giessen, Department of Plant Breeding. Particularly Dr. Christian Obermeier, Prof. Dr. Wolfgang Friedt and Prof. Dr. Rod Snowdon are sincerely acknowledged for their unreserved collaboration which led to the successful completion of the project. I also thank all the breeding companies in the GFP for providing plant materials and for the fruitful cooperation. Special thanks go to Dr. Detlef Hauska, Dr. Maria Kaliff, Dr. Tobias Jesske, Dr. Werner Horn, Dr. Reinhard Hemker, Dr. Andreas Girke, Dr. Annika Spies, Dr. Peter Duchscherer, Ms Simone Sendke, Dr. Andreas Gertz, Dr. Hanna Meier zu Beerentrup. My sincere gratitude also goes to Mr. Stefan Lütke Entrup for the productive cooperation and organization of the fruitful annual GFP meetings. I am deeply 
grateful to Christine Kettner and Prof. Dr. Andrea Polle (Institute for Forest Botany, Forest Botany and Tree Physiology Section, Göttingen University) for their cooperation and technical help during my drought experiments.

To all staff members of the Division of General Plant Pathology and Crop Protection, Göttingen University, I would like to say thank you for the enjoyable social events and for providing me with a nice research facilities and condusive working environment. In particular, my special thanks go to Ms Martina Bode for the efficient facilitation of the academic and project related issues which substantially helped me to smoothly accomplish my study. Collective and individual acknowledgments are also owed to all my PhD colleagues; Christian Comberg, Geoffrey Onaga, Hendrik Hanekamp, Ines Eikenberg, Kerstin Höch, Lucia Ramos-Romero, Sandra Gerth, Tingting Wei and Xia Ha. Thank you guys for the nice time we had during the social events and our Tuesday seminar which greatly helped us to improve our work. It was really great to know you all and work with you.

I want to take this opportunity to thank my Ethiopian friends and their families here in Göttingen. Dr. Zerihum Teklemariam, Wondimeneh Taye, Denabo Billo and Dr. Yabibal Mulualem your presence was helpful and memorable. My special thanks goes to Cathrin Horstmann for her help in several administrative issues, for her constant encouragement and for the nice social events.

Last but not least, I extend my gratefulness to my brothers and sisters who contributed a lot for my today's position and for their everlasting love and faith. My Special thanks go to Gash Girma Gebray and his family who were my motivation since the beginning of my earliest studies. Emebet, Techane, Belayneh, Alganeh, Yimer, Yoseph, Zemede, Wondwesen and Shewangizaw, you have a special place in my life in particular and in Lopisso's family as a whole. Without your support, I would have not been reached to today's situation. Thank you Kokobe Gizaw for your continues support, advise, encouragement, and pray that helped me a lot for the successful completion of my study. May God bless you all!

This study was financed by GFP (an association for the promotion of private plant breeding in Germany), BMELV (German Federal Ministry for Food, Agriculture and Consumer Protection) and FNR (German Federal Agency of renewable resources) to which I am highly indebted. 


\section{Selected contributions in non-reviewed journal paper or conference proceedings}

Pfordt A., Zheng X., Eseola A. B., Wilch A., Koopmann B., Lopisso D. T., von Tiedemann A. 2016. Time course of infection, seed transmission and damage of Verticillium longisporum in oilseed rape. In: Berne S. and Javornik B. (Eds).12 ${ }^{\text {th }}$ International Verticillium Symposium. Ljubljana, Slovenia. Book of Abstracts, P.28.

Lopisso D. T., Eseola A. B., Koopmann B., and von Tiedemann A. 2016. The role of seed transmission of Verticillium longisporum in oilseed rape. The $60^{\text {th }}$ German Plant Protection Conference. Haale, Germany.

Alnajar D., Lopisso D. T., Koopmann B., and von Tiedemann A. 2016. Potential of root associated fungal and bacterial biocontrol agents (BCAs) against Verticillium longisporum infection of oilseed rape. The $60^{\text {th }}$ German Plant Protection Conference. Haale, Germany.

von Tiedemann A, Eynck C., Riediger N., Lopisso D. T., Kamble A., Knüfer J., Novakazi F., Zheng X., Siebold M., Koopmann B., 2015. A hidden pathogen with uncommon properties - reviewing the state-of-the-art of Verticillium longisporum on oilseed rape. $14^{\text {th }}$ International Rapeseed Congress, Book of Abstracts, p.115.

Hossain M. A., Lopisso D. T., Snowdon R., von Tiedemann A., Friedt W., Obermeier C. 2015. Mining QTI for candidate genes involved in resistance of oilseed rape against Verticillium longisporum by an integrative omics approach. $14^{\text {th }}$ International Rapeseed Congress, Book of Abstracts, p.365.

Lopisso D. T., Knüfer J., Koopmann B., von Tiedemann A. 2015. Effects of host-resistance to Verticillium longisporum on the performance of winter oilseed rape (Brassica napus) under drought stress. XVIII. International Plant Protection Congress, Berlin 2015. Book of Abstracts, p.540.

Knüfer J. Lopisso D. T., Koopmann B., von Tiedemann A. 2014. Classification of winter oilseed rape resistance towards the soil-borne pathogen Verticillium longisporum by quantitative PCR. 59 . Deutsche Pflanzenschutztagung, Freiburg, Germany, Kurzfassungen der Beiträge, Julius-KühnArchiv 447, S.354.

Lopisso D. T., Knüfer J., Koopmann B. and von Tiedemann A. 2014. Impact of cultivar resistance to Verticillium longisporum on drought stress tolerance of winter oilseed rape (Brassica napus). 59. Deutsche Pflanzenschutztagung, Freiburg, Germany, Kurzfassungen der Beiträge, Julius-KühnArchiv 447, S.355.

Lopisso D. T., Knüfer K., Koopmann B, and von Tiedemann A. 2015. Phenotypinig of a 'hidden' pathogen - quantifying resistance in winter oilseed rape lines (Brassica napus) to Verticillium longisporum, PhenoDays 2014. Beaune, France.

Lopisso D. T., Knüfer J., Koopmann B: and von Tiedemann A. 2013. Winter oilseed rape physiology, gene 
expression and agronomic performance during drought stress and infection with Verticillium longisporum. In: Koopmann B., von Tiedemann A. (Hg.) $11^{\text {th }}$ International Verticillium Symposium. Braunschweig, Germany, Book of Abstracts, P.47.

Hossain M. A., Obermeier C., Snowdon R., Lopisso D. T., von Tiedemann A. and Friedt W. 2013. Association of phenylpropanoid metabolites with resistance of rapeseed to Verticillium longisporum. $11^{\text {th }}$ International Verticillium Symposium. Braunschweig, Germany, Book of Abstracts, P.100.

Lopisso D. T., Knüfer J., Koopmann B., von Tiedemann A. 2013. Response of oilseed rape (Brassic napus L.) to combined effects of drought stress and Verticillium longisporum infestation. In: Koopmann B, Cook S, Evans N, Ulber B (Hg.) Integrated Control in Oilseed Crops - IOBC-WPRS Bulletin VOL.96. IOBC-Verlag, Gent, Belgium, 213-214.

Lopisso D. T., Koopmann B., von Tiedemann A. 2012. Physiological and morphological responses in oilseed rape (B. napus) during drought stress and infection with Verticillium longisporum. 58. Deutsche Pflanzenschutztagung, 10.-14.September 2012, Braunschweig. Kurzfassungen der Beiträge, Julius-Kühn-Archiv 438, P.403.

\section{Master's project supervision}

Eseola A. B. The role of seed transmission of Verticillium longisporum in oilseed rape. Division of General Plant Pathology and Crop Protection, Department of Crop Sciences, Faculty of Agricultural Sciences, Georg-August-Universität Göttingen, Germany. November, 2016.

Alnajar D. Potential of root associated fungal and bacterial biocontrol agents against Verticillium longisporum in oilseed rape. Division of General Plant Pathology and Crop Protection, Department of Crop Sciences, Faculty of Agricultural Sciences, Georg-August-Universität Göttingen, Germany. Germany. July, 2016.

Kühlmann V. Plant growth promotion and disease control potential of soilborne fungal biocontrol agents on sugar beets infected with Verticillium wilt. Division of General Plant Pathology and Crop Protection, Department of Crop Sciences, Faculty of Agricultural Sciences, Georg-AugustUniversität Göttingen, Germany. Germany. December, 2015.

Farooq M. Differential responses of oilseed rape (Brassica napus L.) to infection with fungal and bacterial vascular pathogens. Division of General Plant Pathology and Crop Protection, Department of Crop Sciences, Faculty of Agricultural Sciences, Georg-August-Universität Göttingen, Germany. September, 2013. 


\section{CURRICULUM VITAE}

\section{PERSONAL DETAILS}

Full name:

Date of Birth:

Place of birth:

Nationality:
Daniel Teshome Lopisso

August 27, 1980

Addis Ababa, Ethiopia

Ethiopian

\section{EDUCATIONAL BACKGROUND}

1. PhD in Plant Pathology (2011-2014), graduated with highest honor (Summa Cum Laude) from Georg-August-Universität Göttingen, Germany

2. MSc in Plant Pathology and Entomology (2008 - 2010), graduated with distinction from Wageningen University, The Netherlands

3. BSc degree in Plant Production and Dry Land Farming (2003-2005), graduated with distinction from Debub University, Ethiopia

4. Diploma in Plant Science and Technology (1997-1999), graduated with distinction from Debub University, Ethiopia

\section{WORK EXPERIENCE}

1. Post Doctorate at Georg-August-Universität Göttingen, Germany since September 2014: Coordination and supervision (master's students and project staff) of a bilateral (GermanGreece) project on biological crop protection.

2. PhD student and Researcher at Georg-August-Universität Göttingen, Germany from April 2011 to August 2014: Conducted and supervised a trilateral project (plant pathology and crop protection) funded by the German private plant breeding association and several European seed companies.

3. Lecturer and Researcher at Jimma University, Ethiopia from 2005 to 2008: Teaching, research, student and staff supervision, community based training, etc.

4. Academic and Research Technical Assistant at Harramaya University, Ethiopia from 2002 to 2003: Assisted field and laboratory researches in the crop protection section of the department of plant sciences.

5. Research Technical Assistant at Awassa Agricultural Research Organization, Ethiopia from 2000 to 2001: Assisted plant breeding and crop protection field researches.

\section{HONORS AND AWARDS}

1. Outstanding international student: Netherlands Organization for International Cooperation in Higher Education (Nuffic), August,2008

2. Excellence in teaching: Jimma University College of Agriculture and Veterinary Medicine, Ethiopia, August 2007

3. Innovative and outstanding research and scientific presentation: Scientific Committee of the IOBC working group "Integrated Control in Oilseed Crops" October 2013, Luxemburg. 


\section{Declaration}

I, hereby, declare that this dissertation was undertaken independently and without any unaccredited aid.

Göttingen, September 2014 